\title{
The Amaryllididae of Australia (Crustacea: Amphipoda: Lysianassoidea)
}

\author{
J.K. LOWRY AND H.E. STODDART \\ Division of Invertebrate Zoology, \\ Australian Museum, 6 College Street, Sydney NSW 2010, Australia \\ jimlowry@crustacea.net \\ helenst@austmus.gov.au
}

\begin{abstract}
Lowry \& Stoddart (in press) established the lysianassoid amphipod family Amaryllididae. In the following paper the systematics of the diverse Australian amaryllidid fauna is presented. All amaryllidid genera are revised and rediagnosed and a key to genera is provided. Five genera and 23 species are recorded from Australian waters. Bathyamaryllis is recorded from Australia for the first time and new evidence indicates that Amaryllis is confined to Australia and possibly the New Zealand area. Two subfamilies (Amaryllidinae and Vijayiinae), three genera (Bamarooka, Devo and Wonga) and 20 species are new. The new species are Amaryllis carrascoi, A. croca, A. dianae, A. kamata, A. keablei, A. migo, A. moona, A. olinda, A. philatelica, A. quokka, A. spencerensis, Bamarooka anomala, B. dinjerra, B. endota, B. kimbla, B. tropicalis, Bathyamaryllis kapala, Devo dubuc, D. grahami and Wonga wonga. Distribution, depth and habitat notes are given for all species. Amaryllidids form two natural groups: a presumed free-living deep-water group with a subquadrate mouthpart bundle (Vijayiinae) and a presumed commensal shallow-water group with a subconical mouthpart bundle (Amaryllidinae). Except for Vijaya tenuipes the vijayiines are exclusively found in the deep seas of the North and South Atlantic and South Pacific Oceans. The amaryllidines are found in shallow temperate and tropical seas of the southern hemisphere. The largest diversity of genera and species is currently known from Australian waters, but the African and South American faunas have not been adequately described.
\end{abstract}

LOWRY, J.K., \& H.E. STODDART, 2002. The Amaryllididae of Australia (Crustacea: Amphipoda: Lysianassoidea). Records of the Australian Museum 54(2): 129-214.

The amaryllidids have a long taxonomic history. Haswell (1879: 253) originally described the genus Amaryllis (in the "sub-family Stegocephalides") for two species, $A$. macrophthalma and A. brevicornis, from southeastern Australia. Neither species was well described. Stebbing (1888) synonymised A. brevicornis with A. macrophthalma, transferred Amaryllis to the Lysianassidae and described two new species: A. bathycephala from southeastern Australia and $A$. haswelli from the North Atlantic.

Bonnier (1896) described A. pulchellus from the Bay of Biscay in the North Atlantic Ocean. Walker (1904) described
Vijaya tenuipes, a new genus and species of amaryllidid amphipod from Sri Lanka. He based the genus on the fact that the male had a callynophore on antenna 1. Stebbing (1910a) synonymised Vijaya with Amaryllis because males of that genus also have callynophores. However, Lowry \& Stoddart (in press) have redescribed Vijaya tenuipes and shown that Vijaya is a valid genus more closely related to Pseudamaryllis and Bathyamaryllis than to Amaryllis. Chevreux (1911) described $A$. rostrata from the deep sea of the North Atlantic Ocean and K.H. Barnard (1925) described A. conocephalus from southern Africa. 
While studying material from the Siboga Expedition, Pirlot (1933) realised that there were two groups within the accumulated species of Amaryllis. One group, Amaryllis, with no rostrum and a short peduncle on antenna 1 , included the shallow-water coastal species A. macrophthalma, A. bathycephala and $V$. tenuipes. The other group, Bathyamaryllis, with a prominent rostrum and a long peduncle on antenna 1 , included the deep-water species $B$. haswelli, $B$. perezii Pirlot, B. rostrata and $B$. conocephalus. This concept is reflected in our present recognition of two subfamilies in the Amaryllididae, but with Vijaya included in the otherwise exclusively deep-water group.

Andres (1981) described a fourth amaryllidid genus, Pseudamaryllis, from the abyssal zone in the Red Sea. Ledoyer (1986) reduced this genus to a subgenus of Amaryllis but Lowry \& Stoddart (1994) showed that Pseudamaryllis is a distinct genus related to Bathyamaryllis. Lowry \& Stoddart (1987) described a shallow-water genus, Erikus, from southern South America. Ren (1998) described a new genus, Paravijaya, from the South China Sea, but we do not consider this genus to be distinct from Pseudamaryllis.

There have been only a few subsequent reports of the described deep-water species (Chevreux, 1903; Chevreux, 1935; Stephensen, 1923; Griffiths, 1977; Ledoyer, 1986; Lawson et al., 1993); but shallow-water amaryllidids have been widely reported as Amaryllis macrophthalma (Fig. 1).

A major impediment to the development of amaryllidid systematics has been the persistent misidentification of $A$. macrophthalma from many areas. These erroneous reports (e.g., Stebbing, 1888; Thomson, 1902; Walker, 1909; Chilton, 1912; K.H. Barnard, 1916; Schellenberg, 1926, 1931; Griffiths, 1973-1977; Ledoyer, 1978-1986; Alonso, 1987; Lyons \& Myers, 1991) have hindered the recognition of generic and specific level diversity in the amaryllidine part of this group.

In this paper we describe two new subfamilies, Amaryllidinae and Vijayiinae, three new genera, Bamarooka, Devo and Wonga and 20 new species; we revise, diagnose and provide keys for all genera; we confine the genus Amaryllis to the Australasian area and the species A. macrophthalma to southeastern Australia.

\section{Materials and methods}

Material used in this study comes from a very large collection of Australian lysianassoid amphipods belonging to, or currently on loan to, the Australian Museum. Most of the inshore Australian coastal waters are represented plus continental shelf samples from southeastern and northwestern Australia and slope samples from off New South Wales and the Bass Strait. However, the abundance of samples and thoroughness of sampling is far from balanced. The southeastern coastline is well represented, but we have no samples at all from, for example, the Gulf of Carpentaria.

Major collections include: CSIRO, North West Shelf survey (CSIRO NWS); Victorian Ministry for Conservation, Marine Studies Group, Western Port Bay Environmental Study (WPBES) and Port Phillip Bay Environmental Study (PPBES); Museum of Victoria, Bass Strait Survey (BSS), SLOPE survey, and expeditions to southern and southwestern Australia, South Australia and Tasmania; New South Wales Department of Agriculture, Division of
Fisheries, dredge samples taken by the FRV Kapala on the New South Wales continental shelf and slope; Australian Museum expeditions to Cobourg Peninsula, Northern Territory, Western Australia, Kangaroo Island, South Australia, Lizard Island, Queensland, and the New South Wales coast.

Material is deposited in: Australian Museum, Sydney (AM); Museum Victoria, Melbourne, [formerly the National Museum of Victoria] (NMV); South Australian Museum, Adelaide (SAMA); and Western Australian Museum (WAM).

The taxonomic descriptions presented in this paper were generated from a DELTA (Dallwitz et al., 1993; Dallwitz et al., 1998) database of world amaryllidid amphipod species. Italicised phrases in the descriptions indicate diagnostic characters.

Descriptions of setae on the mandibular palp follow the scheme of Lowry \& Stoddart (1993). Descriptions of gnathopod palmar angles follow the scheme of Poore \& Lowry (1997). Measurements of whole animals are made from the front of the head (including rostrum) to the base of the telson.

Abbreviations used on figures in the text are: A, antenna; C, coxa; E, epistome; EP, epimeron; G, gnathopod; H, head; LL, lower lip; MD, mandible; MDp, mandibular palp; MP, maxilliped; MPIP, maxilliped inner plate; MPOP, maxilliped outer plate; MPp, maxilliped palp; MX, maxilla; MX1IP, maxilla 1 inner plate; MX1OP, maxilla 1 outer plate; P, pereopod; T, telson; $\mathrm{U}$, uropod; UR, urosome; 1, left; r, right.

\section{Systematics}

\section{Variation, development and sexual dimorphism}

Descriptions of species are, wherever possible, based on mature females with dimorphic characters based on mature males. However, within species morphological variation results from age/size and sexual maturity.

Variation resulting from age/size occurs in characters such as: the number of articles in the accessory flagellum of antenna 1 and the flagella of antennae 1 and 2; the number of setae on the propodus of gnathopod 1; the number of robust setae on the palm of gnathopod 2; setation of the distal articles of the pereopods; number of robust setae on the uropods. This variation is much greater in species that grow to a large size (for example A. macrophthalma) than in species that are sexually mature at a smaller size (for example A. moona).

Many characters differ between mature males and mature females. Some of these differences are because mature males are usually smaller than mature females of the same species. Sexual dimorphism can be expressed in mature males in the following characters: eye slightly enlarged; well-developed callynophore, or in species where a callynophore is present in females, then it is more strongly developed in the mature male; calceoli on antennae 1 and 2 or only on antenna 2; elongation of antenna 2; development of brush setae on peduncular articles 4 and 5 of antenna 2 ; development of strong setae on the distal half of mandibular palp article 2 and an increase in the number of D-setae on article 3; development of a fringe of setae on the posterior margin of the merus and carpus of pereopods 3 and 4 ; development of plumose setae on one or both rami of uropod 3 .

Not all character states are expressed in all, or even one genus. 


\section{Amaryllididae Lowry \& Stoddart}

Amaryllididae Lowry \& Stoddart, 2002: 165.

Diagnosis. Head much deeper than long, with lateral cephalic lobe weak, midanterior notch present or absent. Epistome and upper lip fused. Mandible, left lacinia mobilis a stemmed serrate blade; accessory setal row well developed, usually with intermediate setae, with a large tuft of setae distally; molar a slightly setose flap with setose margins. Maxilla 1, outer plate with setal-teeth in a $6 / 5$ arrangement, palp absent. Maxilliped, outer plate without distal or medial slender or robust setae; palp well developed, article 4 reduced. Gnathopod 1 simple, coxa vestigial, merus and carpus not rotated, posterior margin of propodus serrate, dactylus simple, without subterminal tooth. Gnathopod 2, posterodistal corner of propodus with medial and lateral robust setae. Pereopods 3-7 simple; propodus without posterodistal spur. Telson with slight to medium cleft.

Type genus. Amaryllis Haswell, 1879.

Generic composition. The family contains eight genera in two subfamilies: Amaryllidinae n.subfam. with Amaryllis Haswell, 1879; Bamarooka n.gen.; Erikus Lowry \& Stoddart, 1987 and Wonga n.gen.; and Vijayiinae n.subfam. with Bathyamaryllis Pirlot, 1933; Devo n.gen.; Pseudamaryllis Andres, 1981 and Vijaya Walker, 1904.

Remarks. Amaryllidids are very distinctive amphipods. They are distinguished from other lysianassoid family groups by their head shape; maxilla 1 without a palp; extremely reduced gnathopod 1 coxa; and gnathopod 1 propodus with serrate posterior margin. Most are immediately recognizable by their very deep head shape with midanterior notch.

\section{Key to subfamilies and genera of the Amaryllididae}

1 Mouthpart bundle subquadrate. Gnathopod 1, posterior margin of propodus with robust setae. Epimeron 3 with notch immediately above acute posteroventral corner (Vijayiinae)

_ Mouthpart bundle conical or subconical. Gnathopod 1, posterior margin of propodus without robust setae. Epimeron 3 without notch or with notch slightly or well above posteroventral corner (Amaryllidinae)

2 Antenna 1 peduncular article 1 not ball-shaped proximally, article 2 medium length (about as long as broad). Uropod 3 outer ramus 1-articulate

— Antenna 1 peduncular article 1 ball-shaped proximally, article 2 long (length more than $1.5 \times$ length). Uropod 3 outer ramus 2 articulate

3 Head with or without rostrum. Eye reniform. Antenna 1, callynophore present in female. Antenna 2 not elongate in male

- Head with rostrum. Eye ventrally tapered. Antenna 1, callynophore absent in female. Antenna 2 elongate in male

4 Rostrum cone-shaped. Pereopods 5 to 7, dactyls long, slender. Pereopod 7 basis rounded posteriorly, posteroventral corner rounded

- Rostrum anteriorly rounded or truncated, not cone-shaped. Pereopods 5 to 7 , dactyls short, slender. Pereopod 7 basis subrectangular, posteroventral corner subquadrate

5 Pereopod 4 coxa, anterior and posterior margins subparallel 6

— Pereopod 4 coxa, anterior margin slightly obtuse, posterior margin rounded Bamarooka

6 Mouthpart bundle subconical. Pereopods 5-7, distal articles elongate, slender. Uropod 3 rami lanceolate

Mouthpart bundle conical. Pereopods 5-7, distal articles short, broad. Uropod 3 rami clasper-like Wonga

$7 \quad$ Mandibular palp article 3 with proximal A3-seta. Pereopods 3 and 4 in male with fringe of plumose setae on posterior margin of merus and carpus. Uropod 3 outer ramus 2-articulate Erikus Mandibular palp article 3 without proximal A3-seta. Pereopods 3 and 4 in male without fringe of setae on posterior margin of merus and carpus. Uropod 3 outer ramus 1-articulate 


\section{Amaryllidinae n.subfam.}

Diagnosis. Mouthpart bundle subconical or conical. Gnathopod 1 propodus without robust setae along posterior margin. Epimeron 3 without a notch or with notch slightly or well above posteroventral corner.

Type genus. Amaryllis Haswell, 1879.

Generic composition. The subfamily contains four genera: Amaryllis Haswell, 1879; Bamarooka n.gen.; Erikus Lowry \& Stoddart, 1987; and Wonga n.gen.

Remarks. The amaryllidines are found in shallow temperate and tropical seas of the southern hemisphere. The largest diversity of genera and species is currently known from Australian waters, but the African and South American faunas have not been adequately described.

\section{Amaryllis Haswell}

Amaryllis Haswell, 1879: 253.-Haswell, 1880: 31.-Haswell, 1882: 227.-Stebbing, 1888: 698.-Della Valle, 1893: 781.-Stebbing, 1906: 23.-Pirlot, 1933: 122 (in part, part = Vijaya Walker, 1904).-J.L. Barnard, 1969: 320.-Ledoyer, 1986: 717 (in part, part = Pseudamaryllis). - Barnard \& Karaman, 1991: 460.

Diagnosis. Mouthpart bundle subconical. Mandible palp article 3 without A3-seta. Pereopod 4 coxa with anterior and posterior margins subparallel. Pereopods 5-7 with distal articles elongate. Uropod 3 rami lanceolate; without plumose setae in male and female; outer ramus 1-articulate.

Type species. Amaryllis macrophthalma Haswell, 1879, selected by Pirlot, 1933.

Species composition. Amaryllis contains 13 species: A. brevicornis Haswell, 1879; A. carrascoi n.sp.; A. croca n.sp.; A. dianae n.sp.; A. kamata n.sp.; A. keablei n.sp.; A. macrophthalma Haswell, 1879; A. migo n.sp.; A. moona n.sp.; A. olinda n.sp.; A. quokka n.sp.; A. philatelica n.sp. and $A$. spencerensis n.sp.

Remarks. The genus Amaryllis (almost exclusively as A. macrophthalma) has been recorded from a wide geograph-

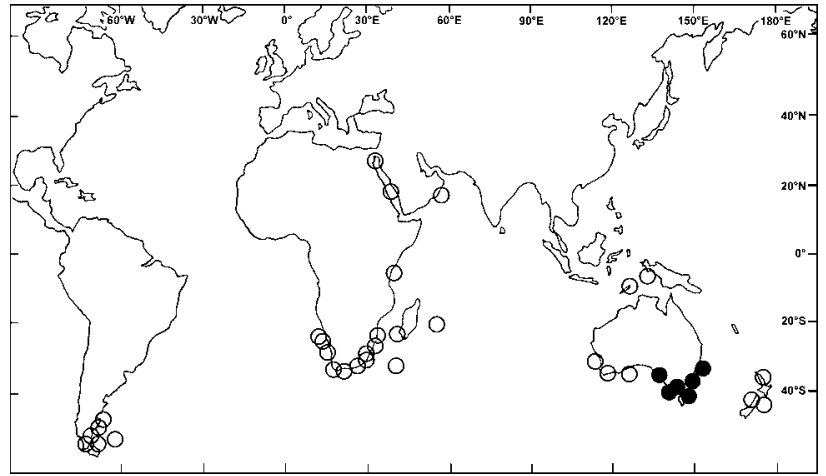

Figure 1. $\bigcirc$ literature records of "Amaryllis macrophthalma"; - distribution of Amaryllis macrophthalma.

ical area: from southeastern South America, through southern and eastern Africa, the Red Sea, Indonesia, New Zealand and all of southern Australia (see Fig. 1). Where the material has been illustrated or described we can, with our current knowledge of the Australian species of Amaryllis, exclude the non-Australasian records from $A$. macrophthalma. We are also convinced they do not belong to the genus Amaryllis (see Remarks for Erikus).

Amaryllis apparently occurs only in the Australasian area. Within Australia, it has not been recorded north of $30^{\circ} \mathrm{S}$ on either the east or west coast (Fig. 2).

Distribution. Currently confirmed only from Australia.

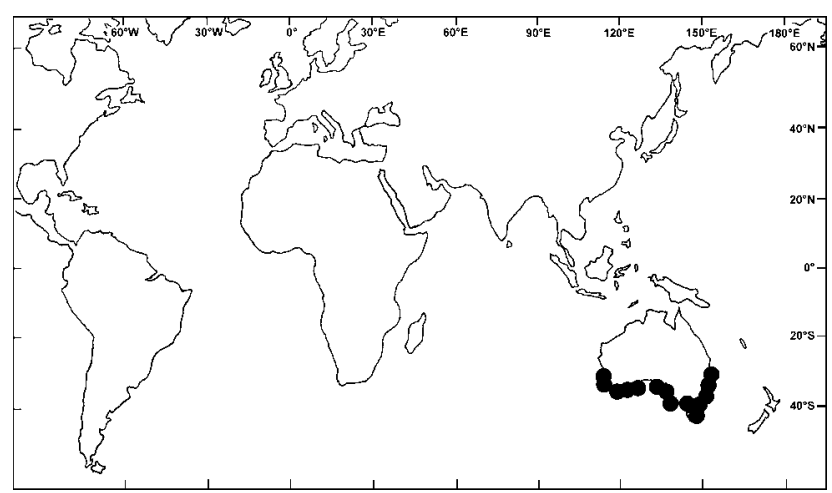

Figure 2. Distribution of the genus Amaryllis.

\section{Key to species of Amaryllis}

1 Epimeron 3 posterior margin with a single notch above posteroventral corner

Epimeron 3 posterior margin with more than a single notch .................................................. 2

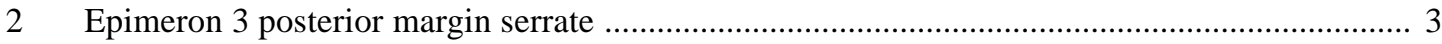

_ Epimeron 3 posterior margin with a notch plus small or tiny serrations above rounded posteroventral corner

3 Antenna 1 peduncular article 1 distal margin with well-developed medial spine. Epistome in lateral view with a mid-anterior hook. Gnathopod 2 of adult female with 1 lateral and 3-4 medial robust setae defining palm

Antenna 1 peduncular article 1 distal margin with small medial spine. Epistome in lateral view with a strong mid-anterior angle. Gnathopod 2 of adult female with 3-4 lateral and 4 medial robust setae defining palm 
4 Antenna 1 peduncular article 1 distal margin with small medial spine; flagellum without callynophore in female. Pereopod 7 basis subrectangular. Uropod 1 outer ramus without cuticular spikes between robust setae

Antenna 1 peduncular article 1 distal margin without medial spine; flagellum with weak callynophore in female. Pereopod 7 basis posteriorly rounded. Uropod 1 outer ramus with cuticular spikes between robust setae

5 Epistome in lateral view slightly angled. Gnathopod 2 of adult female with 3 lateral and 3 medial robust setae defining palm. Pereopod 7 basis posteroventral corner notched

A. carrascoi

Epistome in lateral view with slight depression above moderate angle. Gnathopod 2 of adult female with no lateral and 2-3 medial robust setae defining palm. Pereopod 7 basis posteroventral corner subquadrate

A. dianae

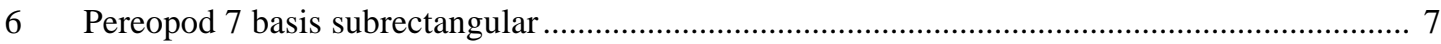

Pereopod 7 basis rounded posteriorly ................................................................................ 9

$7 \quad$ Antenna 1 peduncular article 1 distal margin with well-developed medial spine. Epimeron 3 posterior margin with notch well above rounded posteroventral corner.

_ Antenna 1 peduncular article 1 distal margin without medial spine. Epimeron 3 posterior margin with notch slightly above posteroventral corner

A. philatelica

8 Pereopod 7 basis posteroventral corner with a distinct notch. Gnathopod 2 palm of female with 2 lateral and 1 medial robust setae

Pereopod 7 basis posteroventral corner right-angled, without a notch. Gnathopod 2 palm of female with 2-4 lateral and 2-3 medial robust setae

A. macrophthalma

9 Left lacinia mobilis forming a broad cup distally, teeth reduced to irregular projections. Gnathopod 2 palm of female without lateral robust setae

_ Left lacinia mobilis forming a flat blade with several strong distal teeth. Gnathopod 2 palm of female with at least 1 lateral robust seta

10 Epistome almost straight in lateral view. Mandible accessory setal row without intermediate setae. Maxilliped outer plate with distomedial margin serrate. Gnathopod 2 palm slightly acute

_ Epistome with a broad midanterior bulge. Mandible accessory setal row with intermediate setae. Maxilliped outer plate with distomedial margin smooth. Gnathopod 2 palm transverse

11 Antenna 1 peduncular article 1 distal margin with small medial spine. Pereopod 7 basis posteroventral margin straight

Antenna 1 peduncular article 1 distal margin without medial spine.

Pereopod 7 basis posteroventral margin curved

A. olinda

12 Epistome in lateral view with small depression above moderate mid-anterior angle. Gnathopod 1 carpus subequal to propodus

A. kamata

Epistome in lateral view almost straight. Gnathopod 1 carpus shorter than propodus 


\section{Amaryllis brevicornis Haswell}

$$
\text { Figs. 3, } 4
$$

Amaryllis brevicornis Haswell, 1879: 254.-Haswell, 1882: 228.Whitelegge, 1889: 54.-J.L. Barnard, 1974: 140.-Springthorpe \& Lowry, 1994: 11.

Amaryllis sp. 3.-Hutchings et al., 1989: 362.

Type material. LECTOTYPE, female, $11.2 \mathrm{~mm}$, ovigerous (40 eggs), AM G5417; 3 PARALECTOTYPES, female, AM P37181; Port Jackson, New South Wales, Australia, [approx. $\left.33^{\circ} 51^{\prime} \mathrm{S} 151^{\circ} 16^{\prime} \mathrm{E}\right]$.

Springthorpe \& Lowry (1994:5) have discussed the general problem of Haswell "types". For Amaryllis brevicornis, two lots of material in the Australian Museum collection (AM G5417 and P3440) were regarded as syntypes. The first lot (G5417) was registered into the collection and labelled as "Type" by A.R. McCulloch in 1905; the material is from Port Jackson (the type locality and the only locality recorded for this species in Haswell's original publication of 1879). The second lot (P3440), was registered into the collection in 1912 and was not then regarded as type material. It is also from Port Jackson. However, the register entries for P3439 and P3441 are also A. brevicornis, from Griffiths Point and Port Stephens. This set of localities-Port Jackson, Port Stephens and Griffiths Point-are the three localities given by Haswell (1882) in his subsequent report of A. brevicornis material. It seems to us that the material of AM P3440 should not be regarded as type material.

The syntypes of A. brevicornis were mounted with glue onto a glass plate and stored in alcohol for more than 100 years. The specimens were heavily coated with precipitate from the preserving fluid. We have removed one specimen from the glass plate, peeled off the glue, and removed as much as possible of the accumulated deposit. However, many setae (for which insertion points can still be recognized) have been lost and some parts are still not clearly visible. Although we are designating this specimen as a lectotype, we have chosen to describe and illustrate in detail a female not from the type collection. The specimen described (from Clovelly Pool, AM P37116, just south of Port Jackson) agrees extremely well with all characters that can be determined from the lectotype.

One specimen from the original syntype series of 5 specimens is not A. brevicornis. It has a serrate epimeron 3 and is a specimen of $A$. keablei. It is now registered as AM P37180.

Additional material. New South WALES: 16 specimens, AM P37217, Sugarloaf Point, $32^{\circ} 26^{\prime} \mathrm{S} 152^{\circ} 32^{\prime} \mathrm{E}, 1-2 \mathrm{~m}$, coralline algae in rock pool, J.K. Lowry, 14 January 1981, stn NSW-135. 8 specimens, AM P37218, $\mathrm{N}$ of Fly Point, Port Stephens, 32 $2^{\circ} 43^{\prime} \mathrm{S} 152^{\circ} 09^{\prime} \mathrm{E}, 20 \mathrm{~m}$, sponges, shell and sediment, R.T. Springthorpe \& D. Stracey, 8 November 1981, stn NSW-84. 3 specimens, AM P37219, Nelson Head, Port Stephens, 32 ${ }^{\circ} 43^{\prime} \mathrm{S}$ $152^{\circ} 09^{\prime} \mathrm{E}, 18 \mathrm{~m}$, bryozoans and hydroids, J. Hall, 27 October 1980, stn NSW-192. 7 specimens, AM P37220, same locality, 15-18 m, sand and shell grit, J. Hall \& I. Loch, 27 October 1980, stn NSW-193. 6 specimens, AM P37221, same locality, 20-24 m, rubble on stones, J. Hall, 27 October 1980, stn NSW-197. 1 specimen, AM P5719, off Red Rocks, Port Stephens, [approx. $32^{\circ} 42^{\prime} \mathrm{S} 152^{\circ} 06^{\prime} \mathrm{E}$ ], dredge, A. Musgrave, 30 August 1920. 4 specimens, AM P3441, Port Stephens, [approx. $32^{\circ} 42^{\prime} \mathrm{S}$ $152^{\circ} 06^{\prime}$ E]. 4 specimens, NMV J3331, Port Stephens. 1 specimen, AM P22054, E of North Head, Port Jackson, 33 $49^{\prime}$ S $151^{\circ} 18^{\prime} \mathrm{E}, 21 \mathrm{~m}$, with sponge Teichonella labyrinthica, 27 February 1973, AMSBS, stn 07. 4

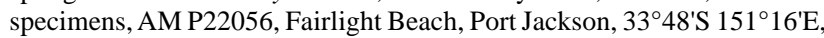
6-9 m, shell rubble, 26 May 1972, AMSBS. 4 specimens, AM P37222,

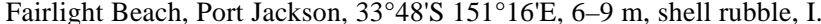
Loch, 28 February 1981, stn NSW-180. 8 specimens, AM P37223, SE of Chinamans Beach, Port Jackson, 33 $3^{\circ} 9^{\prime} \mathrm{S} 151^{\circ} 15^{\prime} \mathrm{E}, 9 \mathrm{~m}$, dead shells and sand, Malacological Society, 8 May 1971, stn NSW-182. 4 specimens, AM P37224, off Sow and Pigs, Port Jackson, 33 ${ }^{\circ} 50^{\prime} \mathrm{S} 151^{\circ} 15^{\prime} \mathrm{E}, 5 \mathrm{~m}$, shelly sand, J.K. Lowry \& A.R. Jones, on FV Port Jackson, 30 September 1976, stn NSW-184. 2 specimens, AM P37225, W of Spit Bridge, Port Jackson, $33^{\circ} 48^{\prime} \mathrm{S} 151^{\circ} 15^{\prime} \mathrm{E}, 8 \mathrm{~m}$, Tethya sponge and dead mussel shells, J.K. Lowry, 9 June 1981, stn NSW-34. 30 specimens, AM P3440, Port Jackson. 1 specimen, AM P5720, Port Jackson. 2 specimens, AM P5855, Port Jackson. 2 specimens, AM P37116, Clovelly Pool, 335ㄷ' $151^{\circ} 16^{\prime} \mathrm{E}$, 3-6 m, under stones, P.C. Terrill, 12 June 1979, stn NSW-210. 1 specimen, AM P37226, Botany Bay, [approx. $34^{\circ} 00^{\prime} \mathrm{S} 151^{\circ} 13^{\prime} \mathrm{E}$ ], NSW State Fisheries, 1975, stn NSW SF 899. 7 specimens, AM P37227, Botany Bay, [approx. $34^{\circ} 00^{\prime} S 151^{\circ} 13^{\prime} E$ ], NSW State Fisheries, 1975, stn NSW SF 901. 2 specimens, AM P37228, off Moona Moona Creek, Jervis Bay, $35^{\circ} 03.5^{\prime} \mathrm{S} 150^{\circ} 41^{\prime} \mathrm{E}, 3 \mathrm{~m}$, encrusting sponge, J.K. Lowry, 19 June 1982 , stn NSW-112. 1 specimen, AM P37229, same locality, 8 m, algae and sponge on sandy rocks and mussels, J.K. Lowry, 19 June 1982, stn NSW113. 8 specimens, AM P37230, same locality, $3 \mathrm{~m}$, Zonaria and encrusting ascidian in Ecklonia bed, J.K. Lowry, 19 June 1982, stn NSW-114. 2 specimens, AM P37231, same locality, 4 m, Ecklonia holdfast, P.B. Berents, 15 October 1981, stn NSW-265. 1 specimen, P37232, same locality, $4 \mathrm{~m}$, Ecklonia holdfast, P.B. Berents, September 1981, stn NSW266. 117 specimens, AM P37233 to P37241, same locality, 4.5 m, epifauna on test of solitary ascidian, Herdmania momus, in Ecklonia bed, P.B. Berents, October 1981-July 1982. 1 specimen, AM P37242, off Plantation Point, Jervis Bay, $35^{\circ} 04^{\prime} \mathrm{S} 150^{\circ} 42^{\prime} \mathrm{E}, 3 \mathrm{~m}$, red alga, Liagora, P.B. Berents, 21 February 1982, stn NSW-246. 169 specimens, AM P35982, P36702 and P37244 to P37248, Munganno Point, Twofold Bay, $37^{\circ} 06.2^{\prime} \mathrm{S}$ $149^{\circ} 55.7^{\prime} \mathrm{E}$, to $7 \mathrm{~m}$, sublittoral rock platform and wharf piles, S. Keable, October 1984-December 1985, stns FIRTA M3-M11. 35 specimens, AM P37249 to P37253, Murrumbulga Point, Twofold Bay, $37^{\circ} 04.7^{\prime} \mathrm{S} 149^{\circ} 53.1^{\prime} \mathrm{E}$, to $3 \mathrm{~m}$, sublittoral rock platform and breakwater wall, J. van der Velde \& S. Keable, October 1984-September 1985, stns FIRTA Q1-Q13.

Type locality. Port Jackson, New South Wales, Australia, Tasman Sea, [approx. $33^{\circ} 51^{\prime} \mathrm{S} 151^{\circ} 16^{\prime} \mathrm{E}$ ].

Description. Based on female, $11.0 \mathrm{~mm}$, AM P37116. Head much deeper than long, anterior margin with notch extended into a slit; rostrum absent; eye present, elongate, reniform. Antenna 1 peduncular article 1 not ball-shaped proximally, distal margin with small medial spine; peduncular article 2 medium length; flagellum with or without callynophore, calceoli absent. Antenna 2 flagellum about as long as that of antenna 1, without calceoli. Mouthpart bundle subconical. Epistome/upper lip almost straight (lateral view). Mandible lacinia mobilis a stemmed, distally-cusped blade; accessory setal row with intermediate setae; palp article 2 with 2 posterodistal setae, article 3 without A3-seta. Maxilliped outer plate with distal margin smooth, medial margin without notch.

Gnathopod 1 carpus shorter than propodus $(0.8 \times)$; propodus, posterior margin without robust setae. Gnathopod 2 palm slightly acute, with 1-3 lateral robust setae, 1 medial robust seta. Pereopods 3 and 4 merus and carpus without setal fringe. Pereopod 4 coxa with anterior and posterior margins subparallel, anteroventral corner rounded. Pereopods 5-7 with distal articles elongate, dactyls short and stocky. Pereopod 5 basis expanded posteriorly, rounded. Pereopod 7 basis rounded posteriorly, posteroventral corner rounded, posteroventral margin straight.

Epimeron 3 posterior margin smooth, with notch well above rounded posteroventral corner. Uropod 1 peduncle dorsolateral margin with 8 robust setae; outer ramus without large spines between robust setae. Uropod 2 inner ramus slightly constricted. Uropod 3 rami lanceolate; without plumose setae; outer ramus 1-articulate. Telson moderately cleft (about 40\%). 


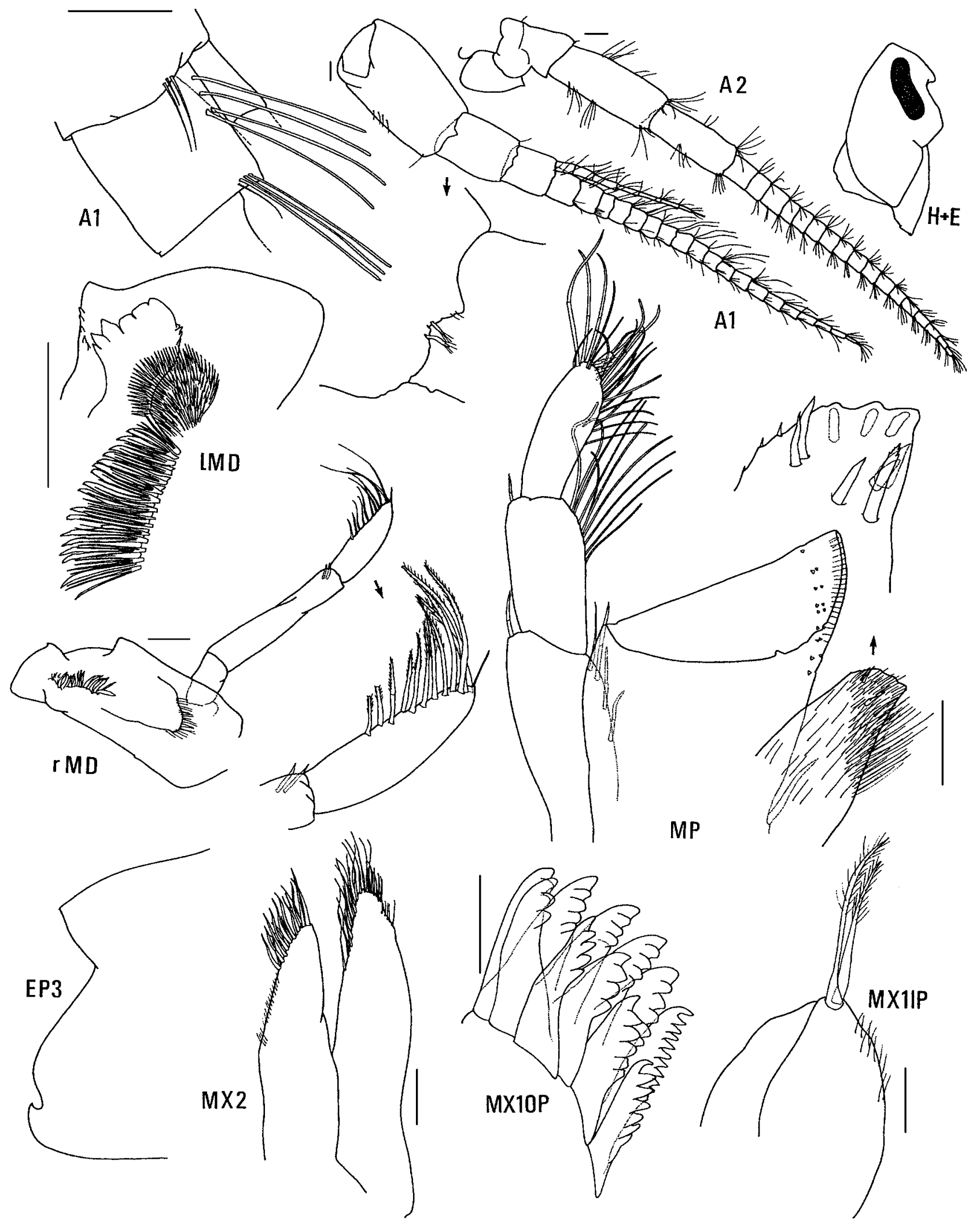

Figure 3. Amaryllis brevicornis Haswell, female, $11.0 \mathrm{~mm}$, AM P37116, Clovelly Pool, NSW. Scales for MX1 represent $0.05 \mathrm{~mm}$; remainder represent $0.1 \mathrm{~mm}$.

Variation. Amaryllis brevicornis, as we regard it here, shows more variation in the robust setae of gnathopod 2 palm than does any other species of Amaryllis. Adult females can be found with from 1 to 4 lateral (but always 1 medial) robust setae on the palm. There is also some variation in the arrangement of robust setae on the outer ramus of uropod 1. In some specimens, instead of regular spacing of the setae there is a distinct gap between the 1 or 2 proximal robust setae and the 1 or 2 distal robust setae. The variation in these characters does not occur consistently with either size 

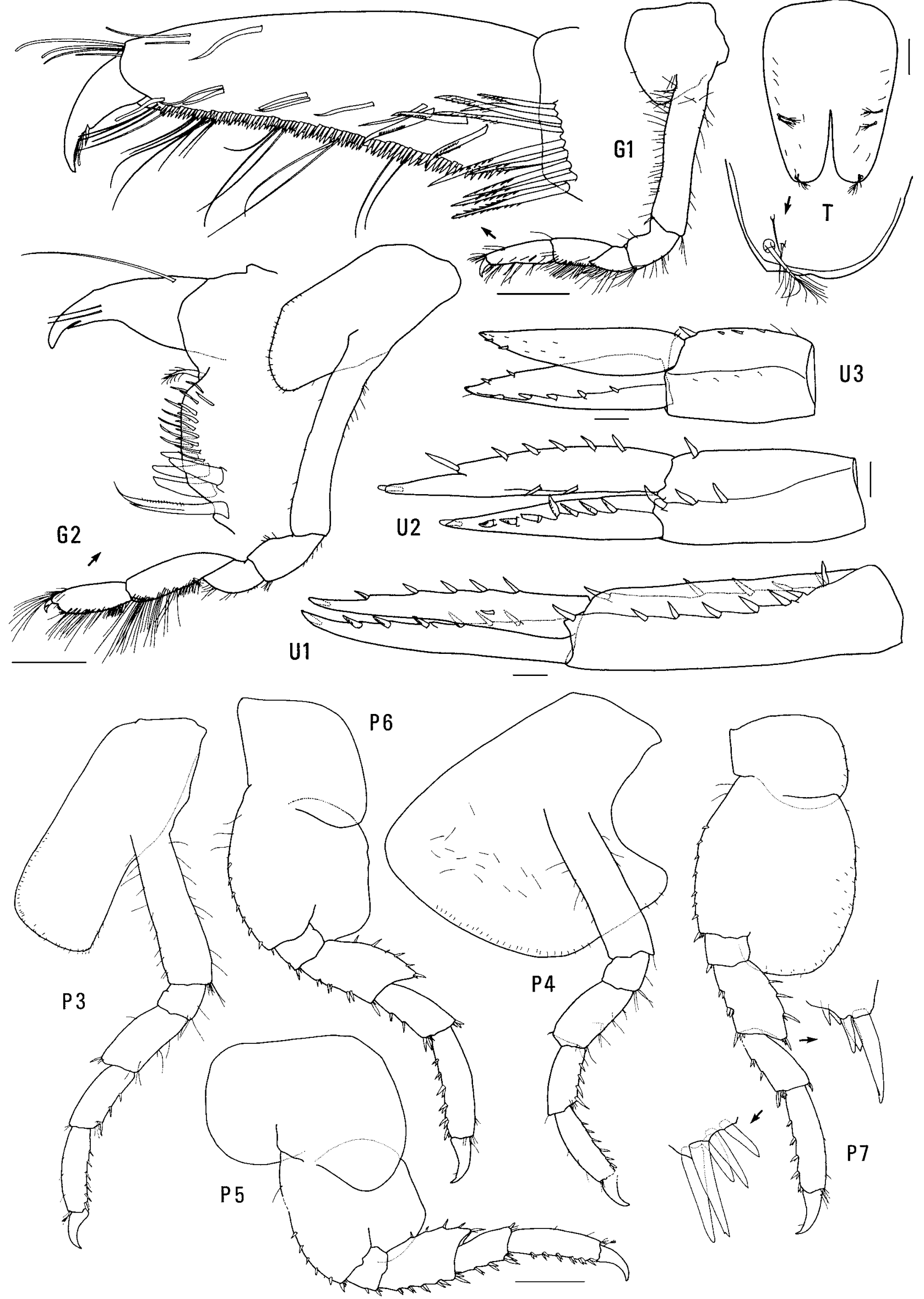

Figure 4. Amaryllis brevicornis Haswell, female, 11.0 mm, AM P37116, Clovelly, NSW. Scales for U1-3 and T represent $0.1 \mathrm{~mm}$, remainder represent $0.5 \mathrm{~mm}$. 
or geographical distribution, with any other character, or with each other. (A similar gap in the uropod 1 robust setae also occurs in A. migo, but in this case it consistently appears with other species characters.)

Remarks. Amaryllis brevicornis belongs to the group of species with a smooth posterior margin on epimeron 3 and a rounded posteroventral corner on the basis of pereopod 7. Within this group it is most similar to A. kamata, from which it differs in the shape of the epistome and the length of the gnathopod 1 carpus.

Habitat. Amaryllis brevicornis has been often collected with sponges; less often with other sessile invertebrates such as bryozoans, hydroids and ascidians, or in algal holdfasts, coralline algae and coarse rubble.

Distribution. Coastal New South Wales, southeastern Australia; to $24 \mathrm{~m}$ depth.

\section{Amaryllis carrascoi n.sp.}

Figs. 5-8, Plate 1a.

Amaryllis macrophthalma.-Chilton, 1921: 55 (in part).-J.L. Barnard, 1972: 262 (in part, figure of $8.5 \mathrm{~mm}$ female). Amaryllis sp. 2.-Hutchings et al., 1989: 362.

Type material. HoLOTYPE, female, $11.7 \mathrm{~mm}$, ovigerous (9 eggs), NMV J13954; 1 PARATYPE, male, 8.4 mm, NMV J13955; 30 PARATYPES, NMV J13956; 30 PARATYPES, AM P37117; 10 PARATYPES, SAMA C5971; $1 \mathrm{~km}$ off bay on north shore of Flinders Island, Investigator Group, South

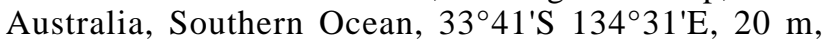
seagrass Posidonia, drift on sand, G.C.B. Poore, 19 April 1985, FV Lemnos, NMV stn SA-67.

Additional material. NeW South WALES: 1 specimen, AM P10858, small bay near Kurnell, Botany Bay, $34^{\circ} 00^{\prime} \mathrm{S} 151^{\circ} 12 \mathrm{E}$, sand and weed, F.A. McNeill, 27 October 1927. 4 specimens, AM P10849, Kurnell, Botany Bay, $34^{\circ} 00^{\prime} S 151^{\circ} 13^{\prime}$ E, F.A. McNeill, 6 October 1927.1 specimen, AM P37118, Moe's Rock, S of Jervis Bay, $35^{\circ} 09^{\prime} \mathrm{S} 150^{\circ} 45^{\prime} \mathrm{E}$, $6 \mathrm{~m}$, coralline algae Amphiroa anceps, P.B. Berents, 29 June 1981, stn NSW-58. 35 specimens, AM P37119, N side of Bawley Point, $35^{\circ} 31^{\prime} \mathrm{S}$ $150^{\circ} 24^{\prime} \mathrm{E}, 11 \mathrm{~m}$, coralline algae, P.B. Berents, 27 April 1981, stn NSW27. 1 specimen, AM P37120, same locality, $3 \mathrm{~m}$, coralline algae, P.D. Filmer-Sankey, 27 April 1981, stn NSW-26. 21 specimens, AM P36701, Murrumbulga Point, Twofold Bay, $37^{\circ} 04.7^{\prime} \mathrm{S} 149^{\circ} 53.1^{\prime} \mathrm{E}$, breakwater wall, 3 m, S. Keable \& P. Hutchings, 17 September 1985, stn FIRTA Q8. 1 specimen, AM P37121, Aslings Beach, Calle Calle Bay, Twofold Bay, $37^{\circ} 05^{\prime} \mathrm{S} 149^{\circ} 56^{\prime} \mathrm{E}, 8.5 \mathrm{~m}$, sediment, S. Keable \& P. Albertson, 22 February 1985, stn FIRTA EB6. 7 specimens, AM P37122, Quarantine Bay, Twofold Bay, $37^{\circ} 05^{\prime}$ S $149^{\circ} 56^{\prime} \mathrm{E}, 2 \mathrm{~m}$, seagrass Posidonia, S. Keable, 25 June 1985 , stn FIRTA Q8-10. VICTORIA: 36 specimens, NMV J11536, Duck Point, Corner Inlet, $38^{\circ} 49^{\prime} \mathrm{S} 146^{\circ} 16^{\prime} \mathrm{E}, 2 \mathrm{~m}$, seagrass Zostera on sand, corer, G.J. Morgan, 20 November 1983, stn CIN 24. 1 specimen, NMV J13957, Bennison Channel, Corner Inlet, $38^{\circ} 49^{\prime} \mathrm{S} 146^{\circ} 23^{\prime} \mathrm{E}, 6 \mathrm{~m}$, sand and shell grit, airlift, G.J. Morgan, 23 November 1983, stn CIN 28E. 13 specimens, NMV J3336, Shoreham, Western Port, [approx. $38^{\circ} 26^{\prime} \mathrm{S} 145^{\circ} 03^{\prime} \mathrm{E}$ ], O.A. Sayce Collection. 1 specimen, NMV J13958, Western Port, O.A. Sayce Collection. 1 specimen, NMV J13959, West Channel, O.A. Sayce Collection. 1 specimen, NMV J3235, Hobsons Bay, Port Phillip Bay, 3 $\mathrm{m}$, sandy shell, grab, stn PPBES 189. 1 specimen, NMV J13960, Port Phillip Bay. 8 specimens, NMV J13961, Port Phillip Bay, W. Williams, stn 25. 1 specimen, NMV J3247, Port Phillip Bay, 9-12 m, W. Williams, 2 April 1959. 1 specimen, NMV J3261, Port Phillip Bay, W. Williams, stn 64. 17 specimens, NMV J3269, Port Phillip Bay, 38 ${ }^{\circ} 14.0^{\prime} \mathrm{S} 144^{\circ} 39.5^{\prime} \mathrm{E}$, $1 \mathrm{~m}$, Venturi sampler, 23 January 1973, stn PPBES 966. 3 specimens, AM P58121, Lawrence Rocks, Portland, Victoria, $38^{\circ} 24^{\prime} \mathrm{S} 141^{\circ} 40.1^{\prime} \mathrm{E}$, $23 \mathrm{~m}$, bryozoan, possibly Orthoscuticella sp., R.T. Springthorpe \& P.B. Berents, 30 April 1988, stn VIC-49. BASS STRAIT: 10 specimens, AM
P58115, eastern Bass Strait, $38^{\circ} 56.5^{\prime} \mathrm{S} 148^{\circ} 19.3^{\prime} \mathrm{E}$, 80-85 m, epibenthic sled, P.B. Berents, 27 August 1994, FRV Southern Surveyor, stn SS 05/ 95/59. 3 specimens, AM P58118, eastern Bass Strait, 38 59.1'S $148^{\circ} 31.6^{\prime} \mathrm{E}, 125 \mathrm{~m}$, epibenthic sled, P.B. Berents, 27 August 1994, FRV Southern Surveyor, stn SS 05/95/60. 6 specimens, NMV J13963, 9 km SSW of Cape Adansan, Three Hummock Island, central Bass Strait, $40^{\circ} 30.9^{\prime} \mathrm{S} 144^{\circ} 56^{\prime} \mathrm{E}, 27 \mathrm{~m}$, very coarse sand, epibenthic sled, M. Gomon \& G.C.B. Poore, 3 November 1980, FRV Sarda, stn BSS-109. 6 specimens, NMV J13987, $5 \mathrm{~km}$ E of Cape Edie, Robbins Island, Central Bass Strait, $40^{\circ} 41.8^{\prime} \mathrm{S} 145^{\circ} 07^{\prime} \mathrm{E}, 16 \mathrm{~m}$, fine shelly sand, epibenthic sled, M.Gomon \& G.C.B. Poore, 3 November 1980, FRV Sarda, stn BSS-110. TASMANIA: 1 specimen, AM P37123, off St Helens Point, $41^{\circ} 20.6^{\prime} \mathrm{S}$ $148^{\circ} 30^{\prime} \mathrm{E}, 110 \mathrm{~m}$, fine clayey sand, P.H. Colman, 25 March 1973, MV Sprightly, stn BMR S73-2038. 1 specimen, AM P37124, Bicheno, 41 ${ }^{\circ} 53$ 'S $148^{\circ} 18^{\prime}$ E, 20-30 m, bryozoans and Macrocystis holdfast, R.H. Kuiter, 8 May 1986, AM stn TAS-26. 4 specimens, NMV J13962, Coles Bay, $42^{\circ} 07^{\prime} \mathrm{S} 148^{\circ} 17^{\prime} \mathrm{E}, 1 \mathrm{~m}$, red algae and invertebrates on rock wall, airlift, R.S. Wilson, 21 April 1985, NMV stn TAS-17. 110 specimens, AM P58120, off rocky point near eastern end of Bryans Beach, Freycinet Peninsula, Tasmania, $42^{\circ} 16^{\prime} \mathrm{S} 148^{\circ} 17.4^{\prime} \mathrm{E}, 12 \mathrm{~m}$, baited trap, probably sandy bottom, J.K. Lowry \& S.J. Keable, 30 April-1 May 1991, AM stn TAS-338. 7 specimens, AM P27052, Shouten Passage, $42^{\circ} 17^{\prime} \mathrm{S} 148^{\circ} 18^{\prime} \mathrm{E}$, $12 \mathrm{~m}$, coarse sand and coralline algae, van Veen grab, A.J. Dartnall, 8 June 1977, FRV Penghana, stn 46. 1 specimen, NMV J13984, $5 \mathrm{~km}$ NE of Mistaken Cape, Maria Island, $42^{\circ} 37^{\prime} \mathrm{S} 148^{\circ} 12.5^{\prime} \mathrm{E}, 100 \mathrm{~m}$, "fine muddy bryozoans", epibenthic sled, R.S. Wilson, 23 April 1985, RV Challenger, NMV stn TAS-31. 1 specimen, AM P37125, Tasmanian coast, FIS Endeavour Collection. SOUTH AUSTRALIA: 2 specimens, NMV J13964, $\mathrm{NE}$ end of West Island, Encounter Bay, 35 37'S 138 $36^{\prime} \mathrm{E}, 12 \mathrm{~m}$, red algae, G.C.B. Poore \& H.M. Lew Ton, 20 March 1985, NMV stn SA-39. 1 specimen, NMV J13965, N side of West Island, Encounter Bay, $35^{\circ} 37^{\prime} \mathrm{S}$ $138^{\circ} 36^{\prime} \mathrm{E}, 5 \mathrm{~m}$, coralline algae, G.C.B. Poore \& H.M. Lew Ton, 21 March 1985, NMV stn SA-43. 14 specimens, NMV J13966, same locality, 5 m, sandy sediment, G.C.B. Poore \& H.M. Lew Ton, 21 March 1985, NMV stn SA-46. 1 specimen, AM P37126, Marino, Gulf St Vincent, [approx. $\left.35^{\circ} 03^{\prime} \mathrm{S} 138^{\circ} 31^{\prime} \mathrm{E}\right]$, W.H. Baker, 1910. 2 specimens, AM P37127, Sellicks Beach, Gulf St Vincent, [approx. $35^{\circ} 20^{\prime} \mathrm{S} 138^{\circ} 27^{\prime}$ E] , H.M. Hale, 1936. 1 specimen, SAMA C5972, Dangerous Reef, Spencer Gulf, [approx. 34²9'S $\left.136^{\circ} 12^{\prime} \mathrm{E}\right]$, dredged, K. Sheard. 2 specimens, SAMA C5973, Marion Bay, Yorke Peninsula, [approx. $35^{\circ} 14^{\prime} \mathrm{S} 137^{\circ} 00^{\prime} \mathrm{E}$ ], $15 \mathrm{~m}$, on bait in craypots, W. Zeidler, 28 January 1980. 2 specimens, NMV J13967, The Hotspot reef, 5 nautical miles $\mathrm{W}$ of $\mathrm{N}$ end of Flinders Island, $33^{\circ} 40.5^{\prime} \mathrm{S}$ $134^{\circ} 22.0^{\prime} \mathrm{E}, 17 \mathrm{~m}$, tufted bryozoan on exposed rock face, G.C.B. Poore, 19 April 1985, FV Lemnos, NMV stn SA-62. 2 specimens, NMV J13983, same locality, $17 \mathrm{~m}$, tufted red alga on flat exposed rock face, G.C.B. Poore, 19 April 1985, FV Lemnos, NMV stn SA-63. 26 specimens, NMV $\mathrm{J} 13968$, same locality, $12 \mathrm{~m}$, mixed large forms of brown, red and green algae, S. Shepherd, 19 April 1985, FV Lemnos, NMV stn SA-64. 9 specimens, NMV J13969, same locality, $17 \mathrm{~m}$, mixed large forms of brown, red and green algae, S. Shepherd, 19 April 1985, FV Lemnos, NMV stn SA-65. 5 specimens, NMV J13970, The Hotspot reef, 5 nautical miles $\mathrm{W}$ of $\mathrm{N}$ end of Flinders Island, $33^{\circ} 40.8^{\prime} \mathrm{S} 134^{\circ} 22.5^{\prime} \mathrm{E}, 21 \mathrm{~m}$, tufted red algae and soft erect bryozoan, G.C.B. Poore, 29 April 1985, FV Lemnos, NMV stn SA-72. 4 specimens, NMV J13971, $1 \mathrm{~km}$ off bay on $\mathrm{N}$ shore of Flinders Island, $33^{\circ} 41^{\prime} \mathrm{S} 134^{\circ} 31^{\prime} \mathrm{E}$, drift algae on sand, hand dredge, G.C.B. Poore, 19 April 1985, NMV stn SA-68. 1 specimen, NMV $\mathrm{J} 13972$, NE coast of Topgallant Island, Investigator Group, 33 $43.0^{\prime} \mathrm{S}$ $134^{\circ} 36.6^{\prime} \mathrm{E}, 16 \mathrm{~m}$, brown alga Cystophora sp., S. Shepherd \& G.C.B. Poore, 22 April 1985, NMV stn SA-84. 7 specimens, NMV J13973, Venus Bay, S side of Germein Island, $33^{\circ} 13.2^{\prime} \mathrm{S} 134^{\circ} 40.1^{\prime} \mathrm{E}, 2 \mathrm{~m}$, shelly sand flat, hand dredge, G.C.B. Poore, 23 April 1985, NMV stn SA-87. 1 specimen, NMV J13974, same data, NMV stn SA-88. 1 specimen, AM P37396, off St Francis Island, [approx. 32 ${ }^{\circ} 31^{\prime} \mathrm{S} 133^{\circ} 18^{\prime} \mathrm{E}$ ], 11-24 m, J.C. Verco. WeStern Australia: 1 specimen, AM P37128, SE of South Point, Two Peoples Bay, $34^{\circ} 58^{\prime} \mathrm{S} 118^{\circ} 12^{\prime} \mathrm{E}, 12 \mathrm{~m}$, bryozoan Orthoscuticella sp., R.T. Springthorpe, 16 December 1983, stn WA-182. 1 specimen, AM P37129, same locality, $12 \mathrm{~m}$, coralline and green algae, sea grasses, R.T. Springthorpe, 16 December 1983, stn WA-183. 1 specimen, AM P37130, Mistaken Island, Vancouver Peninsula, King George Sound, $35^{\circ} 04^{\prime} \mathrm{S}$ $117^{\circ} 56^{\prime} \mathrm{E}, 3 \mathrm{~m}$, stalked ascidian, J.K. Lowry, 13 December 1983, stn WA109. 1 specimen, AM P37131, off Possession Point, King George Sound, $35^{\circ} 02.5^{\prime} \mathrm{S} 117^{\circ} 55^{\prime} \mathrm{E}, 10 \mathrm{~m}$, sand and detritus from bases of sea grasses, R.T. Springthorpe \& J.K. Lowry, 14 December 1983, stn WA-131. 1 specimen, AM P37132, same locality, $7 \mathrm{~m}$, algae, bryozoan and sponge, R.T. Springthorpe \& J.K. Lowry, 14 December 1983, stn WA-133. 1 specimen, NMV J13975, N of False Island, King George Sound, 35 $00.7^{\prime} \mathrm{S}$ $118^{\circ} 10.1^{\prime} \mathrm{E}, 27 \mathrm{~m}$, red algae and bryozoan, G.C.B. Poore \& H.M. Lew Ton, 15 April 1985, NMV stn SWA-57. 
Type locality. One kilometre off bay on north shore of Flinders Island, Investigator Group, South Australia, $33^{\circ} 41^{\prime} \mathrm{S} 134^{\circ} 31^{\prime} \mathrm{E}, 20 \mathrm{~m}$ depth.

Description. Based on holotype female, $11.7 \mathrm{~mm}$, NMV J13954. Head much deeper than long, anterior margin with notch extended into a slit; rostrum absent; eye present, elongate, reniform. Antenna 1 peduncular article 1 not ballshaped proximally, distal margin with small medial spine; peduncular article 2 medium length; flagellum without callynophore, calceoli absent. Antenna 2 flagellum about as long as that of antenna 1, without calceoli. Mouthpart bundle subconical. Epistome/upper lip almost straight (lateral view). Mandible lacinia mobilis a stemmed, distallycusped blade; accessory setal row with intermediate setae; palp article 2 with 2 posterodistal setae, article 3 without A3-seta. Maxilliped outer plate with distal margin smooth, medial margin without notch.

Gnathopod 1 carpus subequal in length to propodus $(0.9 \times)$; propodus, posterior margin without robust setae. Gnathopod 2 palm slightly acute, with 3 lateral robust setae, 3 medial robust setae. Pereopods 3 and 4 merus and carpus without setal fringe. Pereopod 4 coxa with anterior and posterior margins subparallel, anteroventral corner rounded. Pereopods 5-7 with distal articles elongate, dactyls short and stocky. Pereopod 5 basis expanded posteriorly, rounded. Pereopod 7 basis subrectangular, posteroventral corner notched, posteroventral margin straight.

Epimeron 3 posterior margin serrate, with notch well above rounded posteroventral corner. Uropod 1 peduncle dorsolateral margin with 9 robust setae; outer ramus without large spines between robust setae. Uropod 2 inner ramus slightly constricted. Uropod 3 rami lanceolate; without plumose setae; outer ramus 1 -articulate. Telson moderately cleft (about 40\%).

Male (sexually dimorphic characters). Based on paratype male, 8.4 mm, NMV J13955. Antenna 1 flagellum with callynophore, with calceoli. Antenna 2 flagellum with calceoli. Mandible palp article 2 with 8 posterodistal setae. Gnathopod 2 palm with 1 lateral robust seta and 1 medial robust seta.

Variation. There is some minor variation in the appearance of epimeron 3 in A. carrascoi. In small specimens there may be as few as 2 small serrations above the notch on the posterior margin. Although always present, the notch itself is not well developed in some specimens, in which case the margin may appear to be only serrate. However, in Amaryllis species with a serrate posterior margin on epimeron 3 , the serrations begin at the ventral margin whereas in species with a notch and serrations, the notch is well above the rounded posteroventral corner and the additional small serrations occur above the notch.

Etymology. Named for Jacque Carrasco, a Colombian natural history artist who lived and died in Australia before many of his ideas, integrating art and conservation, were ever realised.

Remarks. Amaryllis carrascoi is similar to other Amaryllis species with a serrate posterior margin on epimeron 3 , such as A. croca, A. dianae, A. keablei and A. migo. It differs from all other species in this group in having a notched posteroventral corner on the basis of pereopod 7 .

Habitat. Amaryllis carrascoi has been most frequently collected in association with coralline algae, but also with other types of algae, sea grasses and bryozoans; or on fine to coarse sands. It has twice been taken in baited traps.

Distribution. Southern Australia; from Botany Bay on the southeastern coast, through Tasmanian, Victorian and South Australian coasts to King George Sound in southern Western Australia. Except for a few records from $80-125 \mathrm{~m}$ off eastern Bass Strait and the Tasmanian coast, it occurs in shallow water, 1-30 m depth.

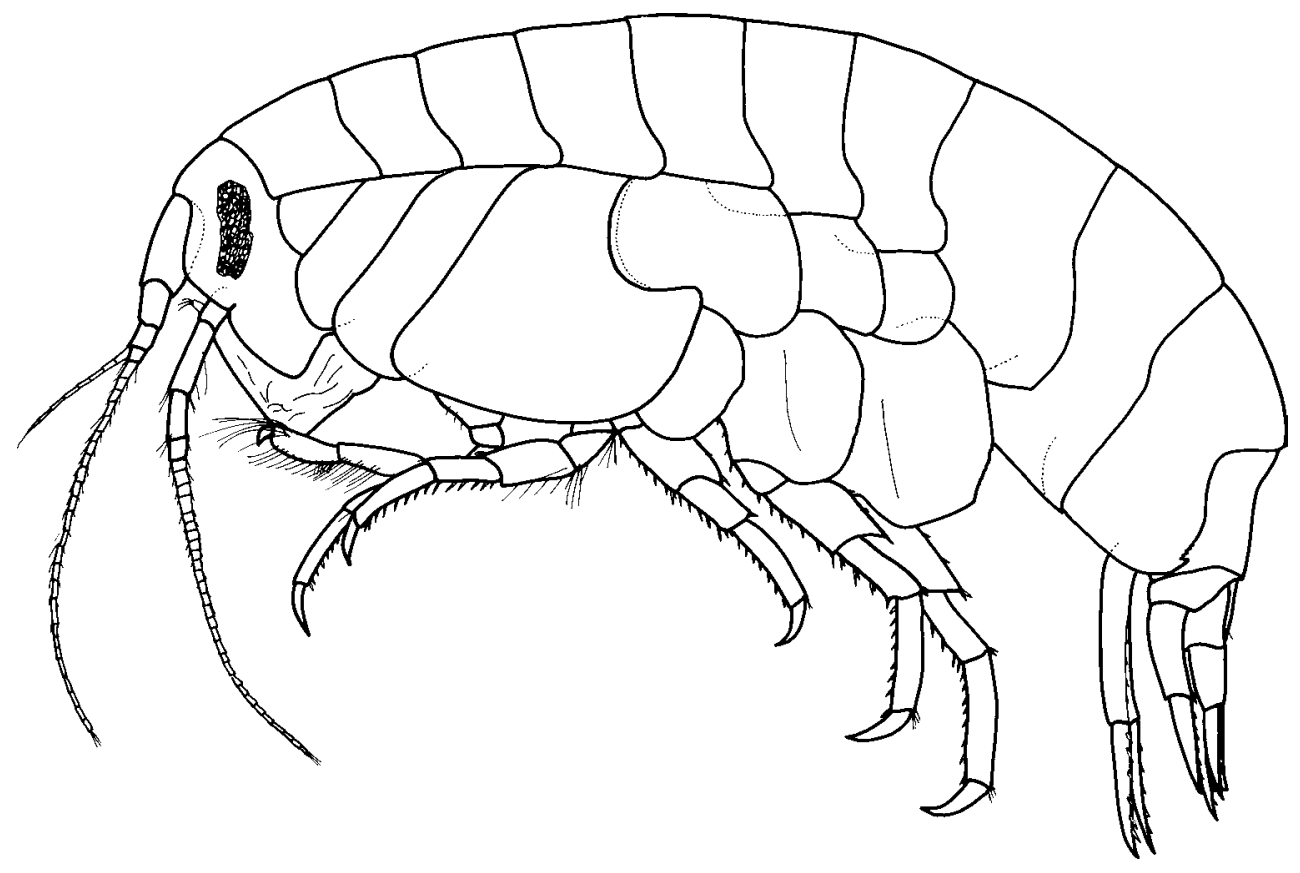

Figure 5. Amaryllis carrascoi n.sp., holotype female, 11.7 mm, NMV J13954, Flinders Island, South Australia. 


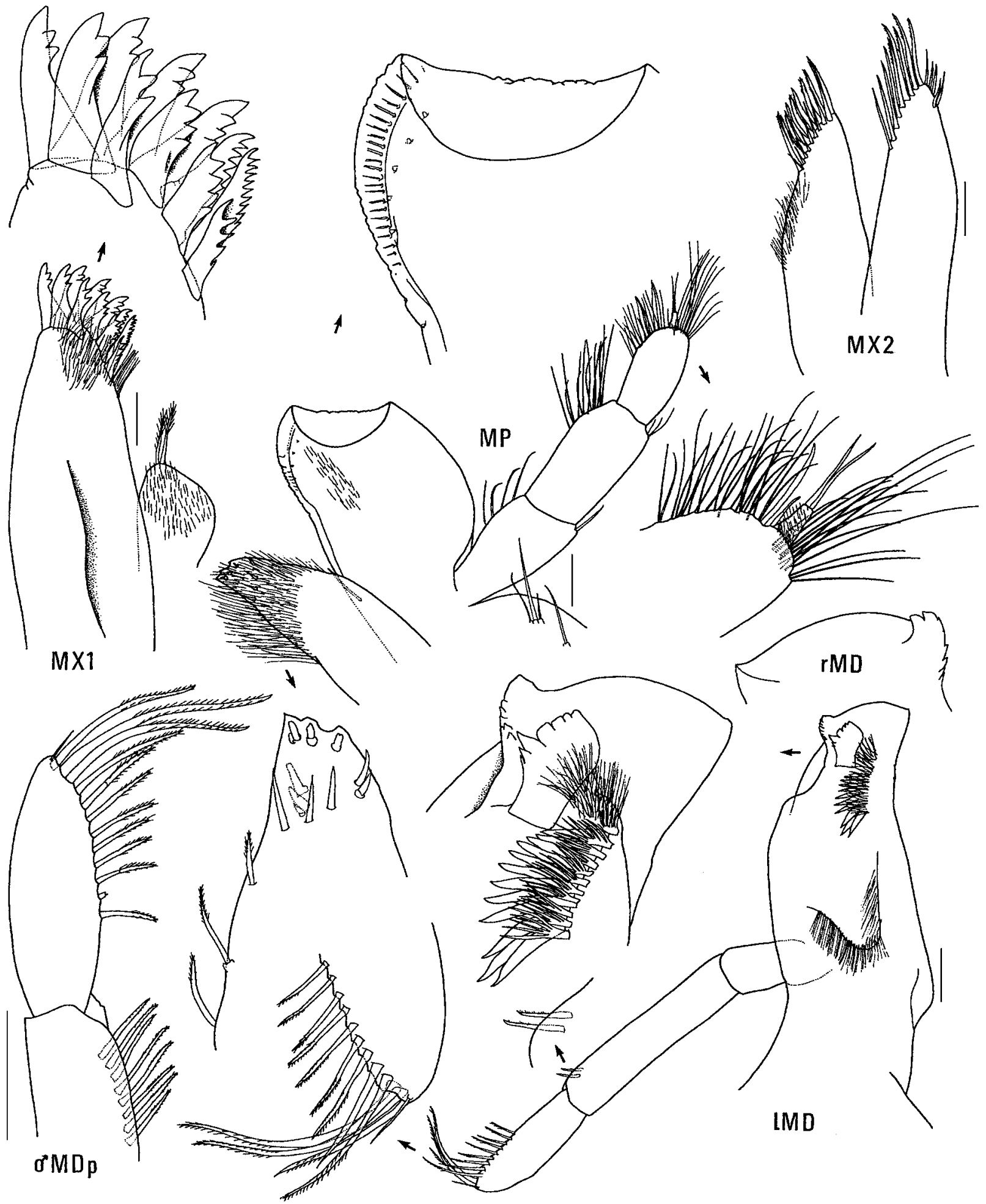

Figure 6. Amaryllis carrascoi n.sp., holotype female, 11.7 mm, NMV J13954; paratype male, 8.4 mm, NMV J13955; Flinders Island, South Australia. Scales represent $0.1 \mathrm{~mm}$. 


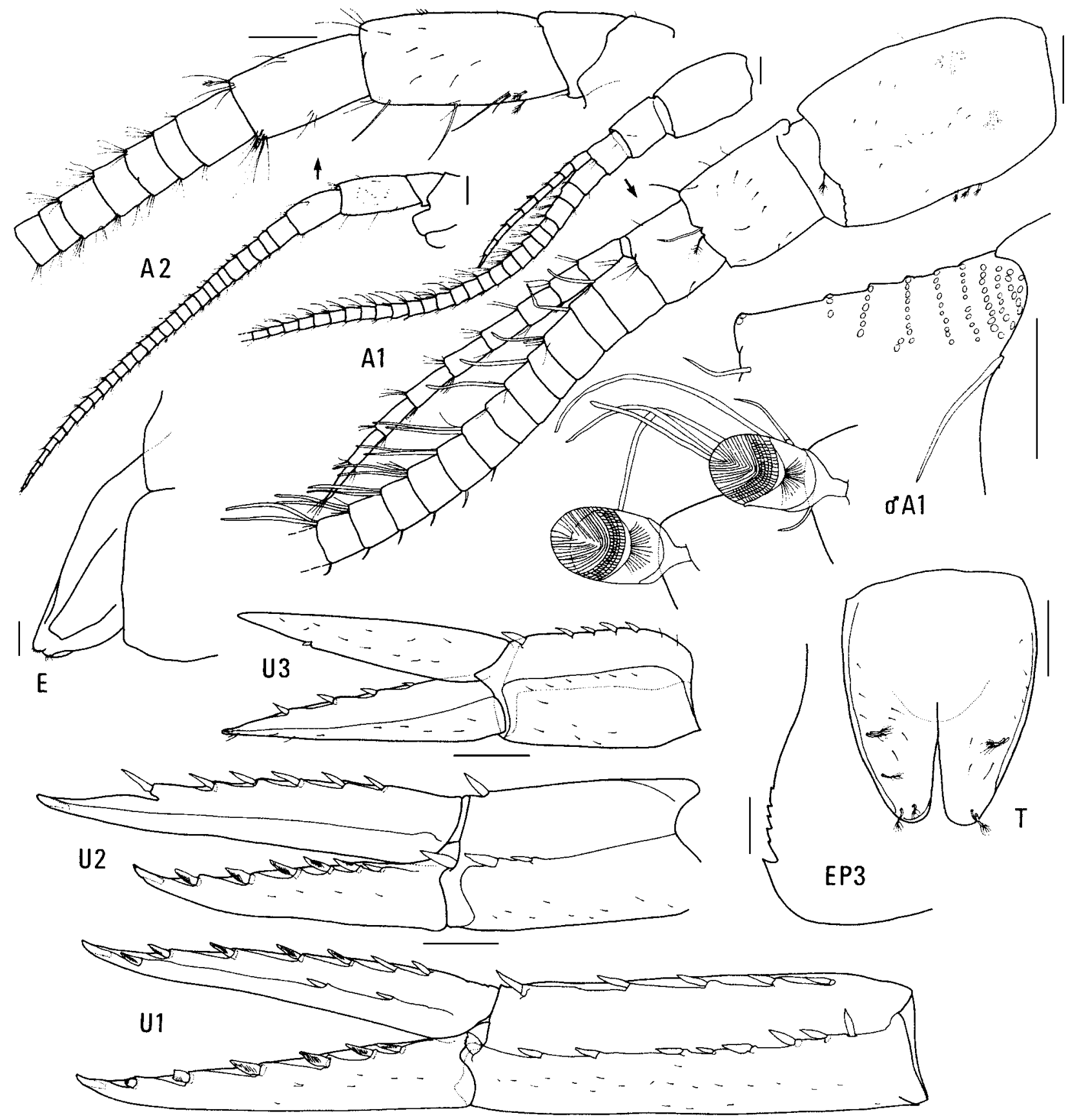

Figure 7. Amaryllis carrascoi n.sp., holotype female, $11.7 \mathrm{~mm}$, NMV J13954; paratype male, $8.4 \mathrm{~mm}$, NMV J13955; Flinders Island, South Australia. Scales represent $0.2 \mathrm{~mm}$. 


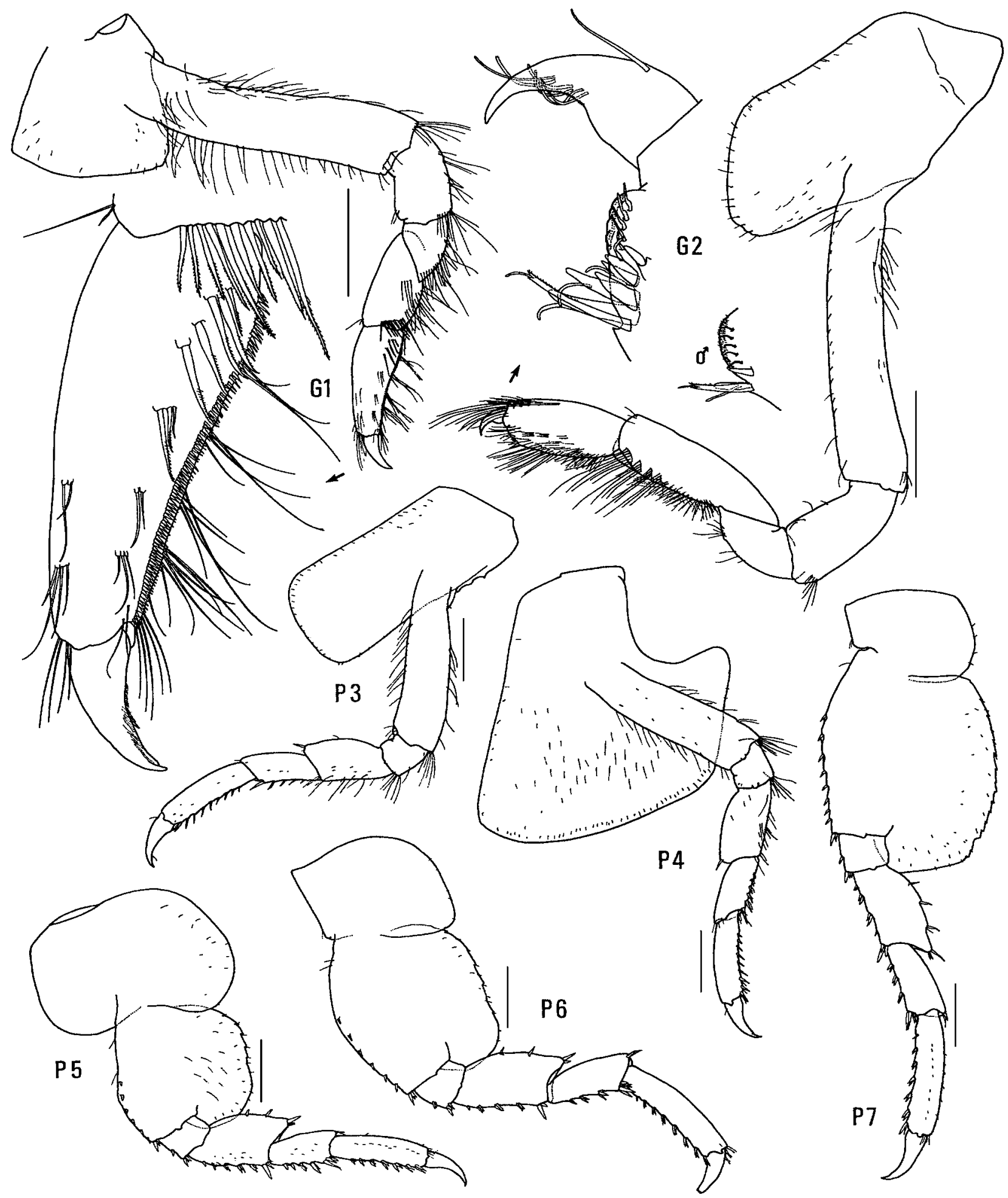

Figure 8. Amaryllis carrascoi n.sp., holotype female, 11.7 mm, NMV J13954; paratype male, 8.4 mm, NMV J13955; Flinders Island, South Australia. Scales represent $0.5 \mathrm{~mm}$. 

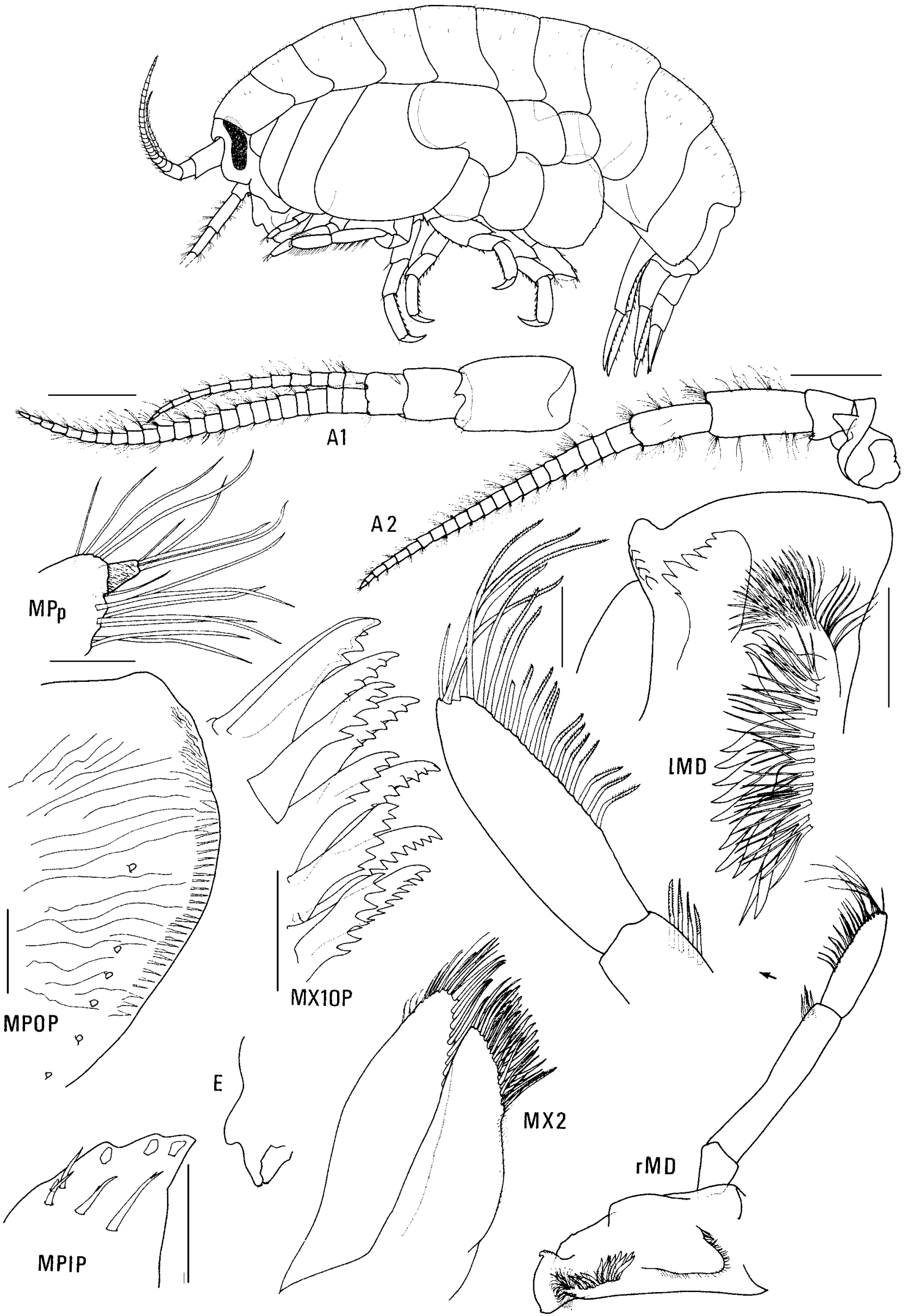


\section{Amaryllis croca n.sp.}

Figs. 9, 10

Amaryllis macrophthalma.-Chilton, 1921: 55 (in part).

Type material. HolOTYPe, female, $16.5 \mathrm{~mm}$, ovigerous (40 eggs), NMV J13976, The Hotspot reef, 5 nautical miles west of Flinders Island, South Australia, Southern Ocean, $33^{\circ} 40.8^{\prime} \mathrm{S} 134^{\circ} 22.5^{\prime} \mathrm{E}, 21 \mathrm{~m}$, erect hard bryozoan on vertical rock face, G.C.B. Poore \& H.M. Lew Ton, 20 April 1985, NMV stn SA-71.

Additional material. Victoria: 2 females, NMV J3791, Western Port, 11 May 1968, stn B/1/20. 1 female, NMV J13977, Western Port, 7 April 1968, stn B/1/9. South Australia: 1 female, AM E6525, $24 \mathrm{~km} \mathrm{NW}$ of Cape Jarvis, Gulf St Vincent, [approx. $35^{\circ} 25^{\prime} \mathrm{S} 137^{\circ} 55^{\prime} \mathrm{E}$ ], $31 \mathrm{~m}$, FIS Endeavour collection. 2 specimens, immature, AM P37179, Rapid Bay, Gulf St Vincent, $35^{\circ} 31^{\prime} S 138^{\circ} 11^{\prime} \mathrm{E}$, on jetty piles, P. Hutchings, 7 March 1979.

Type locality. The Hotspot reef, 5 nautical miles west of Flinders Island, South Australia, Southern Ocean, $33^{\circ} 40.8^{\prime} \mathrm{S}$ $134^{\circ} 22.5^{\prime} \mathrm{E}, 21 \mathrm{~m}$ depth.

Description. Based on holotype female, $16.5 \mathrm{~mm}$, NMV J13976. Head much deeper than long, anterior margin with notch extended into a slit; rostrum absent; eye present, elongate, reniform. Antenna 1 peduncular article 1 not ballshaped proximally, distal margin with well-developed medial spine; peduncular article 2 medium length; flagellum without callynophore, calceoli absent. Antenna 2 flagellum about as long as that of antenna 1, without calceoli. Mouthpart bundle subconical. Epistome/upper lip with a mid-anterior hook (lateral view). Mandible lacinia mobilis a stemmed, distally-cusped blade; accessory setal row with intermediate setae; palp article 2 with 4 posterodistal setae, article 3 without A3-seta. Maxilliped outer plate with distal margin smooth, medial margin without notch.

Gnathopod 1 carpus subequal in length to propodus; propodus, posterior margin without robust setae. Gnathopod 2 palm slightly acute, with 1 lateral robust seta, 3-4 medial robust setae. Pereopods 3 and 4 merus and carpus without setal fringe. Pereopod 4 coxa with anterior and posterior margins subparallel, anteroventral corner rounded. Pereopods 5-7 with distal articles elongate, dactyls short and stocky. Pereopod 5 basis expanded posteriorly, rounded. Pereopod 7 basis subrectangular, posteroventral corner subquadrate, posteroventral margin straight.

Epimeron 3 posterior margin serrate, without notch. Uropod 1 peduncle dorsolateral margin with 10 robust setae; outer ramus without large spines between robust setae. Uropod 2 inner ramus slightly constricted. Uropod 3 rami lanceolate; without plumose setae; outer ramus 1-articulate. Telson moderately cleft (about 45\%).

Etymology. The specific name croca is a Medieval Latin word for a shepherd's crook, an allusion to the hooked epistome of this species.

Remarks. Amaryllis croca is similar to other Amaryllis species with a serrate posterior margin on epimeron 3 , such as A. carrascoi, A. dianae, A. keablei and A. migo. It is most similar to A. keablei, in that the serrations extend to the posteroventral corner. Amaryllis croca differs from A. keablei in having a well-developed medial spine on peduncular article 1 of antenna 1 , posterodistal setae on article 2 of the female mandibular palp and one lateral robust seta on the palm of gnathopod 2. Amaryllis croca differs from all other Amaryllis species in having a midanterior hook on the epistome/upper lip.

Habitat. Amaryllis croca is known from bryozoans on a rock face and on wharf pilings.

Distribution. Southeastern coast of Australia; 21-31 m depth.

\section{Amaryllis dianae n.sp.}

$$
\text { Figs. 11-13 }
$$

Amaryllis macrophthalma.-Chilton, 1921: 55 (in part).

Type material. HоLOTYPE, female, 16.5 mm, AM P37378, off South Mole, Arthur Head, Fremantle, Western Australia, eastern Indian Ocean, $32^{\circ} 03^{\prime} \mathrm{S} 115^{\circ} 44^{\prime} \mathrm{E}, 6 \mathrm{~m}$, spirorbid worm tubes, J.K. Lowry, 25 December 1983, stn WA-283. 1 PARATYPE, female, AM P37379, type locality, $6 \mathrm{~m}$, orange gorgonian, R.T. Springthorpe, 25 December 1983, stn WA286. 2 PARATYPES, male, AM P37380, Cathedral Rock, Rottnest Island, Western Australia, $32^{\circ} 01.5^{\prime} \mathrm{S} 115^{\circ} 27^{\circ} \mathrm{E}, 3$ m, orange tunicates, J.K. Lowry, 21 December 1983, stn WA-247. 1 PARATYPE, AM P37381, same locality, $3 \mathrm{~m}$, coralline algae, some red algae and sponge, J.K. Lowry, 21 December 1983, stn WA-248. 1 PARATYPE, male, AM P37382 and 3 PARATYPES, female, WAM C28202, same locality, $6 \mathrm{~m}$, mixed algae, R.T. Springthorpe, 21 December 1983, stn WA-251.

Additional material. Victoria: 1 specimen, AM P58122, Lawrence Rocks, Portland, $38^{\circ} 24^{\prime} \mathrm{S} 141^{\circ} 40.1^{\prime} \mathrm{E}, 23 \mathrm{~m}$, bryozoan, possibly Orthoscuticella, R.T. Springthorpe \& P.B. Berents, 30 April 1988, stn VIC-49. South Australia: 1 specimen, NMV J48799, NE end of West Island, Encounter Bay, $35^{\circ} 37^{\prime} \mathrm{S} 138^{\circ} 36^{\prime} \mathrm{E}, 15 \mathrm{~m}$, red algae, sponges and bryozoans, G.C.B. Poore \& H.M. Lew Ton, 20 March 1985, NMV stn SA-42. 1 specimen, AM P37383, 8-10 km off Semaphore, Gulf St Vincent, [approx. $34^{\circ} 52^{\prime} \mathrm{S} 138^{\circ} 20^{\prime} \mathrm{E}$ ], 9 m, H.M. Hale, 1925. 2 specimens AM P37384, Marino, Gulf St Vincent, [approx. $35^{\circ} 03^{\prime} \mathrm{S} 138^{\circ} 31^{\prime} \mathrm{E}$ ], W.H. Baker, 1910. 2 specimens, SAMA C5974, $20 \mathrm{~km}$ due W of Outer Harbour, Adelaide, [approx. $34^{\circ} 45^{\prime} \mathrm{S} 138^{\circ} 15^{\prime} \mathrm{E}$ ], 23-25 m, quadrat G8, J. McPhalain, 17 April 1975. 2 specimens, SAMA C5975, same locality, quadrat H4, J. McPhalain, 22 April 1975. 1 specimen, SAMA C5976, same locality, quadrat G6, J. McPhalain, 17 April 1975. 1 specimen, SAMA C5977, same locality, quadrat H7, J. McPhalain, 22 April 1975. 1 specimen, SAMA C5978, Tiparra Reef, Spencer Gulf, S.A., [approx. $34^{\circ} 03^{\prime} \mathrm{S}$ $\left.137^{\circ} 24^{\prime} \mathrm{E}\right], 5 \mathrm{~m}$, in seagrass Posidonia, S.A. Shepherd. 2 specimens, SAMA C5979, The Pages, [approx. 35 $47^{\circ} \mathrm{S} 138^{\circ} 17^{\prime} \mathrm{E}$ ], $27 \mathrm{~m}$, dredged, K. Sheard, 12 April 1941. 1 specimen, AM P37385, Nepean Bay, Kangaroo Island, [approx. 3542'S 137³7'E], F. Moorhouse, May 1938. 1 specimen, AM P37386, unknown locality, Gulf St Vincent. 2 specimens, AM P37387, behind Lowly Point, Spencer Gulf, [approx. 3300'S $\left.137^{\circ} 46^{\prime} \mathrm{E}\right], 18 \mathrm{~m}$, K. Sheard on FL Whyalla, 7 March 1938. 1 specimen, AM P37388, Western Shoal, Spencer Gulf, [approx. $33^{\circ} 09^{\prime} \mathrm{S} 137^{\circ} 31^{\prime} \mathrm{E}$ ], $9 \mathrm{~m}, \mathrm{~K}$. Sheard on FL Whyalla, 10 March 1938. 1 specimen, NMV J13982, The Hotspot Reef, W of Flinders Island, $33^{\circ} 40.8^{\prime} \mathrm{S} 134^{\circ} 22.5^{\prime} \mathrm{E}, 21 \mathrm{~m}$, erect hard bryozoan on vertical rock face, G.C.B. Poore, 20 April 1985,

Figure 9. Amaryllis croca n.sp., holotype female, 16.5 mm, NMV J13976, The Hotspot Reef, South Australia. Scales for A1, 2 represent $0.5 \mathrm{~mm}$; remainder represent $0.1 \mathrm{~mm}$. 


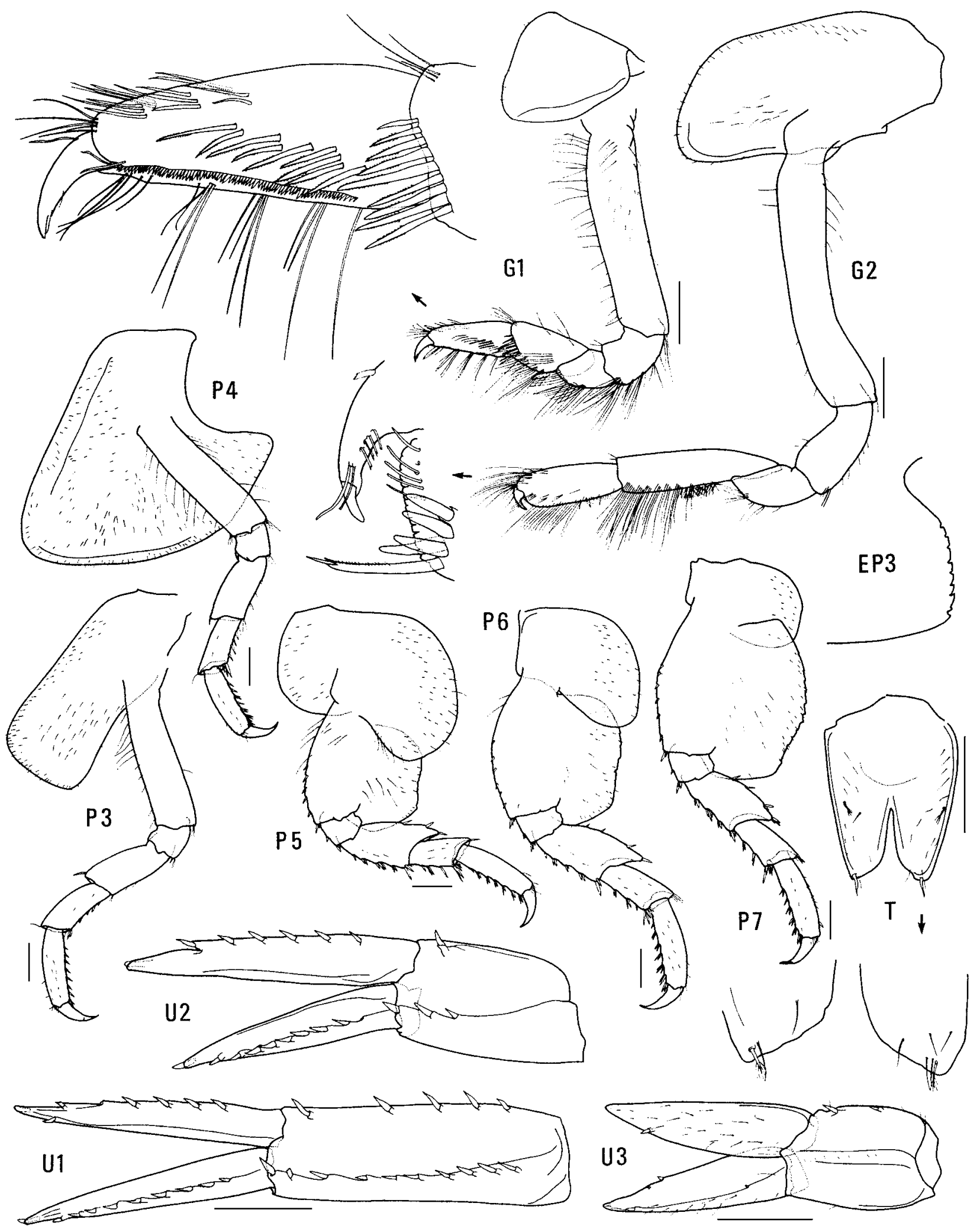

Figure 10. Amaryllis croca n.sp., holotype female, 16.5 mm, NMV J13976, The Hotspot Reef, South Australia. Scales represent $0.5 \mathrm{~mm}$.

NMV stn SA-71. WeStern Australia: 1 specimen, AM P18313, SW of Eucla, Great Australian Bight, 146-219 m, FIS Endeavour. 3 specimens, AM P37389, 2 km SE of South Point, Two Peoples Bay, $34^{\circ} 58^{\prime} \mathrm{S} 118^{\circ} 12^{\prime} \mathrm{E}, 14 \mathrm{~m}$, among green alga Caulerpa bases and fine sand, J.K. Lowry, 16 December 1983, stn WA-178. 5 specimens, AM P37390, rocks near Migo Island, Port Harding, Torbay Bay, $35^{\circ} 04^{\prime} \mathrm{S} 117^{\circ} 39^{\prime} \mathrm{E}, 6$ $7 \mathrm{~m}$, sponge and algae, R.T. Springthorpe, 15 December 1983, stn WA-
145. 1 specimen, AM P37391, Geographe Bay, [approx. 33³7'S $\left.115^{\circ} 18^{\prime} \mathrm{E}\right], 27-29 \mathrm{~m}, \mathrm{~J} . \mathrm{C}$. Verco.

Type locality. Off South Mole, Arthur Head, Fremantle, Western Australia, eastern Indian Ocean, $32^{\circ} 03^{\prime} \mathrm{S}$ $115^{\circ} 44^{\prime} \mathrm{E}, 6 \mathrm{~m}$ depth. 


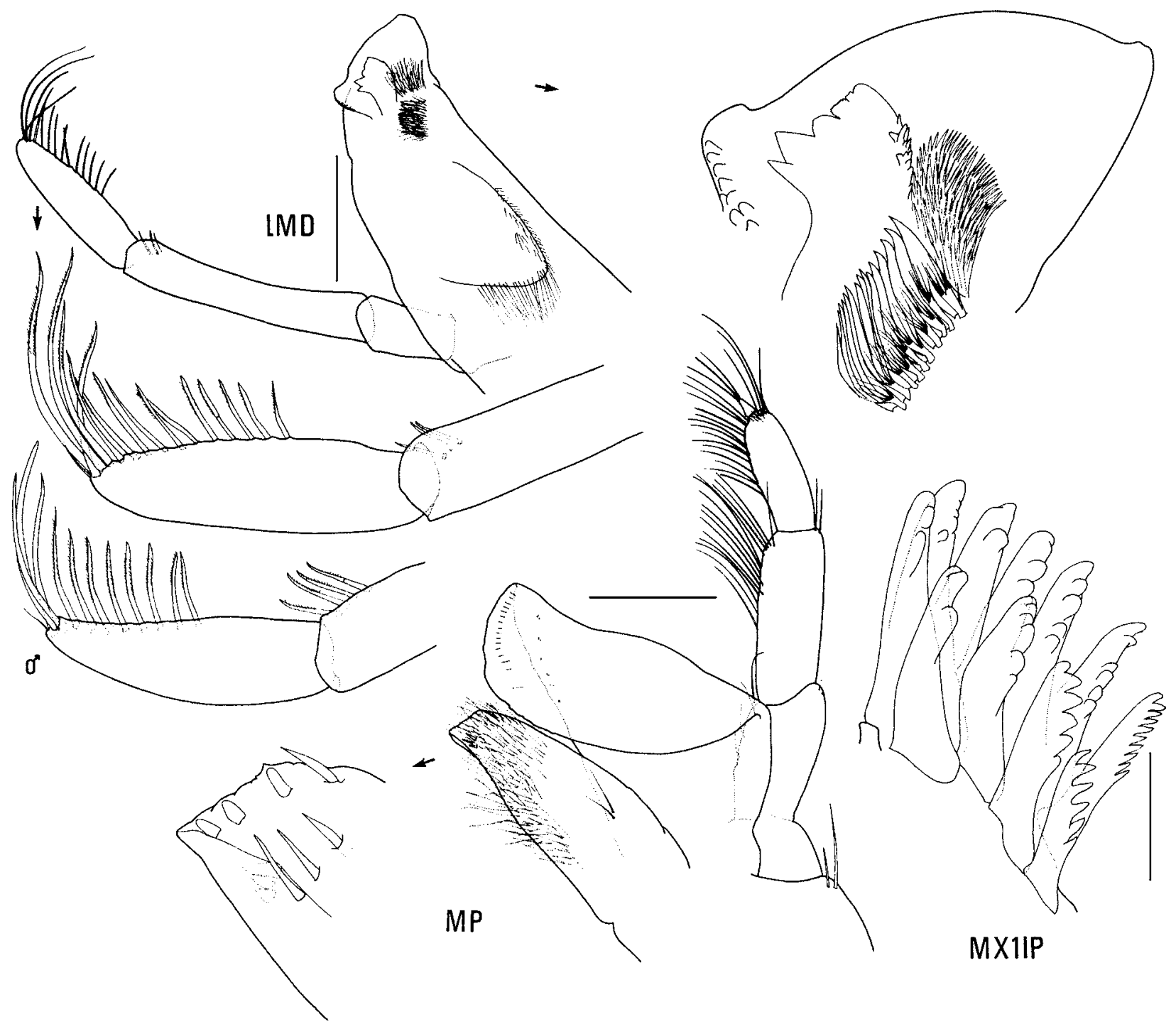

Figure 11. Amaryllis dianae n.sp., holotype female, $16.5 \mathrm{~mm}$, AM P37378; paratype immature male, $6.0 \mathrm{~mm}, \mathrm{AM}$ P37380; Fremantle, Western Australia. Scales represent $0.1 \mathrm{~mm}$.

Description. Based on holotype female, $16.5 \mathrm{~mm}$, AM P37378. Head much deeper than long, anterior margin with notch extended into a slit; rostrum absent; eye present, elongate, reniform. Antenna 1 peduncular article 1 not ballshaped proximally, distal margin with small medial spine; peduncular article 2 medium length; flagellum without callynophore, calceoli absent. Antenna 2 flagellum about as long as that of antenna 1, without calceoli. Mouthpart bundle subconical. Epistome/upper lip with moderate midanterior angle with small depression above angle (lateral view). Mandible lacinia mobilis a stemmed, distally-cusped blade; accessory setal row with intermediate setae; palp article 2 with 3 posterodistal setae, article 3 without A3seta. Maxilliped outer plate with distal margin smooth, medial margin without notch.

Gnathopod 1 carpus shorter than propodus; propodus, posterior margin without robust setae. Gnathopod 2 palm slightly acute, without lateral robust setae, 2-3 medial robust setae. Pereopods 3 and 4 merus and carpus without setal fringe. Pereopod 4 coxa with anterior and posterior margins subparallel, anteroventral corner rounded. Pereopods 5-7 with distal articles elongate, dactyls short and stocky. Pereopod 5 basis expanded posteriorly, rounded. Pereopod 7 basis subrectangular, posteroventral corner subquadrate, posteroventral margin straight.

Epimeron 3 posterior margin serrate, with notch well above rounded posteroventral corner. Uropod 1 peduncle dorsolateral margin with 17 robust setae; outer ramus without large spines between robust setae. Uropod 2 inner ramus slightly constricted. Uropod 3 rami lanceolate; without plumose setae; outer ramus 1-articulate. Telson moderately cleft (about 41\%).

Male (sexually dimorphic characters). Based on paratype male, $6.0 \mathrm{~mm}$, AM P37380 (probably not fully mature). Antenna 1 flagellum with callynophore. Antenna 2 flagellum with calceoli. Gnathopod 2 palm with 1 lateral robust seta, without medial robust setae.

Etymology. Named for Diana Jones, carcinologist at the Western Australian Museum, in appreciation of her generous help with collections of Western Australian amphipods.

Remarks. Amaryllis dianae is most similar to other Amaryllis species with a serrate posterior margin on 


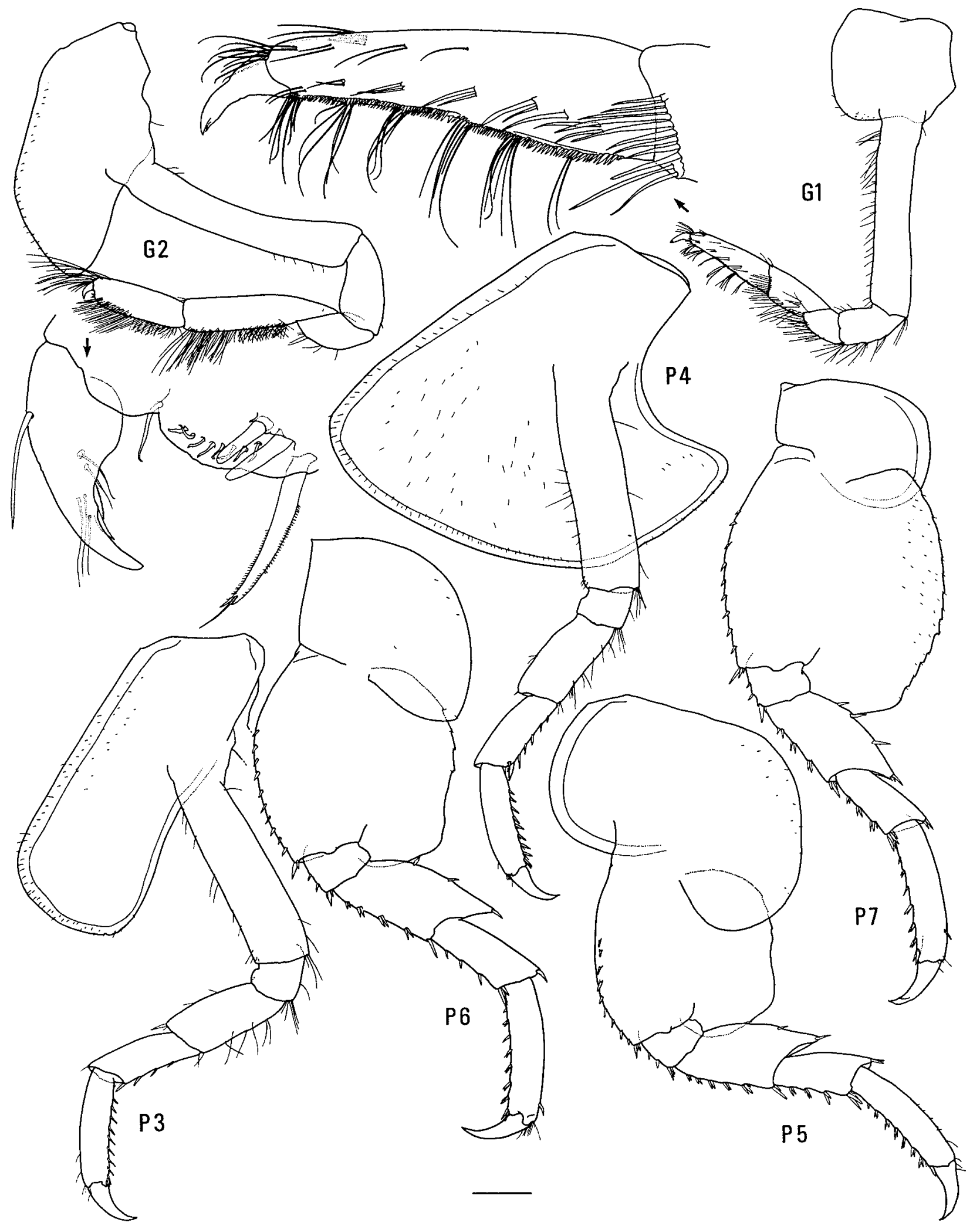

Figure 12. Amaryllis dianae n.sp., holotype female, 16.5 mm, AM P37378, Fremantle, Western Australia. Scales represent $0.5 \mathrm{~mm}$.

epimeron 3, such as A. carrascoi, A. croca, A. keablei and A. migo. Amaryllis dianae, A. carrascoi and A. migo all have a notch above the posteroventral corner of epimeron 3 ; in A. croca and A. keablei the serrations extend to the posteroventral corner. The posteroventral corner of pereopod 7 is rounded in A. migo, subquadrate in A. dianae and subquadrate with a distinct notch in A. carrascoi.

Distribution. Southern and southwestern Australia; 3-219 m depth. 


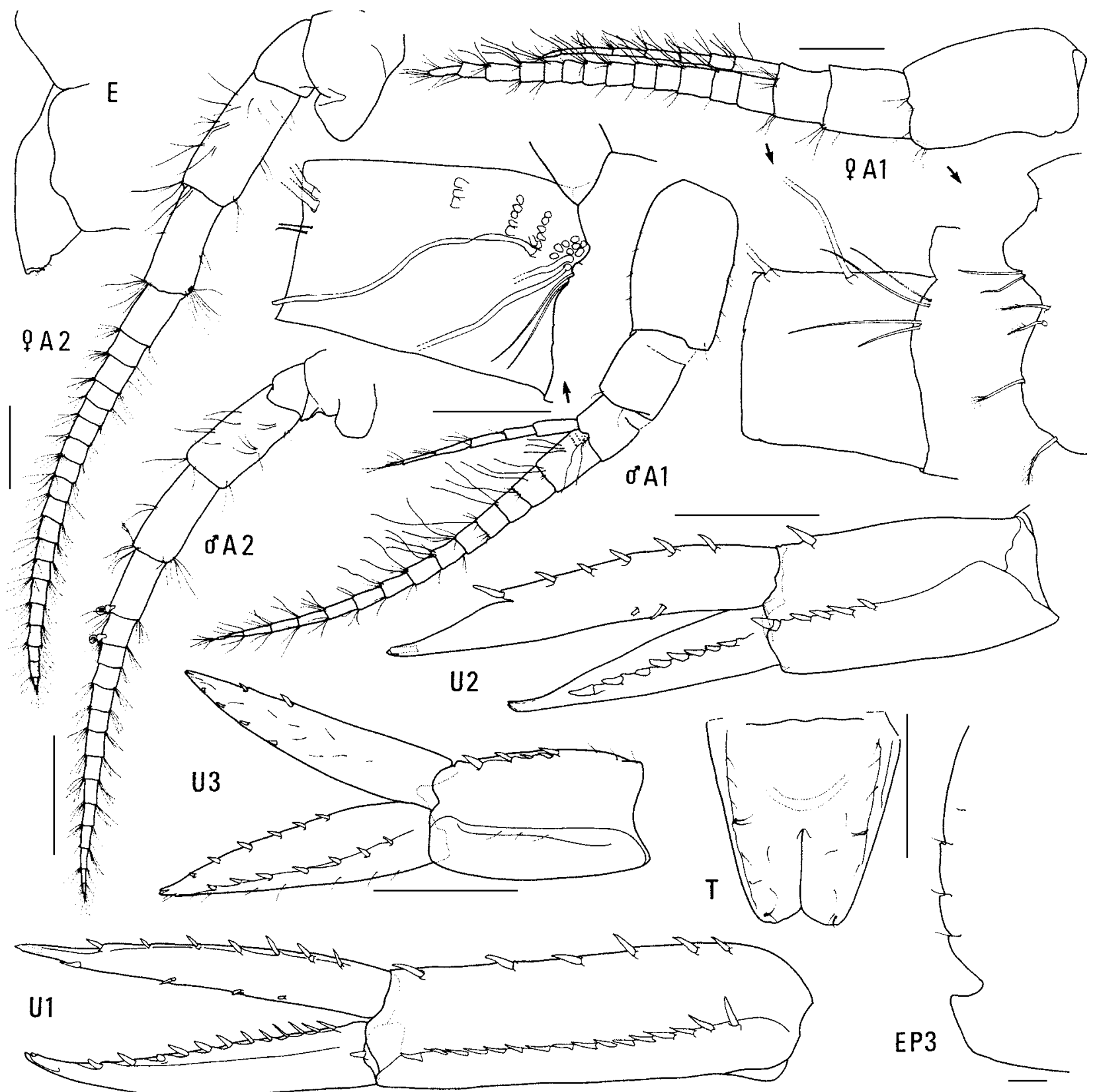

Figure 13. Amaryllis dianae n.sp., holotype female, $16.5 \mathrm{~mm}$, AM P37378; paratype immature male, $6.0 \mathrm{~mm}$, AM P37380; Fremantle, Western Australia. Scale for EP3 represents $0.1 \mathrm{~mm}$; remainder represent $0.5 \mathrm{~mm}$.

\section{Amaryllis kamata n.sp.}

Figs. 14-16

Amaryllis macrophthalma. Stebbing, 1910a: 569 (in part).

Type material. HOLOTYPE, female, $9.2 \mathrm{~mm}$, ovigerous (14 eggs), AM P37107; 1 PARATYPE, male, 6.2 mm, AM P37108; 44 PARATYPES, AM P37109; E of Long Reef, NSW, Australia, $33^{\circ} 46^{\prime} \mathrm{S} 151^{\circ} 43^{\prime} \mathrm{E}$, dredged, $176 \mathrm{~m}, 5$ December 1977, FRV Kapala, stn K77-23-01.

Additional material. NEW SOUTH WALES: 11 specimens, AM P2468, $6.5-10.5 \mathrm{~km}$ off Manning River, [approx. $31^{\circ} 57^{\prime} \mathrm{S} 152^{\circ} 46^{\prime} \mathrm{E}$ ], $40 \mathrm{~m}$, fine grey sand, trawl, 5 March 1898, HMCS Thetis, stn 28. 10 specimens, AM P37110, E of Newcastle, $32^{\circ} 53^{\prime} \mathrm{S} 152^{\circ} 35^{\prime} \mathrm{E}, 165 \mathrm{~m}, 15$ August 1985 , FRV Kapala, stn K85-12-23. 28 specimens, AM P59045, NE of Broken Bay, $33^{\circ} 22^{\prime} \mathrm{S} 152^{\circ} 04^{\prime} \mathrm{E}$ to $33^{\circ} 24^{\prime} \mathrm{S} 152^{\circ} 04^{\prime} \mathrm{E}, 181-112 \mathrm{~m}$, benthic trawl, 11 February 1986, FRV Kapala stn K86-01-04. 1 specimen, AM P37111,
$\mathrm{NE}$ of Broken Bay, $33^{\circ} 29^{\prime} \mathrm{S} 152^{\circ} 06^{\prime} \mathrm{E}$ to $33^{\circ} 30^{\prime} \mathrm{S} 152^{\circ} 04^{\prime} \mathrm{E}, 492-523 \mathrm{~m}$, trawl, 11 February 1986, FRV Kapala, stn K86-01-05. 2 specimens, AM P37395, SE of Broken Bay, $33^{\circ} 36^{\prime} \mathrm{S} 151^{\circ} 30^{\prime} \mathrm{E}$ to $33^{\circ} 37^{\prime} \mathrm{S} 151^{\circ} 29^{\prime} \mathrm{E}, 71-$ 75 m, 10 February 1986, FRV Kapala, stn K86-01-02. 1 specimen, P37112, NE of Long Reef Point, 33 ${ }^{\circ} 41^{\prime} \mathrm{S} 151^{\circ} 53^{\prime} \mathrm{E}, 366 \mathrm{~m}, 5$ December 1977, FRV Kapala, stn K77-23-03. 114 specimens, AM P37113, E of Long Reef Point, $33^{\circ} 43^{\prime} \mathrm{S} 151^{\circ} 46^{\prime} \mathrm{E}$ to $33^{\circ} 44^{\prime} \mathrm{S} 151^{\circ} 56^{\prime} \mathrm{E}, 174 \mathrm{~m}, 20$ December 1985, FRV Kapala, stn K85-21-08. 10 specimens, AM P37114, E of Bondi, 33 $52^{\prime} \mathrm{S} 151^{\circ} 23^{\prime} \mathrm{E}, 80 \mathrm{~m}, 11$ December 1980, FRV Kapala, stn K80-20-11. 2 specimens, AM P2469, 8-9.5 km off Coogee, [approx. $\left.33^{\circ} 57^{\prime} \mathrm{S} 151^{\circ} 21.5^{\prime} \mathrm{E}\right], 90-91 \mathrm{~m}$, fine sand, trawl, E.R. Waite, 15 March 1898, HMCS Thetis, stn 44. 1 specimen, AM P58759, Cape Banks, $34^{\circ} 00^{\prime} \mathrm{S} 151^{\circ} 16^{\prime} \mathrm{E}, 45-50 \mathrm{~m}$, The Ecology Lab, November 1990, stn S2T3. 2 specimens, AM P5427, 4-6 km E of Botany Bay, [approx. 3401'S $\left.151^{\circ} 19^{\prime} \mathrm{E}\right], 60-102 \mathrm{~m}$, trawl, F.A. McNeill \& A. Livingstone, August 1921, ST Goonambee. 26 specimens, AM P2466, 2-3 km off Port Hacking, [approx. $34^{\circ} 03.5^{\prime} \mathrm{S} 151^{\circ} 12.5^{\prime} \mathrm{E}$ ], 40-69 m, sandy, trawl, E.R. Waite, 10 March 1898, HMCS Thetis, stn 35. 34 specimens, AM P2464 and 10 specimens, AM P2465, 3-4 km off Botany Bay, [approx. 34은 $\left.151^{\circ} 15^{\prime} \mathrm{E}\right], 91-95 \mathrm{~m}$, mud, trawl, E.R. Waite, 11 March 1898, HMCS 


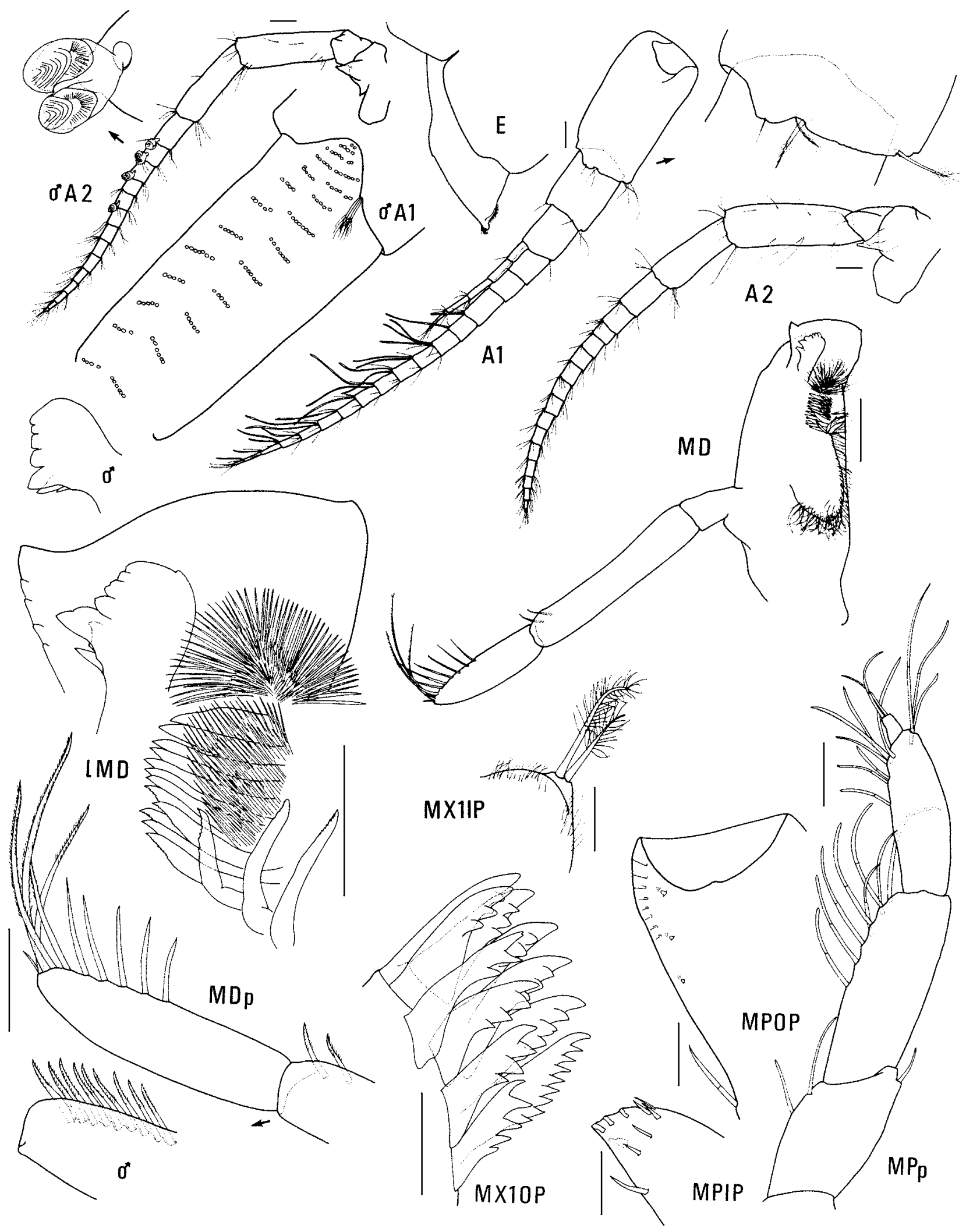

Figure 14. Amaryllis kamata n.sp., holotype female, $9.2 \mathrm{~mm}$, AM P37107; paratype male, $6.2 \mathrm{~mm}$, AM P37108; east of Long Reef, NSW. Scales for A1, 2 represent $0.1 \mathrm{~mm}$; remainder represent $0.05 \mathrm{~mm}$.

Thetis, stn 37. 64 specimens, AM P2467, 5.5-6.5 km off Wata Mooli [now Wattamolla], [approx. $34^{\circ} 10^{\prime} \mathrm{S} 151^{\circ} 11^{\prime} \mathrm{E}$ ], 108-99 m, mud, trawl, E.R. Waite, 22 March 1898, HMCS Thetis, stn 57. 1 specimen, AM $\mathrm{P} 37115$, NE of Montague Island, $35^{\circ} 58^{\prime} \mathrm{S} 150^{\circ} 30^{\prime} \mathrm{E}$ to $36^{\circ} 03^{\prime} \mathrm{S} 150^{\circ} 27^{\prime} \mathrm{E}$, 384 m, 21 November 1979, FRV Kapala, stn K79-18-07. BASS STRAIT: 7 specimens, AM P58116, eastern Bass Strait, $38^{\circ} 56.5^{\prime} \mathrm{S} 148^{\circ} 19.3^{\prime} \mathrm{E}, 8^{\circ}$
85 m, benthic sled, P.B. Berents, 27 August 1994, FRV Southern Surveyor, stn 05/94/59. 6 specimens, AM P58117, eastern Bass Strait, 38 $59.1^{\circ} \mathrm{S}$ $148^{\circ} 31.6^{\prime} \mathrm{E}, 125 \mathrm{~m}$, benthic sled, P.B. Berents, 27 August 1994, FRV Southern Surveyor, stn 05/94/60. TASMANIA: 1 specimen, AM E6526, Tasmanian coast, FIS Endeavour Collection. 


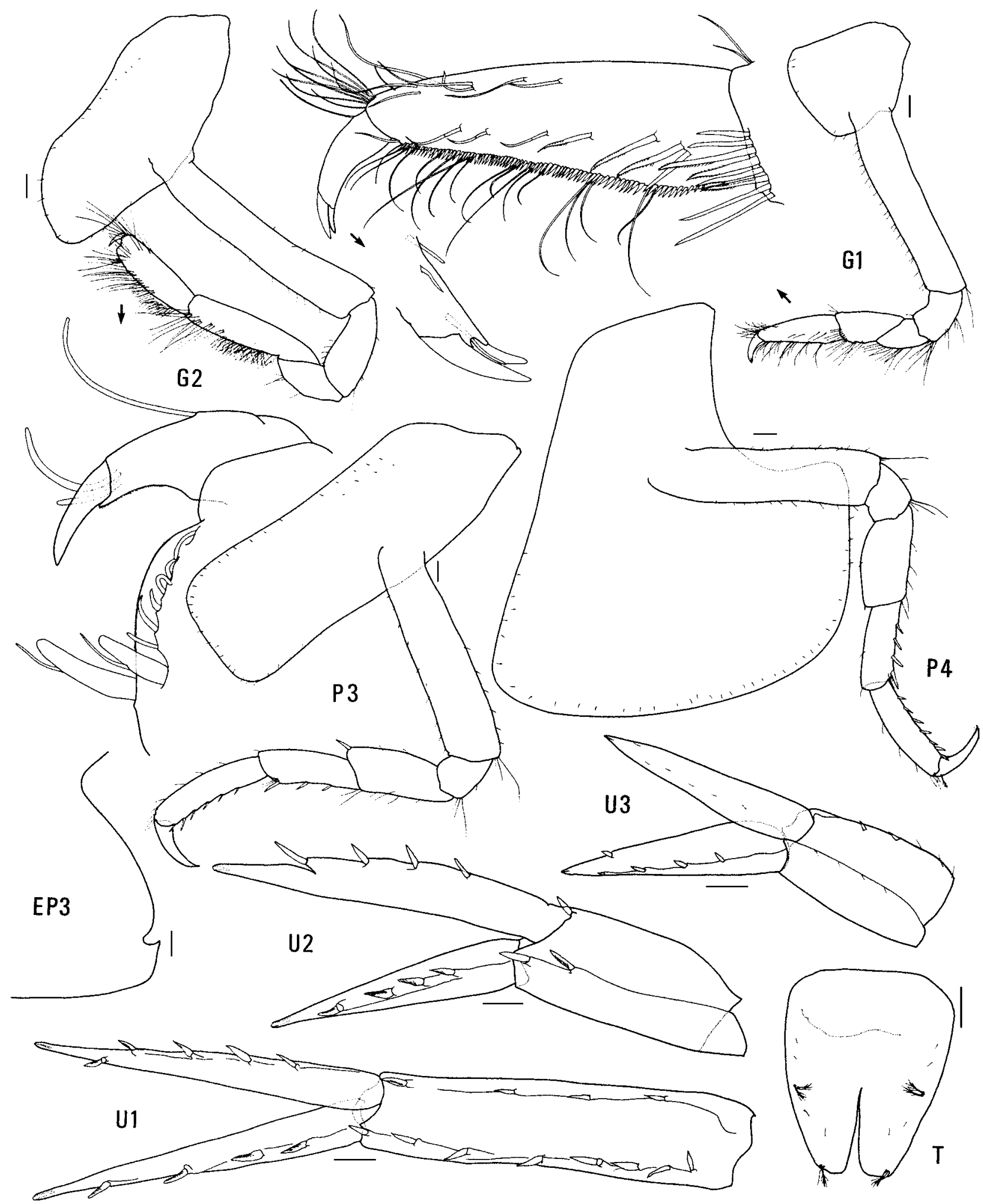

Figure 15. Amaryllis kamata n.sp., holotype female, $9.2 \mathrm{~mm}$, AM P37107, east of Long Reef, NSW. Scales represent $0.1 \mathrm{~mm}$.

Type locality. East of Long Reef, NSW, Australia, $33^{\circ} 46^{\prime} \mathrm{S}$ $151^{\circ} 43^{\prime} \mathrm{E}, 176 \mathrm{~m}$ depth.

Description. Based on holotype female, $9.2 \mathrm{~mm}$, AM P37107. Head much deeper than long, anterior margin with notch extended into a slit; rostrum absent; eye present, elongate, reniform. Antenna 1 peduncular article 1 not ballshaped proximally, distal margin with small medial spine; peduncular article 2 medium length; flagellum without callynophore, calceoli absent. Antenna 2 flagellum about as long as that of antenna 1, without calceoli. Mouthpart bundle subconical. Epistome/upper lip with moderate midanterior angle with small depression above angle (lateral view). Mandible lacinia mobilis a stemmed, distally-cusped blade; accessory setal row with intermediate setae; palp article 2 with 2 posterodistal setae, article 3 without A3- 


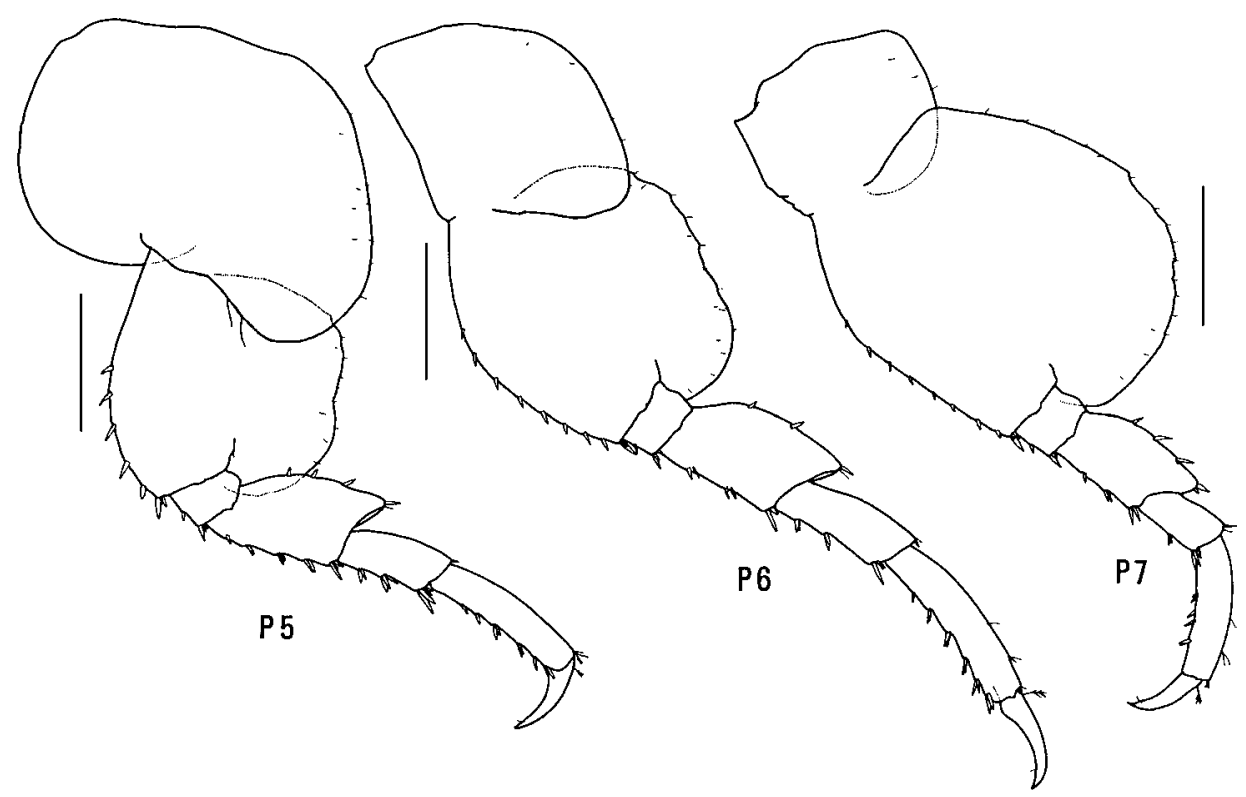

Figure 16. Amaryllis kamata n.sp., holotype female, $9.2 \mathrm{~mm}$, AM P37107, east of Long Reef, NSW. Scales represent $0.5 \mathrm{~mm}$.

seta. Maxilliped outer plate with distal margin smooth, medial margin without notch.

Gnathopod 1 carpus subequal in length to propodus $(0.9 \times)$; propodus, posterior margin without robust setae. Gnathopod 2 palm slightly acute, with 1 lateral robust seta, 1 medial robust seta. Pereopods 3 and 4 merus and carpus without setal fringe. Pereopod 4 coxa with anterior and posterior margins subparallel, anteroventral corner rounded. Pereopods 5-7 with distal articles elongate, dactyls short and stocky. Pereopod 5 basis expanded posteriorly, rounded. Pereopod 7 basis rounded posteriorly, posteroventral corner rounded, posteroventral margin straight.

Epimeron 3 posterior margin smooth, with notch well above rounded posteroventral corner. Uropod 1 peduncle dorsolateral margin with 8 robust setae; outer ramus without large spines between robust setae. Uropod 2 inner ramus slightly constricted. Uropod 3 rami lanceolate; without plumose setae; outer ramus 1-articulate. Telson moderately cleft (about 43\%).

Male (sexually dimorphic characters). Based on paratype male, $6.2 \mathrm{~mm}$, AM P37108. Antenna 1 flagellum with callynophore, with calceoli. Antenna 2 flagellum with calceoli. Mandible palp article 2 with 9 posterodistal setae. Gnathopod 2 palm with no lateral robust setae and 1 medial robust seta.

Etymology. The specific name is contrived and has no special meaning.

Remarks. Amaryllis kamata belongs to the group of species with a smooth posterior margin on epimeron 3 and a rounded posteroventral corner on the basis of pereopod 7. Within this group it is most similar to A. brevicornis but differs in the shape of the epistome/upper lip and the length of the gnathopod 1 carpus.

Habitat. Amaryllis kamata is known from sand and mud bottoms off the New South Wales coast. It is the deepestoccurring species (50 to $525 \mathrm{~m}$ depth) of Amaryllis known from Australian waters.

Distribution. Southeastern Australia; 40-523 m depth.

\section{Amaryllis keablei n.sp.}

Figs. 17-19

Amaryllis macrophthalma.-Chilton, 1921: 55 (in part).-J.L. Barnard, 1972: 262, fig. 156 (in part, figs. of female "n"). Amaryllis sp. 1.-Hutchings et al., 1989: 362.

Type material. Holotype, female, $20.5 \mathrm{~mm}$, ovigerous (23 eggs), NMV J3765, 23 km east of Cape Rochon, Three Hummock Island, central Bass Strait, Tasmania, Australia, $40^{\circ} 22.2^{\prime} \mathrm{S} 145^{\circ} 17^{\prime} \mathrm{E}, 40 \mathrm{~m}$, sand, epibenthic sled, M. Gomon \& G.C.B. Poore, 3 November 1980, FRV Sarda, stn BSS 112. 3 PARATYPES, female, NMV J13946; 3 PARATYPES, female, AM P37100; 9 km SSW of Cape Adansan, Three Hummock Island, central Bass Strait, Tasmania, Australia, 40³0.9'S $144^{\circ} 56^{\prime} \mathrm{E}, 27 \mathrm{~m}$, very coarse sand, epibenthic sled, M. Gomon \& G.C.B. Poore, 2 November 1980, FRV Sarda, stn BSS 109.

Additional material. NEW SOUTH WALES: 1 specimen, NMV J13947, Port Stephens, [approx. $32^{\circ} 42^{\prime}$ S $152^{\circ} 06^{\prime} E$ ], O.A. Sayce Collection. 2 specimens, AM P37101, Port Jackson. 1 specimen, AM P37180, Port Jackson [was part of Amaryllis brevicornis syntype series, AM G5417]. 2 specimens, AM P36700, Quarantine Bay side of Murrumbulga Point, Twofold Bay, $37^{\circ} 04.7$ 'S $149^{\circ} 53.1^{\prime} \mathrm{E}, 3 \mathrm{~m}$, subtidal breakwater wall, S. Keable \& P. Hutchings, 17 September 1985, stn FIRTA Q9-Q11. 1 male (illustrated), AM P37102, Munganno Point, Twofold Bay, 3706.2'S $149^{\circ} 55.7^{\prime} \mathrm{E}, 7 \mathrm{~m}$, sublittoral rock platform, P. Hutchings, 10 October 1984 stn FIRTA M3. VICTORIA: 2 females, NMV J13948, Crawfish Rock, Western Port, 14 m. 4 specimens, NMV J13949, Western Port. 2 females, NMV J3789, Western Port, 28 April 1968, stn D/1/11. 1 specimen, NMV J13950, Crib Point, Western Port, 38 ${ }^{\circ} 20.83$ 'S $145^{\circ} 13.49^{\prime} \mathrm{E}, 13 \mathrm{~m}$, sandy gravel, 23 March 1965, stn CPBS 32N. 3 females, NMV J3241, Port Phillip Bay, stn 25. 1 ovigerous female, NMV J13951, Port Phillip Bay, stn 26. 1 female, NMV J3262, mouth of Werribee River to centre of Bay, Port Phillip Bay, W. Williams, 20 March 1963, stn 25, area 9 to 19.1 specimen, AM P38945, Ricketts Point, Port Phillip Bay, [approx. 37058'S $144^{\circ} 54^{\prime} \mathrm{E}$ ], 2 m, rock shelf, N. Coleman, 21 June 1979, AMPI Crust No. 899. BASS STRAIT: 1 specimen, NMV J13952, $33 \mathrm{~km} \mathrm{~S}$ of Deal Island, Tasmania, Central Bass Strait, 39 47.3'S 147 $19.3^{\prime} \mathrm{E}, 60 \mathrm{~m}$, muddy sand, epibenthic sled, R. Wilson, 14 November 1981, RV Tangaroa, stn BSS-161. TASMANIA: 1 specimen, AM P37103, Derwent Estuary, D'Entrecasteaux Channel, March 1940, Tasmanian Biological Survey, Fisheries Department, stn OL81. 1 specimen, AM P5913, Tasmanian Coast, FIS Endeavour. South AUSTRALIA: 1 specimen, SAMA C5980, Dangerous 


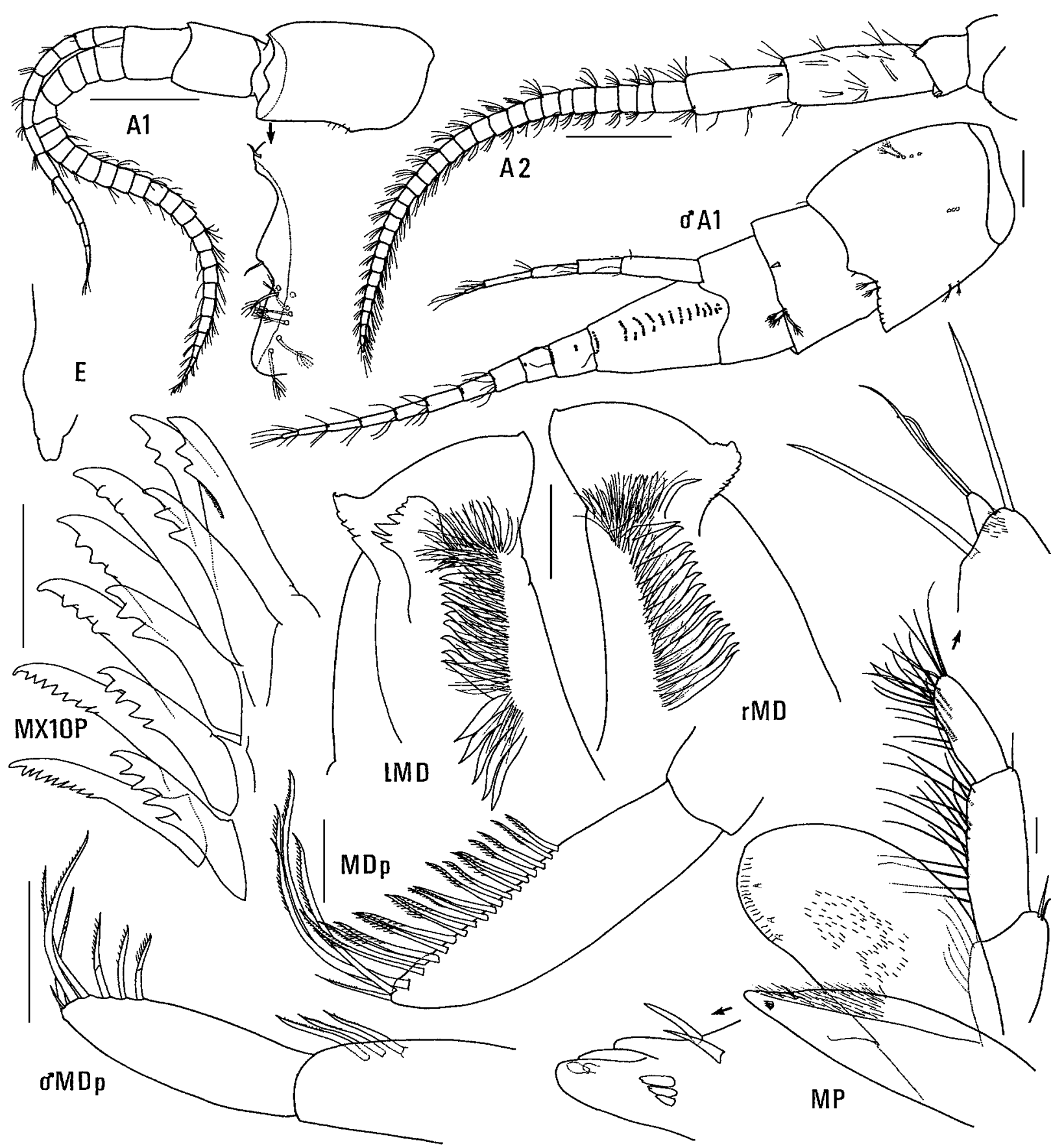

Figure 17. Amaryllis keablei n.sp., holotype female, 20.5 mm, NMV J3765, Bass Strait; male, 5.8 mm, AM P37102; Twofold Bay, NSW. Scales for A1, 2 represent $0.5 \mathrm{~mm}$; remainder represent $0.1 \mathrm{~mm}$.

Reef, Spencer Gulf, [approx. 34 $49^{\prime}$ S $136^{\circ} 12^{\prime} E$ ], dredged, K. Sheard. 1 specimen, NMV J13953, The Hotspot Reef, W of Flinders Island, $33^{\circ} 40.8^{\prime} \mathrm{S} 134^{\circ} 22.5^{\prime} \mathrm{E}, 21 \mathrm{~m}$, erect hard bryozoan on vertical rock face, G.C.B. Poore, 20 April 1985, stn NMV SA-71. 2 females, AM P37104, Marino, Gulf St Vincent, [approx. 3503'S $138^{\circ} 30^{\prime} E$ ], W.H. Baker, 1910. 1 specimen, AM P37105, Port Adelaide, Gulf St Vincent, [approx. 3450'S $138^{\circ} 30^{\prime}$ E], H.M. Hale, 1924. 1 female, AM P37106, $19 \mathrm{~km}$ from Mount Young toward Wallaroo, Spencer Gulf, [approx. $\left.33^{\circ} 18^{\prime} \mathrm{S} 137^{\circ} 31^{\prime} \mathrm{E}\right], 18$ m, K. Sheard, 8 March 1938, FL Whyalla.

Type locality. $23 \mathrm{~km}$ east of Cape Rochon, Three Hummock Island, central Bass Strait, Tasmania, Australia, 40 22.2'S $145^{\circ} 17^{\prime} \mathrm{E}, 40 \mathrm{~m}$ depth.

Description. Based on holotype female, $20.5 \mathrm{~mm}$, NMV J3765. Head much deeper than long, anterior margin with notch extended into a slit; rostrum absent; eye present, elongate, reniform. Antenna 1 peduncular article 1 not ballshaped proximally, distal margin with small medial spine; peduncular article 2 medium length; flagellum without callynophore, calceoli absent. Antenna 2 flagellum about as long as that of antenna 1, without calceoli. Mouthpart bundle subconical. Epistome/upper lip with strong midanterior angle (lateral view). Mandible lacinia mobilis a stemmed, distally-cusped blade; accessory setal row with intermediate setae; palp article 2 without posterodistal setae, article 3 without A3-seta. Maxilliped outer plate with distal margin smooth, medial margin deeply notched.

Gnathopod 1 carpus subequal in length to propodus; propodus, posterior margin without robust setae. Gnathopod 


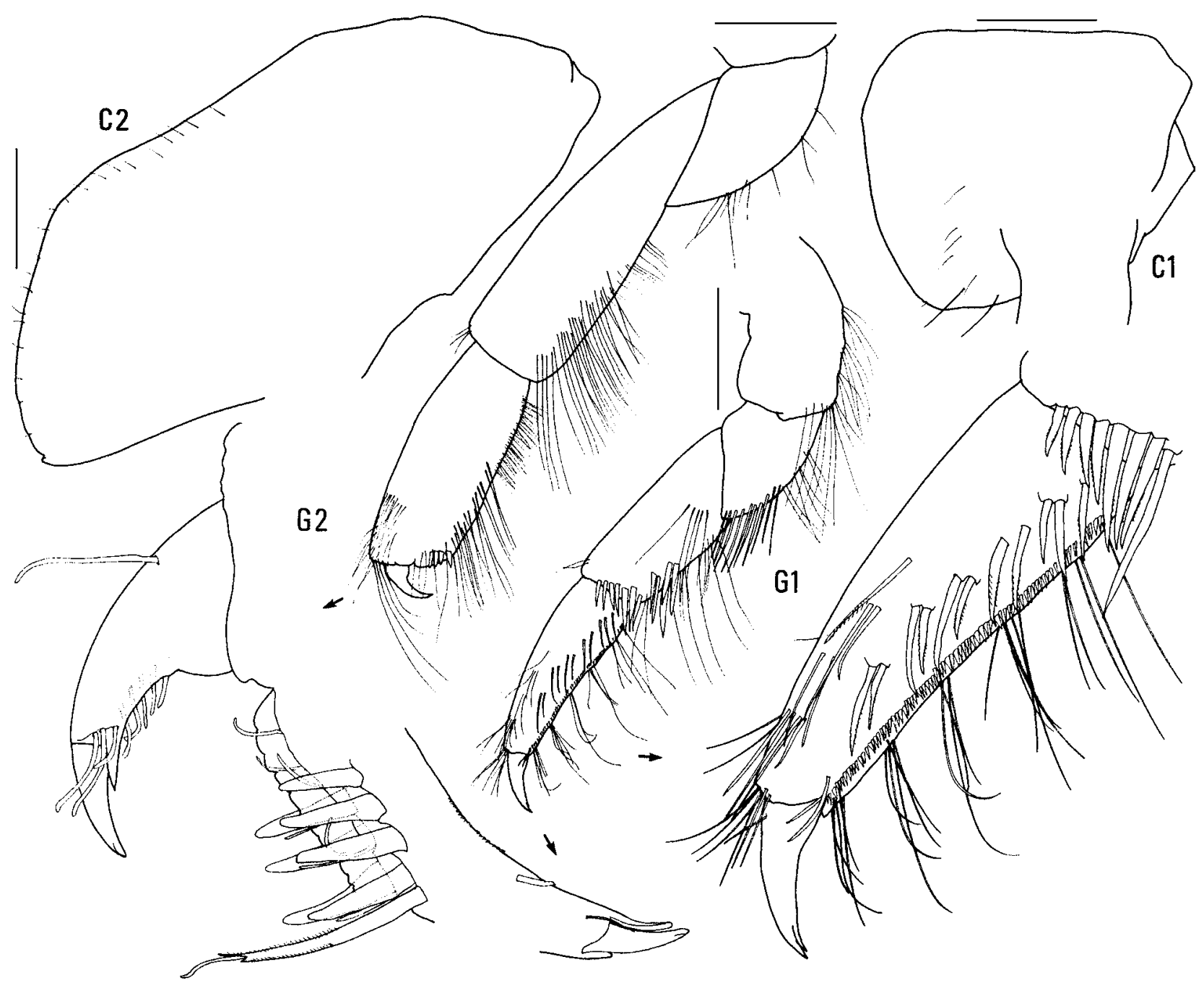

Figure 18. Amaryllis keablei n.sp., holotype female, 20.5 mm, NMV J3765, Bass Strait. Scales represent 0.5 mm.

2 palm slightly acute, with 3-4 lateral robust setae, 4 medial robust setae. Pereopods 3 and 4 merus and carpus without setal fringe. Pereopod 4 coxa with anterior and posterior margins subparallel, anteroventral corner rounded. Pereopods 5-7 with distal articles elongate, dactyls short and stocky. Pereopod 5 basis expanded posteriorly, rounded. Pereopod 7 basis subrectangular, posteroventral corner subquadrate, posteroventral margin straight.

Epimeron 3 posterior margin serrate, without notch. Uropod 1 peduncle dorsolateral margin with 17 robust setae; outer ramus without large spines between robust setae. Uropod 2 inner ramus slightly constricted. Uropod 3 rami lanceolate; without plumose setae; outer ramus 1-articulate. Telson moderately cleft (about 37\%).

Male (sexually dimorphic characters). Based on male, 5.8 mm, AM P37102 (probably not fully mature). Antenna 1 flagellum with callynophore. Antenna 2 flagellum with calceoli. Mandible palp article 2 with 3 posterodistal setae.

Etymology. Named for Stephen Keable in recognition and appreciation of his meticulous work in producing illustrations of amphipods.

Remarks. Amaryllis keablei is similar to other Amaryllis species with a serrate posterior margin on epimeron 3 , such as $A$. carrascoi, $A$. croca, $A$. dianae and $A$. migo. It is most similar to $A$. croca in that the serrations extend to the posteroventral corner. Amaryllis keablei differs from A. croca in having a strong mid-anterior angle on the epistome/ upper lip, a small medial spine on pedunclar article 1 of antenna 1, no posterodistal setae on mandibular palp article 2 of the female and 3 to 4 lateral robust setae on the palm of female gnathopod 2.

Distribution. Southeastern and southern Australia, from Port Stephens on the east coast to the South Australian Gulfs; 2-60 m depth.

\section{Amaryllis macrophthalma Haswell}

Figs. 20-22

Amaryllis macrophthalmus Haswell, 1879: 253, pl. 8 fig. 3.Haswell, 1882: 227.-Della Valle, 1893: 781 (in part).-J.L. Barnard, 1974: 140.-Barnard \& Karaman, 1991: 461.Springthorpe \& Lowry, 1994: 129.

Amaryllis macrophthalma.-Stebbing, 1906: 24 (in part).-Chilton, 1921: 55 (in part).-Guiler, 1952: 28.-J.L. Barnard, 1958: 88.J.L. Barnard, 1972: 262, figs. 156-158 (in part).-Poore et al., 1975: 34, 67 (in part).

not Glycerina affinis Chilton, 1885: 1036, pl. 47 fig. 1.-Della Valle, 1893: 849. 


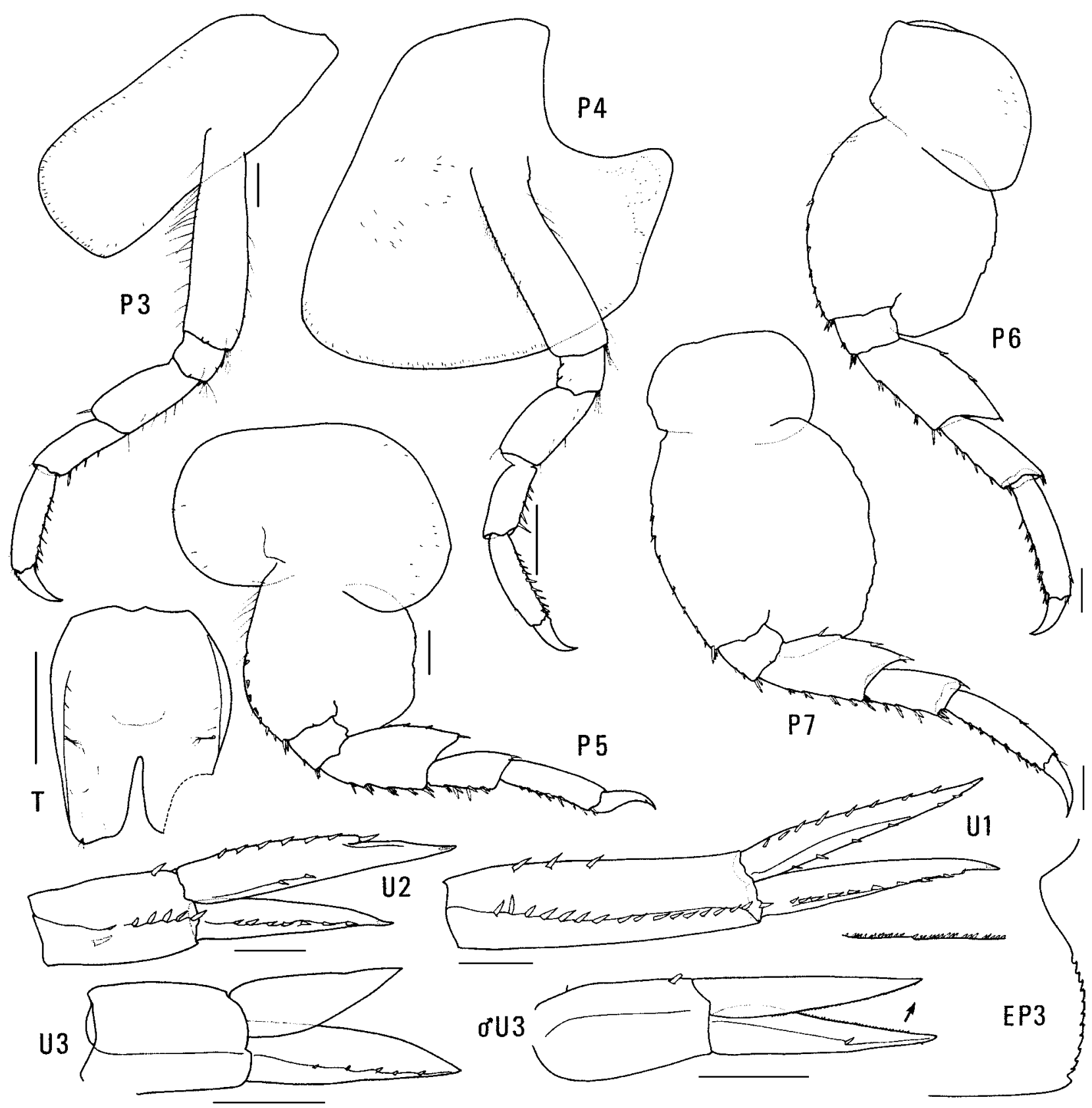

Figure 19. Amaryllis keablei n.sp., holotype female, 20.5 mm, NMV J3765, Bass Strait. Scales represent $0.5 \mathrm{~mm}$.

possibly Amaryllis macrophthalma.-Hale, 1929: 208.-Sheard, 1937: 18 (list).-Sergeev et al., 1988: 102 (table 1).

not Amaryllis macrophthalmus.-Stebbing, 1888: 706, pl. 29.-

Thomson, 1902: 463.-Hutton, 1904: 258.-Chilton, 1906: 267.-

Stebbing, 1908: 67.-Stebbing, 1910b: 448.

not Amaryllis macrophthalma.-Walker, 1909: 327.-Stebbing,

1910a: 569, 633 (= A. kamata and B. endota).-Chilton, 1912:

463.-K.H.Barnard, 1916: 114.-Schellenberg, 1926: 243.-

Schellenberg, 1931: 10.-K.H. Barnard, 1932: 34.-Pirlot, 1933:

122.-K.H.Barnard, 1937: 141.-Pirlot, 1939: 73.-K.H. Barnard, 1940: 514 (list).-Grindley \& Kensley, 1966: 8.-Day et al., 1970: 50.-Griffiths, 1973: 292.-Griffiths, 1974a: 199.Griffiths, 1974b: 247.-Griffiths, 1974c: 308.-Griffiths, 1975: 144.-Griffiths, 1976: 56, fig. 31A, B.-Lowry \& Bullock, 1976: 83.-Griffiths, 1977: 107.-Kensley \& Penrith, 1977: 189 (list).Ledoyer, 1978: 304.-Ledoyer, 1979: 112, fig. 71.-Ledoyer, 1986:

718, fig. 275.-Alonso, 1987: 2, figs. 1-15.-Gonzalez, 1991: 58. not Amaryllis ?macrophthalma.-Lyons \& Myers, 1991: 614, fig. 13.
Type material. NEOTYPE, female, $21.5 \mathrm{~mm}$, ovigerous (14 eggs), AM P37214, Derwent Estuary, D’Entrecasteaux Channel, Tasmania, Australia, [approx. 42 $54^{\prime} \mathrm{S} 147^{\circ} 23^{\prime} \mathrm{E}$ ], Fisheries Department, March 1940, Tasmanian Biological Survey, Station OL 81.

Haswell (1879) did not designate type material for $A$. macrophthalma, but he indicated that material examined by him was held in the Macleay Museum, University of Sydney. This material is now either lost or no longer exists (see J.L. Barnard, 1974:139-140 and Springthorpe \& Lowry, 1994: 129, for further discussion of Haswell's material). We are establishing a neotype for two reasons: Amaryllis macrophthalma is the type species of the genus (selected by Pirlot, 1933) and as such must be clearly and adequately established; we now recognize many species in the genus Amaryllis and a neotype is needed to clearly 


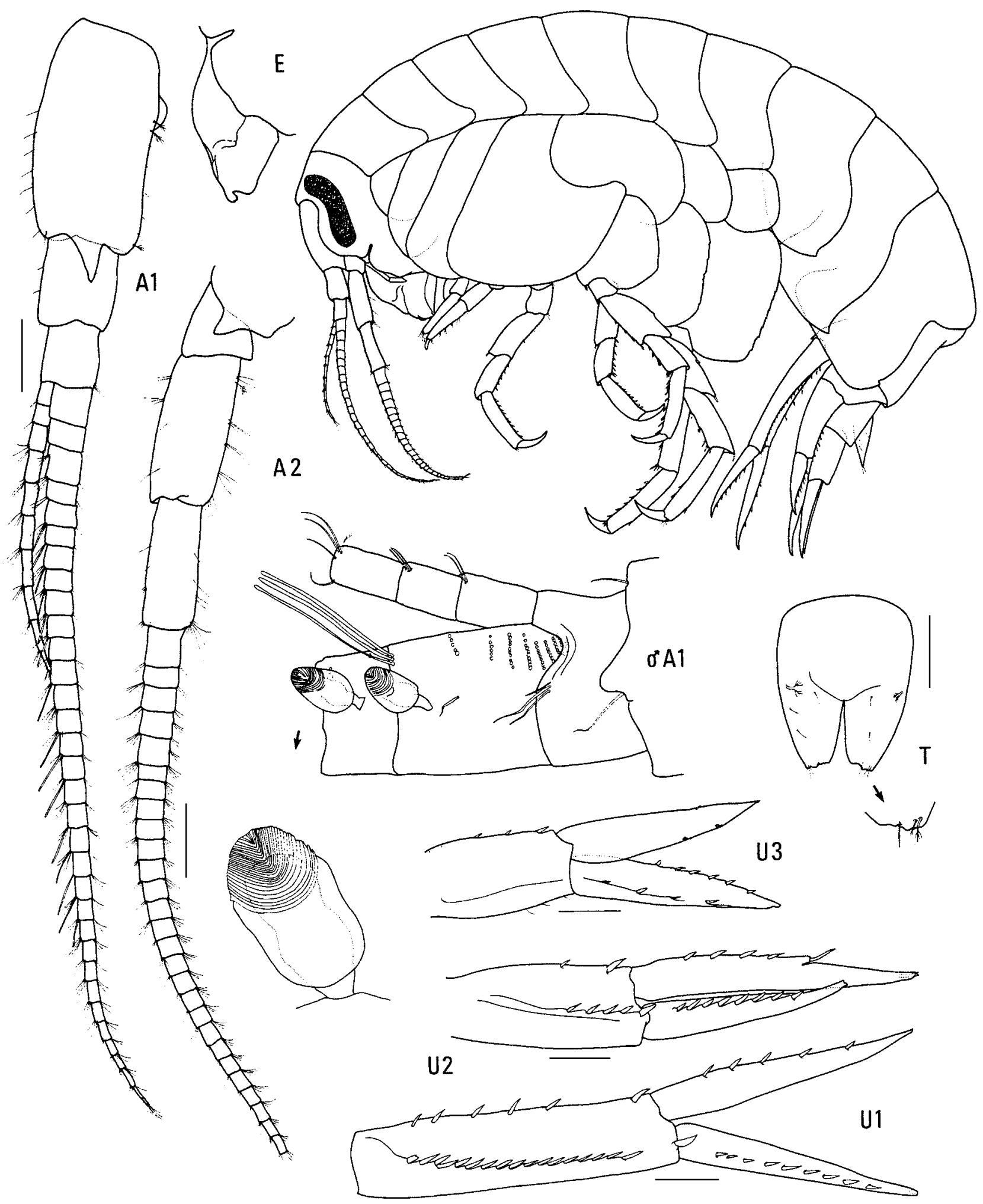

Figure 20. Amaryllis macrophthalma Haswell, neotype female, 21.4 mm, AM P37214; male, 17.8 m, AM P37215; Derwent Estuary, Tasmania. Scales represent $0.5 \mathrm{~mm}$.

distinguish A. macrophthalma from several closely related species.

The original locality for A. macrophthalma was given as "Tasmania". There is no extant material of A. macrophthalma from Tasmania in the Macleay Museum or the Australian Museum which could have been examined by
Haswell. We have examined material from Tasmania which includes at least three species. From Haswell's rather poor description and figures two characters, when taken together, are diagnostic: the square (rather than rounded) posterior margin of the basis of pereopod 7 (pl. 8 fig. 3) and the large size of the species ("nine-and-a-half lines" or approx. 20 


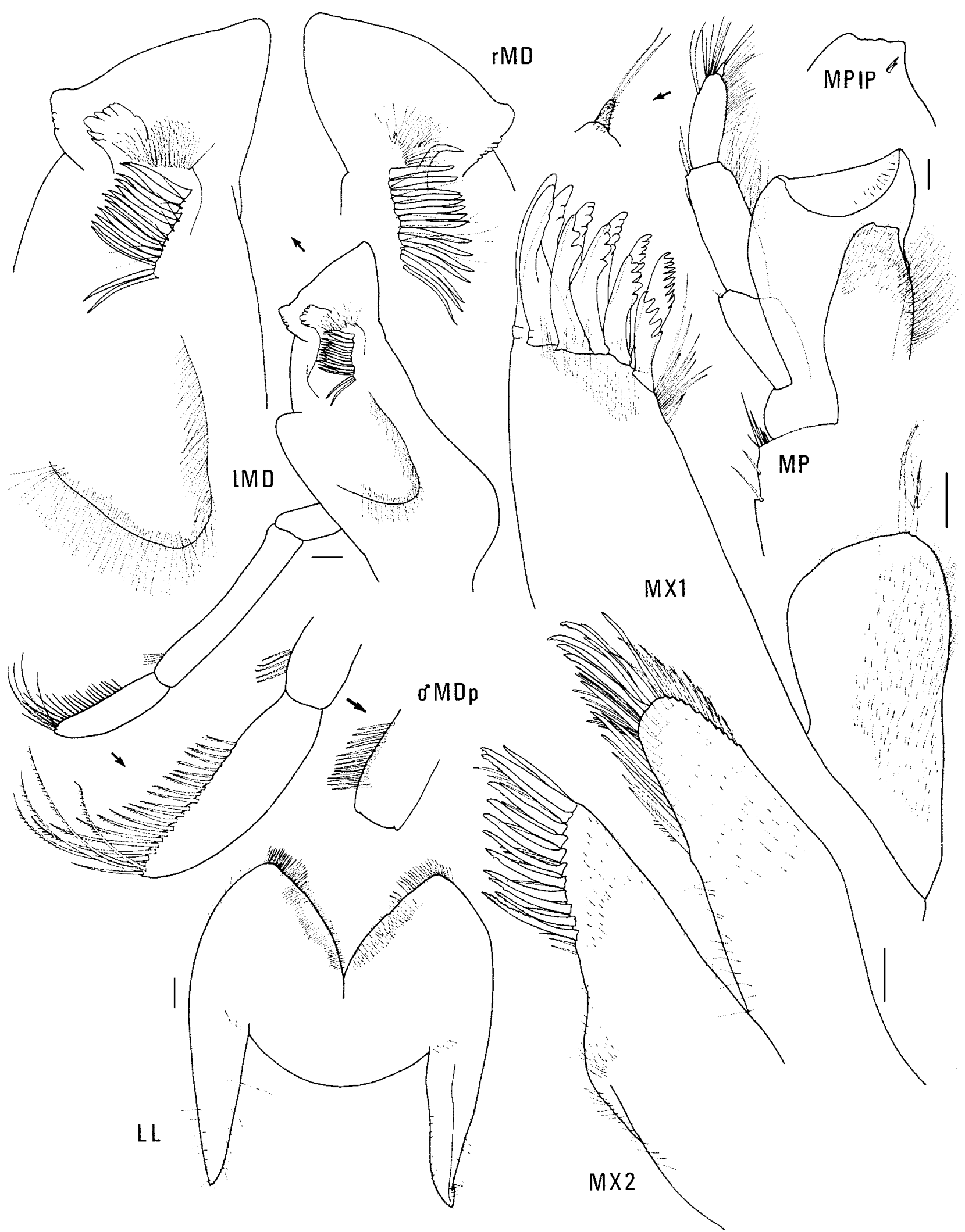

Figure 21. Amaryllis macrophthalma Haswell, neotype female, 21.4 mm, AM P37214; male, 17.8 m, AM P37215; Derwent Estuary, Tasmania. Scales represent $0.1 \mathrm{~mm}$.

$\mathrm{mm})$. These characters are the basis for our neotype selection.

Additional material. New SOUTH WALES: 3 females and 1 immature male, AM P37211, Port Jackson, [approx. 33 $52^{\prime} \mathrm{S} 151^{\circ} 13^{\prime} \mathrm{E}$ ], [from AM Old Collection]. 1 female, AM P18203, 8 km E of Green Cape, [approx. $\left.37^{\circ} 16^{\prime} \mathrm{S} 150^{\circ} 08^{\prime} \mathrm{E}\right], 82 \mathrm{~m}, \mathrm{~K}$. Moller, May 1930. 3 females, AM P18332,
19-35 km NE of Green Cape, [approx. $\left.37^{\circ} 00^{\prime} \mathrm{S} 150^{\circ} 08^{\prime} \mathrm{E}\right], 71-84 \mathrm{~m}$, A.A. Livingstone \& H.O. Fletcher, June 1924, Goonambee. Victoria: 1 female, AM P18268, SW of Point Hicks, 37 $55^{\prime} \mathrm{S} 149^{\circ} 00^{\prime} \mathrm{E}, 77 \mathrm{~m}$, R.J. MacIntyre, 20 June 1962, HMAS Gascoyne, stn G2/61/6. 13 specimens, NMV J11545, Bennison Channel, Corner Inlet, $38^{\circ} 49^{\prime} \mathrm{S} 146^{\circ} 23^{\prime} \mathrm{E}, 6 \mathrm{~m}$, sand and shell grit, airlift, G.J. Morgan, 23 November 1983, stn CIN 28E. 2 specimens, NMV J3793, Crawfish Rock, Western Port, 14 m. 34 


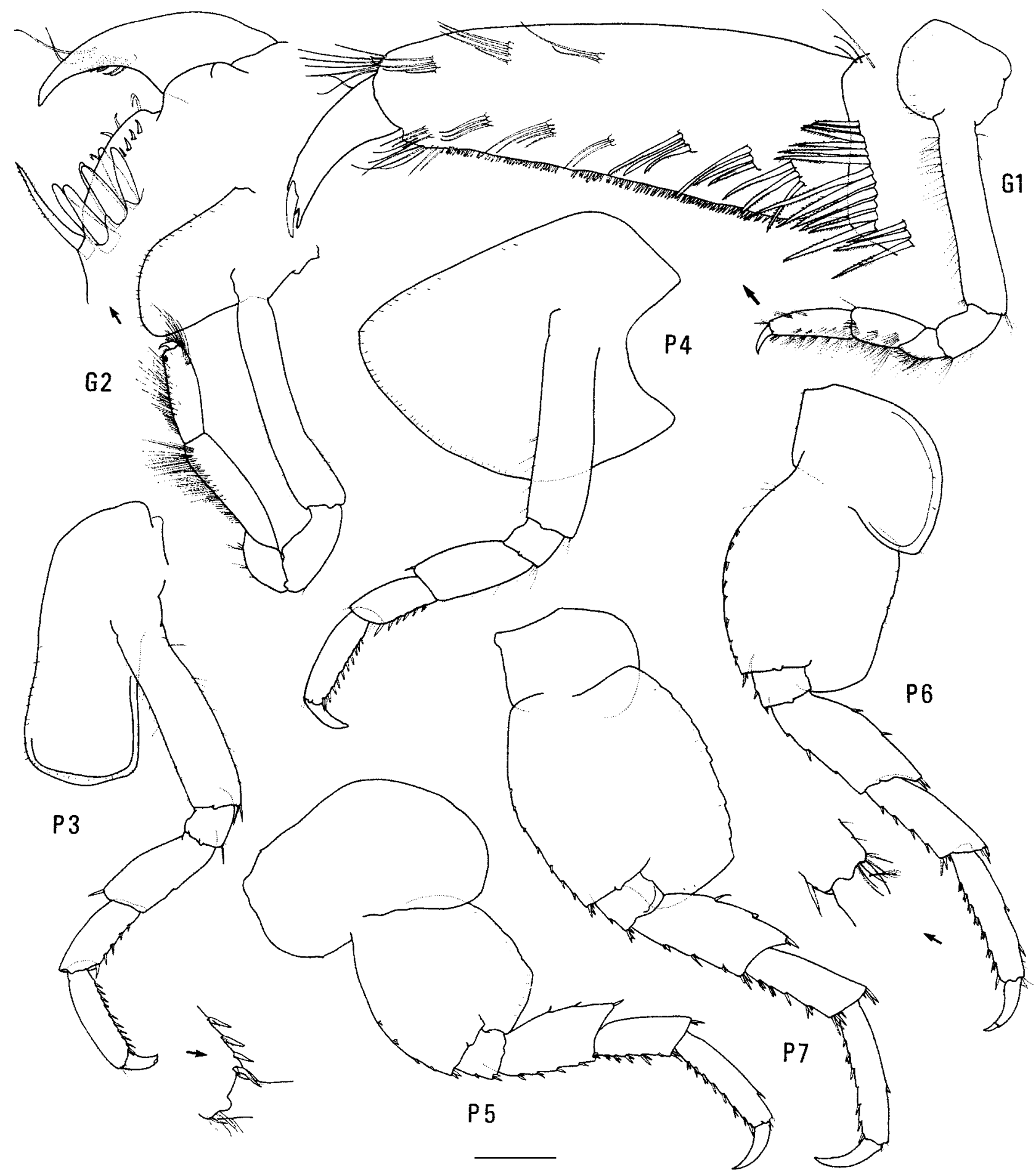

Figure 22. Amaryllis macrophthalma Haswell, neotype female, 21.4 mm, AM P37214, Derwent Estuary, Tasmania. Scales represent $0.5 \mathrm{~mm}$.

specimens, AM P37400, NMV J3325, NMV J3792 and J3795 to J3797, unknown localities in Western Port. 64 specimens from various localities in Port Phillip Bay, under the following registration numbers: NMV J3236 (1), J3237 (4), J3240 (2), J3242 (1), J3243 (4), J3246 (4), J3249 (1), J3250 (1), J3251 (4), J3252 (2), J3253 (1), J3255 (1), J3257 (1), J3258 (1), J3260 (6), J3264 (1), J3265 (1), J3266 (5), J3267 (9), J3268 (1), J3273 (4), J3332 (8), J3333 (1). BASS STRAIT: 48 specimens, AM E5674; 28 specimens, AM P2326; E of Flinders Island, eastern Bass Strait, [approx. 40 01 'S $\left.148^{\circ} 02^{\prime} \mathrm{E}\right]$, FIS Endeavour collection. 5 specimens, AM E6524 and 5 specimens, AM P5914, eastern slope, Bass Strait, FIS Endeavour collection. 1 specimen, NMV J7655, $42 \mathrm{~km} \mathrm{SSW} \mathrm{of} \mathrm{Lakes} \mathrm{Entrance,}$ Victoria, eastern Bass Strait, $38^{\circ} 18.5^{\prime} \mathrm{S} 147^{\circ} 37.5^{\prime} \mathrm{E}, 46 \mathrm{~m}$, muddy fine shell, otter trawl, M. Gomon \& R. Wilson, 31 July 1983, FV Silver Gull, stn BSS-210. 10 specimens, NMV J13995, 8 km S of South East Point, Wilsons Promontory, Victoria, central Bass Strait, $39^{\circ} 12.9^{\prime} \mathrm{S} 146^{\circ} 27.3^{\prime} \mathrm{E}$, $65 \mathrm{~m}$, medium sand, epibenthic sled, R. Wilson, 18 November 1981, RV Tangaroa, stn BSS-180. 1 specimen, NMV J13994, $43 \mathrm{~km} \mathrm{SE}$ of Port Albert, Victoria, eastern Bass Strait, $38^{\circ} 53.7^{\prime} \mathrm{S} 147^{\circ} 06.5^{\prime} \mathrm{E}, 58 \mathrm{~m}$, coarse shell, R. Wilson, 18 November 1981, RV Tangaroa, stn BSS-177. 1 specimen, NMV J13993, $33 \mathrm{~km}$ S of Deal Island, Tasmania, Central Bass Strait, $39^{\circ} 47.3^{\prime} \mathrm{S} 147^{\circ} 19.3^{\prime} \mathrm{E}, 60 \mathrm{~m}$, muddy sand, epibenthic sled, R. Wilson, 14 November 1981, RV Tangaroa, stn BSS-161. 4 specimens, NMV J13992, $57 \mathrm{~km} \mathrm{~S}$ of Rodondo Island, central Bass Strait, $39^{\circ} 46.0^{\prime} \mathrm{S}$ $146^{\circ} 18.0^{\prime} \mathrm{E}, 80 \mathrm{~m}$, muddy shell, epibenthic sled, R. Wilson, 13 November 1981, RV Tangaroa, cruise 81-T-1, stn BSS-159. 4 specimens, NMV $\mathrm{J} 7612,39 \mathrm{~km}$ NNE of Devenport, central Bass Strait, 40 $49.8^{\prime} \mathrm{S} 146^{\circ} 31.3^{\prime} \mathrm{E}$ 
to $40^{\circ} 48.2^{\prime} \mathrm{S} 146^{\circ} 33.7^{\prime} \mathrm{E}, 68-70 \mathrm{~m}$, mud, otter trawl, M.F. Gomon, G.C.B. Poore \& C.C. Lu, 4 February 1981, FRV Hai Kung, stn BSS-135. 11 specimens, NMV J13991, $5 \mathrm{~km}$ N of North Point, central Bass Strait, $40^{\circ} 40.3^{\prime} \mathrm{S} 145^{\circ} 15^{\prime} \mathrm{E}, 33 \mathrm{~m}$, medium shell, epibenthic sled, M. Gomon and G.C.B. Poore on FRV Sarda, 4 November 1980, stn BSS 115. 7 specimens, NMV J11263, $47 \mathrm{~km}$ E of Cape Rochon, Three Hummock Island, central Bass Strait, $40^{\circ} 23.8^{\prime} \mathrm{S} 145^{\circ} 32^{\prime} \mathrm{E}, 65 \mathrm{~m}$, muddy sand, epibenthic sled, M. Gomon \& G.C.B. Poore, 3 November 1980, stn BSS113. 17 specimens, NMV J13990, $23 \mathrm{~km}$ E of Cape Rochon, Three Hummock Island, central Bass Strait, $40^{\circ} 22.2^{\prime} \mathrm{S} 145^{\circ} 17^{\prime} \mathrm{E}, 40 \mathrm{~m}$, sand, epibenthic sled, M. Gomon \& G.C.B. Poore, 3 November 1980, FRV Sarda, stn BSS 112. 4 specimens, NMV J2312, $5 \mathrm{~km}$ E of Cape Edie, Robbins Island, central Bass Strait, $40^{\circ} 41.8^{\prime} \mathrm{S} 145^{\circ} 07^{\prime} \mathrm{E}, 16 \mathrm{~m}$, fine shelly sand, M. Gomon \& G.C.B. Poore, 3 November 1980, FRV Sarda, stn BSS-110. 63 specimens, NMV J13989, 9 km SSW of Cape Adansan, Three Hummock Island, central Bass Strait, 40 $30.9^{\prime} \mathrm{S} 144^{\circ} 56^{\prime} \mathrm{E}, 27 \mathrm{~m}$, very coarse sand, epibenthic sled, M. Gomon \& G.C.B. Poore, 2 November 1980, FRV Sarda, stn BSS 109. 23 specimens, NMV J2320 and 10 specimens, AM P37399, 28 km E of Cape Farewell, King Island, central Bass Strait, $39^{\circ} 32.8^{\prime} \mathrm{S} 144^{\circ} 16^{\prime} \mathrm{E}, 18 \mathrm{~m}$, fine sand, epibenthic sled, M. Gomon \& G.C.B. Poore, 1 November 1980, FRV Sarda, stn BSS-107. 2 specimens, NMV J11253, $59 \mathrm{~km} \mathrm{~W}$ of Stokes Point, King Island, western Bass Strait, $40^{\circ} 07^{\prime} \mathrm{S} 143^{\circ} 14^{\prime} \mathrm{E}, 185 \mathrm{~m}$, sandy mud, grab, G.C.B. Poore, 11 October 1980, HMAS Kimbla, stn BSS-104. TASMANIA: 1 male, AM E5680; 6 specimens, AM P5921; and 7 specimens, AM P18323; $16 \mathrm{~km} \mathrm{~N}$ of Circular Head, [approx. 40 40'S 145 $18^{\prime} \mathrm{E}$ ], 146-219 m, FIS Endeavour collection. 1 specimen, NMV J13997, $500 \mathrm{~m} \mathrm{~W}$ of Darlington, Maria Island, $42^{\circ} 35^{\prime} \mathrm{S} 148^{\circ} 02^{\prime} \mathrm{E}, 30 \mathrm{~m}$, algal and drift holdfast fauna, trawl, R.S. Wilson, 23 April 1985, RV Challenger, NMV stn TAS-27. 1 specimen, NMV J13998, same locality, 30 m, NMV stn TAS-33. 1 specimen, NMV J14000, E of Maria Island, 42 ${ }^{\circ} 36^{\prime} \mathrm{S} 148^{\circ} 10^{\prime} \mathrm{E}, 75 \mathrm{~m}$, fine bryozoans and shell, epibenthic sled, R.S. Wilson, 23 April 1985, RV Challenger, NMV stn TAS-30. 9 specimens, AM P37213, entrance to Oyster Bay, Maria Island, [approx. $42^{\circ} 41^{\prime} \mathrm{S} 148^{\circ} 02^{\prime} \mathrm{E}$ ], 29 July 1909 , K. Sheard Collection. 2 specimens, AM E6603, off Maria Island, 143 m, FIS Endeavour collection. 1 male, AM P37215 (illustrated specimen) and 5 females, AM P37216, type locality. 2 specimens, SAMA C5981,

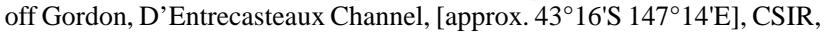
20 October 1938. 5 specimens, NMV J13999, $2.5 \mathrm{~km}$ SE of Birches Bay, D'Entrecasteaux Channel, $43^{\circ} 11^{\prime} \mathrm{S} 147^{\circ} 16^{\prime} \mathrm{E}, 10 \mathrm{~m}$, lip dredge, R.S. Wilson, 16 April 1985, RV Penghana, NMV stn TAS-1. 7 specimens, AM P37212, D'Entrecasteaux Channel, 9 m, dredged on scallop banks, K. Sheard Collection. South Australia: 6 specimens, AM P59046, Nepean Bay, Kangaroo Island, [approx. $35^{\circ} 42^{\prime} \mathrm{S} 137^{\circ} 37^{\prime} \mathrm{E}$ ], dredged, F. Moorhouse, May 1938. 1 specimen, SAMA C5982, 8 miles W of Halletts Cove, Gulf St Vincent, [approx. $35^{\circ} 05^{\prime} \mathrm{S} 138^{\circ} 30^{\prime} \mathrm{E}$ ], tow net, H.M. Hale \& K. Sheard, 14 April 1945. 3 specimens, SAMA C5983, Dangerous Reef, Spencer Gulf, [approx. $34^{\circ} 49^{\prime} \mathrm{S} 136^{\circ} 12^{\prime} \mathrm{E}$ ], dredged, K. Sheard. 11 specimens, AM P59047, unknown locality in Spencer Gulf and/or Gulf St Vincent.

Type locality. Derwent Estuary, D'Entrecasteaux Channel, Tasmania, Australia, [approx.42 ${ }^{\circ} 54^{\prime} \mathrm{S} 147^{\circ} 23^{\prime} \mathrm{E}$ ].

Description. Based on neotype, female, $21.5 \mathrm{~mm}$, ovigerous (14 eggs), AM P37214. Head much deeper than long, anterior margin with notch extended into a slit; rostrum absent; eye present, elongate, reniform. Antenna 1 peduncular article 1 not ball-shaped proximally, distal margin with well-developed medial spine; peduncular article 2 medium length; flagellum without callynophore, calceoli absent. Antenna 2 flagellum about as long as that of antenna 1 , without calceoli. Mouthpart bundle subconical. Epistome/ upper lip with broad mid-anterior bulge (lateral view). Mandible lacinia mobilis a stemmed, distally-cusped blade; accessory setal row with intermediate setae; palp article 2 with 5 posterodistal setae, article 3 without A3-seta. Maxilliped outer plate with distal margin smooth, medial margin without notch.

Gnathopod 1 carpus subequal in length to propodus $(0.9 \times)$; propodus, posterior margin without robust setae. Gnathopod 2 palm slightly acute, with 2-4 lateral robust setae, 2-3 medial robust setae. Pereopods 3 and 4 merus and carpus without setal fringe. Pereopod 4 coxa with anterior and posterior margins subparallel, anteroventral corner rounded. Pereopods 5-7 with distal articles elongate, dactyls short and stocky. Pereopod 5 basis expanded posteriorly, rounded. Pereopod 7 basis subrectangular, posteroventral corner subquadrate, posteroventral margin straight.

Epimeron 3 posterior margin smooth, with notch well above rounded posteroventral corner. Uropod 1 peduncle dorsolateral margin with 18 robust setae; outer ramus without large spines between robust setae. Uropod 2 inner ramus slightly constricted. Uropod 3 rami lanceolate; without plumose setae; outer ramus 1-articulate. Telson moderately cleft (about 50\%).

Male (sexually dimorphic characters). Based on male, 17.8 $\mathrm{mm}, \mathrm{AM}$ P37215. Antenna 1 flagellum with callynophore (reduced). Antenna 2 flagellum with calceoli. Mandible palp article 2 with 14 posterodistal setae. Gnathopod 2 palm with 1 lateral robust seta and 2 medial robust setae.

Remarks. Amaryllis macrophthalma belongs to a small group of species with a smooth posterior margin on epimeron 3 and a subrectangular basis on pereopod 7 ( $A$. macrophthalma, A. philatelica and A. spencerensis). Within this group A. macrophthalma and A. spencerensis both have a well-developed medial spine on peduncular article 1 of antenna 1 and a notch well above the rounded posteroventral corner on epimeron 3. In Amaryllis macrophthalma the posteroventral corner of the basis of pereopod 7 is subquadrate but not notched (it is notched in A. spencerensis), and there are more robust setae defining the palm of gnathopod 2 in the female.

Habitat. Amaryllis macrophthalma occurs most frequently on sand, shell and mud bottoms of both fine and coarse sediment.

Distribution. Southeastern Australia, predominantly Bass Strait and Tasmania; 6-219 m depth.

\section{Amaryllis migo n.sp.}

Figs. 23-25

Type material. HolOTYPE, female, $10.2 \mathrm{~mm}$, ovigerous (with 1 embryo), AM P37173; 1 PARATYPE, male, $5.2 \mathrm{~mm}$, AM P37174; 3 PARATYPES, AM P37175; rocks near Migo Island, Port Harding, Torbay Bay, Western Australia, $35^{\circ} 04^{\prime} \mathrm{S} 117^{\circ} 39^{\prime} \mathrm{E}, 6-7 \mathrm{~m}$, algae and sponge, J.K. Lowry, 15 December 1983, stn WA-154. 11 PARATYPES, AM P37176, type locality, 6-7 m, small branched alga with compound ascidian on underside of branches, R.T. Springthorpe \& J.K. Lowry, 15 December 1983, stn WA-152.

Additional material. South Australia: 1 specimen, AM P37177, Vivonne Bay, Kangaroo Island, [approx. $36^{\circ} 00^{\prime} S 137^{\circ} 05^{\prime} E$ ], H.M. Hale and Tindale, January 1926, AM stn SA-85. 7 specimens, NMV J13978, $\mathrm{NE}$ side of Topgallant Island, Investigator Group, $33^{\circ} 43.0^{\prime} \mathrm{S} 134^{\circ} 36.6^{\prime} \mathrm{E}$, $20 \mathrm{~m}$, a brown alga Cystophora and a red alga, Plocamium, G.C.B. Poore \& K. Brandon, 21 April 1985, NMV stn SA-80. 1 specimen, NMV J13979, same locality, $7 \mathrm{~m}$, Acrocarpia anciculata and red algae, S. Shepherd \& G.C.B. Poore, 20 April 1985, NMV stn SA-83. 2 specimens, NMV J13985, The Hotspot Reef, W of Flinders Island, $33^{\circ} 40.8^{\prime} \mathrm{S} 134^{\circ} 22.5^{\prime} \mathrm{E}$, $21 \mathrm{~m}$, erect hard bryozoan on vertical rock face, G.C.B. Poore, 22 April 1985, NMV stn SA-83. WESTERN AUSTRALIA: 3 specimens, NMV J13986, $\mathrm{N}$ side of Cape Riche, $34^{\circ} 37^{\prime} \mathrm{S} 118^{\circ} 47^{\prime} \mathrm{E}, 5 \mathrm{~m}$, coralline and red algae, G.C.B. Poore \& H.M. Lew Ton, 14 April 1985, NMV stn SWA-51. 27 


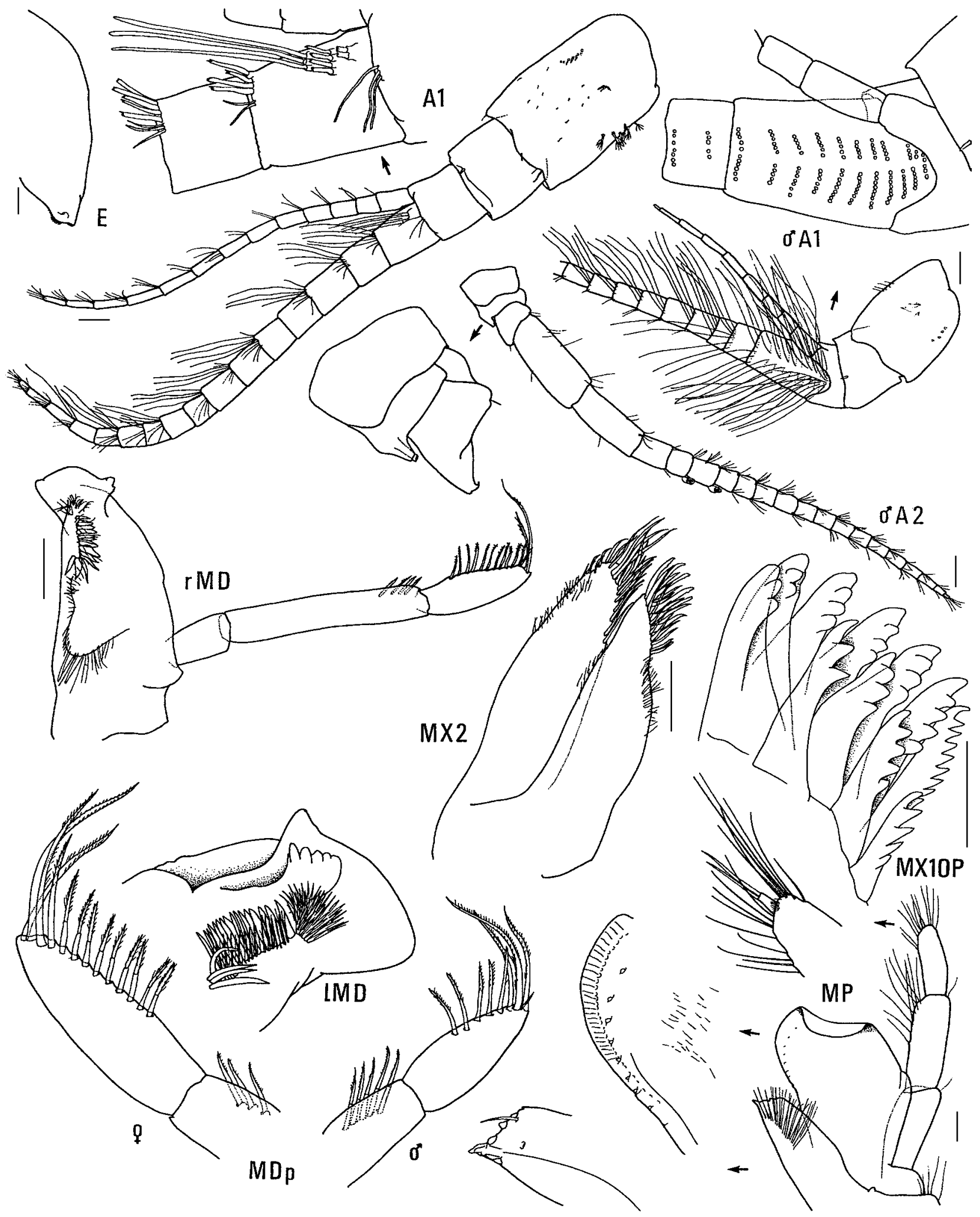

Figure 23. Amaryllis migo n.sp., holotype female, $10.2 \mathrm{~mm}$, AM P37173; paratype male, $5.2 \mathrm{~mm}$, AM P37174; Torbay Bay, Western Australia. Scale for MX1OP represents $0.05 \mathrm{~mm}$; remainder represent $0.1 \mathrm{~mm}$.

specimens, AM P37178, $2 \mathrm{~km} \mathrm{SE}$ of South Point, Two Peoples Bay, $34^{\circ} 58^{\prime} \mathrm{S} 118^{\circ} 12^{\prime} \mathrm{E}, 6-10 \mathrm{~m}$, alga with compound ascidian on back of fronds, J.K. Lowry, 16 December 1983, stn WA-177.

Type locality. Migo Island, Port Harding, Torbay Bay, Western Australia, $35^{\circ} 04^{\prime} \mathrm{S} 117^{\circ} 39^{\prime} \mathrm{E}, 6-7 \mathrm{~m}$ depth.
Description. Based on holotype female, $10.2 \mathrm{~mm}$. Head much deeper than long, anterior margin with notch extended into a slit; rostrum absent; eye present, elongate, reniform. Antenna 1 peduncular article 1 not ball-shaped proximally, distal margin without a medial spine; peduncular article 2 medium length; flagellum with weak callynophore, calceoli 


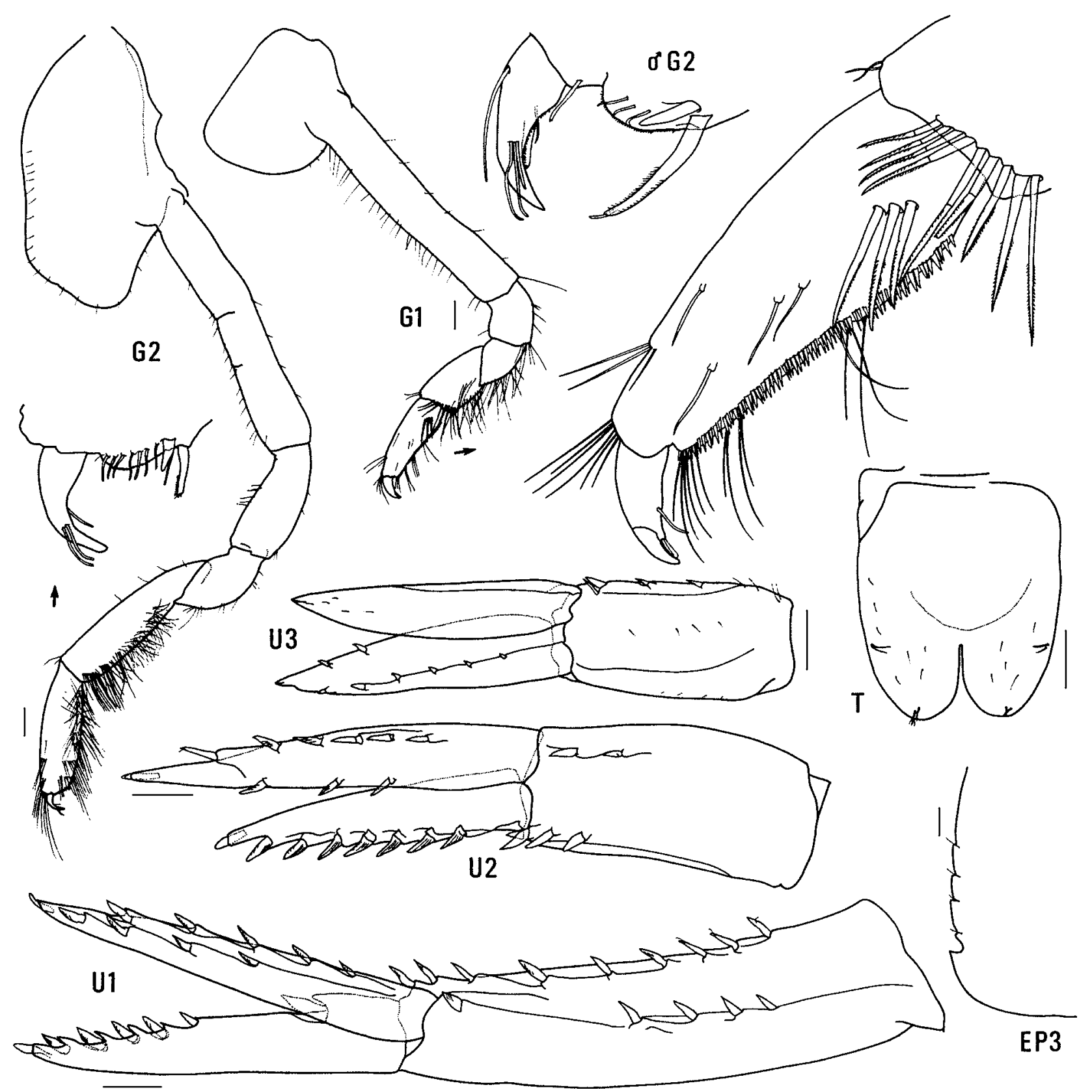

Figure 24. Amaryllis migo n.sp., holotype female, $10.2 \mathrm{~mm}$, AM P37173; paratype male, $5.2 \mathrm{~mm}$, AM P37174; Torbay Bay, Western Australia. Scales represent $0.1 \mathrm{~mm}$.

absent. Antenna 2 flagellum about as long as that of antenna 1, without calceoli. Mouthpart bundle subconical. Epistome/ upper lip with broad mid-anterior bulge (lateral view). Mandible lacinia mobilis a stemmed, distally-cusped blade; accessory setal row with intermediate setae; palp article 2 with 3 posterodistal setae, article 3 without A3-seta. Maxilliped outer plate with distal margin smooth, medial margin without notch.

Gnathopod 1 carpus subequal in length to propodus; propodus, posterior margin without robust setae. Gnathopod 2 palm slightly acute, with no or 1 lateral robust seta, 1 medial robust seta. Pereopods 3 and 4 merus and carpus without setal fringe. Pereopod 4 coxa with anterior and posterior margins subparallel, anteroventral corner rounded. Pereopods 5-7 with distal articles elongate, dactyls short and stocky. Pereopod 5 basis expanded posteriorly, rounded. Pereopod 7 basis rounded posteriorly, posteroventral corner rounded, posteroventral margin curved.

Epimeron 3 posterior margin serrate, with notch well above rounded posteroventral corner. Uropod 1 peduncle dorsolateral margin with 9 robust setae; outer ramus with large spines between robust setae. Uropod 2 inner ramus slightly constricted. Uropod 3 rami lanceolate; without plumose setae; outer ramus 1 -articulate. Telson moderately cleft (about 32\%).

Male (sexually dimorphic characters). Based on paratype, male, $5.2 \mathrm{~mm}$, AM P37174. Antenna 1 with strong callynophore. Antenna 2 flagellum with calceoli. Mandible palp article 2 with 5 posterodistal setae. Gnathopod 2 palm without lateral robust setae.

Etymology. The species is named after its type locality, Migo Bay. 


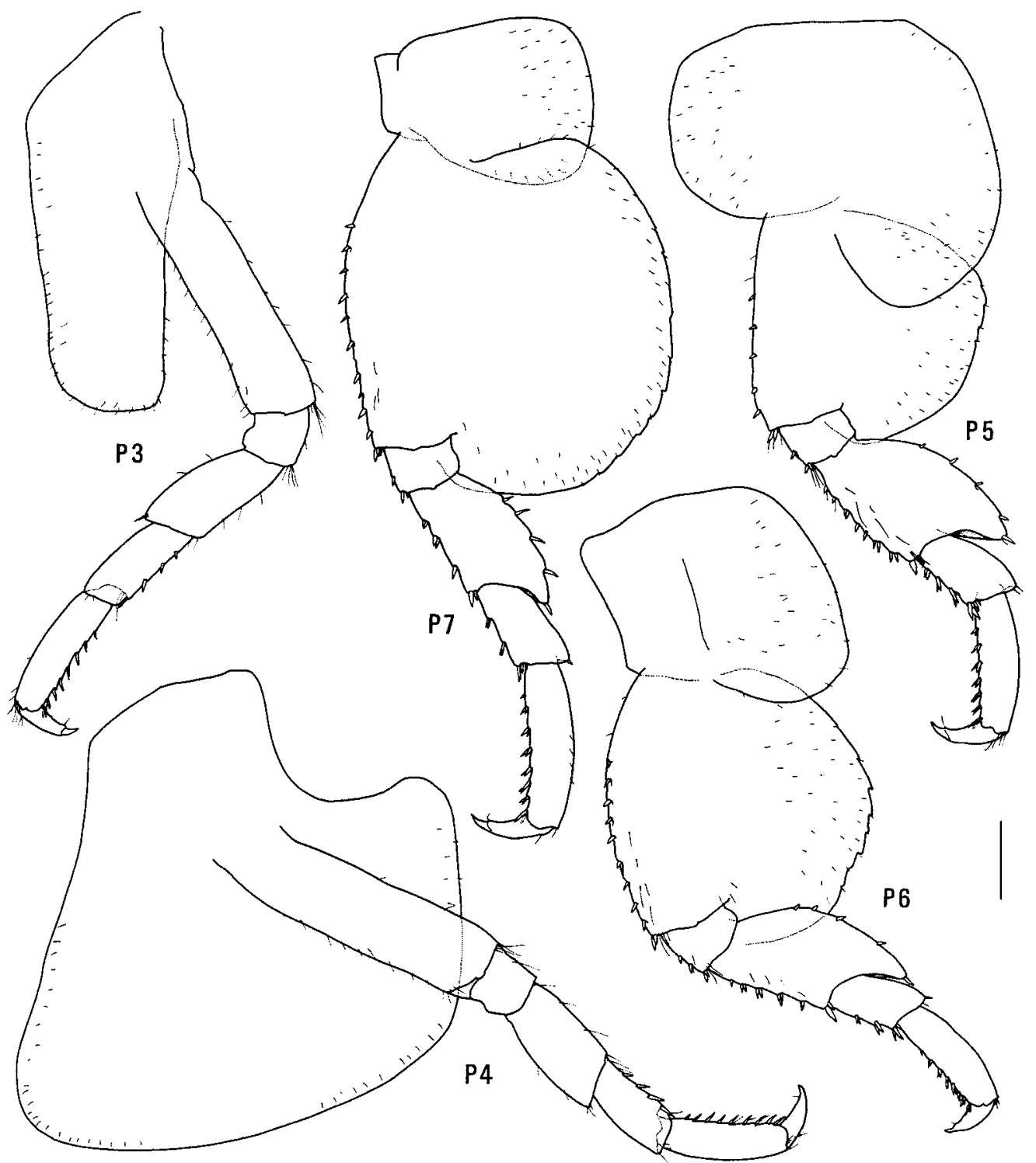

Figure 25. Amaryllis migo n.sp., holotype female, 10.2 mm, AM P37173, Torbay Bay, Western Australia. Scales represent $0.5 \mathrm{~mm}$.

Remarks. Amaryllis migo is most similar to other Amaryllis species with a serrate posterior margin on epimeron 3 , such as A. carrascoi, A. croca, A. dianae and A. keablei. Amaryllis dianae, A. carrascoi and A. migo all have a notch well above the posteroventral corner of epimeron 3, and only small serrations above the notch. Amaryllis migo is the only one of these three species with a rounded posteroventral corner on the basis of pereopod 7; it is the only species of Amaryllis with large spines between the robust setae of uropod 1 outer ramus.

Habitat. Amaryllis migo has been collected mostly from algae.

Distribution. Southern coast of Australia; 5-21 m depth.

\section{Amaryllis moona n.sp.}

Figs. 26, 27

Type material. HOLOTYPE, female, 8.2 mm, AM P37243; 2 PARATYPES, male, AM P37371; Summercloud Bay, Wreck Bay, New South Wales, Australia, $35^{\circ} 10^{\prime} \mathrm{S} 150^{\circ} 42^{\prime} \mathrm{E}, 9-15$ $\mathrm{m}$, sponges, ascidians and algal holdfasts on underside of rocks, P. Hutchings, 29 November 1971, stn NSW-228. 1 PARATYPE, male, $4.8 \mathrm{~mm}$, AM P37372, off Moona Moona Creek, Jervis Bay, NSW, Australia, Tasman Sea, $35^{\circ} 03.5^{\prime} \mathrm{S}$ $150^{\circ} 41^{\prime} \mathrm{E}, 8 \mathrm{~m}$, algae and sponge on sandy rocks and mussels, J.K. Lowry, 19 June 1982, stn NSW-113.

Type locality. Summercloud Bay, Wreck Bay, NSW, Australia, $35^{\circ} 10^{\prime} \mathrm{S} 150^{\circ} 42^{\prime} \mathrm{E}, 9-15 \mathrm{~m}$ depth.

Description. Based on holotype female, $8.2 \mathrm{~mm}$, AM P37243. Head much deeper than long, anterior margin with notch extended into a slit; rostrum absent; eye present, elongate, reniform. Antenna 1 peduncular article 1 not ballshaped proximally, distal margin with small medial spine; peduncular article 2 medium length; flagellum without 


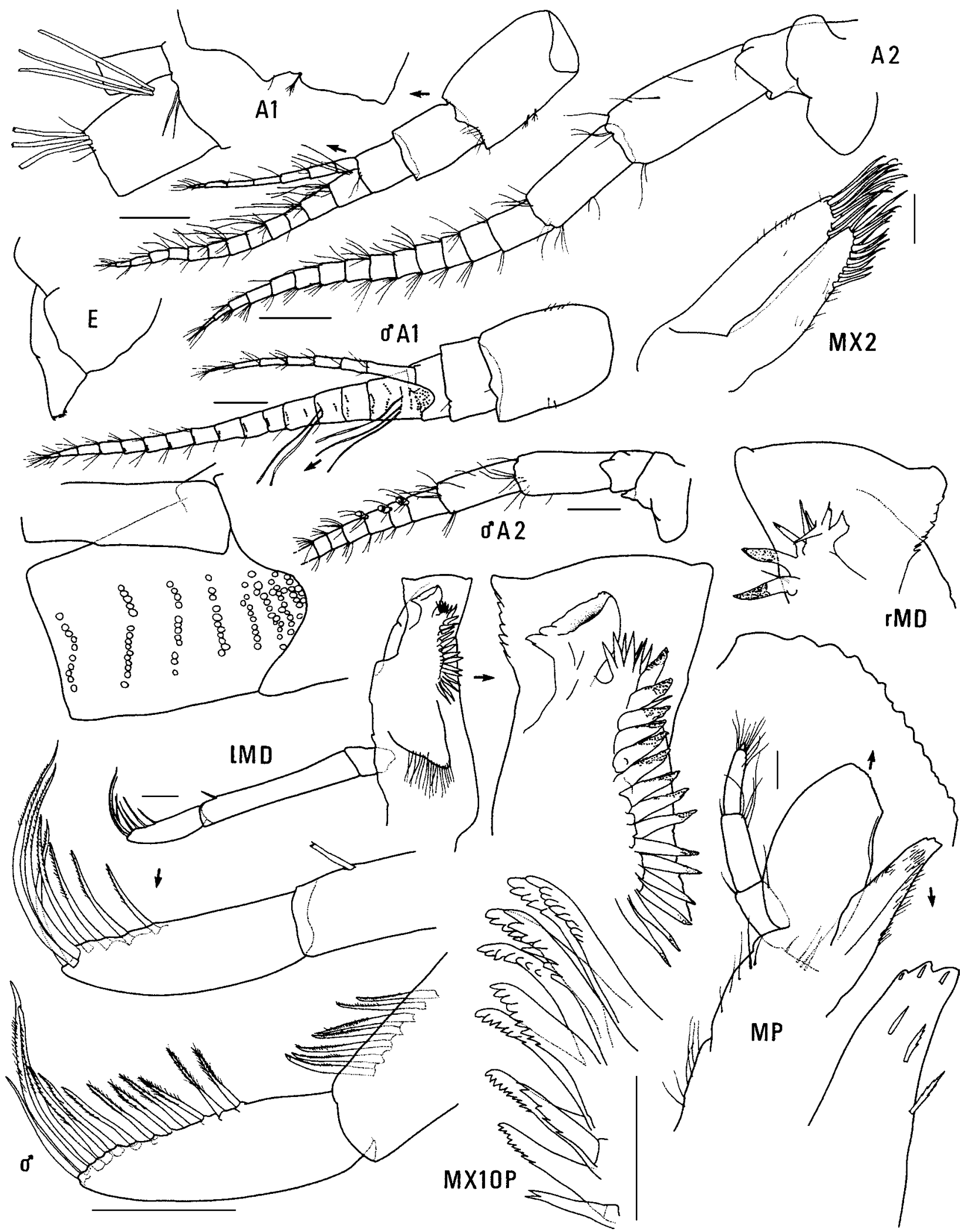

Figure 26. Amaryllis moona n.sp., holotype female, $8.2 \mathrm{~mm}$, AM P37243; paratype male, 6.4 mm, AM P37371; Summercloud Bay, NSW. Scales for A1, 2 represent $0.2 \mathrm{~mm}$; remainder represent $0.1 \mathrm{~mm}$.

callynophore, calceoli absent. Antenna 2 flagellum about as long as that of antenna 1, without calceoli. Mouthpart bundle subconical. Epistome/upper lip almost straight (lateral view). Mandible lacinia mobilis a stemmed cuplike blade; accessory setal row without intermediate setae; palp article 2 with 1 posterodistal seta, article 3 without
A3-seta. Maxilliped outer plate with distal margin serrate, medial margin without notch.

Gnathopod 1 carpus longer than propodus $(1.2 \times)$; propodus, posterior margin without robust setae. Gnathopod 2 palm slightly acute, without lateral robust setae, 1 medial robust seta. Pereopods 3 and 4 merus and carpus without 


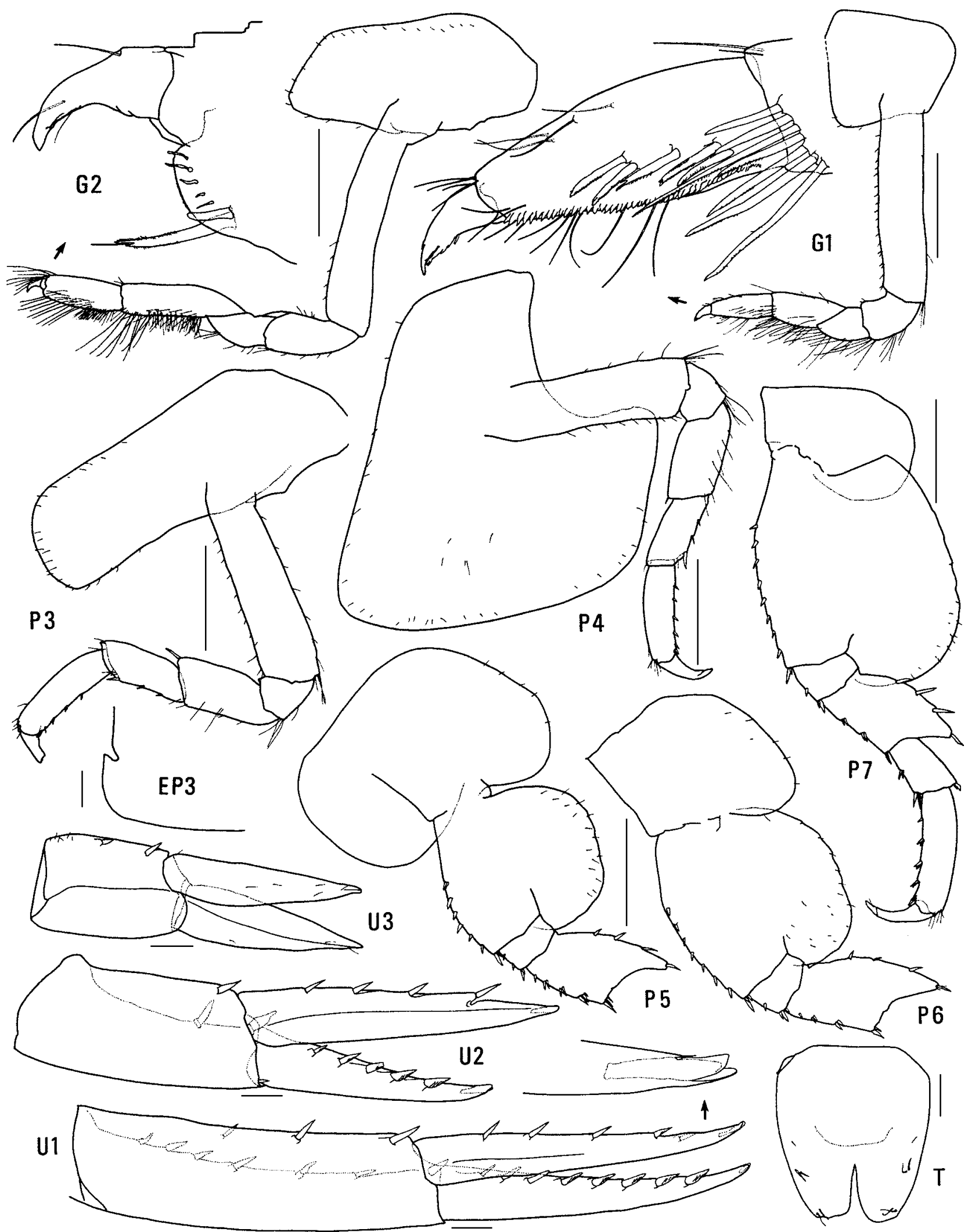

Figure 27. Amaryllis moona n.sp., holotype female, 8.2 mm, AM P37243; Summercloud Bay, NSW. Scales for G1, 2, P3-7 represent $0.5 \mathrm{~mm}$; remainder represent $0.1 \mathrm{~mm}$.

setal fringe. Pereopod 4 coxa with anterior and posterior margins subparallel, anteroventral corner rounded. Pereopods 5-7 with distal articles elongate, dactyls short and stocky. Pereopod 5 basis expanded posteriorly, rounded.
Pereopod 7 basis rounded posteriorly, posteroventral corner rounded, posteroventral margin curved.

Epimeron 3 posterior margin smooth, with notch well above rounded posteroventral corner. Uropod 1 peduncle 
dorsolateral margin with 7 robust setae; outer ramus without large spines between robust setae. Uropod 2 inner ramus slightly constricted. Uropod 3 rami lanceolate; without plumose setae; outer ramus 1-articulate. Telson moderately cleft (about 34\%).

Male (sexually dimorphic characters). Based on paratype male, $4.8 \mathrm{~mm}$, AM P37372. Antenna 1 flagellum with callynophore. Antenna 2 flagellum with calceoli. Mandible palp article 2 with 7 posterodistal setae.

Etymology. Named for Moona Moona Creek.

Remarks. Amaryllis moona belongs to the group of species with a smooth posterior margin on epimeron 3 and a rounded posteroventral corner on the basis of pereopod 7. Within this group it is similar to A. brevicornis in having a nearly straight upper lip, but $A$. moona has significantly less robust setae at the corner of the palm of gnathopod 2 than does $A$. brevicornis. Amaryllis moona and A. quokka are the only two Amaryllis species with a cup-shaped lacinia mobilis; A. moona can be distinguished from A. quokka by the serrate distomedial margin of the maxilliped outer plate and the slightly acute palm on gnathopod 2 .

Habitat. Amaryllis moona has been found only rarely, among mixed algae and sedentary invertebrates.

Distribution. Known only from the Jervis Bay area on the southeast coast of Australia; 8-15 m depth.

\section{Amaryllis olinda n.sp.}

Figs. 28-30

Type material. HOLOTYPE, female, $8.9 \mathrm{~mm}$, ovigerous, AM P37169; 1 PARATYPE, male, 5.5 mm, AM P37170; 10 PARATYPES, AM P37171; Port Arthur, Tasmania, Australia, [approx. 4309'S $147^{\circ} 51^{\prime} \mathrm{E}$ ], collected 28 July 1909, ex Keith Sheard Collection.
Additional material. BASS STRAIT: 1 specimen, AM E6523, east coast of Flinders Island, eastern Bass Strait, [approx. $40^{\circ} 01^{\prime} \mathrm{S} 148^{\circ} 02^{\prime} \mathrm{E}$ ], FIS Endeavour collection. TASMANIA: 3 specimens, AM P37172, entrance to Oyster Bay, Maria Island, [approx. $42^{\circ} 40^{\prime} \mathrm{S} 148^{\circ} 03^{\prime} \mathrm{E}$ ], collected 29 July 1909. 148 specimens, NMV J7606, $60 \mathrm{~km}$ ENE of Bold Head, King Island, Central Bass Strait, Australia, $39^{\circ} 56.4^{\prime} \mathrm{S} 144^{\circ} 48.1^{\prime} \mathrm{E}, 49 \mathrm{~m}$, coarse shell, M.F. Gomon, G.C.B. Poore \& C.C. Lu, 3 February 1981, FRV Hai Kung, stn BSS-128. 4 specimens, NMV J13988, 5 km E of Cape Edie, Robbins Island, Bass Strait, $40^{\circ} 41.8^{\prime} \mathrm{S} 145^{\circ} 07^{\prime} \mathrm{E}, 16 \mathrm{~m}$, fine shelly sand, epibenthic sled, M. Gomon \& G.C.B. Poore, 3 November 1980, FRV Sarda, stn BSS-110S.

Type locality. Port Arthur, Tasmania, Australia, Southern Ocean, [approx. $43^{\circ} 09^{\prime} \mathrm{S} 147^{\circ} 51^{\prime} \mathrm{E}$ ].

Description. Based on holotype female, $8.9 \mathrm{~mm}$, AM P37169. Head much deeper than long, anterior margin with notch extended into a slit; rostrum absent; eye present, elongate, reniform. Antenna 1 peduncular article 1 not ballshaped proximally, distal margin without a medial spine; peduncular article 2 medium length; flagellum with callynophore, calceoli absent. Antenna 2 flagellum about as long as that of antenna 1, without calceoli. Mouthpart bundle subconical. Epistome/upper lip with broad midanterior bulge (lateral view). Mandible lacinia mobilis a stemmed, distally-cusped blade; accessory setal row with intermediate setae; palp article 2 with 7 posterodistal setae, article 3 without A3-seta. Maxilliped outer plate with distal margin smooth, medial margin without notch.

Gnathopod 1 carpus shorter than propodus $(0.75 \times)$; propodus, posterior margin without robust setae. Gnathopod 2 palm slightly acute, with 1 lateral robust seta, 1 medial robust seta. Pereopods 3 and 4 merus and carpus without setal fringe. Pereopod 4 coxa with anterior and posterior margins subparallel, anteroventral corner rounded. Pereopods 5-7 with distal articles elongate, dactyls short and stocky. Pereopod 5 basis expanded posteriorly, rounded. Pereopod 7 basis rounded posteriorly, posteroventral corner rounded, posteroventral margin curved.

Epimeron 3 posterior margin smooth, with notch well above rounded posteroventral corner. Uropod 1 peduncle

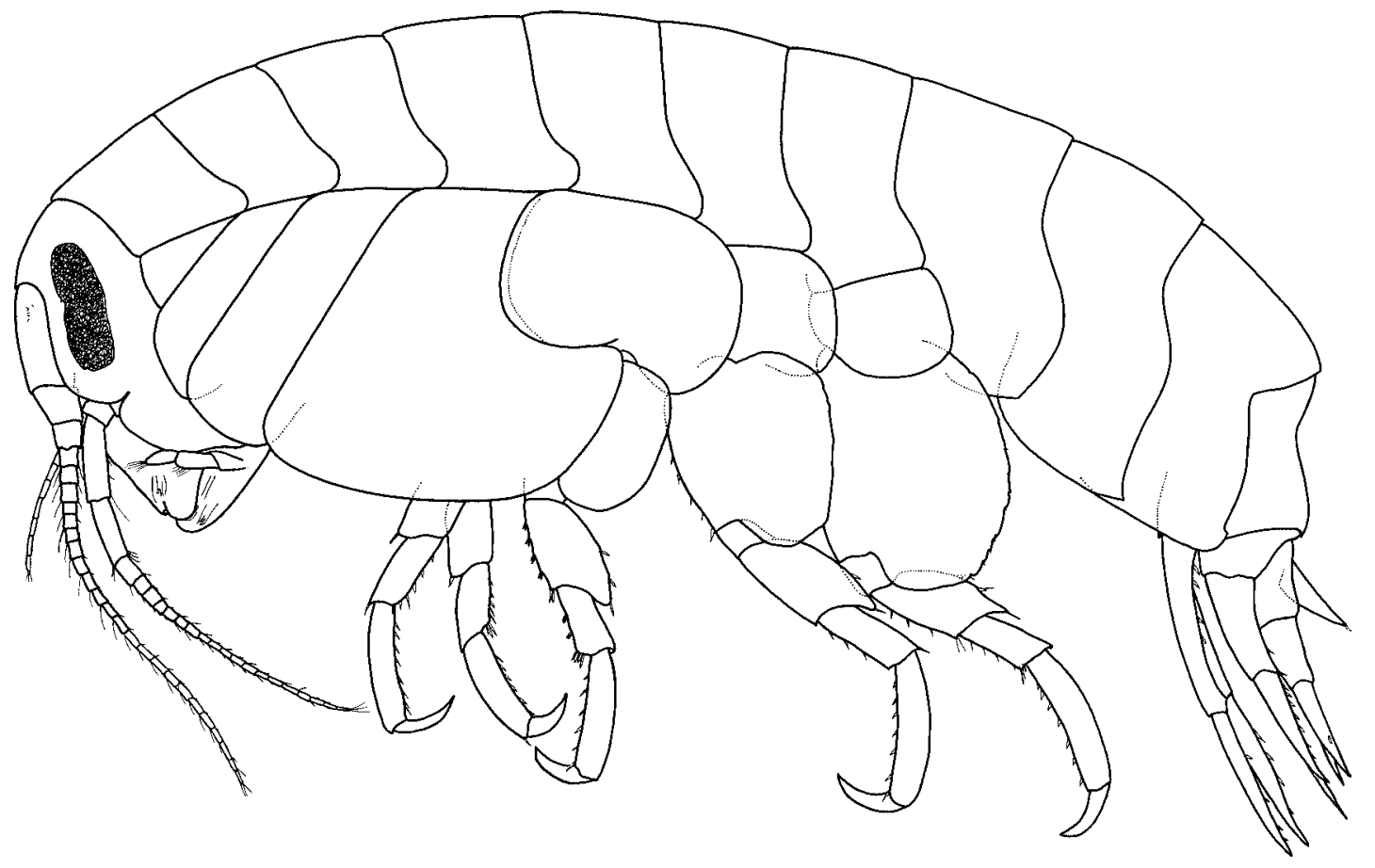

Figure 28. Amaryllis olinda n.sp., holotype female, 8.9 mm, AM P37169, Port Arthur, Tasmania. 


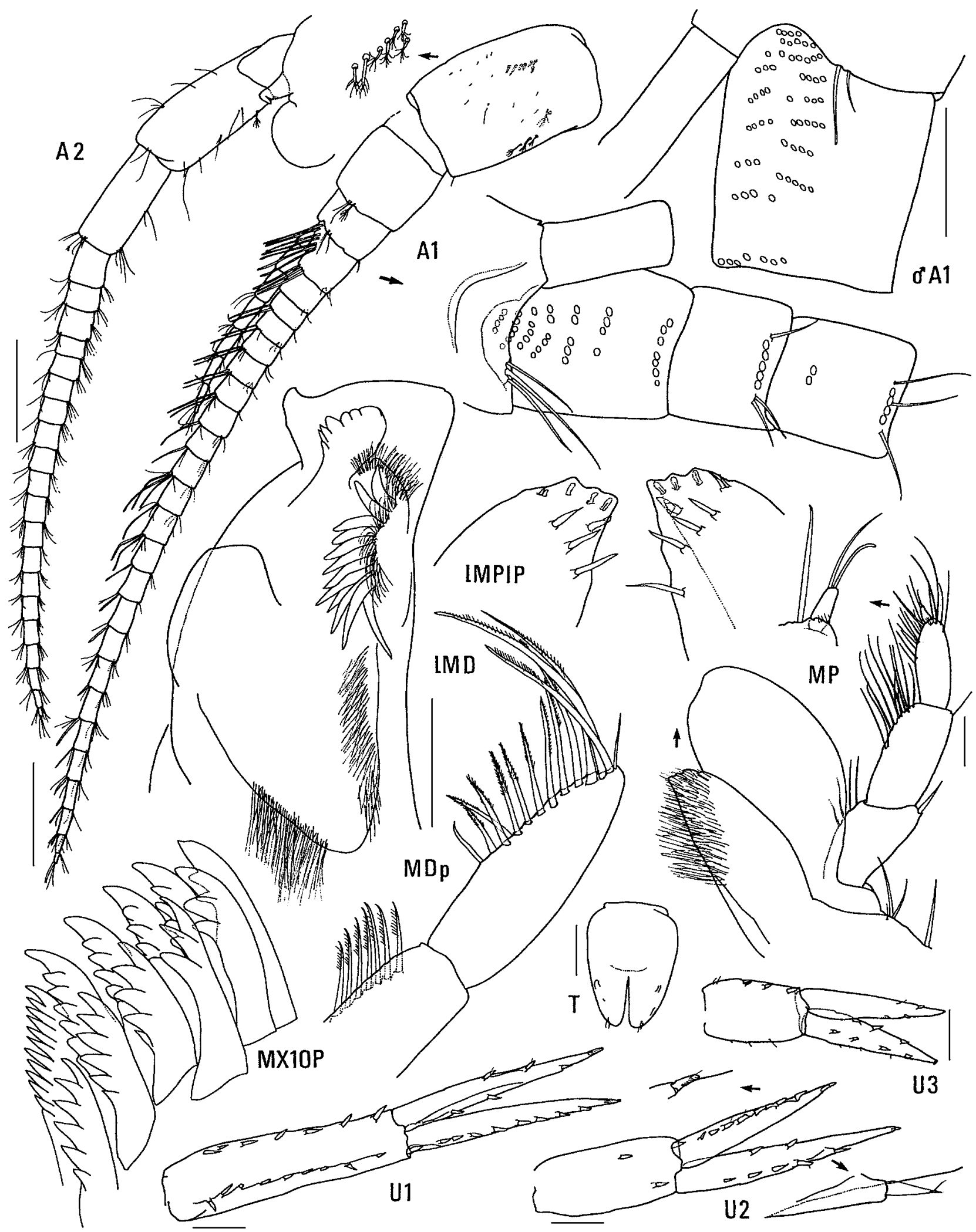

Figure 29. Amaryllis olinda n.sp., holotype female, $8.9 \mathrm{~mm}$, AM P37169; paratype male, $5.5 \mathrm{~mm}$, AM P37170; Port Arthur, Tasmania. Scales for MD, MP, ô A1 represent $0.1 \mathrm{~mm}$; remainder represent $0.2 \mathrm{~mm}$.

dorsolateral margin with 9 robust setae; outer ramus without large spines between robust setae. Uropod 2 inner ramus slightly constricted. Uropod 3 rami lanceolate; without plumose setae; outer ramus 1-articulate. Telson moderately cleft (about 40\%).
Male (sexually dimorphic characters). Based on paratype male, $5.5 \mathrm{~mm}$, AM P37170. Antenna 1 flagellum with calceoli. Antenna 2 flagellum with calceoli. Mandible palp article 2 with 5 posterodistal setae. Gnathopod 2 palm without lateral robust setae. 


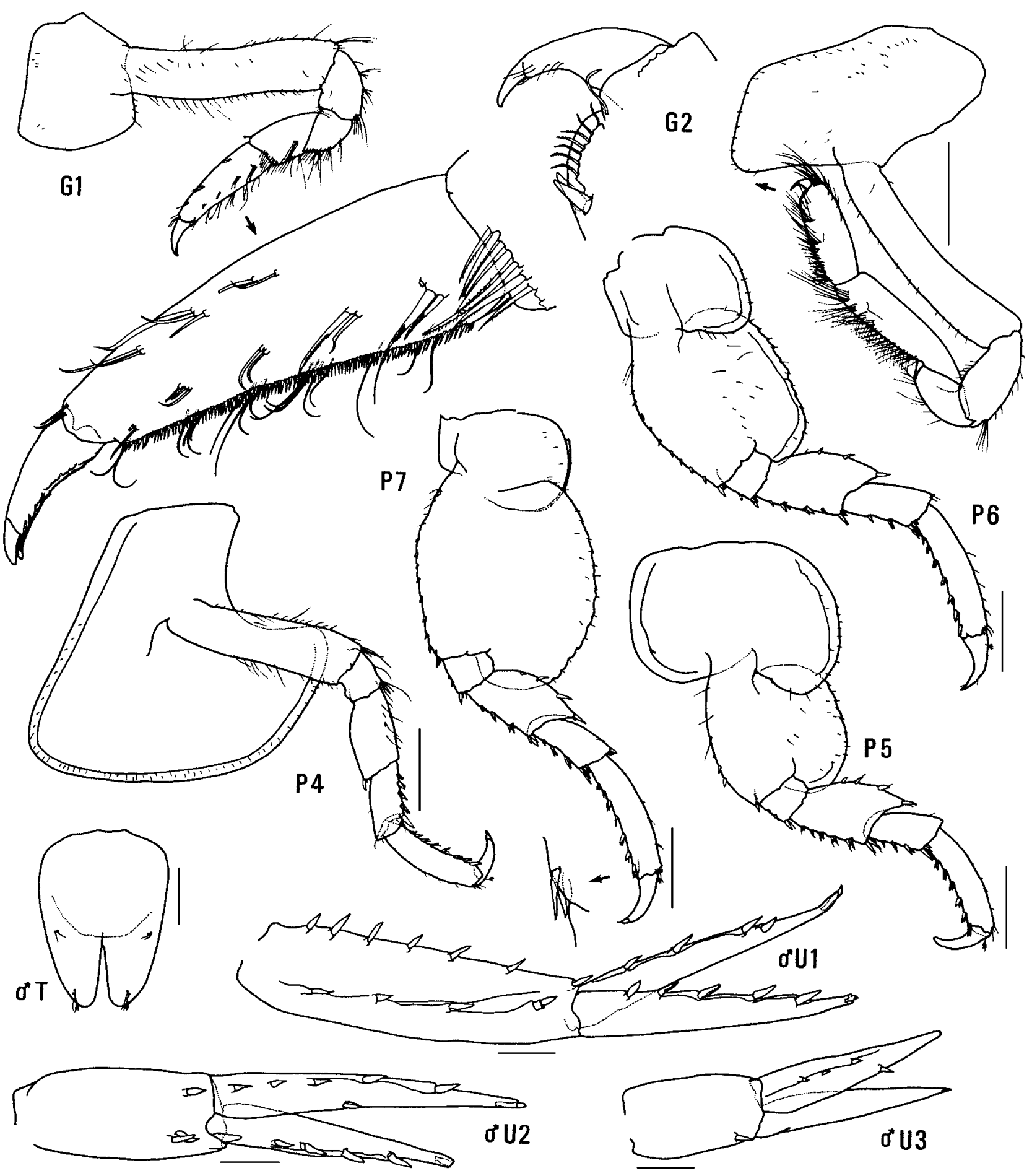

Figure 30. Amaryllis olinda n.sp., holotype female, 8.9 mm, AM P37169; paratype male, 5.5 mm, AM P37170; Port Arthur, Tasmania. Scales for U1-3, T represent $0.1 \mathrm{~mm}$; remainder represent $0.5 \mathrm{~mm}$.

Etymology. The species is named for the wooden barque Olinda, which sank in 1852 off Eddystone Point, Tasmania, while carrying a cargo of timber and potatoes from Hobart to Melbourne.

Remarks. Amaryllis olinda belongs to the group of species with a smooth posterior margin on epimeron 3 and a rounded posteroventral corner on the basis of pereopod 7 ( $A$. brevicornis, A. kamata, A. moona, A. quokka). It has a curved posteroventral margin on the basis of pereopod 7, similar to A. moona and A. quokka. It differs from these two species in having gnathopod 1 carpus shorter than the propodus.

Habitat. Amaryllis olinda has been collected from coarse sediments.

Distribution. Tasmania, Australia; $16-49$ m depth. 


\section{Amaryllis philatelica n.sp.}

Figs. 31-34, Plate 1b

Type material. HOLOTYPE, female, 20.5 mm, AM P37165; 1 PARATYPE, male, $18.2 \mathrm{~mm}$, AM P37166; 2 PARATYPES, males, AM P37167; Montague Island, New South Wales, Australia, $36^{\circ} 15^{\prime} \mathrm{S} 150^{\circ} 14^{\prime} \mathrm{E}, 23 \mathrm{~m}$, R. Kuiter, 28 December 1978. 1 PARATYPE, female, AM P26644, Montague Island, NSW, Australia, $36^{\circ} 15^{\prime} \mathrm{S} 150^{\circ} 14^{\prime} \mathrm{E}, 25 \mathrm{~m}$, algae, R. Kuiter, 30 December 1977.

Additional material. New South Wales: 1 male, AM P30479, Point Perpendicular, Jervis Bay, $35^{\circ} 06^{\prime} \mathrm{S} 150^{\circ} 48^{\prime} \mathrm{E}, 20 \mathrm{~m}$, on boulder surface, A. Jones \& R. Hartnall, 25 May 1980. BASS STRAIT: 1 specimen, NMV $\mathrm{J} 7653,40 \mathrm{~km} \mathrm{SSW}$ of Lakes Entrance, $38^{\circ} 18.0^{\prime} \mathrm{S} 147^{\circ} 37.0^{\prime} \mathrm{E}, 55 \mathrm{~m}$, muddy fine shell, epibenthic sled, M. Gomon \& R. Wilson, 31 July 1983, FV Silver Gull, stn BSS-209. TASMANIA: 5 specimens, AM P57882, Waterfall Bay, Tasman Peninsula, $43^{\circ} 04^{\prime} \mathrm{S} 147^{\circ} 56^{\prime} \mathrm{E}, 17-18 \mathrm{~m}$, bryozoans and sponges on rock wall, K.L. Gowlett-Holmes, 7 December 1997. SouTH AUSTRALIA: 3 specimens, SAMA C5984, $2 \mathrm{~km} \mathrm{~S} \mathrm{of} \mathrm{SW} \mathrm{tip} \mathrm{of} \mathrm{West} \mathrm{Island,}$ Franklin Island, Nuyts Archipelago, $32^{\circ} 27^{\prime} \mathrm{S} 133^{\circ} 40^{\prime} \mathrm{E}, 36.5 \mathrm{~m}$, sand with low rock outcrops, P. Aerfeldt \& N. Holmes, 23 February 1983. WESTERN Australia: 1 specimen, AM P37168, SE of Foul Bay, $35^{\circ} 12^{\prime} \mathrm{S} 117^{\circ} 00^{\prime} \mathrm{E}$, 75 m, CSIRO Fisheries, 8 August 1962, HMAS Gascoyne, stn G3/160/62.

Type locality. Montague Island, NSW, Australia, $36^{\circ} 15^{\prime} \mathrm{S}$ $150^{\circ} 14^{\prime} \mathrm{E}, 23 \mathrm{~m}$ depth.

Description. Based on holotype female, $20.5 \mathrm{~mm}$, AM P37165. Head much deeper than long, anterior margin with notch extended into a slit; rostrum absent; eye present, elongate, reniform. Antenna 1 peduncular article 1 not ballshaped proximally, distal margin without a medial spine; peduncular article 2 medium length; flagellum with callynophore, calceoli absent. Antenna 2 flagellum about as long as that of antenna 1, without calceoli. Mouthpart bundle subconical. Epistome/upper lip with broad midanterior bulge (lateral view). Mandible lacinia mobilis a stemmed, distally-cusped blade; accessory setal row with intermediate setae; palp article 2 with 13 posterodistal setae, article 3 without A3-seta. Maxilliped outer plate with distal margin smooth, medial margin without notch.

Gnathopod 1 carpus subequal in length to propodus; propodus, posterior margin without robust setae. Gnathopod 2 palm slightly acute, with 3 lateral robust setae, 2 medial robust setae. Pereopods 3 and 4 merus and carpus without setal fringe. Pereopod 4 coxa with anterior and posterior margins subparallel, anteroventral corner rounded. Pereopods 5-7 with distal articles elongate, dactyls short and stocky. Pereopod 5 basis expanded posteriorly, rounded. Pereopod 7 basis subrectangular, posteroventral corner subquadrate, posteroventral margin straight.

Epimeron 3 posterior margin smooth, with notch slightly above rounded posteroventral corner. Uropod 1 peduncle dorsolateral margin with 14 robust setae; outer ramus without large spines between robust setae. Uropod 2 inner ramus not constricted. Uropod 3 rami lanceolate; without plumose setae; outer ramus 1-articulate. Telson slightly cleft (about 25\%).

Male (sexually dimorphic characters). Based on paratype male, $18.2 \mathrm{~mm}$, AM P37166. Antenna 1 flagellum without callynophore. Antenna 2 flagellum with calceoli. Mandible palp article 2 with 26 posterodistal setae. Gnathopod 2 palm with 2 lateral robust setae.

Etymology. The specific name philatelica alludes to the species' public debut on an Australian two cent postage stamp issued in 1984. (The species was then incorrectly identified as Waldeckia sp.).

Remarks. Amaryllis philatelica appears to be most similar to a small group of species with a smooth posterior margin on epimeron 3 and a subrectangular basis on pereopod 7 (A. macrophthalma, A. philatelica and A. spencerensis). It differs from A. macrophthalma and A. spencerensis in that the notch on the posterior margin of epimeron 3 is only slightly above the posteroventral corner.

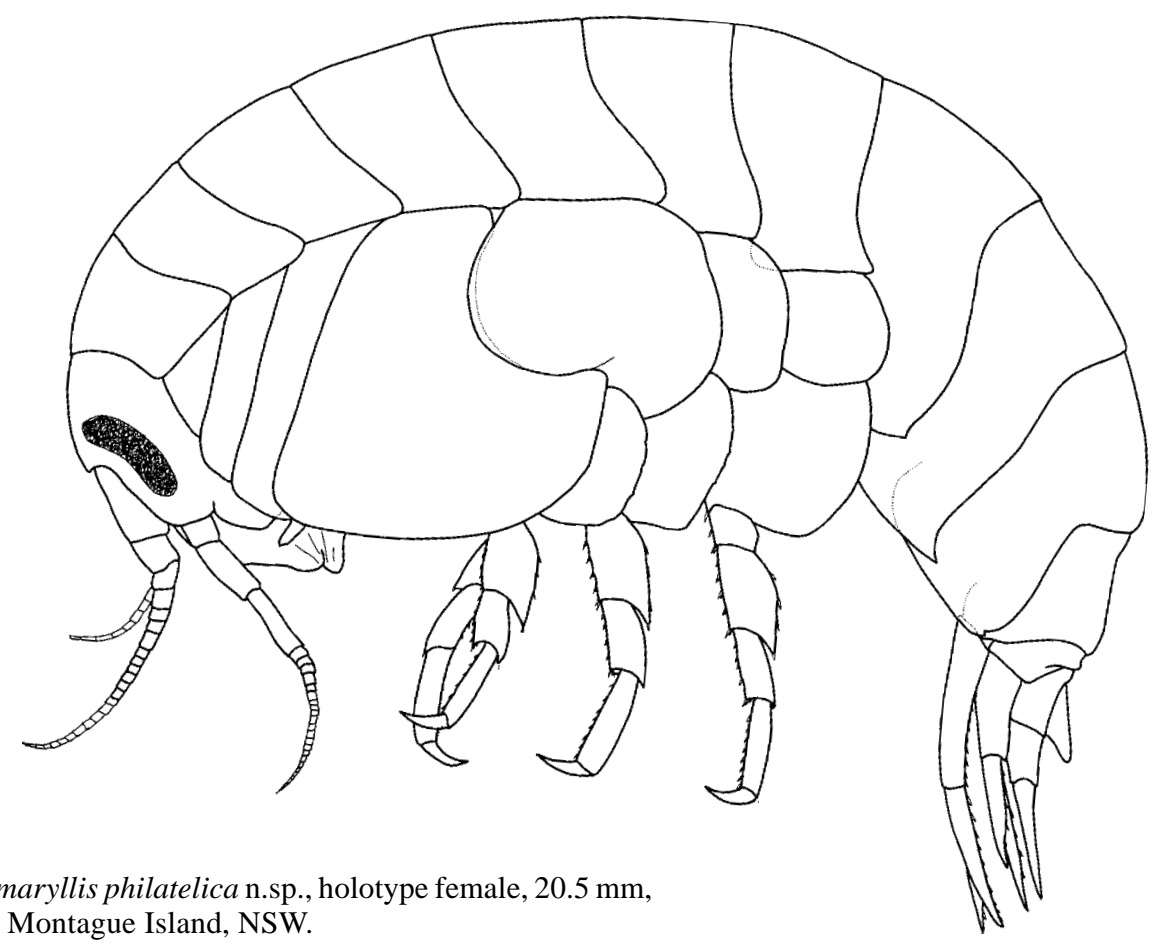

Figure 31. Amaryllis philatelica $\mathrm{n} . \mathrm{sp} .$,
AM P37165, Montague Island, NSW. 


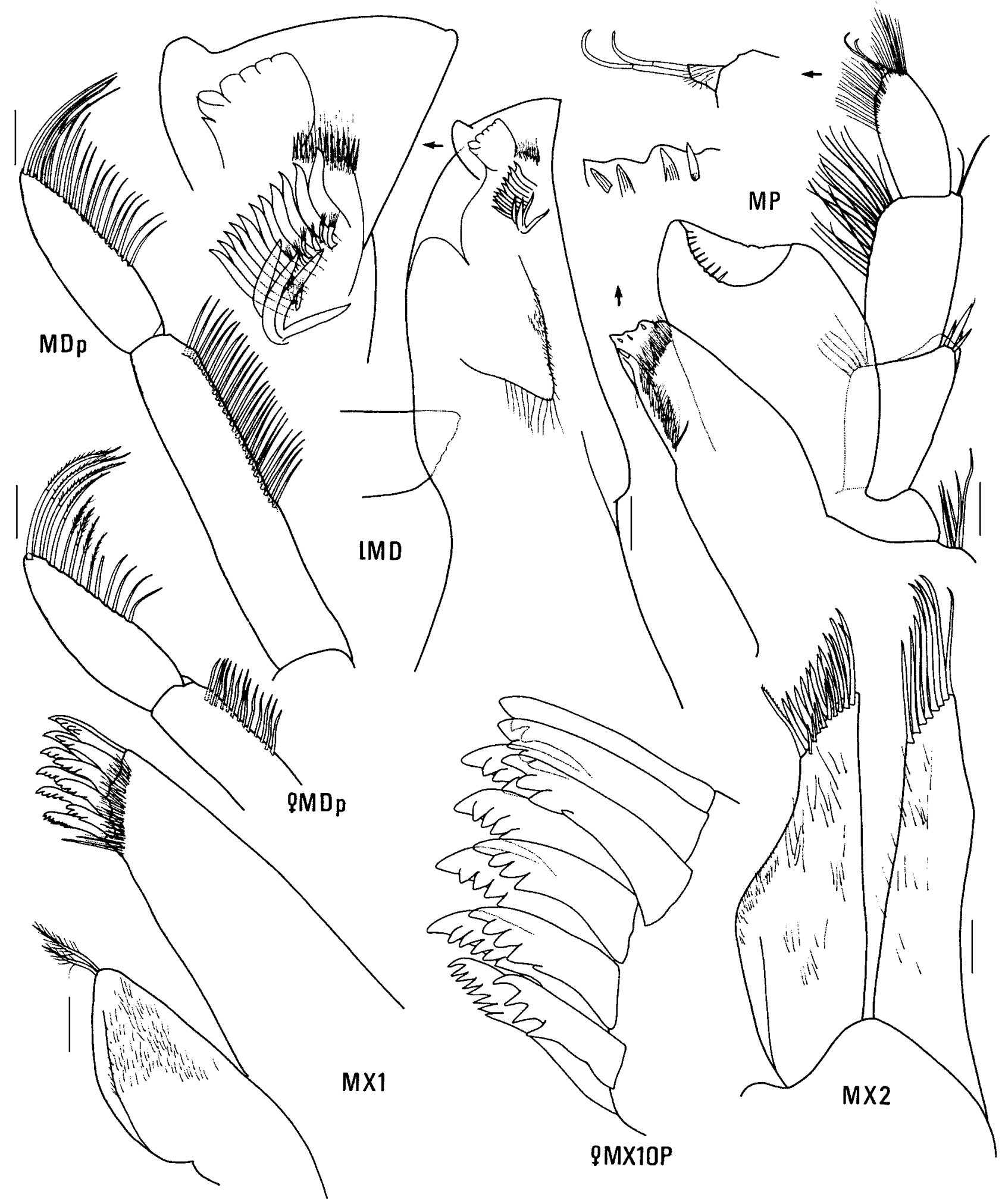

Figure 32. Amaryllis philatelica $\mathrm{n} . \mathrm{sp}$., holotype female, $20.5 \mathrm{~mm}$, AM P37165; paratype male, $18.2 \mathrm{~mm}$, AM P37166; Montague Island, NSW. Scales represent $0.1 \mathrm{~mm}$.

Amaryllis philatelica is often found in association with the soft coral Capnella gaboensis and bryozoans of the genus Triphyllozoon. Judging from its subconical-shaped mouthpart bundle, A. philatelica might feed by nipping off the small polyps of its hosts.

This species has attracted the attention of scuba divers and underwater photographers, partly because of its (relatively) large size but mostly because of its striking colour pattern. The body is a brilliant deep crimson with each segment of the pereon and pleon, the head, each of the coxae and the bases of pereopods 5 to 7 outlined in white; the antennae, protruding mouthparts and distal articles of the pereopods are orange/yellow and there is a single orange/ yellow stripe along the dorsum. Lowry (in Karacsonyi, 1997) commented that the brilliant colour pattern might be a warning to potential predators that $A$. philatelica tastes 


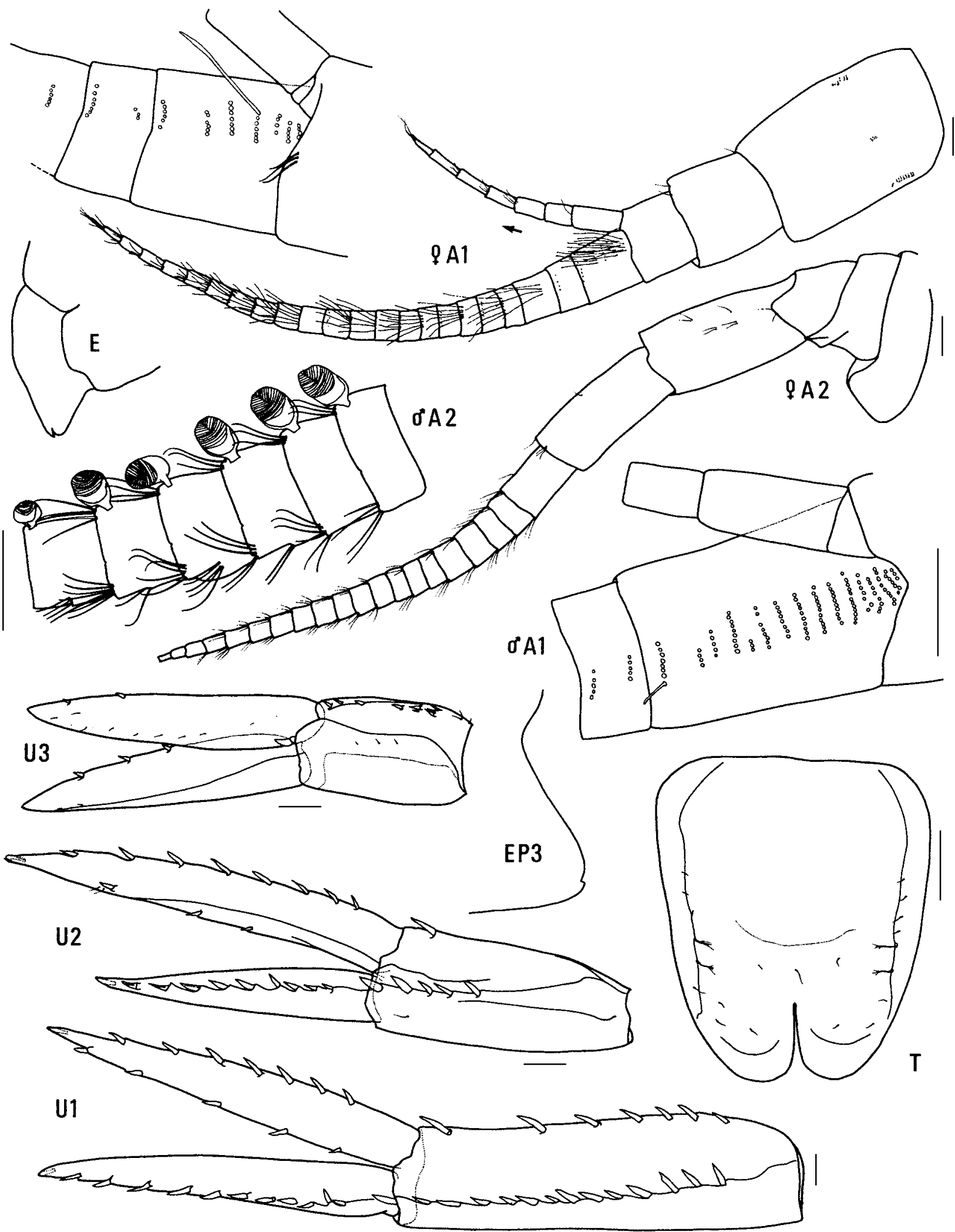

Figure 33. Amaryllis philatelica n.sp., holotype female, $20.5 \mathrm{~mm}$, AM P37165; paratype male, $18.2 \mathrm{~mm}$, AM P37166; Montague Island, NSW. Scales represent $0.2 \mathrm{~mm}$.

unpleasant. The fact that individuals show non-cryptic behaviour - they do not avoid divers and have been known to leave their host and sit on the hand or head of a diver trying to photograph them (Karacsonyi, pers. comm.) - is circumstantial evidence for this theory.
The original colour photographs used to produce the 1984 postage stamp were taken by Rudi Kuiter, at Montague Island. They have been subsequently reproduced on postcards and as an illustration of Amaryllis sp. in Debelius (1999) and Edgar (1997). Some stunning photographs of 


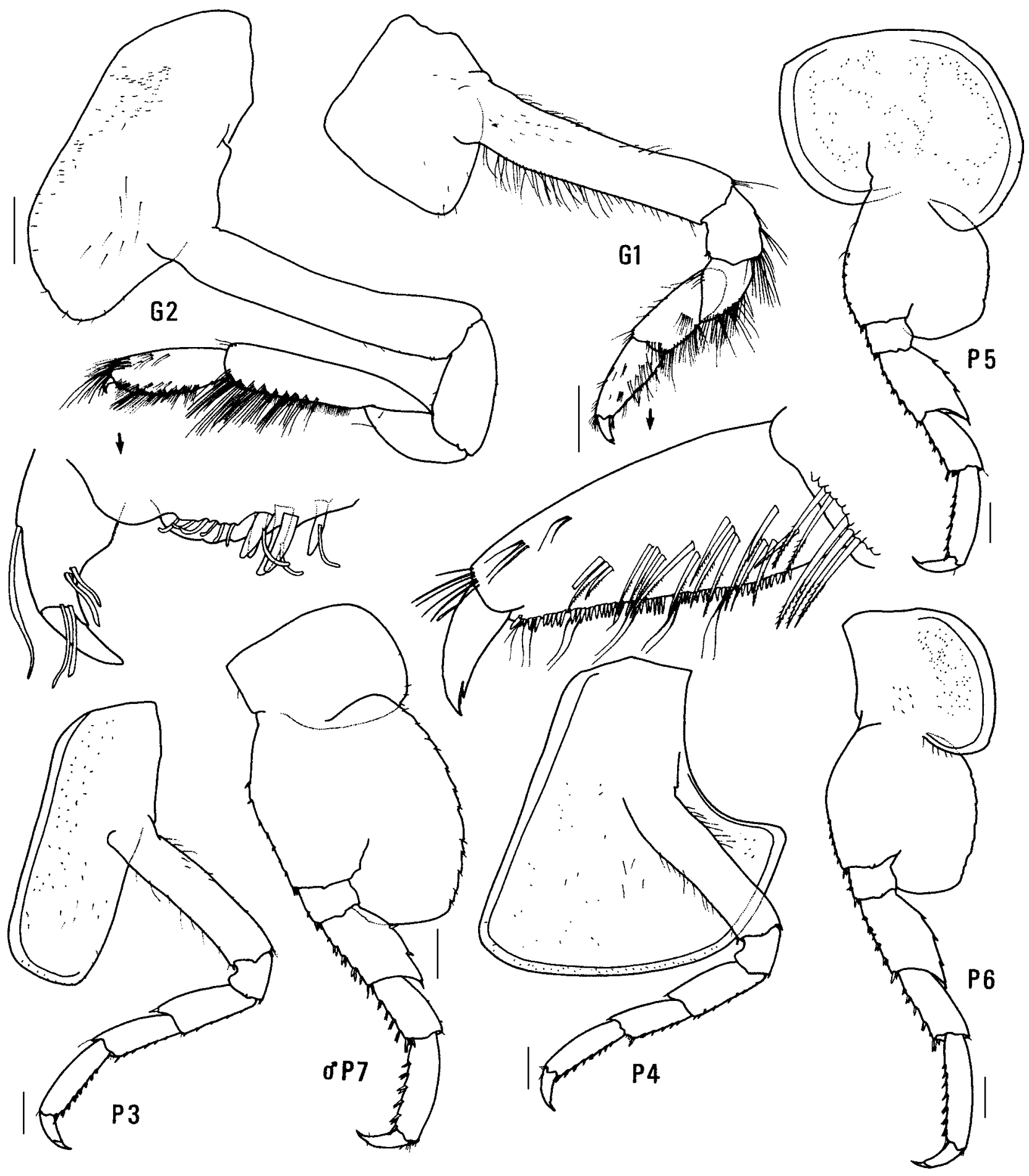

Figure 34. Amaryllis philatelica $\mathrm{n} . \mathrm{sp}$., holotype female, $20.5 \mathrm{~mm}$, AM P37165; paratype male, $18.2 \mathrm{~mm}$, AM P37166; Montague Island, NSW. Scales represent $0.5 \mathrm{~mm}$.

"the red sea flea" were taken by Tony Karacsonyi, near Ulladulla, and published in Australian Geographic (Karacsonyi, 1997) and as a poster released by Sportdiving Magazine.

Habitat. Amaryllis philatelica has been recorded and collected on bryozoans, soft corals and sponges.

Distribution. Southeastern and southern Australia; from Solitary Islands on the east coast to Foul Bay in southwestern Australia; 20-75 m depth.

\section{Amaryllis quokka n.sp.}

Figs. 35-37

Type material. HoLOTYPE, female, $13.4 \mathrm{~mm}$, ovigerous, AM P37373; 1 PARATYPE, male, 8.0 mm, AM P37374; Cathedral Rock, Rottnest Island, Western Australia, eastern Indian Ocean, $32^{\circ} 01.5^{\prime} \mathrm{S} 115^{\circ} 27^{\prime} \mathrm{E}, 3 \mathrm{~m}$, orange tunicates under limestone rock overhangs, J.K. Lowry, 21 December 1983, stn WA-247. 


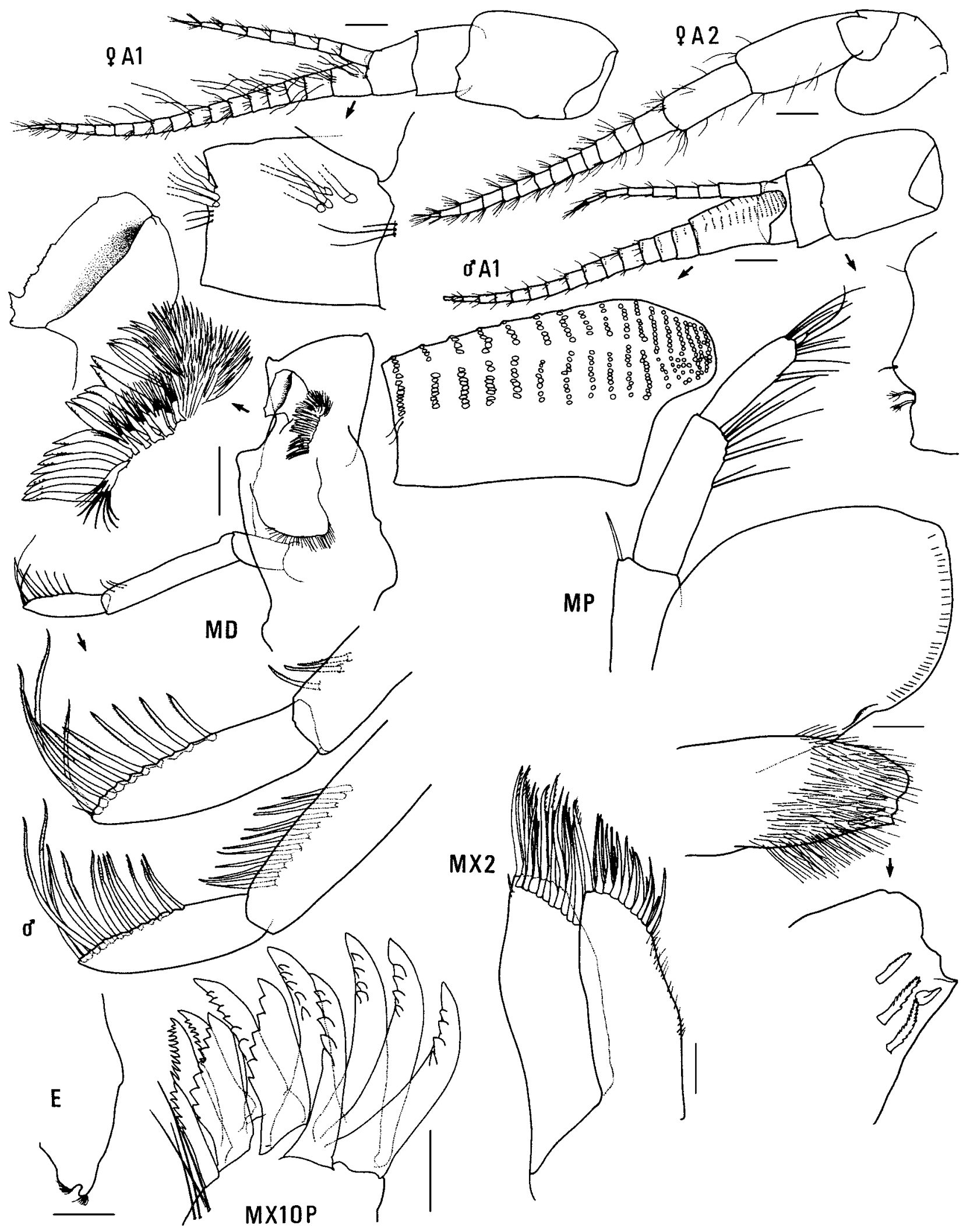

Figure 35. Amaryllis quokka n.sp., holotype female, 13.4 mm, AM P37373; paratype male, 8.0 mm, AM P37374; Rottnest Island, Western Australia. Scales for A1, 2, MD represent $0.2 \mathrm{~mm}$; remainder represent $0.1 \mathrm{~mm}$.

Additional material. SOUTH AUSTRALIA: 10 specimens, AM P57867, Blanche Harbour, Spencer Gulf, [approx. 32 $42^{\prime}$ S 137 $46^{\prime} \mathrm{E}$ ], 9 m, K. Sheard, 8 March 1938, FL Whyalla. 7 specimens, AM P57868, behind Lowly Point, Spencer Gulf, [approx. $33^{\circ} \mathrm{S} 137^{\circ} 46^{\prime} \mathrm{E}$ ], $18 \mathrm{~m}$, K. Sheard, 7 March 1938, FL Whyalla. 1 specimen, SAMA C5985, Dangerous Reef, Spencer Gulf, [approx. $34^{\circ} 49^{\prime} \mathrm{S} 136^{\circ} 12^{\prime} \mathrm{E}$ ], dredged, K. Sheard. 1 specimen, AM P57869, Nepean Bay, Kangaroo Island, 3542'S 137³7'E, dredged, F. Moorhouse, May 1938. 1 specimen, SAMA C5986, Nepean Bay, Kangaroo Island, [approx. $35^{\circ} 42^{\prime} \mathrm{S} 137^{\circ} 37^{\prime} \mathrm{E}$ ], dredged, F. Moorhouse, 7-9 May 1938. 3 specimens, AM P57870, Gulf St Vincent, Fulton, 1902. WeSTERN Australia: 2 specimens, NMV J13980, N side of Cape Riche, $34^{\circ} 37^{\prime} \mathrm{S} 118^{\circ} 47^{\prime} \mathrm{E}, 6 \mathrm{~m}$, sponges on vertical rock face, 


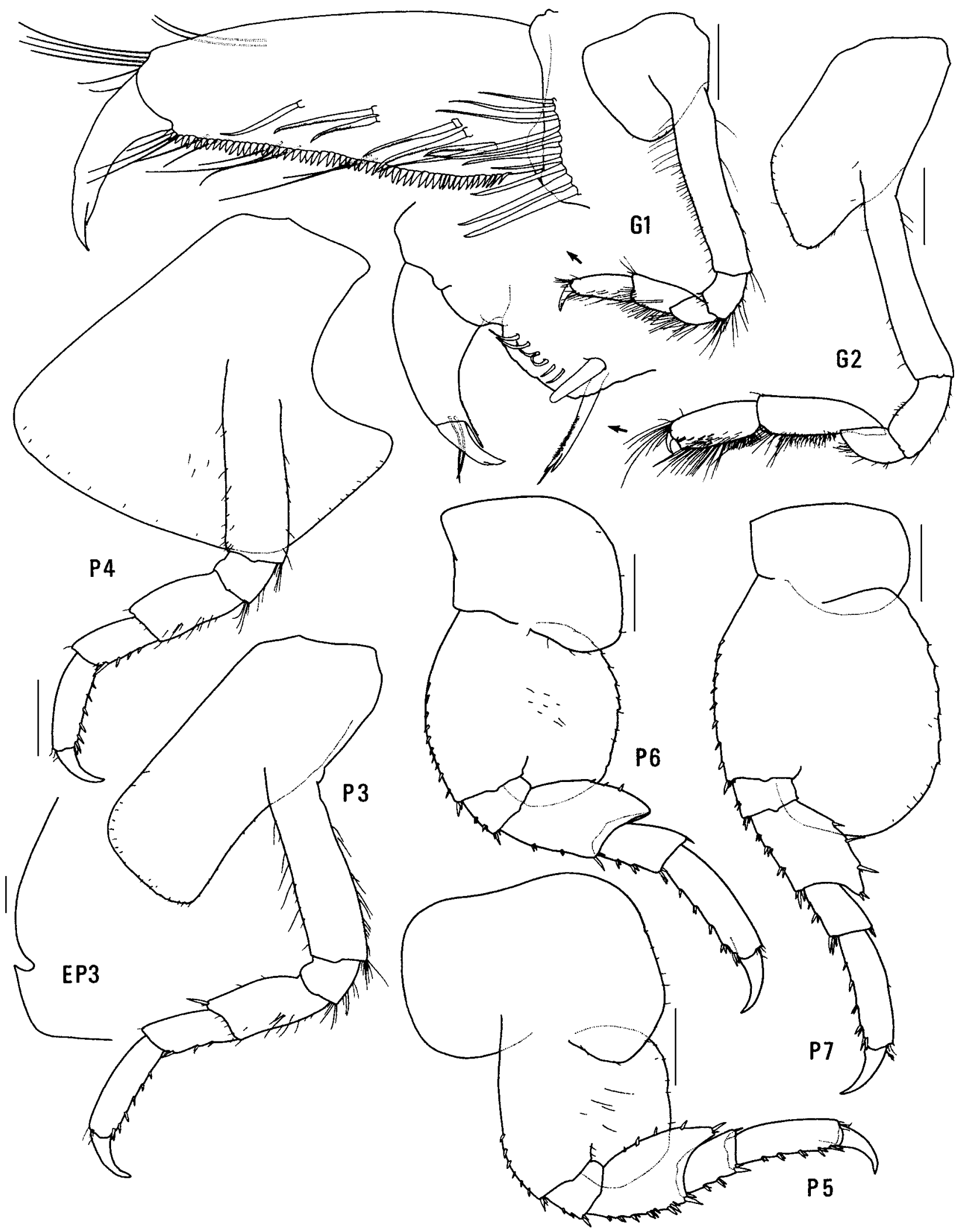

Figure 36. Amaryllis quokka n.sp., holotype female, 13.4 mm, AM P37373, Rottnest Island, Western Australia. Scale for EP3 represents $0.1 \mathrm{~mm}$; remainder represent $0.5 \mathrm{~mm}$.

G.C.B. Poore \& H.M. Lew Ton, 14 April 1985, stn SWA-47. 1 specimen, NMV J13981, N end of Little Beach, Two Peoples Bay, 34 58.4'S $118^{\circ} 11.7^{\prime} \mathrm{E}, 6 \mathrm{~m}$, tufted algae on boulders, G.C.B. Poore \& H.M. Lew Ton, 5 April 1985, stn SWA-11. 1 specimen, AM P37375, Vancouver Peninsula, near Mistaken Island, King George Sound, $35^{\circ} 04^{\prime} \mathrm{S} 117^{\circ} 56^{\prime} \mathrm{E}$, 2 m, sponges, J.K. Lowry, 13 December 1983, stn WA-103. 10 specimens,
AM P37376, off Possession Point, King George Sound, $35^{\circ} 02^{\prime} \mathrm{S} 117^{\circ} 55^{\prime} \mathrm{E}$, $7 \mathrm{~m}$, brown algae, bryozoans and sponge, R. Springthorpe \& J.K. Lowry, 14 December 1983, stn WA-133. 9 specimens, AM P37377, same locality, 7 m, mixed sponges and algae, J.K. Lowry, 14 December 1983, stn WA-135.

Type locality. Cathedral Rock, Rottnest Island, Western 


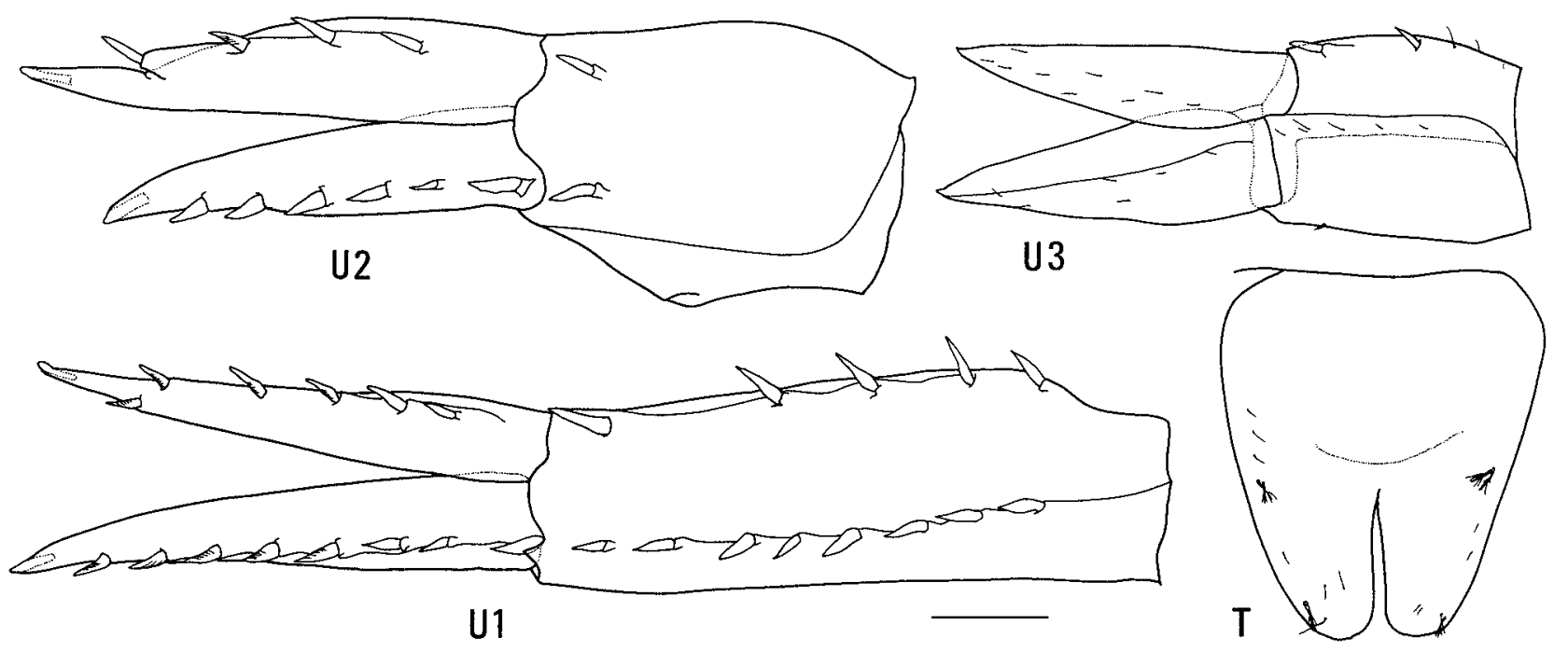

Figure 37. Amaryllis quokka n.sp., holotype female, 13.4 mm, AM P37373, Rottnest Island, Western Australia. Scale represents $0.2 \mathrm{~mm}$.

Australia, eastern Indian Ocean, $32^{\circ} 01.5^{\prime} \mathrm{S} 115^{\circ} 27^{\prime} \mathrm{E}, 3 \mathrm{~m}$ depth.

Description. Based on holotype female, $13.4 \mathrm{~mm}$, AM P37373. Head much deeper than long, anterior margin with notch extended into a slit; rostrum absent; eye present, elongate, reniform. Antenna 1 peduncular article 1 not ballshaped proximally, distal margin with small medial spine; peduncular article 2 medium length; flagellum with callynophore (vestigial), calceoli absent. Antenna 2 flagellum about as long as that of antenna 1, without calceoli. Mouthpart bundle subconical. Epistome/upper lip with broad mid-anterior bulge (lateral view). Mandible lacinia mobilis a stemmed, cup-like blade; accessory setal row with intermediate setae; palp article 2 with 3 posterodistal setae, article 3 without A3-seta. Maxilliped outer plate with distal margin smooth, medial margin without notch.

Gnathopod 1 carpus subequal in length to propodus; propodus, posterior margin without robust setae. Gnathopod 2 palm transverse, without lateral robust setae, with 1 medial robust seta. Pereopods 3 and 4 merus and carpus without setal fringe. Pereopod 4 coxa with anterior and posterior margins subparallel, anteroventral corner rounded. Pereopods 5-7 with distal articles elongate, dactyls short and stocky. Pereopod 5 basis expanded posteriorly, rounded. Pereopod 7 basis rounded posteriorly, posteroventral corner rounded, posteroventral margin curved.
Epimeron 3 posterior margin smooth, with notch well above rounded posteroventral corner. Uropod 1 peduncle dorsolateral margin with 9 robust setae; outer ramus without large spines between robust setae. Uropod 2 inner ramus slightly constricted. Uropod 3 rami lanceolate; without plumose setae; outer ramus 1-articulate. Telson moderately cleft (about 46\%).

Male (sexually dimorphic characters). Based on paratype male, $8.0 \mathrm{~mm}$, AM P37374. Antenna 1 flagellum with callynophore. Mandible palp article 2 with 11 posterodistal setae.

Etymology. The species name quokka is a reference to the small marsupials that inhabit Rottnest Island.

Remarks. Amaryllis quokka belongs to the group of species with a smooth posterior margin on epimeron 3 and a rounded posteroventral corner on the basis of pereopod 7 (A. brevicornis, A. kamata, A. moona, A. olinda). Within this group it differs from all other species in the transverse palm of gnathopod 2.

Habitat. Amaryllis quokka has been collected on sedentary invertebrates, most often sponges.

Distribution. Southern and southwestern Australia, from the South Australian Gulfs to Rottnest Island; 3-18 m depth. 


\section{Amaryllis spencerensis n.sp.}

Figs. 38-40

Type material. HoLOTYPE, female, $16.0 \mathrm{~mm}$, ovigerous (15 eggs), AM P37096; 3 PARATYPES, female, AM P37097; $19 \mathrm{~km}$ from Mount Young, toward Wallaroo, Spencer Gulf, South Australia, [approx. $33^{\circ} 18^{\prime} \mathrm{S} 137^{\circ} 31^{\prime} \mathrm{E}$ ], $18 \mathrm{~m}, \mathrm{~K}$. Sheard on FL Whyalla, 8 March 1938. 1 PARATYPE, male, 11.0 mm, AM P37098, Western Shoal, Spencer Gulf, South Australia, [approx. $33^{\circ} 09^{\prime} \mathrm{S} 137^{\circ} 31^{\prime} \mathrm{E}$ ], $9 \mathrm{~m}$, K. Sheard on FL Whyalla, 10 March 1938. 2 PARATYPES, female, SAMA C5987, Dangerous Reef, Spencer Gulf, South Australia, [approx. $34^{\circ} 49^{\prime} S 136^{\circ} 12^{\prime} E$ ], dredged, K. Sheard.

Additional material. Three females, AM P37099, unknown locality in Spencer Gulf and/or Gulf St Vincent, South Australia.
Type locality. $19 \mathrm{~km}$ from Mount Young, toward Wallaroo, Spencer Gulf, South Australia, [approx. $33^{\circ} 18^{\prime} \mathrm{S} 137^{\circ} 31^{\prime} \mathrm{E}$ ], $18 \mathrm{~m}$ depth.

Description. Based on holotype female, $16.0 \mathrm{~mm}$, AM P37096. Head much deeper than long, anterior margin with notch extended into a slit; rostrum absent; eye present, elongate, reniform. Antenna 1 peduncular article 1 not ballshaped proximally, distal margin with well-developed medial spine; peduncular article 2 medium length; flagellum without callynophore, calceoli absent. Antenna 2 flagellum about as long as that of antenna 1, without calceoli. Mouthpart bundle subconical. Epistome/upper lip with strong mid-anterior angle (lateral view). Mandible lacinia mobilis a stemmed, distally-cusped blade; accessory setal row with intermediate setae; palp article 2 with 2

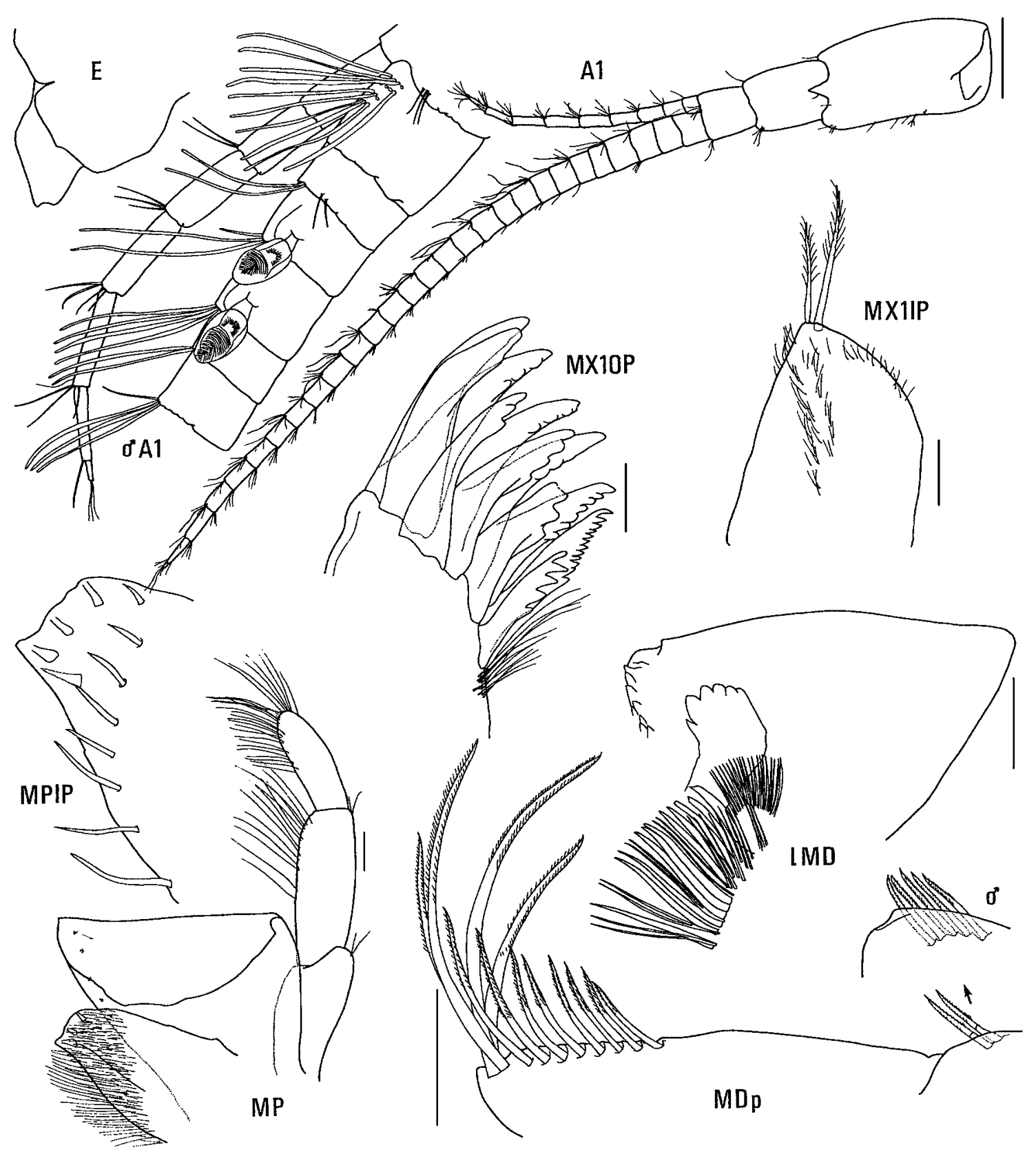

Figure 38. Amaryllis spencerensis n.sp., holotype female, 16.0 mm, AM P37096; paratype male, 11.0 mm, AM P37098; Spencer Gulf, South Australia. Scale for A1 represents $0.5 \mathrm{~mm}$; remainder represent $0.1 \mathrm{~mm}$. 


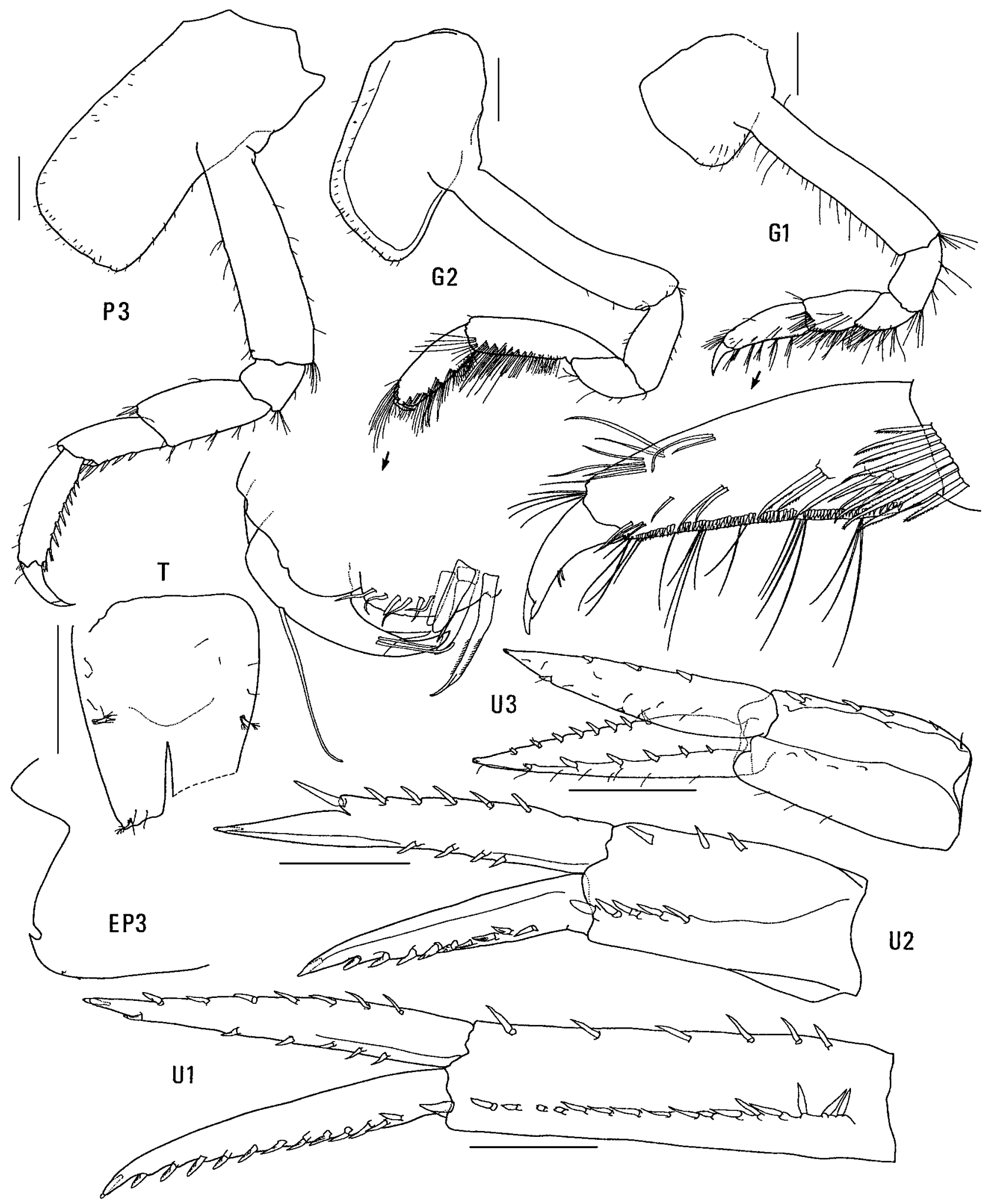

Figure 39. Amaryllis spencerensis n.sp., holotype female, 16.0 mm, AM P37096, Spencer Gulf, South Australia. Scales represent $0.5 \mathrm{~mm}$.

posterodistal setae, article 3 without A3-seta. Maxilliped outer plate with distal margin smooth, medial margin without notch.

Gnathopod 1 carpus subequal in length to propodus $(0.9 \times)$; propodus, posterior margin without robust setae. Gnathopod 2 palm slightly acute, with 2 lateral robust setae, 1 medial robust seta. Pereopods 3 and 4 merus and carpus without setal fringe. Pereopod 4 coxa with anterior and posterior margins subparallel, anteroventral corner rounded. Pereopods 5-7 with distal articles elongate, dactyls short and stocky. Pereopod 5 basis expanded posteriorly, rounded. Pereopod 7 basis subrectangular, posteroventral corner notched, posteroventral margin straight.

Epimeron 3 posterior margin smooth, with notch well above rounded posteroventral corner. Uropod 1 peduncle 


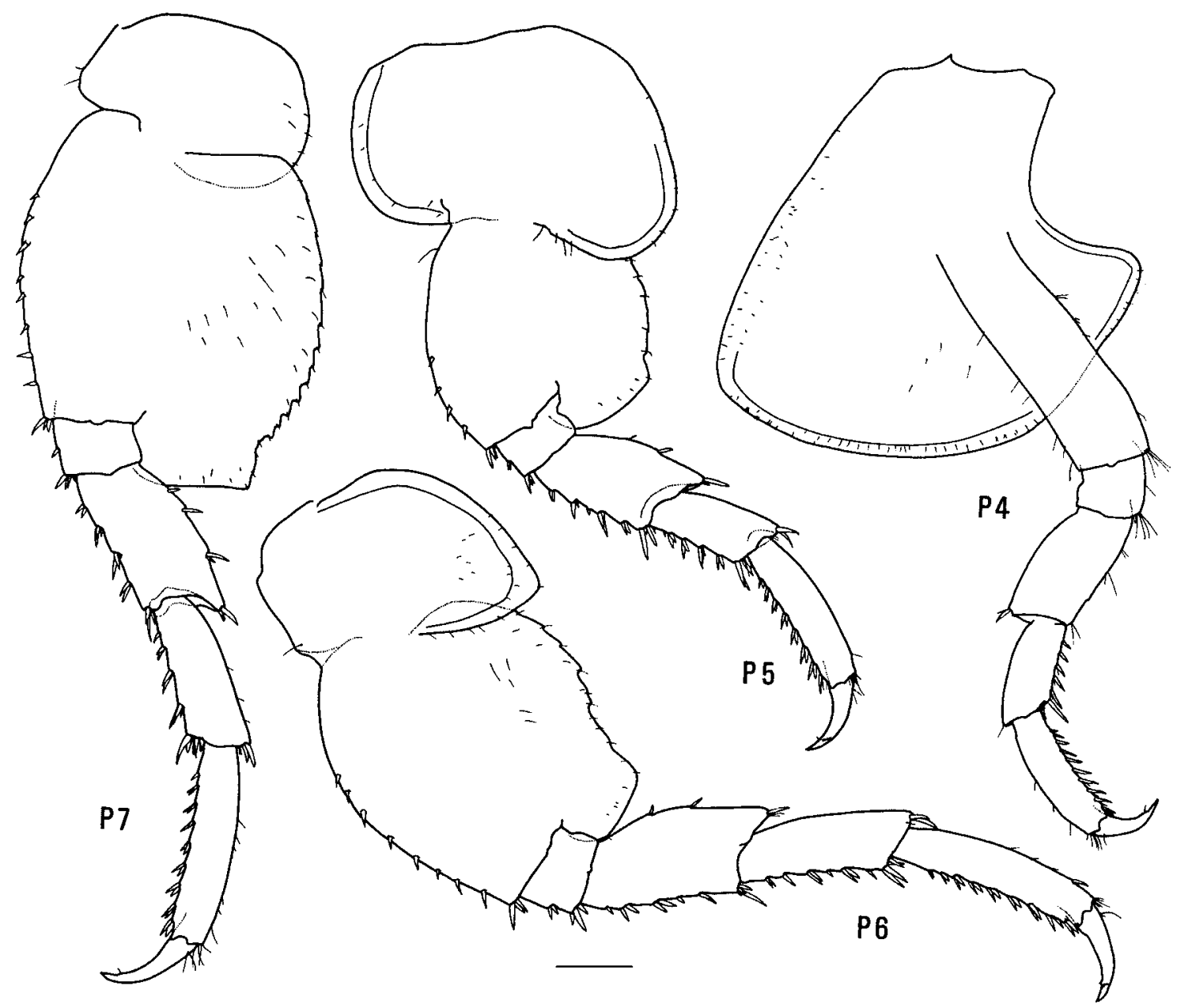

Figure 40. Amaryllis spencerensis n.sp., holotype female, 16.0 mm, AM P37096, Spencer Gulf, South Australia. Scale represents $0.5 \mathrm{~mm}$.

dorsolateral margin with 17 robust setae; outer ramus without large spines between robust setae. Uropod 2 inner ramus slightly constricted. Uropod 3 rami lanceolate; without plumose setae; outer ramus 1-articulate. Telson moderately cleft (about 37\%).

Male (sexually dimorphic characters). Based on paratype male, $11 \mathrm{~mm}$, AM P37098. Antenna 1 flagellum with callynophore, with calceoli. Antenna 2 flagellum with calceoli. Mandible palp article 2 with 5 posterodistal setae. Gnathopod 2 palm with 1 lateral robust seta.

Etymology. Named for the type locality, Spencer Gulf.

Remarks. Amaryllis spencerensis belongs to a small group of species with a smooth posterior margin on epimeron 3 and a subrectangular basis on pereopod 7 (A. macrophthalma, A. philatelica and $A$. spencerensis). Within this group $A$. macrophthalma and $A$. spencerensis both have a welldeveloped medial spine on peduncular article 1 of antenna 1 and a notch above the rounded posteroventral corner on epimeron 3. In A. spencerensis the posteroventral corner of the basis of pereopod 7 is notched, in contrast to subquadrate in A. macrophthalma, and there are significantly less robust setae defining the palm of gnathopod 2 in the female.

Habitat. Not known.

Distribution. Known only from Spencer Gulf, South Australia; 9-18 m depth.

\section{Bamarooka n.gen.}

Diagnosis. Mouthpart bundle subconical. Mandible palp article 3 without A3-seta. Pereopod 4 coxa with anterior margin slightly obtuse, posterior margin rounded. Pereopods 5-7 with distal articles elongate. Uropod 3 rami lanceolate; without plumose setae in male and female; outer ramus 1articulate.

Type species. Amaryllis bathycephala Stebbing, 1888.

Species composition. Bamarooka contains six species: $B$. anomala n.sp.; B. bathycephala (Stebbing, 1888); $B$. dinjerra n.sp.; B. endota n.sp.; B. kimbla n.sp. and $B$. tropicalis n.sp.

Etymology. Bamarooka is an Australian Aboriginal word meaning "oval shield", an allusion to coxa 4.

Remarks. Species of Bamarooka are mainly tropical amphipods distinguished from other amaryllidines by their strangely shaped coxa 4 . Species level variation in Bamarooka is high, particularly in the eyes and the basis of pereopods 5 to 7. Bamarooka also occurs outside Australia, in New Caledonia (unpublished records) (see Fig. 41).

Distribution. Northwestern, northern and eastern coasts of Australia; New Caledonia; 2-175 m depth. 


\section{Key to species of Bamarooka}

1 Head without rostrum; eye reniform ....................................................................... 2

Head with rostrum; eye ventrally tapered ....................................................................... 3

2 Antenna 1 peduncular article 1 distal margin with small medial spine. Pereopod 5 basis expanded posteriorly, not linear

Antenna 1 peduncular article 1 distal margin without medial spine.

Pereopod 5 basis not expanded, linear

B. anomala

3 Pereopod 5 basis not pear-shaped. Female gnathopod 2 palm without lateral robust setae

Pereopod 5 basis pear-shaped (expanded posterodistally). Female gnathopod 2 palm with 1-4 lateral robust setae

B. bathycephala

4 Pereopod 5 basis with proximal posterior shoulder sloped 5

Pereopod 5 basis almost round

B. endota

5 Head with rostrum rounded. Female gnathopod 2 palm with 1 medial robust seta

B. dinjerra

Head with rostrum blunt cone-shaped. Female gnathopod 2 palm with 3 or 4 medial robust setae

B. kimbla

\section{Bamarooka anomala n.sp.}

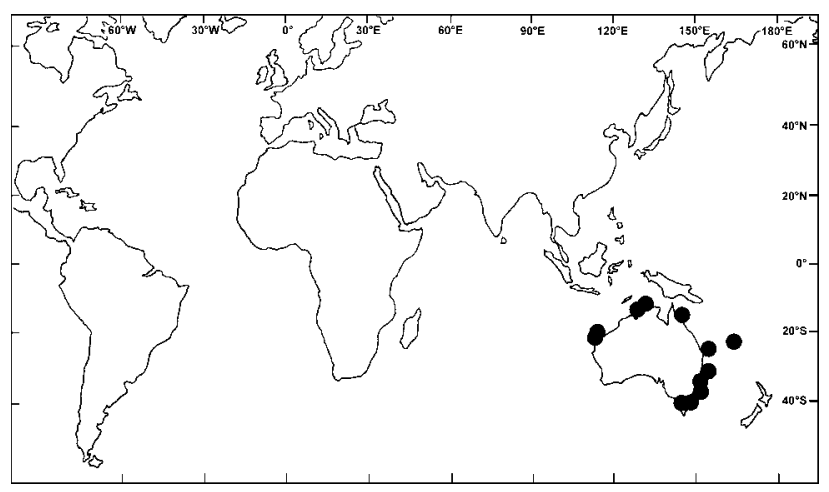

Figure 41. Distribution of the genus Bamarooka.
Figs. $42-44$

Type material. HolOTYPE, female, $10.2 \mathrm{~mm}$, with 15 embryos, AM P36878; 1 PARATYPE, male, $6.5 \mathrm{~mm}$, AM P36879; North West Shelf, NW of Port Hedland, Western Australia, eastern Indian Ocean, $19^{\circ} 28.3^{\prime} \mathrm{S} 118^{\circ} 55.2^{\prime} \mathrm{E}$ to $19^{\circ} 28.4^{\prime} \mathrm{S} 118^{\circ} 55.6^{\prime} \mathrm{E}, 38-39 \mathrm{~m}$, epibenthic sled, 31 August 1983, FRV Soela, CSIRO NWS stn 04.B9.S. 1 PARATYPE, female, AM P36880, same locality, 1954.6'S $117^{\circ} 56.0^{\prime} \mathrm{E}$ to $19^{\circ} 55.1^{\prime} \mathrm{S} 117^{\circ} 55.6^{\prime} \mathrm{E}, 44 \mathrm{~m}$, epibenthic sled, 18 February 1983, FRV Soela, CSIRO NWS stn 01.B3.S. 1 PARATYPE, AM P36881, same locality, $19^{\circ} 28.6^{\prime} \mathrm{S} 118^{\circ} 55.0^{\prime} \mathrm{E}$ to $19^{\circ} 28.2^{\prime} \mathrm{S} 118^{\circ} 55.3^{\prime} \mathrm{E}, 39 \mathrm{~m}$, epibenthic sled, 28 June 1983, FRV Soela, CSIRO NWS stn 03.B9.S. 1 PARATYPE, AM $\mathrm{P} 36882$, same locality, $19^{\circ} 29.6^{\prime} \mathrm{S} 118^{\circ} 52.2^{\prime} \mathrm{E}$ to $19^{\circ} 29.4^{\prime} \mathrm{S}$

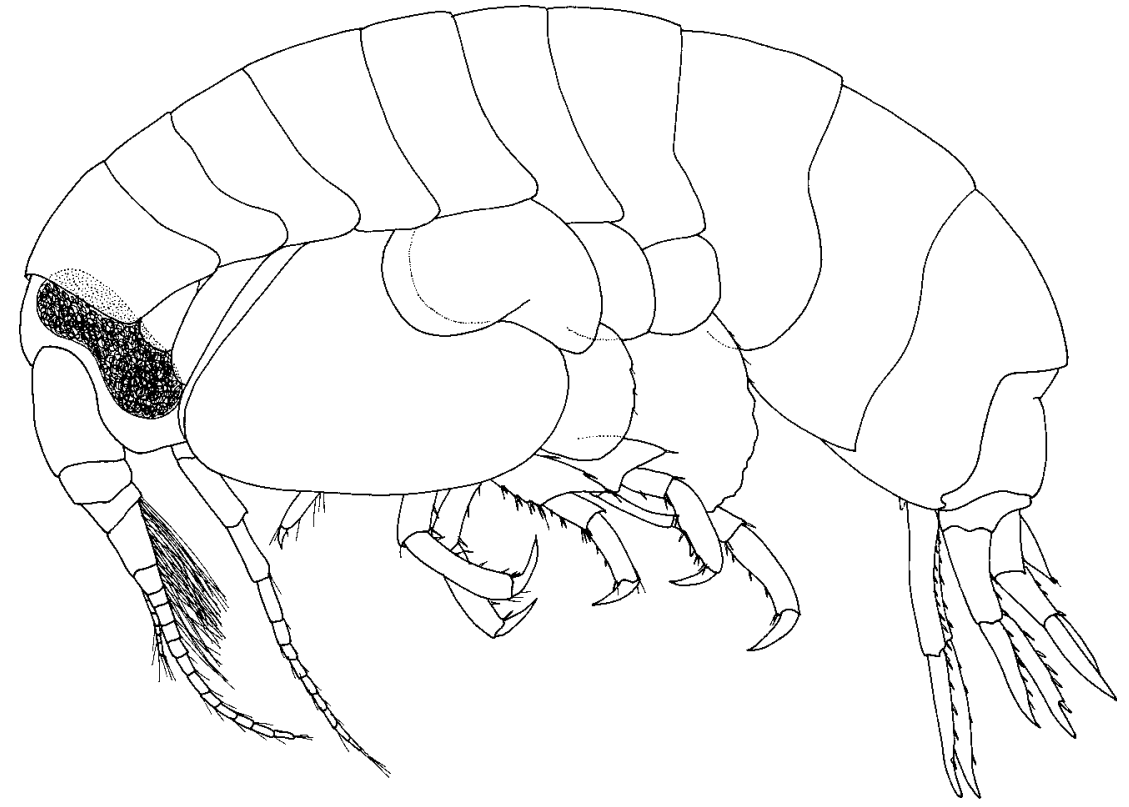

Figure 42. Bamarooka anomala n.sp., paratype male, 6.5 mm, AM P36879, North West Shelf, Western Australia. 


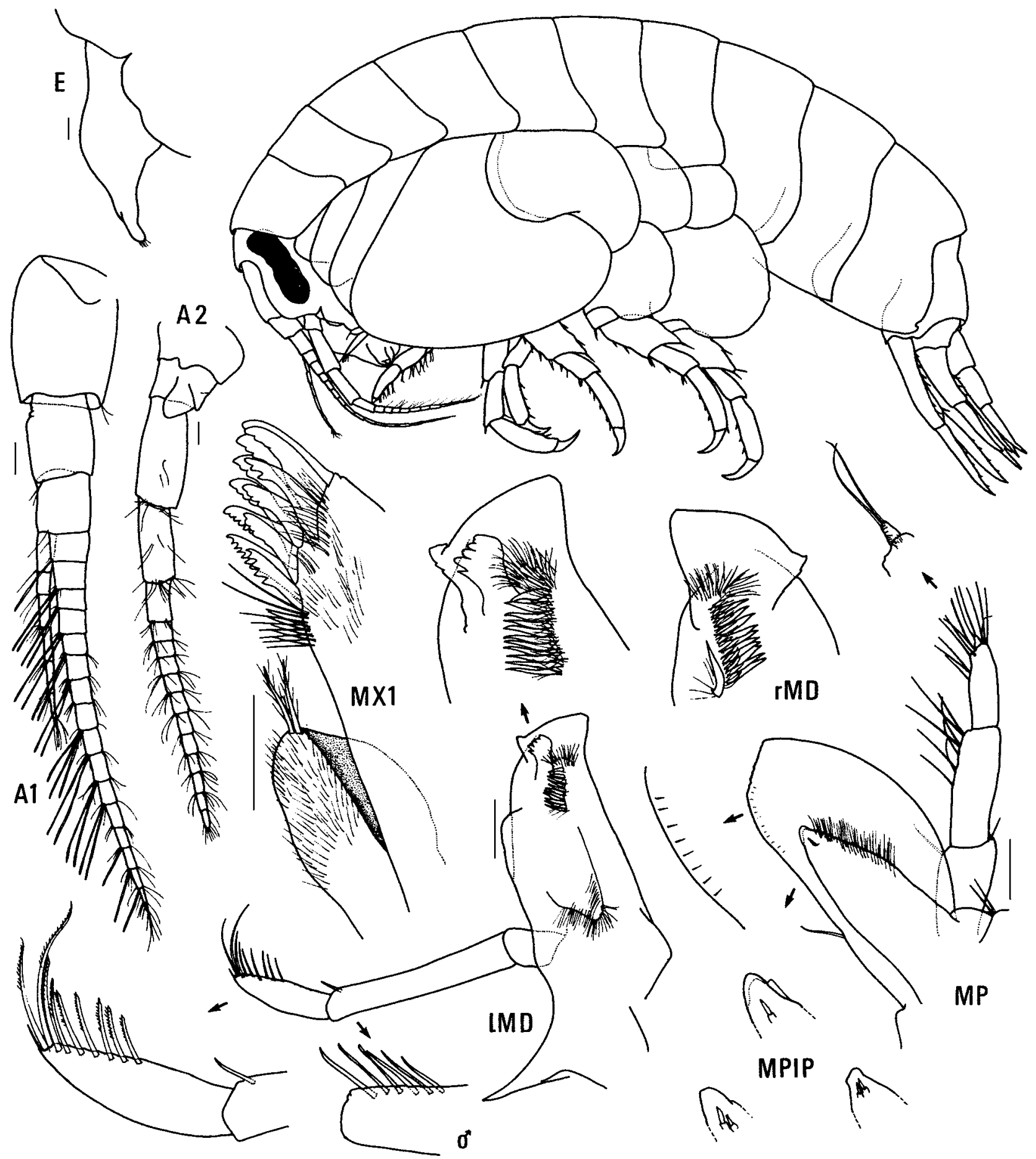

Figure 43. Bamarooka anomala n.sp., holotype female, $10.2 \mathrm{~mm}$, AM P36878; paratype male, $6.5 \mathrm{~mm}$, AM P36879; North West Shelf, Western Australia. Scales represent $0.1 \mathrm{~mm}$.

$118^{\circ} 52.8^{\prime} \mathrm{E}, 38 \mathrm{~m}$, epibenthic sled, 30 August 1983, FRV Soela, CSIRO NWS stn 04.B8.S. 1 PARATYPE, female, AM $\mathrm{P} 36883$, same locality, $19^{\circ} 29.6^{\prime} \mathrm{S} 118^{\circ} 52.2^{\prime} \mathrm{E}$ to $19^{\circ} 29.6^{\prime} \mathrm{S}$ $118^{\circ} 51.4^{\prime} \mathrm{E}, 36-38 \mathrm{~m}$, epibenthic sled, 25 October 1983, FRV Soela, CSIRO NWS stn 05.D4.S. 1 PARATYPE, female, AM $\mathrm{P} 36884$, same locality, $1^{\circ} 29.6^{\prime} \mathrm{S} 118^{\circ} 51.7^{\prime} \mathrm{E}$ to $19^{\circ} 29.9^{\prime} \mathrm{S}$ $118^{\circ} 51.0^{\prime} \mathrm{E}, 40-41 \mathrm{~m}$, epibenthic sled, 25 October 1983, FRV Soela, CSIRO NWS stn 05.D7.S. 2 PARATYPES, male and juvenile, AM P36885, same locality, $19^{\circ} 29.4^{\prime} \mathrm{S} 118^{\circ} 52.5^{\prime} \mathrm{E}$ to $19^{\circ} 30.0^{\prime} \mathrm{S} 118^{\circ} 51.6^{\prime} \mathrm{E}, 38 \mathrm{~m}$, epibenthic sled, 25 October 1983 , FRV Soela, CSIRO NWS stn 05.D8.S. 1 PARATYPE, female,
AM P36886, same locality, $1^{\circ} 27.2^{\prime} \mathrm{S} 118^{\circ} 58.6^{\prime} \mathrm{E}$ to $19^{\circ} 26.7^{\prime} \mathrm{S}$ $118^{\circ} 58.3^{\prime} \mathrm{E}, 36-46 \mathrm{~m}$, epibenthic sled, 8 December 1982, FRV Soela, CSIRO NWS stn 06.B9.S. 1 PARATYPE, female, NMV J7665, North West Shelf, between Port Hedland and Dampier, Western Australia, 19³7.00'S 118 53.00'E, 30-31 m, coarse shell, dredge, 3 June 1983, G.C.B. Poore \& H.M. Lew Ton, FRV Soela, NMV stn NWA-14. 1 PARATYPE, NMV J13935, North West Shelf, between Port Hedland and Dampier, Western Australia, $19^{\circ} 39.00^{\prime} \mathrm{S} 116^{\circ} 31.00^{\prime} \mathrm{E}$ to $19^{\circ} 39.00^{\prime} \mathrm{S}$ $116^{\circ} 43.00^{\prime} \mathrm{E}, 46 \mathrm{~m}$, bryozoans, dredge, 7 June 1983, G.C.B. Poore \& H.M. Lew Ton, FRV Soela, NMV stn NWA-33. 

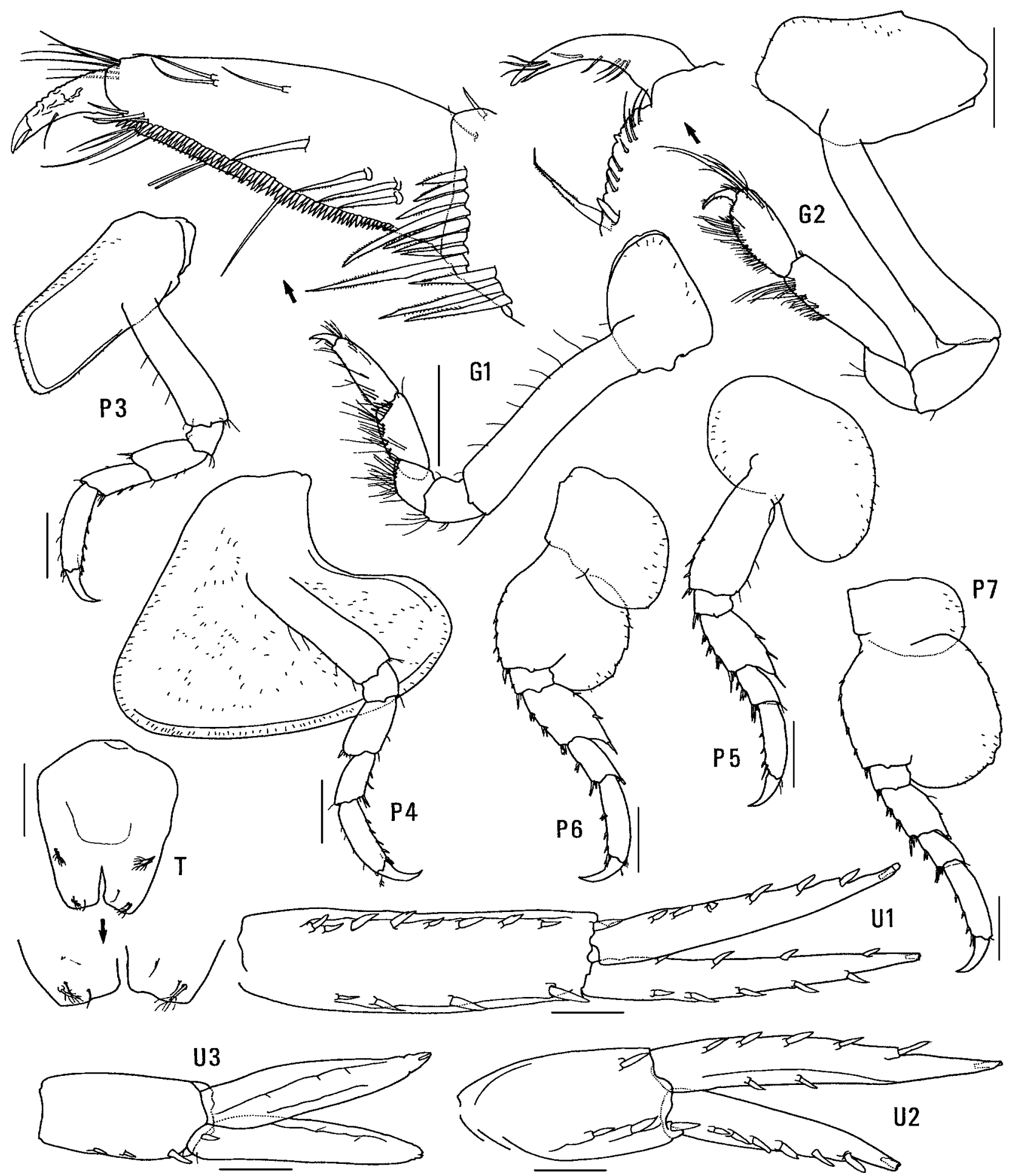

Figure 44. Bamarooka anomala n.sp., holotype female, $10.2 \mathrm{~mm}$, AM P36878; North West Shelf, Western Australia. Scales for U1-3, T represent $0.2 \mathrm{~mm}$; remainder represent $0.5 \mathrm{~mm}$.

Additional material. WeSTERn AUSTRALIA: 4 specimens, AM P36887, inshore limestone reef, North West Cape, $22^{\circ} 00^{\prime} \mathrm{S} 113^{\circ} 55^{\prime} \mathrm{E}, 2 \mathrm{~m}$, coralline alga Halimeda, R. Springthorpe, 31 December 1983, stn WA-329. NORTHERN TERRITORY: 1 specimen, AM P58074, patch reef, north side of New Year Island, Arafura Sea, $10^{\circ} 54^{\prime} \mathrm{S} 133^{\circ} 02^{\prime} \mathrm{E}, 10 \mathrm{~m}$, hydroids on coral, G.C.B. Poore, 14 October 1982, stn NT-17. 1 specimen, AM $\mathrm{P} 36888$, coral patch reefs at NW end of McCluer Island, $11^{\circ} 02^{\prime} \mathrm{S} 132^{\circ} 58^{\prime} \mathrm{E}$, 8 m, sponges, J.K. Lowry, 16 October 1982, stn NT-42.

Type locality. North West Shelf, northwest of Port Hedland, Western Australia, eastern Indian Ocean, $19^{\circ} 28.3^{\prime} \mathrm{S}$ $118^{\circ} 55.2^{\prime} \mathrm{E}$ to $19^{\circ} 28.4^{\prime} \mathrm{S} 118^{\circ} 55.6^{\prime} \mathrm{E}, 38-39 \mathrm{~m}$ depth.

Description. Based on holotype female, $10.2 \mathrm{~mm}$, AM P36878. Head much deeper than long, anterior margin with notch extended into a slit; rostrum absent; eye present, elongate, reniform. Antenna 1 peduncular article 1 not ballshaped proximally, distal margin without a medial spine; peduncular article 2 medium length; flagellum without callynophore, calceoli absent. Antenna 2 flagellum about as long as that of antenna 1, without calceoli. Mouthpart 
bundle subconical. Epistome/upper lip with strong midanterior angle (lateral view). Mandible lacinia mobilis a stemmed, distally-cusped blade; accessory setal row with intermediate setae; palp article 2 with 1 posterodistal seta, article 3 without A3-seta. Maxilliped outer plate with distal margin smooth, medial margin without notch.

Gnathopod 1 carpus subequal in length to propodus; propodus, posterior margin without robust setae. Gnathopod 2 palm slightly acute, with no lateral robust setae, 1 medial robust seta. Pereopods 3 and 4 merus and carpus without setal fringe. Pereopod 4 coxa with anterior margin slightly obtuse, posterior margin rounded, anteroventral corner rounded. Pereopods 5-7 with distal articles elongate, dactyli short and stocky. Pereopod 5 basis not expanded posteriorly (linear). Pereopod 7 basis rounded posteriorly, posteroventral corner rounded, posteroventral margin curved.

Epimeron 3 posterior margin smooth, with notch slightly above rounded posteroventral corner. Uropod 1 peduncle dorsolateral margin with 9 robust setae; outer ramus without large spines between robust setae. Uropod 2 inner ramus slightly constricted. Uropod 3 rami lanceolate, without plumose setae; outer ramus 1-articulate. Telson slightly cleft (about 22\%).

Male (sexually dimorphic characters). Based on paratype male, $6.5 \mathrm{~mm}$, AM P36879 (possibly not fully mature). Antenna 1 flagellum with callynophore. Antenna 2 flagellum without calceoli. Mandible palp article 2 with 6 posterodistal setae.

Etymology. The name reflects the unusual set of characters which defines this species within the genus Bamarooka.
Remarks. Two species of Bamarooka (B. anomala and B. tropicalis) have elongate, reniform eyes. They are easily distinguished from each other by their fifth pereopods, which have a linear basis in $B$. anomala and an expanded basis in B. tropicalis.

Habitat. Bamarooka anomala has been collected from coralline algae, bryozoans, sponges, hydroids and coarse shell sediment but its habitat is not specifically known.

Distribution. Northwestern and northern Australia; 30-46 m depth on North West Shelf and 2-10 m depth at inshore sites.

\section{Bamarooka bathycephala (Stebbing)}

Figs. 45-47

Amaryllis bathycephalus Stebbing, 1888: 699, pl. 27.

Amaryllis bathycephala.-Stebbing, 1906: 24.-Stebbing, 1910a: 633 (list).-Sheard, 1937: 18 (list).-Thurston \& Allen, 1969: 354.

Amaryllis macrophthalmus.-Della Valle, 1893: 781 (in part).

Material examined. NEW South WALES: 1 specimen, AM P58124, between Lion Island and Barrenjoey Head, Broken Bay, [approx. 33 $33^{\circ} \mathrm{S}$ $151^{\circ} 22^{\prime} \mathrm{E}$ ], $25 \mathrm{~m}$, SPCC/UTS, 8 October 1991. 1 specimen, AM P24058, $\mathrm{E}$ of Burwood Beach, $32^{\circ} 58^{\prime} \mathrm{S} 151^{\circ} 45^{\prime} \mathrm{E}, 28 \mathrm{~m}$, mud and gravel, shipek grab, 18 December 1975, HDWBS stn 06010304. 1 specimen, AM P58754, Cape Banks, $34^{\circ} 00^{\prime} \mathrm{S} 151^{\circ} 16^{\prime} \mathrm{E}, 45-50 \mathrm{~m}$, The Ecology Lab, January 1991. 1 specimen, P58755, SE of Bate Bay, $34^{\circ} 5.9^{\prime} \mathrm{S} 151^{\circ} 12^{\prime} \mathrm{E}$, 35-40 m, The Ecology Lab, January 1991, stn S2T4. 1 specimen, AM P58756, same locality, 35-40 m, The Ecology Lab, July 1990, stn S3T2. 3 specimens, AM P58757, same locality, 35-40 m, The Ecology Lab, January 1991, stn S3T4. 1 specimen, AM P46444, Bass Point, 34³6'S $150^{\circ} 54^{\prime} \mathrm{E}, 35-40 \mathrm{~m}$, The Ecology Lab, 1 February 1990, stn S1R3. 1 specimen, AM P58758, same locality, The Ecology Lab, November 1990, stn S1T3. 1 specimen, AM P52920, Wattamolla, $34^{\circ} 08^{\prime} \mathrm{S} 151^{\circ} 08.5^{\prime} \mathrm{E}$,

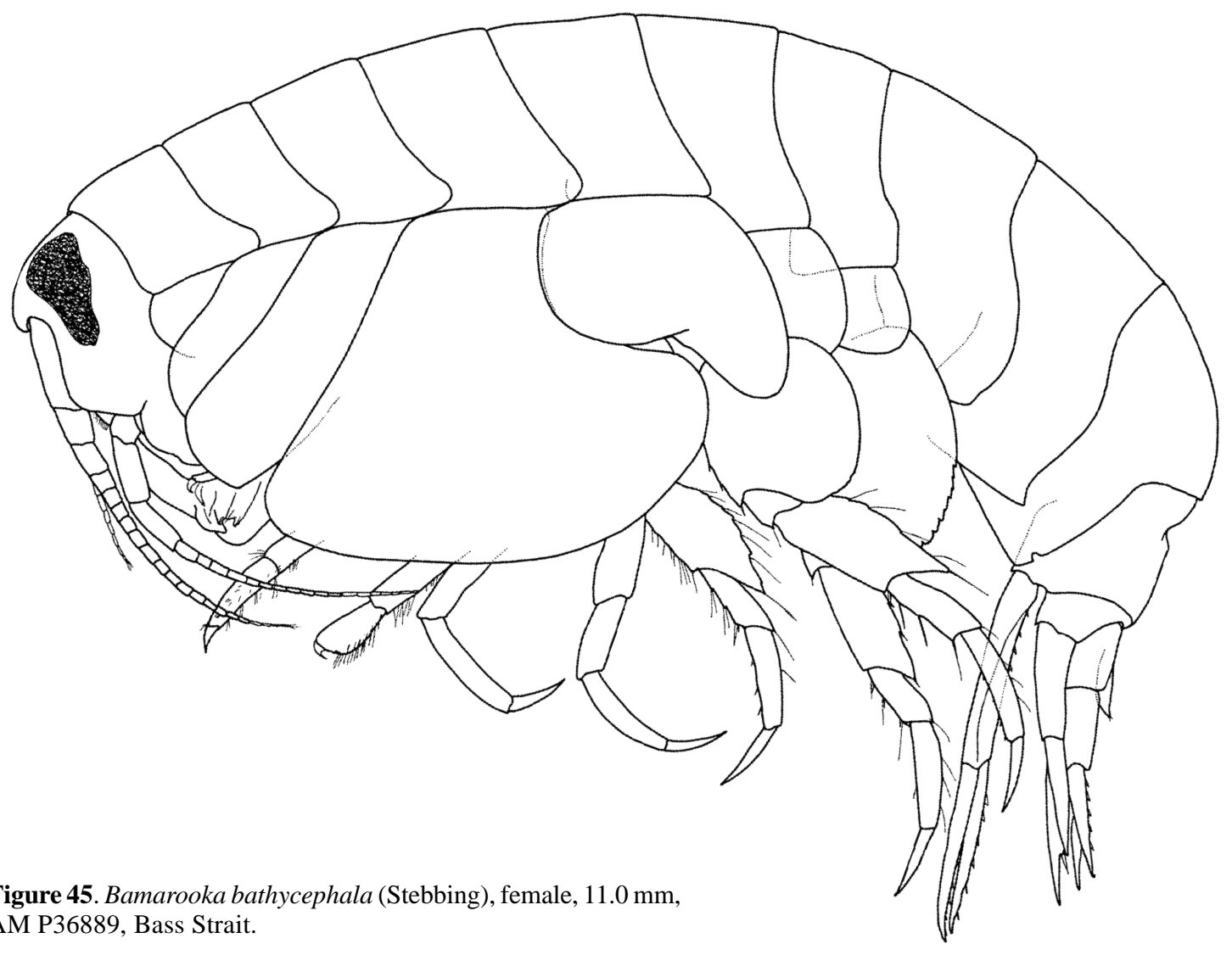

Figure 45. Bamarooka Strait.
AM P36889, Bass 


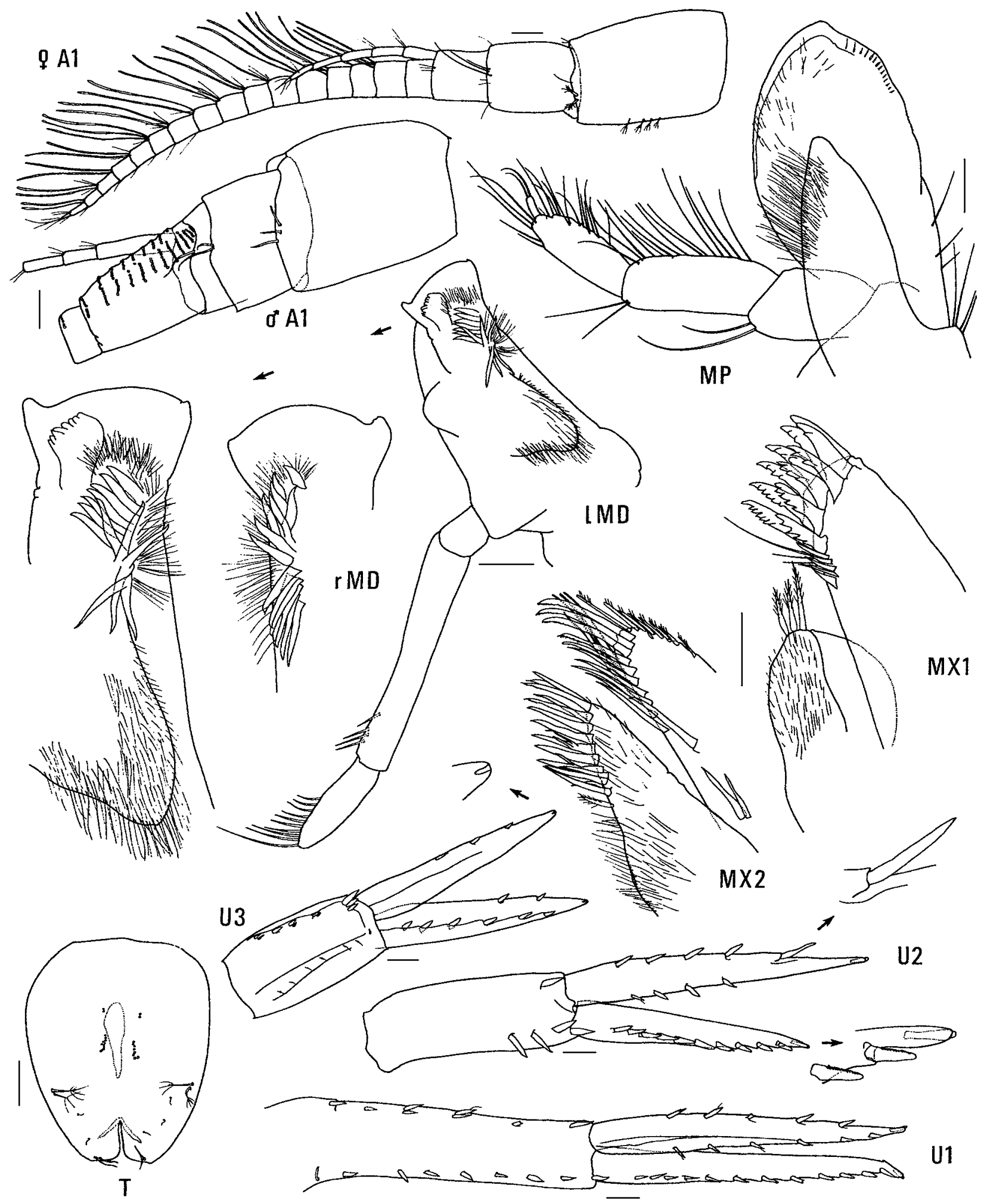

Figure 46. Bamarooka bathycephala (Stebbing), female, 11.0 mm, AM P36889; male, 7.2 mm, AM P36889; Bass Strait. Scales represent $0.1 \mathrm{~mm}$.

45-50 m, The Ecology Lab, June 1990, stn S2R4. BASS STRAIT: 9 specimens, NMV J2310, $42 \mathrm{~km} \mathrm{SW}$ of Babel Island, Tasmania, eastern Bass Strait, $40^{\circ} 13.8^{\prime} \mathrm{S} 148^{\circ} 39.6^{\prime} \mathrm{E}, 60 \mathrm{~m}$, muddy sand, epibenthic sled, R. Wilson, 14 November 1981, RV Tangaroa, stn BSS-165. 1 specimen, NMV J13928, $32 \mathrm{~km} \mathrm{SSW} \mathrm{of} \mathrm{Cape} \mathrm{Otway,} \mathrm{western} \mathrm{Bass} \mathrm{Strait,} \mathrm{39} 09^{\circ} \mathrm{S}$ $143^{\circ} 26^{\prime} \mathrm{E}, 85 \mathrm{~m}$, coarse carbonate sand, G.C.B. Poore, 8 October 1980 , HMAS Kimbla, cruise 80-K-1, stn BSS-55. 2 specimens, NMV J7450, $31 \mathrm{~km}$ SSW of Cape Otway, western Bass Strait, $39^{\circ} 08^{\prime} \mathrm{S} 143^{\circ} 24^{\prime} \mathrm{E}, 77$ m, medium sand, grab, G.C.B. Poore, 8 October 1980, HMAS Kimbla, stn BSS-56G. 1 specimen, NMV J7452, $35 \mathrm{~km} \mathrm{SSW}$ of Cape Otway, Victoria, western Bass Strait, $39^{\circ} 06^{\prime} \mathrm{S} 143^{\circ} 21^{\prime} \mathrm{E}, 59 \mathrm{~m}$, coarse sand, dredge, G.C.B. Poore, 8 October 1980, HMAS Kimbla, stn BSS-57D. 1 specimen, NMV J13929, 42 km NW of Cape Farewell, King Island, western Bass Strait, $39^{\circ} 19^{\prime} \mathrm{S} 143^{\circ} 38^{\prime} \mathrm{E}, 95 \mathrm{~m}$, coarse sand, carbonate, G.C.B. Poore, 10 October 1980, HMAS Kimbla, cruise 80-K-1, stn BSS 76. 10 specimens, NMV J13930, $6 \mathrm{~km}$ NE of Stanley, Tasmania, central Bass Strait, $40^{\circ} 48.8^{\prime} \mathrm{S} 145^{\circ} 22^{\prime} \mathrm{E}, 22 \mathrm{~m}$, fine sand, M. Gomon \& G.C.B. Poore, 4 November 1980, FRV Sarda, stn BSS 114. 9 specimens, NMV J2309, 25 


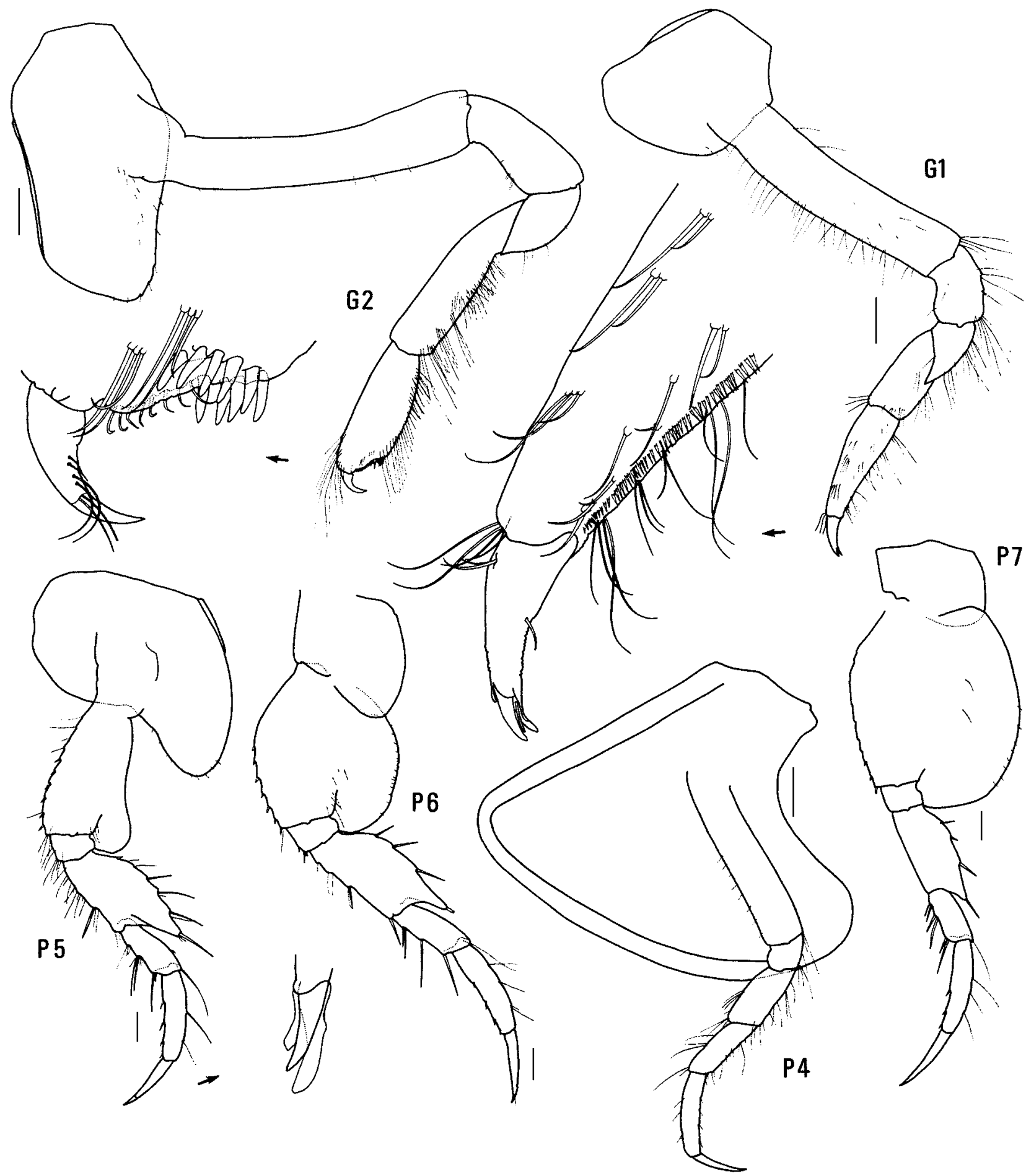

Figure 47. Bamarooka bathycephala (Stebbing), female, 11.0 mm, AM P36889, Bass Strait. Scales represent $0.2 \mathrm{~mm}$.

$\mathrm{km} \mathrm{S}$ of Cape Otway, Victoria, western Bass Strait, $39^{\circ} 06^{\prime} \mathrm{S} 143^{\circ} 35.8^{\prime} \mathrm{E}$, $95 \mathrm{~m}$, fine sand, dredge, M. Gomon et al., 31 January 1981, FRV Hai Kung, stn BSS-118D. 53 specimens, NMV J13931; 30 specimens, AM P36889; $26 \mathrm{~km} \mathrm{SW}$ of Cape Otway, Victoria, western Bass Strait, $39^{\circ} 01^{\prime} \mathrm{S}$ $143^{\circ} 22^{\prime} \mathrm{E}, 84 \mathrm{~m}$, medium sand, epibenthic sled, M. Gomon et al., 31 January 1981, FRV Hai Kung, stn BSS-120. 5 specimens, NMV J13932, $31 \mathrm{~km} \mathrm{~S}$ of Cape Otway, Victoria, western Bass Strait, $39^{\circ} 01.0^{\prime} \mathrm{S}$ $143^{\circ} 15.2^{\prime} \mathrm{E}, 84 \mathrm{~m}$, medium sand, epibenthic sled, M. Gomon et al., 31 January 1981, FRV Hai Kung, stn BSS-121. 2 specimens, NMV J48798, $94 \mathrm{~km} \mathrm{~N}$ of North Point, Flinders Island, eastern Bass Strait, 38 $53.7^{\circ} \mathrm{S}$ $147^{\circ} 55.2^{\prime} \mathrm{E}, 71 \mathrm{~m}$, medium sand, epibenthic sled, R. Wilson, 17 November 1981, RV Tangaroa, stn BSS 171S. 8 specimens, NMV J3769, $25 \mathrm{~km} \mathrm{~S}$ of Aireys Inlet, Victoria, central Bass Strait, 38 $44.6^{\prime} \mathrm{S} 144^{\circ} 09.0^{\prime} \mathrm{E}, 77 \mathrm{~m}$, fine sand, epibenthic sled, R. Wilson, 19 November 1981, RV Tangaroa, stn BSS-182. 1 specimen, NMV J3772, 15 km SW of Point Reginald, Victoria, western Bass Strait, $38^{\circ} 50.0^{\prime} \mathrm{S} 143^{\circ} 07.5^{\prime} \mathrm{E}, 69 \mathrm{~m}$, fine sand, grab, R. Wilson, 20 November 1981, RV Tangaroa, stn BSS-186. 2 specimens, NMV J13933, $70 \mathrm{~km} \mathrm{SW}$ of Cape Otway, Victoria, western Bass Strait, $39^{\circ} 26.3^{\prime} \mathrm{S} 143^{\circ} 06.8^{\prime} \mathrm{E}, 115 \mathrm{~m}$, sand, epibenthic sled, R. Wilson, 21 November 1981, RV Tangaroa, stn BSS-194. 6 specimens, NMV J13934, $65 \mathrm{~km} \mathrm{~S}$ of Cape Schanck, Victoria, central Bass Strait, 39 08.3'S $144^{\circ} 43.9^{\prime} \mathrm{E}, 66 \mathrm{~m}$, coarse sand, R. Wilson, 23 November 1981, RV Tangaroa, stn BSS-201.

Type locality. Off mouth of Port Phillip Bay, Bass Strait, Australia, $38^{\circ} 22.5^{\prime} \mathrm{S} 144^{\circ} 36.5^{\prime} \mathrm{E}, 60 \mathrm{~m}$ depth. 
Description. Based on female, $11 \mathrm{~mm}$, AM P36889. Head much deeper than long, anterior margin with notch extended into a slit; rostrum present, anteriorly rounded; eye present, ventrally tapered. Antenna 1 peduncular article 1 not ballshaped proximally, distal margin with small medial spine; peduncular article 2 medium length; flagellum without callynophore, calceoli absent. Antenna 2 flagellum about as long as that of antenna 1, without calceoli. Mouthpart bundle subconical. Epistome/upper lip with broad midanterior bulge (lateral view). Mandible lacinia mobilis a stemmed, distally-cusped blade; accessory setal row with intermediate setae; palp article 2 with 4 posterodistal setae, article 3 without A3-seta. Maxilliped outer plate with distal margin smooth, medial margin without notch.

Gnathopod 1 carpus shorter than propodus $(0.8 \times)$; propodus, posterior margin without robust setae. Gnathopod 2 palm acute, with 3 lateral robust setae, 6 medial robust setae. Pereopods 3 and 4 merus and carpus without setal fringe. Pereopod 4 coxa with anterior margin slightly obtuse, posterior margin rounded, anteroventral corner rounded. Pereopods 5-7 with distal articles elongate, dactyli long and slender. Pereopod 5 basis expanded posterodistally (pear-shaped). Pereopod 7 basis subrectangular, posteroventral corner rounded, posteroventral margin straight.

Epimeron 3 posterior margin smooth, with notch well above rounded posteroventral corner. Uropod 1 peduncle dorsolateral margin with 11 robust setae; outer ramus without large spines between robust setae. Uropod 2 inner ramus slightly constricted. Uropod 3 rami lanceolate; without plumose setae; outer ramus 1-articulate. Telson slightly cleft (about 20\%).

Male (sexually dimorphic characters). Based on male, 7.2 $\mathrm{mm}$, AM P36889. Antenna 1 flagellum with callynophore. Antenna 2 flagellum with calceoli. Gnathopod 2 palm without lateral robust setae, with 2 medial robust setae.

Variation. This species shows considerable variation in the robust-setae formula of gnathopod 2 palm. This variation is correlated with the size and/or sex of specimens. For males the range is from $0 / 1$ through $0 / 2$ to $1 / 2$; for females the known range is from $1 / 4$ through $2 / 4,4 / 4$ to $4 / 5$. Stebbing (1888: 701) recorded "a row of three short stout spines [= robust setae]... on one side and one or two more on the other".

An $8.3 \mathrm{~mm}$ ovigerous female from east of Burwood Beach, NSW, differs slightly from the Bass Strait population in that the rostrum is slightly less produced, the eye is not quite so tapered and the basis of pereopod 5 is slightly broader distally.

Remarks. Most species of Bamarooka (B. bathycephala, B. dinjerra, B. endota and B. kimbla) have ventrally tapered eyes. Bamarooka bathycephala differs from all of these species in the basis of pereopod 5, which is pear-shaped.

Habitat. Bamarooka bathycephala has been collected frequently on sandy sediments and occasionally on mud.

Distribution. Southeastern Australia, from Sydney to Bass Strait; 22-115 m depth.

\section{Bamarooka dinjerra n.sp.}

Figs. $48-50$

Type material. Holotype, female, $4.8 \mathrm{~mm}$, with 4 embryos, AM P36890; 1 PARATYPE, male, $3.2 \mathrm{~mm}$, AM P36891; northwest of Port Hedland, North West Shelf, Western Australia, eastern Indian Ocean, $20^{\circ} 00.3^{\prime} \mathrm{S}$ $117^{\circ} 00.4^{\prime} \mathrm{E}$ to $20^{\circ} 00.5^{\prime} \mathrm{S} 117^{\circ} 00.0^{\prime} \mathrm{E}, 53 \mathrm{~m}$, epibenthic sled, 22 February 1983, FRV Soela, CSIRO NWS stn 01.B17.S. 1 PARATYPE, AM P58123, same locality, $19^{\circ} 05.2^{\prime} \mathrm{S}$ $118^{\circ} 57.6^{\prime} \mathrm{E}$ to $19^{\circ} 05.3^{\prime} \mathrm{S} 118^{\circ} 56.7^{\prime} \mathrm{E}, 82 \mathrm{~m}$, epibenthic sled, 14 February 1983, FRV Soela, CSIRO NWS stn 01.B10.S. 5 PARATYPES, AM P36892, same locality, $19^{\circ} 59.3^{\prime} \mathrm{S}$ $117^{\circ} 03.6^{\prime} \mathrm{E}$ to $19^{\circ} 59.6^{\prime} \mathrm{S} 117^{\circ} 03.0^{\prime} \mathrm{E}, 52 \mathrm{~m}$, epibenthic sled, 22 February 1983, FRV Soela, CSIRO NWS stn 01.B16.S.

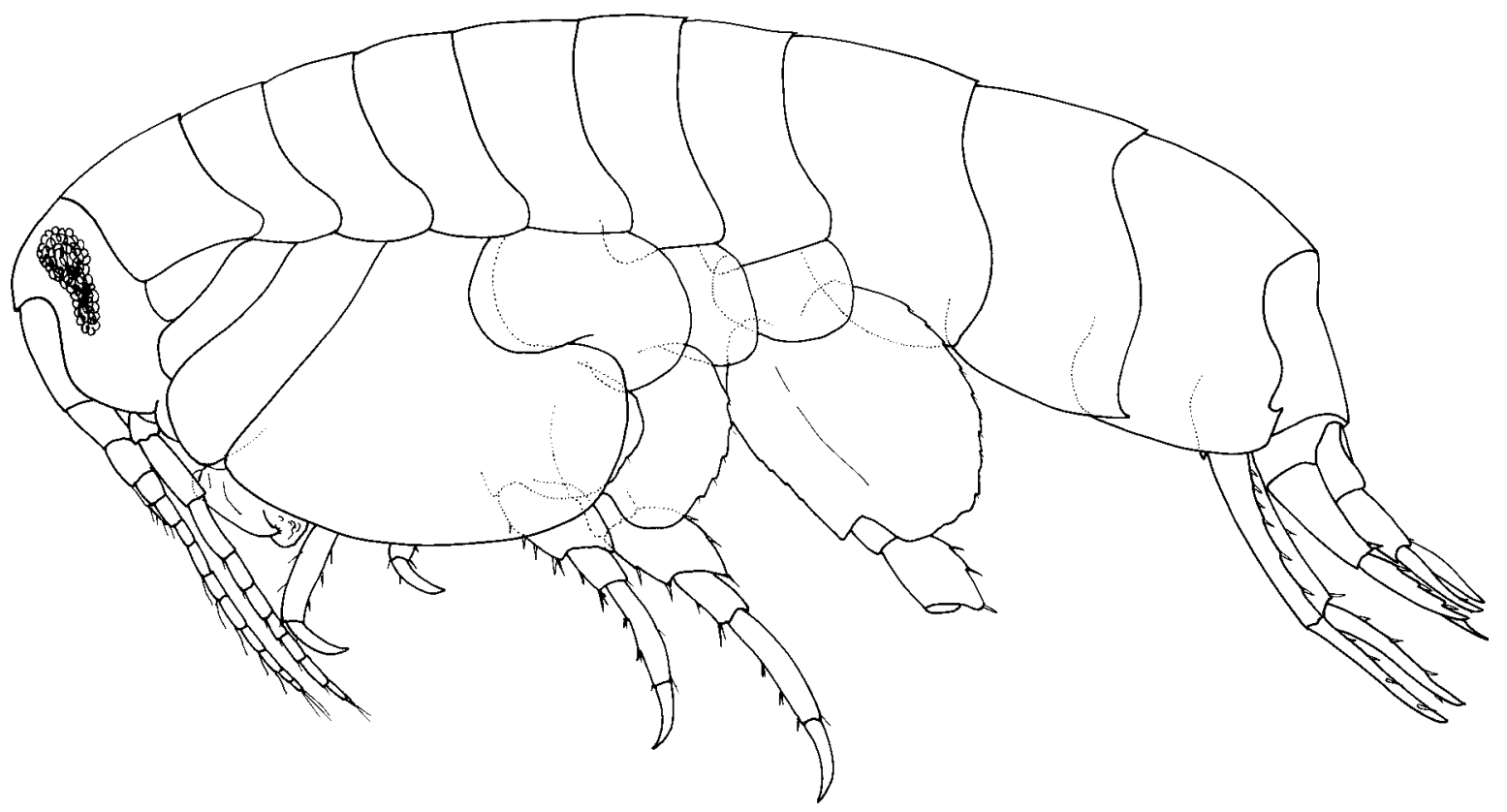

Figure 48. Bamarooka dinjerra n.sp., paratype female, 3.5 mm, AM P36892; North West Shelf, Western Australia. 


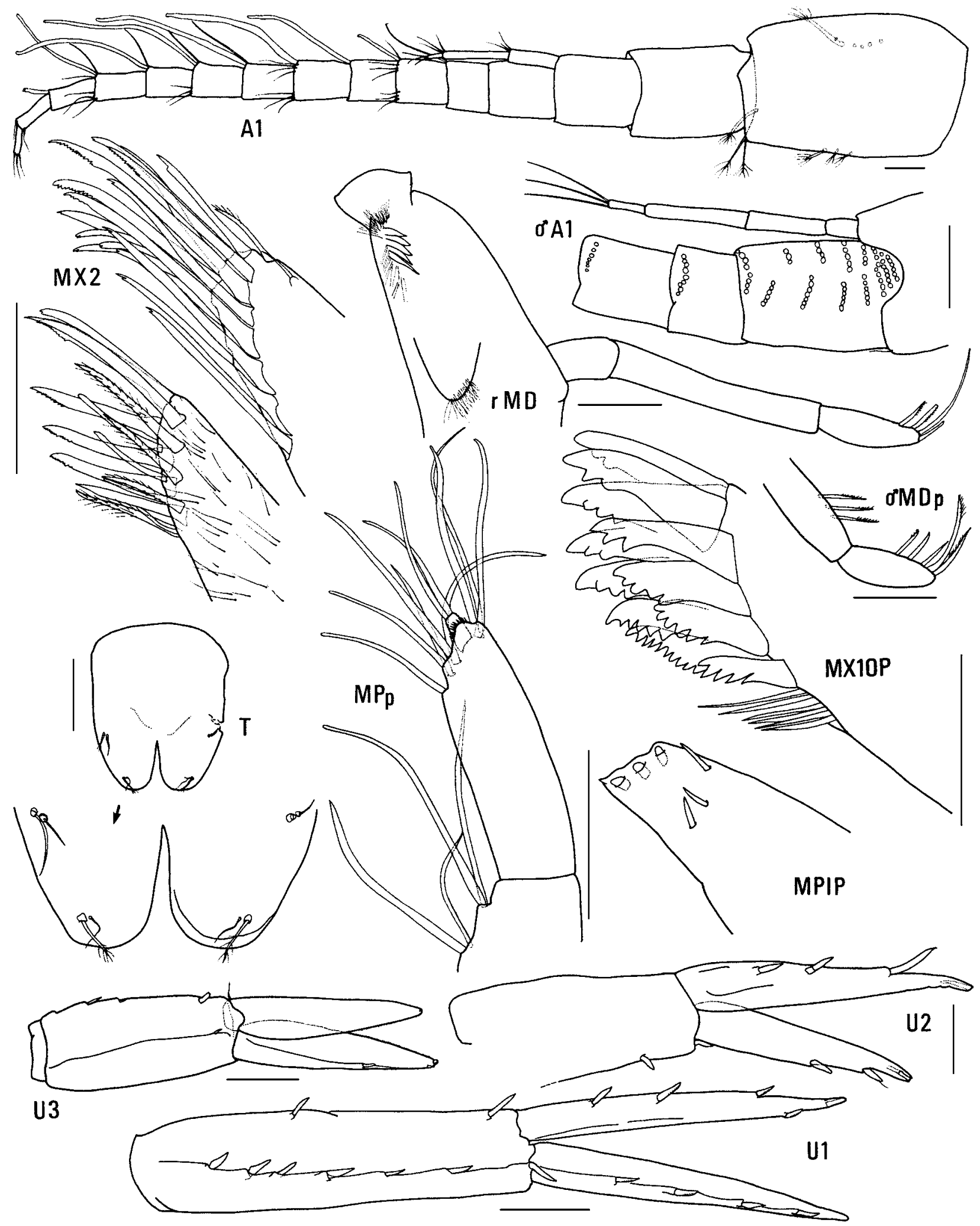

Figure 49. Bamarooka dinjerra n.sp., holotype female, 4.8 mm, AM P36890; paratype male, 3.2 mm, AM P36891, North West Shelf, Western Australia. Scales for U1-3, T represent $0.1 \mathrm{~mm}$; remainder represent $0.05 \mathrm{~mm}$.

1 PARATYPE, AM P36893, same locality, 1956.9'S $117^{\circ} 53.7^{\prime} \mathrm{E}$ to $19^{\circ} 56.4^{\prime} \mathrm{S} 117^{\circ} 53.8^{\prime} \mathrm{E}, 42-43 \mathrm{~m}$, epibenthic sled, 22 April 1983, FRV Soela, CSIRO NWS stn 02.B2.S. 1 PARATYPE, AM P36894, same locality, $19^{\circ} 28.6^{\prime}$ S $118^{\circ} 55.0^{\prime} \mathrm{E}$ to $19^{\circ} 28.2^{\prime} \mathrm{S} 118^{\circ} 55.5^{\prime} \mathrm{E}, 40-38 \mathrm{~m}$, epibenthic sled, 26 April 1983, FRV Soela, CSIRO NWS stn 02.B9.S. 1 PARATYPE, AM P36895, same locality, $20^{\circ} 00.2^{\prime} \mathrm{S} 117^{\circ} 00.5^{\prime} \mathrm{E}$ to $20^{\circ} 00.4^{\prime} \mathrm{S} 116^{\circ} 59.9^{\prime} \mathrm{E}, 52 \mathrm{~m}$, epibenthic sled, 4 September 1983, FRV Soela, CSIRO NWS stn 04.B17.S. 1 PARATYPE, AM P36896, same locality, $19^{\circ} 55.5^{\prime} \mathrm{S} 117^{\circ} 55.8^{\prime} \mathrm{E}$ to $19^{\circ} 55.1^{\prime} \mathrm{S}$ 


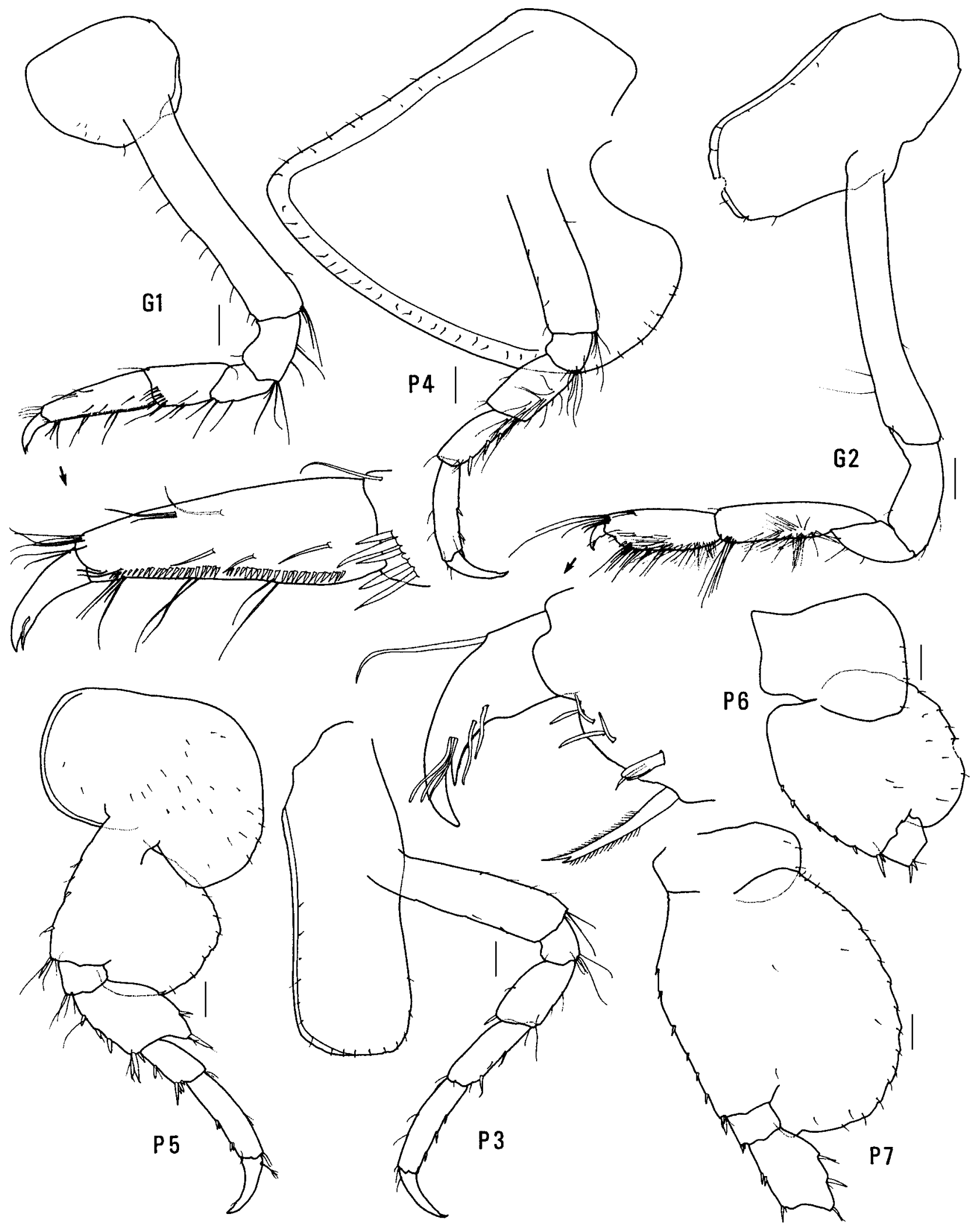

Figure 50. Bamarooka dinjerra n.sp., holotype female, 4.8 mm, AM P36890; North West Shelf, Western Australia. Scales represent $0.1 \mathrm{~mm}$.

$117^{\circ} 56.2^{\prime} \mathrm{E}, 40-41 \mathrm{~m}$, epibenthic sled, 26 October 1983, FRV Soela, CSIRO NWS stn 05.B3.S. 1 PARATYPE, NMV J13936, same locality, $20^{\circ} 01^{\prime} \mathrm{S} 117^{\circ} 17^{\prime} \mathrm{E}$ to $20^{\circ} 00^{\prime} \mathrm{S} 117^{\circ} 18^{\prime} \mathrm{E}, 46 \mathrm{~m}$, coarse shell, dredge, G.C.B. Poore \& H.M. Lew Ton, FRV Soela, 2 June 1983, FRV Soela, NMV stn NWA-5.
Type locality. Northwest of Port Hedland, North West Shelf, Western Australia, eastern Indian Ocean, $20^{\circ} 00.3^{\prime} \mathrm{S}$ $117^{\circ} 00.4^{\prime} \mathrm{E}$ to $20^{\circ} 00.5^{\prime} \mathrm{S} 117^{\circ} 00.0^{\prime} \mathrm{E}, 53 \mathrm{~m}$ depth. 
Description. Based on holotype female, $4.8 \mathrm{~mm}$, (AM P36890). Head much deeper than long, anterior margin with notch extended into a slit; rostrum present, anteriorly rounded; eye present, ventrally tapered. Antenna 1 peduncular article 1 not ball-shaped proximally, distal margin with small medial spine; peduncular article 2 medium length; flagellum without callynophore, calceoli absent. Antenna 2 flagellum about as long as that of antenna 1, without calceoli. Mouthpart bundle subconical. Epistome/ upper lip almost straight (lateral view). Mandible lacinia mobilis a stemmed, distally-cusped blade; accessory setal row with intermediate setae; palp article 2 without posterodistal setae, article 3 without A3-seta. Maxilliped outer plate with distal margin smooth, medial margin without notch.

Gnathopod 1 carpus subequal in length to propodus $(0.9 \times)$; propodus, posterior margin without robust setae. Gnathopod 2 palm acute, with no lateral robust setae, 1 medial robust seta. Pereopods 3 and 4 merus and carpus without setal fringe. Pereopod 4 coxa with anterior margin slightly obtuse, posterior margin rounded, anteroventral corner rounded. Pereopods 5-7 with distal articles elongate, dactyli long and slender. Pereopod 5 basis expanded posteriorly, rounded with sloping posteroproximal shoulder. Pereopod 7 basis rounded posteriorly, posteroventral corner rounded, posteroventral margin curved.

Epimeron 3 posterior margin smooth, with notch well above rounded posteroventral corner. Uropod 1 peduncle dorsolateral margin with 7 robust setae; outer ramus without large spines between robust setae. Uropod 2 inner ramus slightly constricted. Uropod 3 rami lanceolate; without plumose setae; outer ramus 1-articulate. Telson moderately cleft (about 33\%).

Male (sexually dimorphic characters). Based on paratype male, $3.2 \mathrm{~mm}$, AM P36891. Antenna 1 flagellum with callynophore. Antenna 2 flagellum with calceoli. Mandible palp article 2 with 3 posterodistal setae.
Etymology. The specific name is an Australian Aboriginal word meaning "west".

Remarks. Most species of Bamarooka (B. bathycephala, $B$. dinjerra, B. endota and B. kimbla) have ventrally tapered eyes. Bamarooka dinjerra and $B$. endota are very similar. They differ in the basis of pereopod 5, which has a sloped proximal posterior shoulder in $B$. dinjerra and is almost round in B. endota.

Habitat. Generally not known; taken on one occasion on a coarse shell bottom.

Distribution. North West Shelf, off Western Australia, Indian Ocean; 40-82 m depth.

\section{Bamarooka endota n.sp.}

Figs. 51-54

Amaryllis macrophthalma.-Stebbing, 1910a: 569 (in part, part = A. kamata).

Type material. HOLOTYPE, female, $7.5 \mathrm{~mm}$, ovigerous (5 eggs), NMV J13937; 1 PARATYPE, male, 5.9 mm, NMV J13938; 10 PARATYPES, $4.0-8.8 \mathrm{~mm}$, AM P36897; $66 \mathrm{~km}$ south of Rodondo Island, central Bass Strait, Australia, 39 $48.6^{\prime} \mathrm{S}$ $146^{\circ} 18.8^{\prime} \mathrm{E}, 82 \mathrm{~m}$, sand, silt and mud, epibenthic sled, R. Wilson, 23 November 1981, RV Tangaroa, cruise 81-T-1, stn BSS-158S. 10 PARATYPES, AM P59022; 23 PARATYPES, NMV J13939; $57 \mathrm{~km} \mathrm{~S}$ of Rodondo Island, central Bass Strait, Australia, $39^{\circ} 46.0^{\prime} \mathrm{S} 146^{\circ} 18.0^{\prime} \mathrm{E}, 80 \mathrm{~m}$, muddy shell, epibenthic sled, R. Wilson, 13 November 1981, RV Tangaroa, stn BSS159S. 4 PARATYPES, NMV J13945, $57 \mathrm{~km}$ S of Rodondo Island, central Bass Strait, Australia, $39^{\circ} 43.5^{\prime} \mathrm{S} 146^{\circ} 18.8^{\prime} \mathrm{E}, 80 \mathrm{~m}$, muddy shell, grab, G.C.B. Poore, 13 November 1981, RV Tangaroa, stn BSS-159G.

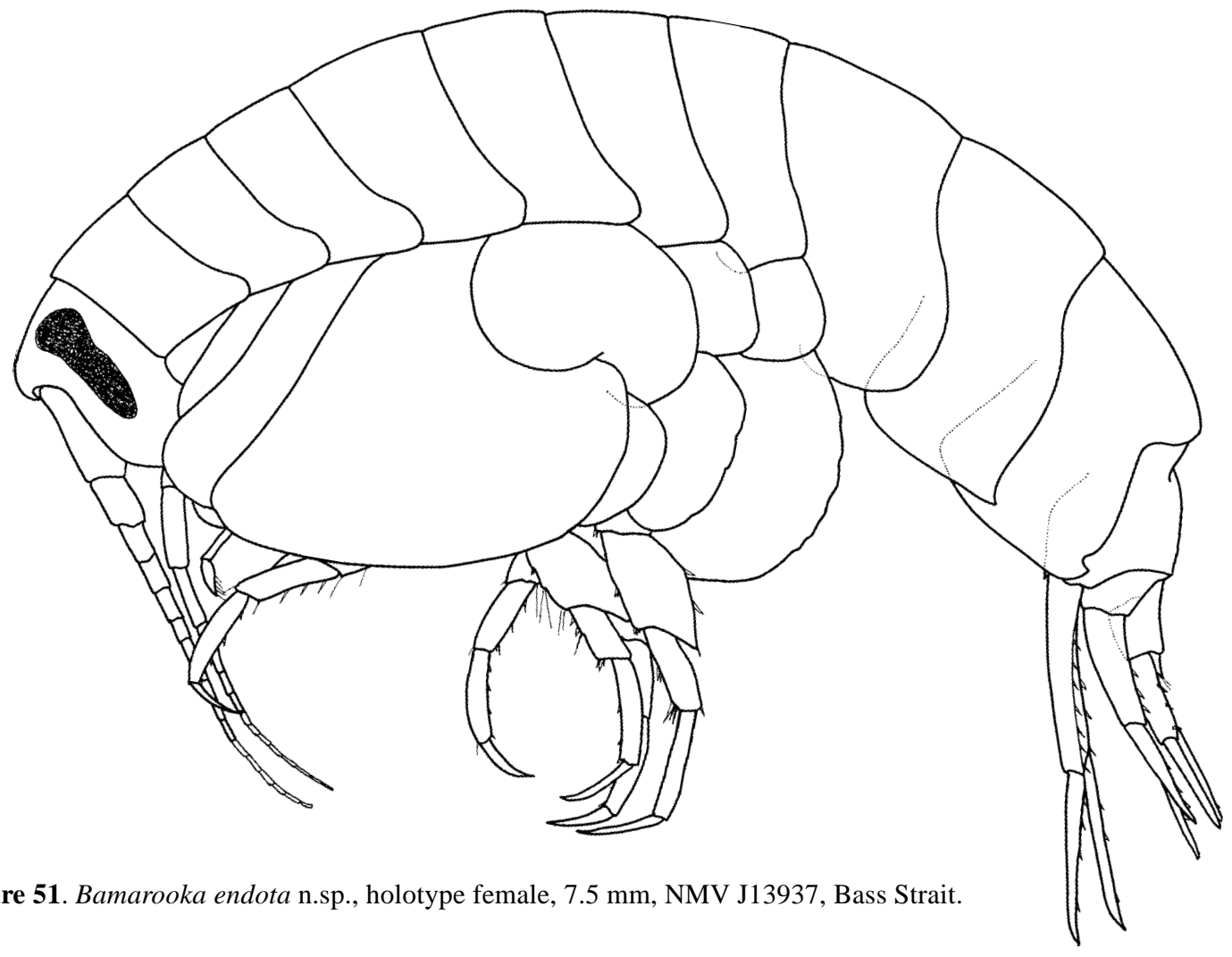




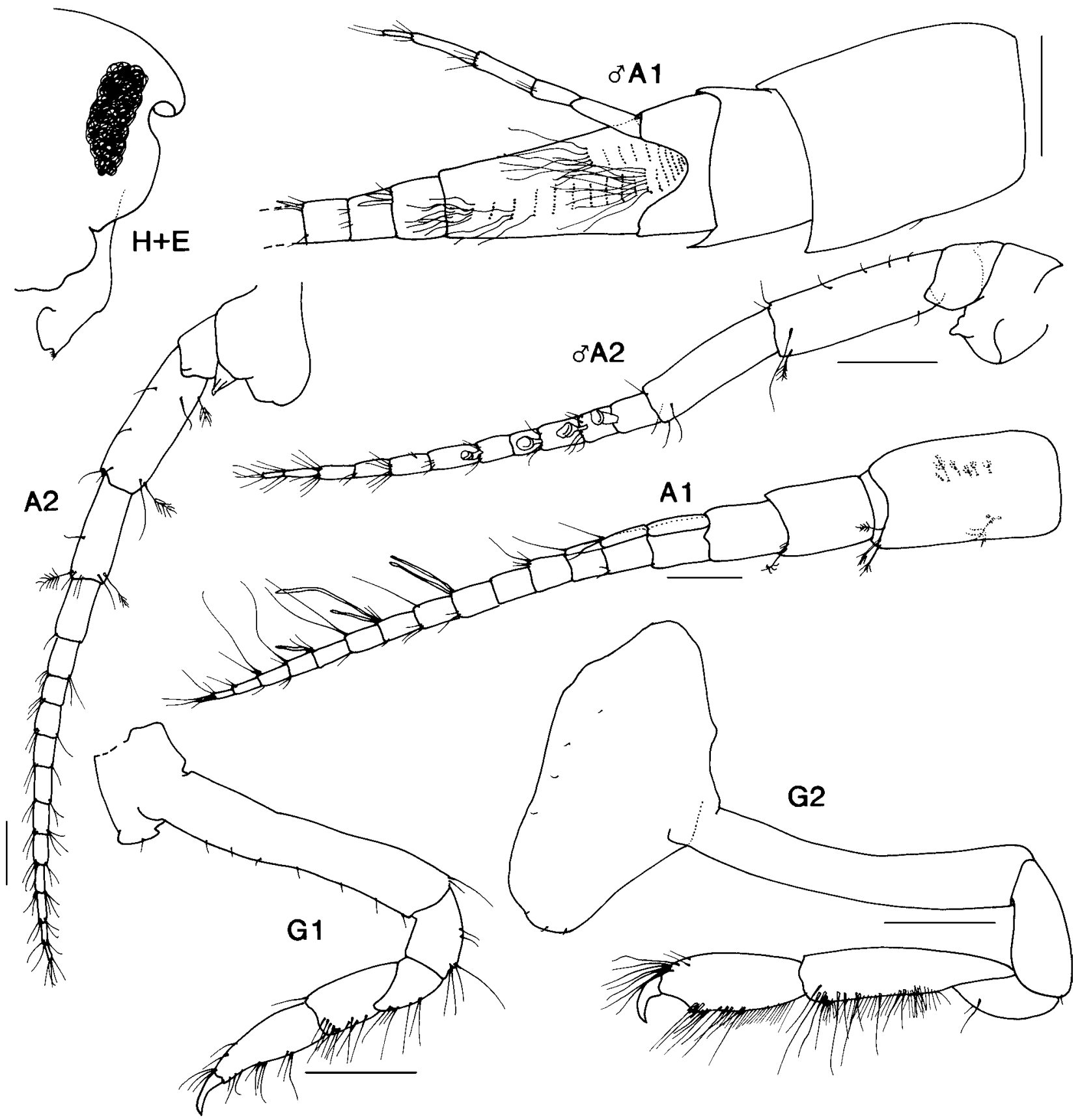

Figure 52. Bamarooka endota n.sp., holotype female, 7.5 mm, NMV J13937; paratype male, 5.9 mm, NMV J13938; Bass Strait. Scales for A1, 2 represent $0.2 \mathrm{~mm}$; remainder represent $0.5 \mathrm{~mm}$.

Additional material. New SOUTH WALES: 1 specimen, AM P36904, E of Newcastle, $32^{\circ} 53^{\prime} \mathrm{S} 152^{\circ} 35^{\prime} \mathrm{E}, 146-165 \mathrm{~m}, 15$ August 1985, FRV Kapala, stn K85-12-23. 12 specimens, AM P37397, SE of Broken Bay, $33^{\circ} 36^{\prime} \mathrm{S} 151^{\circ} 30^{\prime} \mathrm{E}$ to $33^{\circ} 37^{\prime} \mathrm{S} 151^{\circ} 29^{\prime} \mathrm{E}, 71-75 \mathrm{~m}$, benthic trawl, 10 February 1986, Kapala, stn K86-01-02. 2 females, 2 males, AM P36905, E of Long Reef Point, $33^{\circ} 43^{\prime} \mathrm{S} 151^{\circ} 46^{\prime} \mathrm{E}$ to $33^{\circ} 44^{\prime} \mathrm{S} 151^{\circ} 46^{\prime} \mathrm{E}, 174 \mathrm{~m}$, trawl, 19 December 1985, FRV Kapala, stn K85-21-08. 5 specimens,

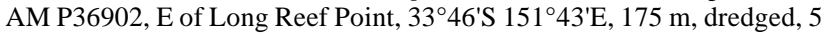
December 1977, FRV Kapala, stn K77-23-01. 5 females, AM P36903, E of Bondi, $33^{\circ} 52^{\prime} \mathrm{S} 151^{\circ} 23^{\prime} \mathrm{E}, 80 \mathrm{~m}$, dredged, 11 December 1980, FRV Kapala, stn K80-20-11. 1 male, AM P22060, E of Malabar, 3358.0'S $151^{\circ} 20.6^{\prime} \mathrm{E}, 75 \mathrm{~m}$, sandy gravel, shipek grab, 25 May 1972, AMSBS stn C6. 2 females, AM P36899, 8-9.5 km E of Coogee, [approx. 33 57 'S $\left.151^{\circ} 21.5^{\prime} \mathrm{E}\right], 90-91 \mathrm{~m}$, fine sand, E.R. Waite, 15 March 1898, HMCS Thetis, stn 44. 1 specimen, AM P36901, 4-6 km E of Botany Bay, [approx. $\left.34^{\circ} 01^{\prime} \mathrm{S} 151^{\circ} 19^{\prime} \mathrm{E}\right], 60-102 \mathrm{~m}$, trawl, F.A. McNeill \& A. Livingstone, August 1921, ST Goonambee. 1 specimen, AM P37398, E of Port Hacking, [approx. $34^{\circ} 03^{\prime} \mathrm{S} 151^{\circ} 12^{\prime} \mathrm{E}$ ], 40-69 m, sand, E.R. Waite, 10 March 1898, HMCS Thetis, stn 35. 1 female, AM P36898, 3-4 km off
Botany Bay, [approx. $34^{\circ} 05^{\prime} \mathrm{S} 151^{\circ} 15^{\prime} \mathrm{E}$ ], 91-95 m, mud, E.R. Waite, 11 March 1898, HMCS Thetis, stn 37. 3 females, AM P36900, 5.5-6.5 km E of Wattamolla, [approx. $34^{\circ} 10^{\prime} \mathrm{S} 151^{\circ} 11^{\prime} \mathrm{E}$ ], 108-99 m, mud, E.R. Waite, 22 March 1898, HMCS Thetis, stn 57. 2 specimens, AM P36908, off Montague Island, [approx. $36^{\circ} 15^{\prime} \mathrm{S} 150^{\circ} 14^{\prime} \mathrm{E}$ ], from stomach of jackass fish Nemadactylus macropterus, April 1939, K. Sheard Collection. BASS STRAIT: 2 specimens, AM P58119, eastern Bass Strait, $38^{\circ} 59.1^{\prime} \mathrm{S}$ $148^{\circ} 31.6^{\prime} \mathrm{E}, 125 \mathrm{~m}$, benthic sled, P.B. Berents, 27 August 1994, FRV Southern Surveyor, stn SS 05/94/60. 1 male, 3 females, NMV J13940, $85 \mathrm{~km}$ NE of North Point, Flinders Island, eastern Bass Strait, $39^{\circ} 02.4^{\prime} \mathrm{S}$ $148^{\circ} 30.6^{\prime} \mathrm{E}, 120 \mathrm{~m}$, muddy sand, R. Wilson, 15 November 1981 , RV Tangaroa, stn BSS-169. 5 specimens, NMV J13941, $100 \mathrm{~km} \mathrm{NE}$ of North Point, Flinders Island, eastern Bass Strait, $31^{\circ} 51.8^{\prime} \mathrm{S} 148^{\circ} 26.5^{\prime} \mathrm{E}, 130 \mathrm{~m}$, fine sand, epibenthic sled, R. Wilson, 15 November 1981, RV Tangaroa, stn BSS-170S. 1 female, NMV J7628, 100 km NE of North Point, Flinders Island, eastern Bass Strait, $31^{\circ} 51.8^{\prime} \mathrm{S} 148^{\circ} 26.5^{\prime} \mathrm{E}, 130 \mathrm{~m}$, fine sand, grab, R. Wilson, 15 November 1981, RV Tangaroa, stn BSS-170G. 18 specimens, NMV J13942, $38 \mathrm{~km}$ SW of Cape Paterson, central Bass Strait, $38^{\circ} 56.4^{\prime} \mathrm{S} 145^{\circ} 16.6^{\prime} \mathrm{E}, 70 \mathrm{~m}$, fine sand, epibenthic sled, R.Wilson, 12 


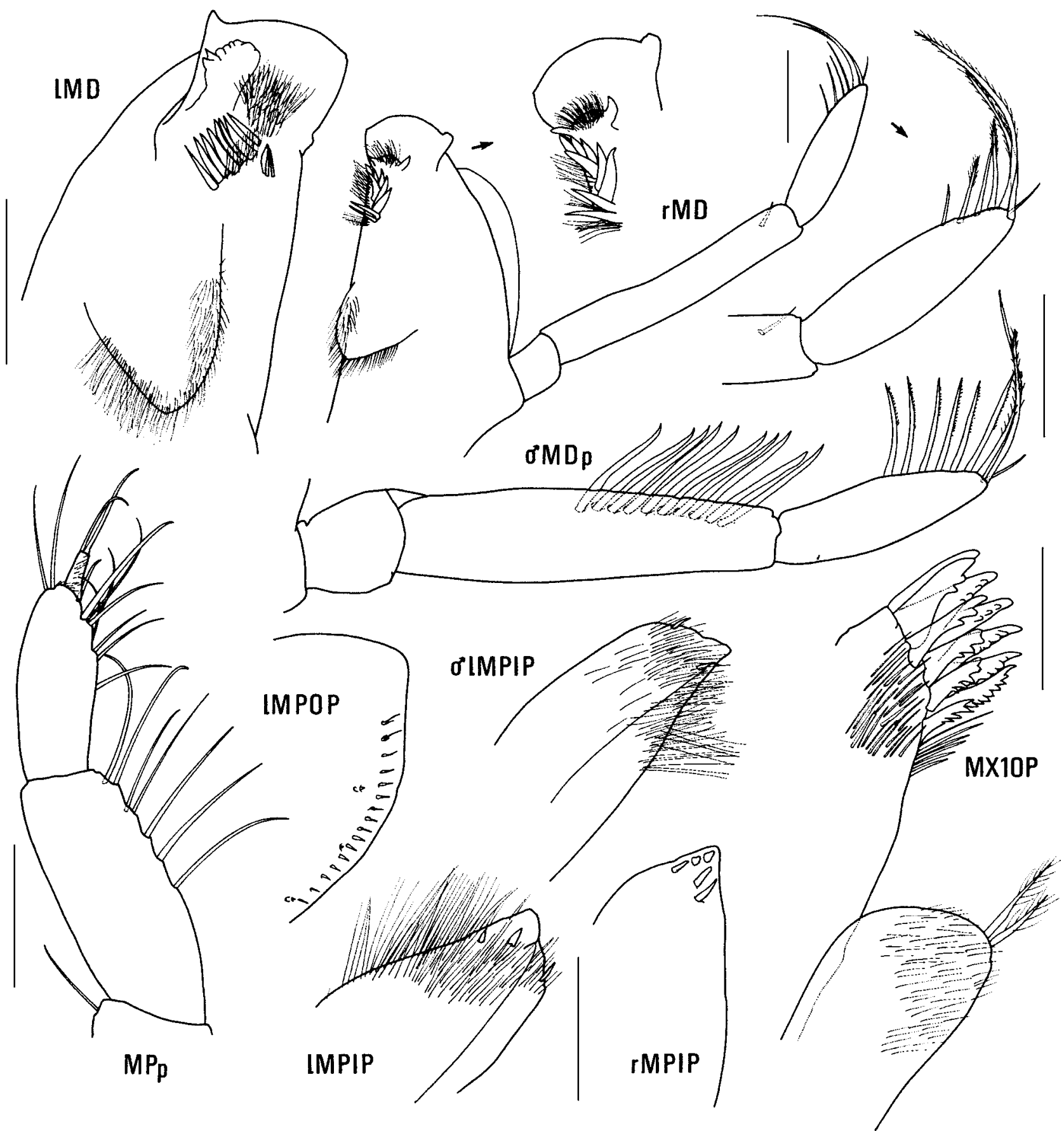

Figure 53. Bamarooka endota n.sp., holotype female, 7.5 mm, NMV J13937; paratype male, 5.9 mm, NMV J13938; Bass Strait. Scales represent $0.1 \mathrm{~mm}$

November 1981, RV Tangaroa, stn BSS-155S. 8 specimens, NMV J13943, $100 \mathrm{~km}$ SSE of Cape Liptrap, central Bass Strait, Australia, $39^{\circ} 45.9^{\prime} \mathrm{S} 145^{\circ} 33.5^{\prime} \mathrm{E}, 74 \mathrm{~m}$, muddy fine sand, epibenthic sled, R. Wilson, 13 November 1981, RV Tangaroa, stn BSS-156S. 1 specimen NMV J13944, 65 km ENE of Cape Rochon, Three Hummock Island, 40 $10.9^{\prime} \mathrm{S}$ $145^{\circ} 44.3^{\prime} \mathrm{E}, 75 \mathrm{~m}$, shell, bryozoans and mud, epibenthic sled, R. Wilson, 13 November 1981, RV Tangaroa, stn BSS-157S.

Type locality. $66 \mathrm{~km}$ south of Rodondo Island, central Bass Strait, Australia, $39^{\circ} 48.6^{\prime} \mathrm{S} 146^{\circ} 18.8^{\prime} \mathrm{E}, 82 \mathrm{~m}$ depth.

Description. Based on holotype female, $7.5 \mathrm{~mm}$, NMV J13937. Head much deeper than long, anterior margin with notch extended into a slit; rostrum present, anteriorly truncated; eye present, ventrally tapered. Antenna 1 peduncular article 1 not ball-shaped proximally, distal margin without a medial spine; peduncular article 2 medium length; flagellum without callynophore, calceoli absent. Antenna 2 flagellum about as long as that of antenna 1, without calceoli. Mouthpart bundle subconical. Epistome/ upper lip with broad mid-anterior bulge (lateral view). Mandible lacinia mobilis a stemmed, distally-cusped blade; accessory setal row with intermediate setae; palp article 2 with 1 posterodistal seta, article 3 without A3-seta. Maxilliped outer plate with distal margin smooth, medial margin without notch.

Gnathopod 1 carpus subequal in length to propodus $(0.9 \times)$; propodus, posterior margin without robust setae. Gnathopod 2 palm slightly acute, with no lateral robust setae, 3 medial robust setae. Pereopods 3 and 4 merus and 


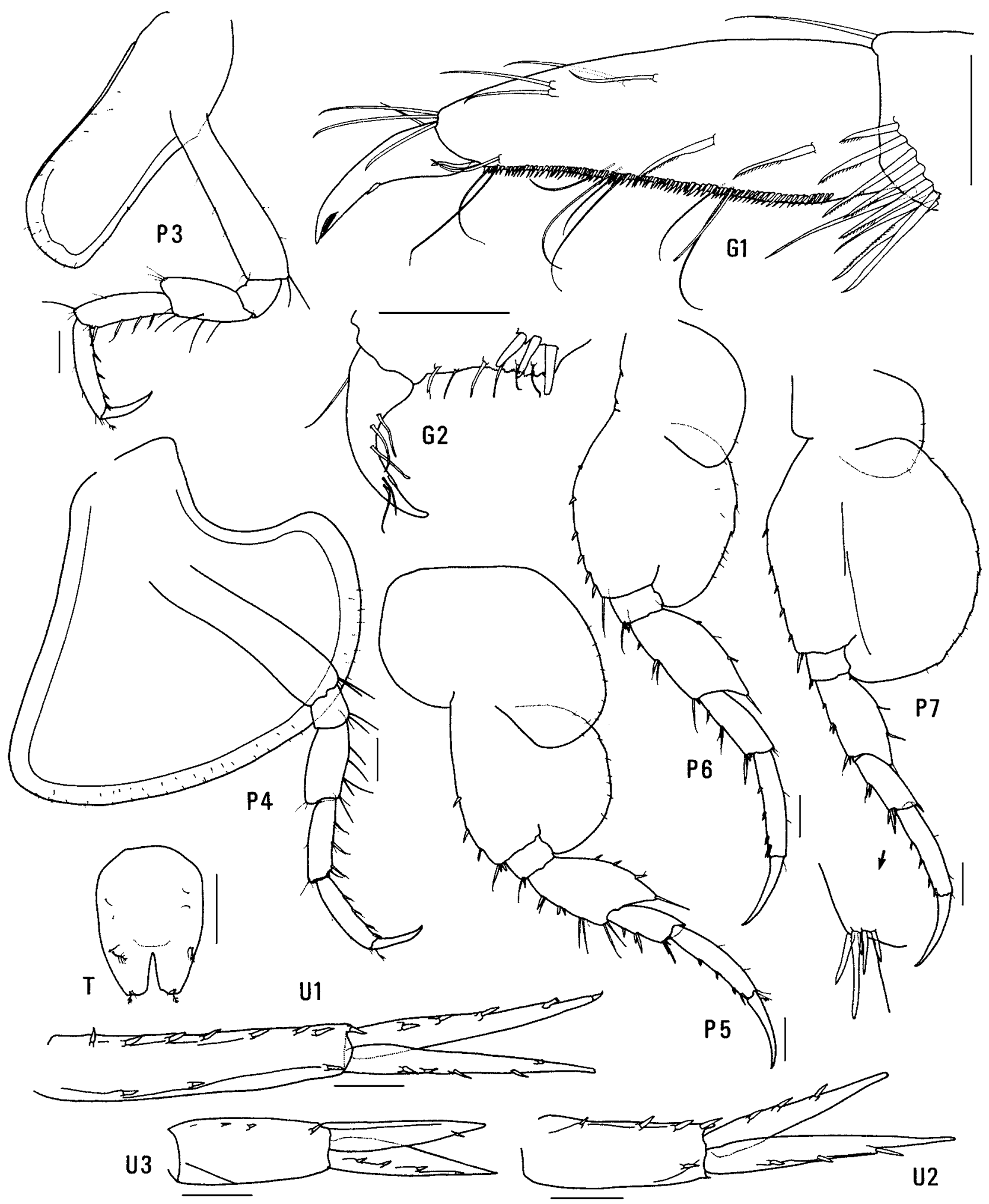

Figure 54. Bamarooka endota n.sp., holotype female, 7.5 mm, NMV J13937, Bass Strait. Scales for G1, 2 represent $0.1 \mathrm{~mm}$; remainder represent $0.2 \mathrm{~mm}$.

carpus without setal fringe. Pereopod 4 coxa with anterior margin slightly obtuse, posterior margin rounded, anteroventral corner rounded. Pereopods 5-7 with distal articles elongate, dactyli long and slender. Pereopod 5 basis expanded posteriorly, rounded. Pereopod 7 basis rounded posteriorly, posteroventral corner rounded, posteroventral margin curved.
Epimeron 3 posterior margin smooth, with notch well above rounded posteroventral corner. Uropod 1 peduncle dorsolateral margin with 8 robust setae; outer ramus without large spines between robust setae. Uropod 2 inner ramus slightly constricted. Uropod 3 rami lanceolate; without plumose setae; outer ramus 1-articulate. Telson slightly cleft (about 28\%). 
Male (sexually dimorphic characters). Based on paratype male, $5.9 \mathrm{~mm}$, NMV J13938. Antenna 1 flagellum with callynophore. Antenna 2 flagellum with calceoli. Mandible palp article 2 with 10 posterodistal setae. Gnathopod 2 palm with 1 medial robust seta.

Etymology. The specific name is an Australian Aboriginal word meaning "beautiful".

Remarks. Most species of Bamarooka (B. bathycephala, $B$. dinjerra, B. endota and B. kimbla) have ventrally tapered eyes. Bamarooka endota and $B$. dinjerra are very similar. They differ in the basis of pereopod 5, which is almost round in B. endota and has a sloped proximal posterior shoulder in $B$. dinjerra.

Although B. endota and B. bathycephala both occur in Bass Strait in similar depth range, the two species have not been taken in the same samples. Bamarooka bathycephala is found most often on clean sand bottoms whereas $B$. endota is most often found on muddy sand.

Habitat. Bamarooka endota occurs on fine and coarse sediments.

Distribution. Southeastern Australia, from central NSW to Bass Strait; 40-175 m depth.

\section{Bamarooka kimbla n.sp.}

Figs. 55-57

Type material. HoLOTYPE, female, 9.3 mm, AM P36906; 1 PARATYPE, female, $7.8 \mathrm{~mm}$, AM P36907; northeast of Lady Elliott Island, Capricorn Channel, Queensland, Australia, $24^{\circ} 03.7^{\prime} \mathrm{S} 152^{\circ} 49.4^{\prime} \mathrm{E}, 150 \mathrm{~m}$, rubble, large sled dredge, P. Colman, G.Hangay \& S. Keable, 4 July 1984, RV Kimbla, stn 3.

Type locality. Northeast of Lady Elliott Island, Capricorn Channel, Queensland, Australia, $24^{\circ} 03.7^{\prime} \mathrm{S} 152^{\circ} 49.4^{\prime} \mathrm{E}, 150$ m depth.

Description. Based on holotype female, $9.3 \mathrm{~mm}$, AM P36906. Head much deeper than long, anterior margin with notch extended into a slit; rostrum present, cone-shaped; eye present, ventrally tapered. Antenna 1 peduncular article $I$ not ball-shaped proximally, distal margin with small medial spine; peduncular article 2 medium length; flagellum without callynophore, calceoli absent. Antenna 2 flagellum about as long as that of antenna 1 , without calceoli. Mouthpart bundle subconical. Epistome/upper lip with broad mid-anterior bulge (lateral view). Mandible lacinia mobilis a stemmed, distally-cusped blade; accessory setal row with intermediate setae; palp article 2 with 2 posterodistal setae, article 3 without A3-seta. Maxilliped outer plate with distal margin smooth, medial margin without notch.

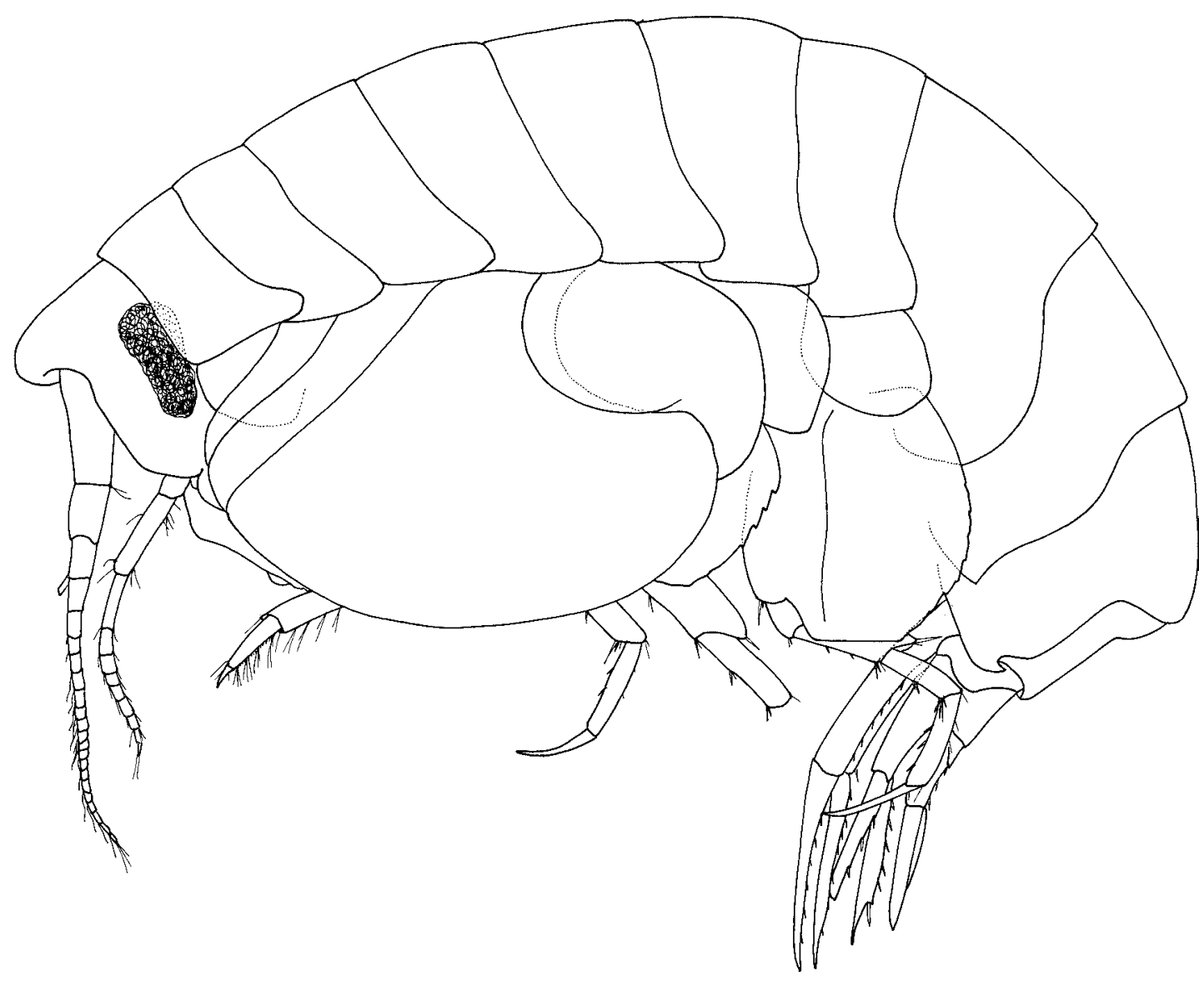

Figure 55. Bamarooka kimbla n.sp., paratype female, 7.8 mm, AM P36907, Capricorn Channel, Queensland. 


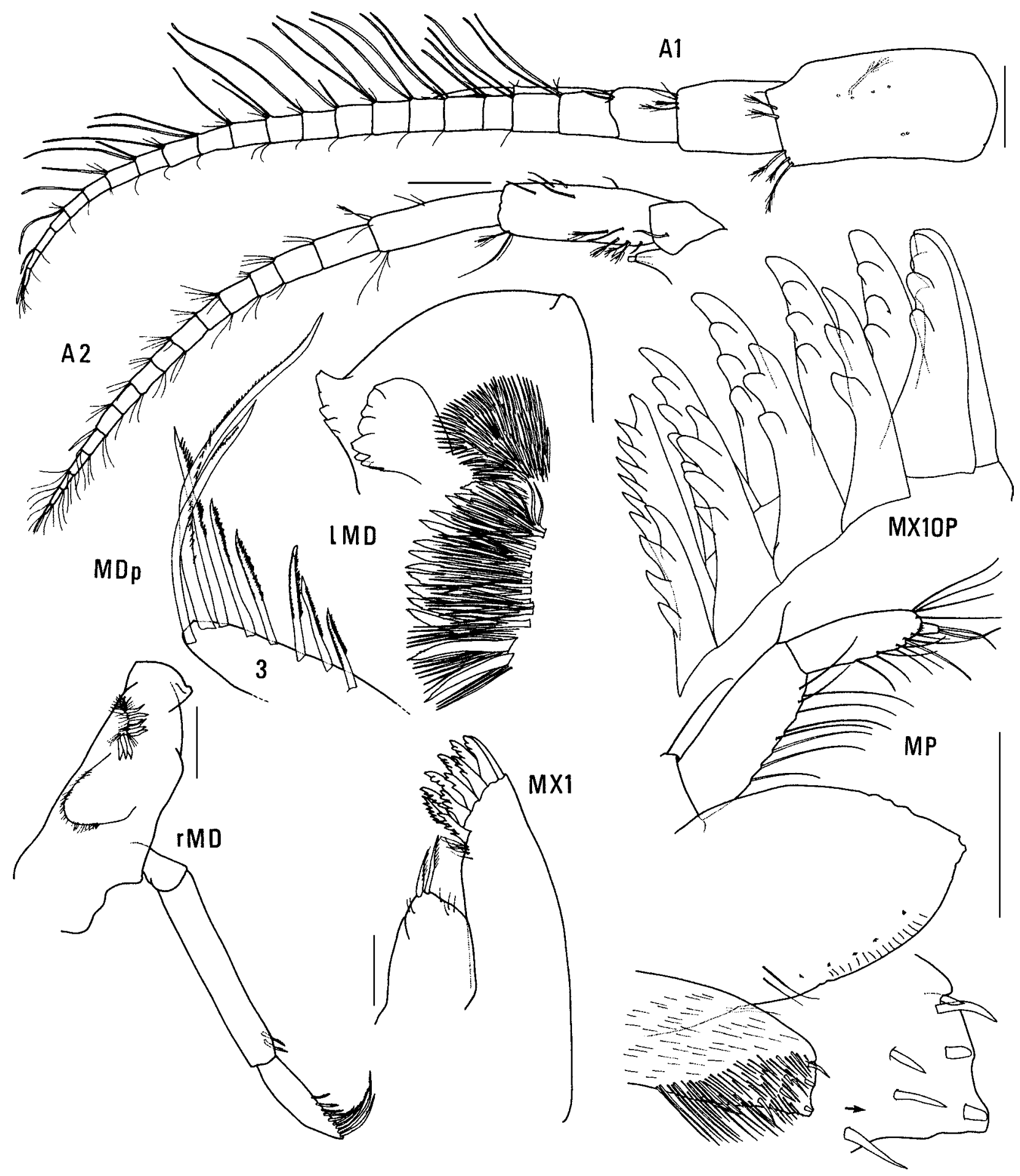

Figure 56. Bamarooka kimbla n.sp., holotype female, 9.3 mm, AM P36906, Capricorn Channel, Queensland. Scales represent $0.1 \mathrm{~mm}$.

Gnathopod 1 carpus subequal in length to propodus; propodus, posterior margin without robust setae. Gnathopod 2 palm acute, with no lateral robust setae, 4 medial robust setae. Pereopods 3 and 4 merus and carpus without setal fringe. Pereopod 4 coxa with anterior margin slightly obtuse, posterior margin rounded, anteroventral corner rounded.
Pereopods 5-7 with distal articles elongate, dactyli long and slender. Pereopod 5 basis expanded posteriorly, rounded with sloping posteroproximal shoulder. Pereopod 7 basis rounded posteriorly, posteroventral corner rounded, posteroventral margin straight.

Figure 57. Bamarooka kimbla n.sp., holotype female, 9.3 mm, AM P36906, Capricorn Channel, Queensland. Scales for G1,2, P5-7 represent $0.5 \mathrm{~mm}$; remainder represent $0.1 \mathrm{~mm}$. 


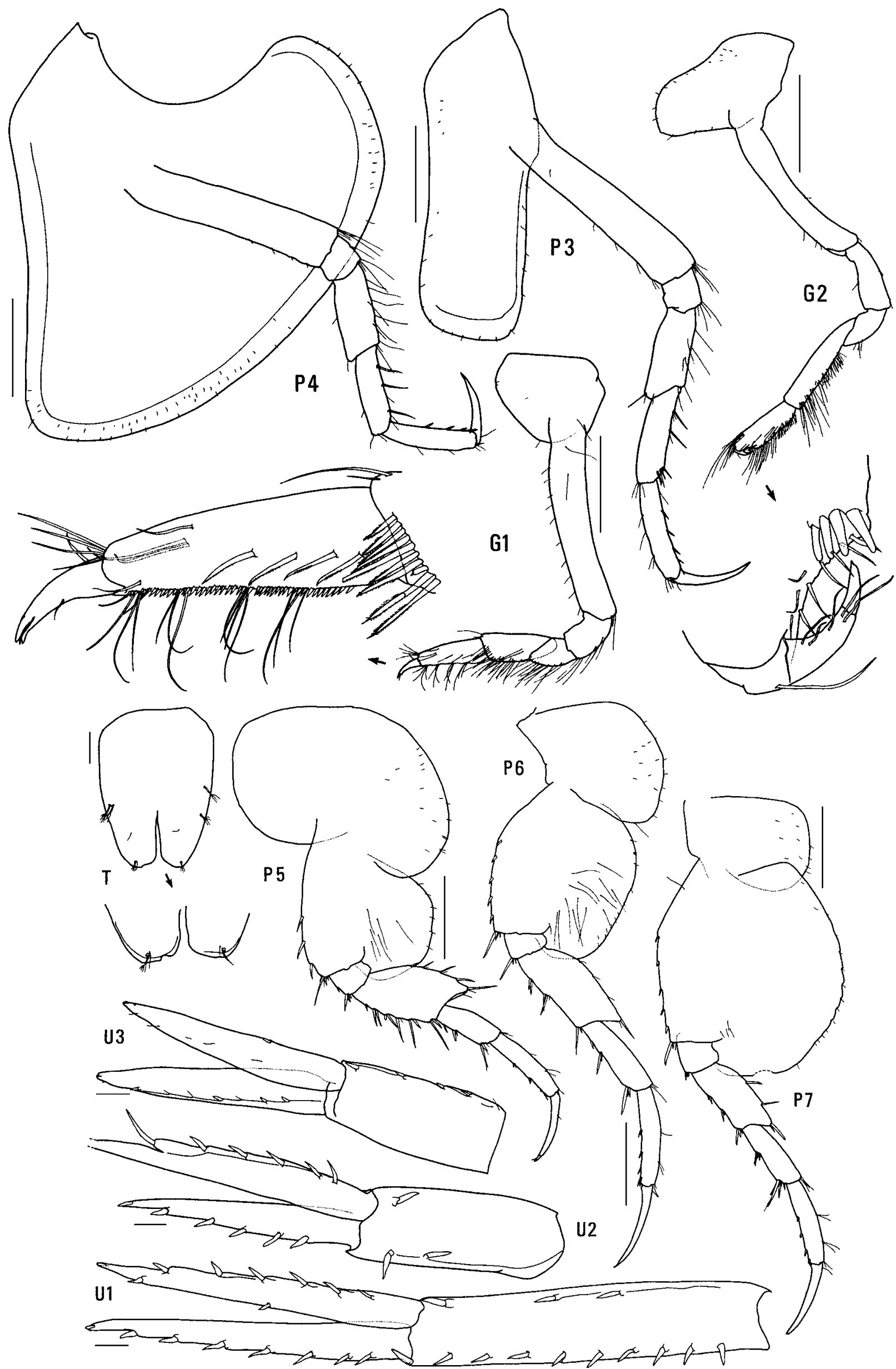


Epimeron 3 posterior margin smooth, with notch well above rounded posteroventral corner. Uropod 1 peduncle dorsolateral margin with 10 robust setae; outer ramus without large spines between robust setae. Uropod 2 inner ramus moderately constricted. Uropod 3 rami lanceolate; without plumose setae; outer ramus 1-articulate. Telson moderately cleft (about $32 \%$ ).

Etymology. The specific name is taken from the research vessel Kimbla, which collected the type material.

Remarks. Most species of Bamarooka (B. bathycephala, $B$. dinjerra, B. endota and B. kimbla) have ventrally tapered eyes. Bamarooka kimbla differs from all of these species in the cone-shaped rostrum.

Distribution. Northeastern Australia; 150 m depth.

\section{Bamarooka tropicalis n.sp.}

Figs. 58-60

Type material. HoLOTYPE, female, $6.0 \mathrm{~mm}$, ovigerous (5 eggs), AM P37074; 1 PARATYPE, male, 3.9 mm, AM P37075; 5 PARATYPES, AM P37076; northwest of Port Hedland, North West Shelf, Western Australia, eastern Indian Ocean, $19^{\circ} 29.2^{\prime} \mathrm{S} 118^{\circ} 52.5^{\prime} \mathrm{E}$ to $19^{\circ} 29.5^{\prime} \mathrm{S} 118^{\circ} 52.3^{\prime} \mathrm{E}, 40-41 \mathrm{~m}$, epibenthic sled, 28 June 1983, FRV Soela, CSIRO stn NWS 03.B8.S. 5 PARATYPES, AM P37077, same locality, $19^{\circ} 29.6^{\prime} \mathrm{S} 118^{\circ} 52.2^{\prime} \mathrm{E}$ to $19^{\circ} 29.4^{\prime} \mathrm{S} 118^{\circ} 52.8^{\prime} \mathrm{E}, 38 \mathrm{~m}$, epibenthic sled, 30 August 1983, CSIRO stn NWS 04.B8.S. 6 PARATYPES, AM P37078, same locality, 19²9.4'S $118^{\circ} 52.5^{\prime} \mathrm{E}$ to $19^{\circ} 30.0^{\prime} \mathrm{S} 118^{\circ} 51.6^{\prime} \mathrm{E}, 38 \mathrm{~m}$, epibenthic sled, 25 October 1983, FRV Soela, CSIRO NWS stn 05.D8.S. 10 PARATYPES, NMV J7667, North West Shelf, Western Australia, eastern Indian Ocean, $19^{\circ} 39^{\prime} \mathrm{S} 116^{\circ} 22^{\prime} \mathrm{E}$ to $19^{\circ} 39^{\prime} \mathrm{S} 116^{\circ} 43^{\prime} \mathrm{E}, 46 \mathrm{~m}$, bryozoans, G.C.B. Poore \& H.M. Lew Ton, 7 June 1983, FRV Soela, stn NWA-33.

\footnotetext{
Additional material. WeSTERn AUSTRALIA: 19 specimens, AM P37079 to P37087, from 9 CSIRO North West Shelf stations close to type locality, 36-40 m, dredged, June-October 1983. NORTHERN TERRITORY: 1 specimen, AM P58075, S side of New Year Island, Arafura Sea, $10^{\circ} 54^{\prime} \mathrm{S}$ $133^{\circ} 02^{\prime} \mathrm{E}, 14 \mathrm{~m}$, hydroids, G.C.B. Poore, 14 October 1982, stn NT-20. 2 specimens, AM P58076, same locality, 14 m, alga Padina, J.K. Lowry, 14 October 1982, stn NT-25. 1 specimen, AM P58077, same locality, 14 m, rubble, J.K. Lowry, 14 October 1982, stn NT-26. 1 specimen, AM $\mathrm{P} 37088$, patch reefs at NW end of McCluer Island, $11^{\circ} 02^{\prime} \mathrm{S} 132^{\circ} 58^{\prime} \mathrm{E}, 8$ $\mathrm{m}$, red algae and sediment, J.K. Lowry, 16 October 1982, stn NT-38. 2 specimens, AM P58078, S end of McCluer Island, Arafura Sea, $11^{\circ} 06 ' \mathrm{~S}$ $133^{\circ} 00^{\prime} \mathrm{E}, 8 \mathrm{~m}$, coral rubble, G.C.B. Poore, 17 October 1982, stn NT-55. 1 specimen, AM P58079, same locality, $8 \mathrm{~m}$, red algae ?Champsia, J.K. Lowry, 17 October 1982, stn NT-58. 14 specimens, AM P37089, same locality, 8 m, Acropora base, P. Horner, 17 October 1982, stn NT-59. 3 specimens, AM P58080, same locality, $8 \mathrm{~m}$, coral rubble with encrusting invertebrates, J.K. Lowry, 17 October 1982, stn NT-60. 2 specimens, AM P37090, same locality, $8 \mathrm{~m}$, Seriatopora hystrix, J.K. Lowry, 17 October 1982, stn NT-61. 6 specimens, AM P37091, W of East Point, north end of Fannie Bay, Darwin, $11^{\circ} 24.5^{\prime} \mathrm{S} 130^{\circ} 48.5^{\prime} \mathrm{E}, 8-10 \mathrm{~m}$, encrusting ascidian Didemnum psammodes, J.K. Lowry, 26 October 1982, stn NT-97. 3 specimens, AM P58081, same locality, 8-10 m, red alga, J.K. Lowry, 26 October 1982, stn NT-103. 1 specimen, AM P18172, Port Darwin, [approx. $12^{\circ} 27^{\prime} \mathrm{S} 130^{\circ} 50^{\prime} \mathrm{E}$ ], W.E.J. Paradice on HMAS Geranium. QUEENSLAND: 1 specimen, AM P37092, fringing reef between Bird Islet and South Island, Lizard Island, $14^{\circ} 40^{\prime} \mathrm{S} 145^{\circ} 28^{\prime} \mathrm{E}, 24.4-27.4$
}

m, Halophila, mixed algae and coral rubble, J.K. Lowry, 7 October 1978, stn QLD-21. 4 specimens, AM P37093, same locality, 27.6 m, Halophila, mixed algae and sediment from seagrass beds on reef base, J.K. Lowry \& P.C. Terrill, 9 October 1978, stn QLD-27\&28. 1 specimen, AM P37094, Lizard Island, 3 m, plankton tow in lagoon, J.M. Leis, 17 October 1979, stn JML 17.10.2. 1 specimen, AM P37095, between Lizard Island and Carter Reef, 32-38 m, algae and sediment, epibenthic sled, J.M. Leis, 26 November 1981, stn JML 81/26-1-4.

Type locality. Northwest of Port Hedland, North West Shelf, Western Australia, eastern Indian Ocean, $1^{\circ} 29^{\prime} \mathrm{S} 118^{\circ} 52^{\prime} \mathrm{E}$, 40-41 m depth.

Description. Based on holotype female, $6.0 \mathrm{~mm}$, AM P37074. Head much deeper than long, anterior margin with notch extended into a slit; rostrum absent; eye elongate, reniform. Antenna 1 peduncular article 1 not ball-shaped proximally, distal margin with small medial spine; peduncular article 2 medium length; flagellum without callynophore, calceoli absent. Antenna 2 flagellum about as long as that of antenna 1, without calceoli. Mouthpart bundle subconical. Epistome/upper lip almost straight (lateral view). Mandible lacinia mobilis a stemmed, distallycusped blade; accessory setal row with intermediate setae; palp article 2 without posterodistal setae, article 3 without A3-seta. Maxilliped outer plate with distal margin smooth, medial margin without notch.

Gnathopod 1 carpus shorter than propodus $(0.8 \times)$; propodus, posterior margin without robust setae. Gnathopod 2 palm slightly acute, with no lateral robust setae, 1 medial robust seta. Pereopods 3 and 4 merus and carpus without setal fringe. Pereopod 4 coxa with anterior margin slightly obtuse, posterior margin rounded, anteroventral corner rounded. Pereopods 5-7 with distal articles elongate, dactyli short and stocky. Pereopod 5 basis expanded posteriorly, rounded with sloping posteroproximal shoulder. Pereopod 7 basis rounded posteriorly, posteroventral corner rounded, posteroventral margin curved.

Epimeron 3 posterior margin smooth, with notch well above rounded posteroventral corner. Uropod 1 peduncle dorsolateral margin with 7 robust setae; outer ramus without large spines between robust setae. Uropod 2 inner ramus slightly constricted. Uropod 3 rami lanceolate; without plumose setae; outer ramus 1-articulate. Telson moderately cleft (about 36\%).

Male (sexually dimorphic characters). Based on paratype male, $3.9 \mathrm{~mm}$, AM P37075. Antenna 1 flagellum with callynophore. Mandible palp article 2 with 1 posterodistal seta.

Etymology. The specific name is a reference to the species' distribution in Australia.

Remarks. Bamarooka anomala and B. tropicalis both have elongate, reniform eyes. They are easily distinguished from each other by their fifth pereopods, which have a linear basis in B. anomala and an expanded basis in B. tropicalis.

Habitat. Bamarooka tropicalis has been found with algae, seagrasses, sediments, ascidians, bryozoa and hydroids, but most commonly among corals and coral rubble.

Distribution. Northern Australia, from North West Shelf off Western Australia to northern Great Barrier Reef, Queensland; 3-40 m depth. 


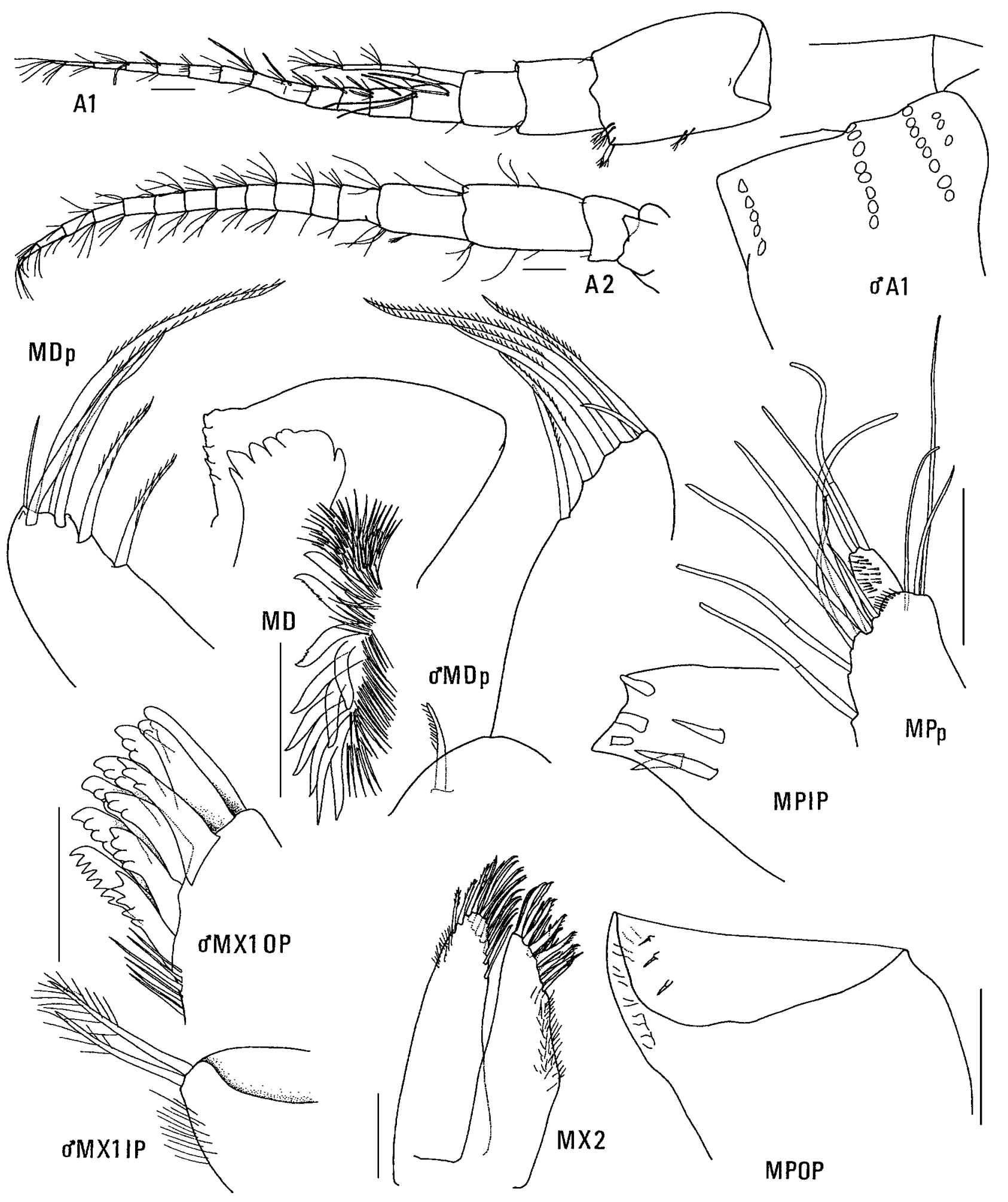

Figure 58. Bamarooka tropicalis n.sp., holotype female, $6.0 \mathrm{~mm}$, AM P37074; paratype male, 3.9 mm, AM P37075; North West Shelf, Western Australia. Scales for A1, 2, MX2, MPOP represent $0.1 \mathrm{~mm}$; remainder represent $0.05 \mathrm{~mm}$. 


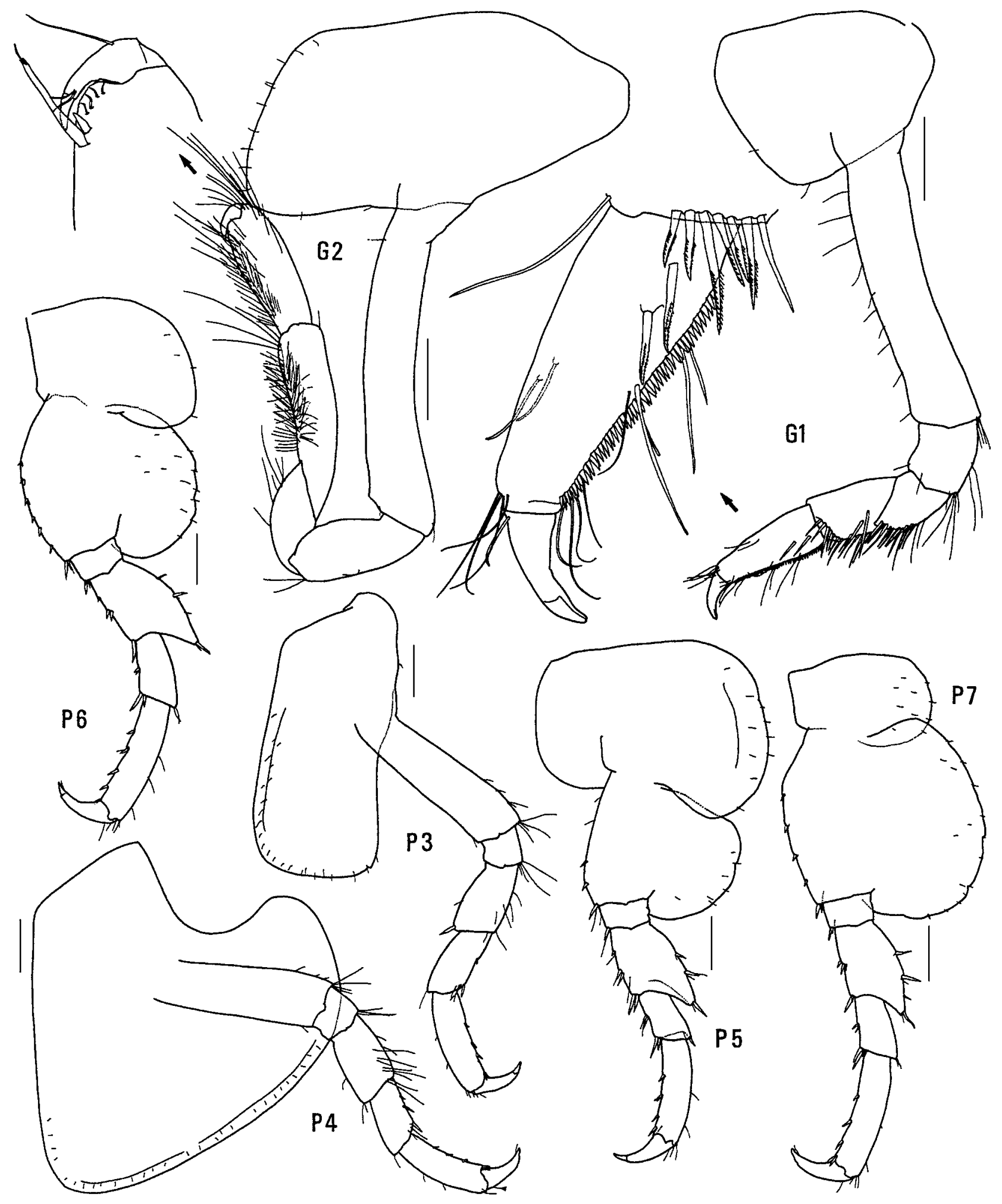

Figure 59. Bamarooka tropicalis n.sp., holotype female, 6.0 mm, AM P37074, North West Shelf, Western Australia. Scales represent $0.2 \mathrm{~mm}$. 


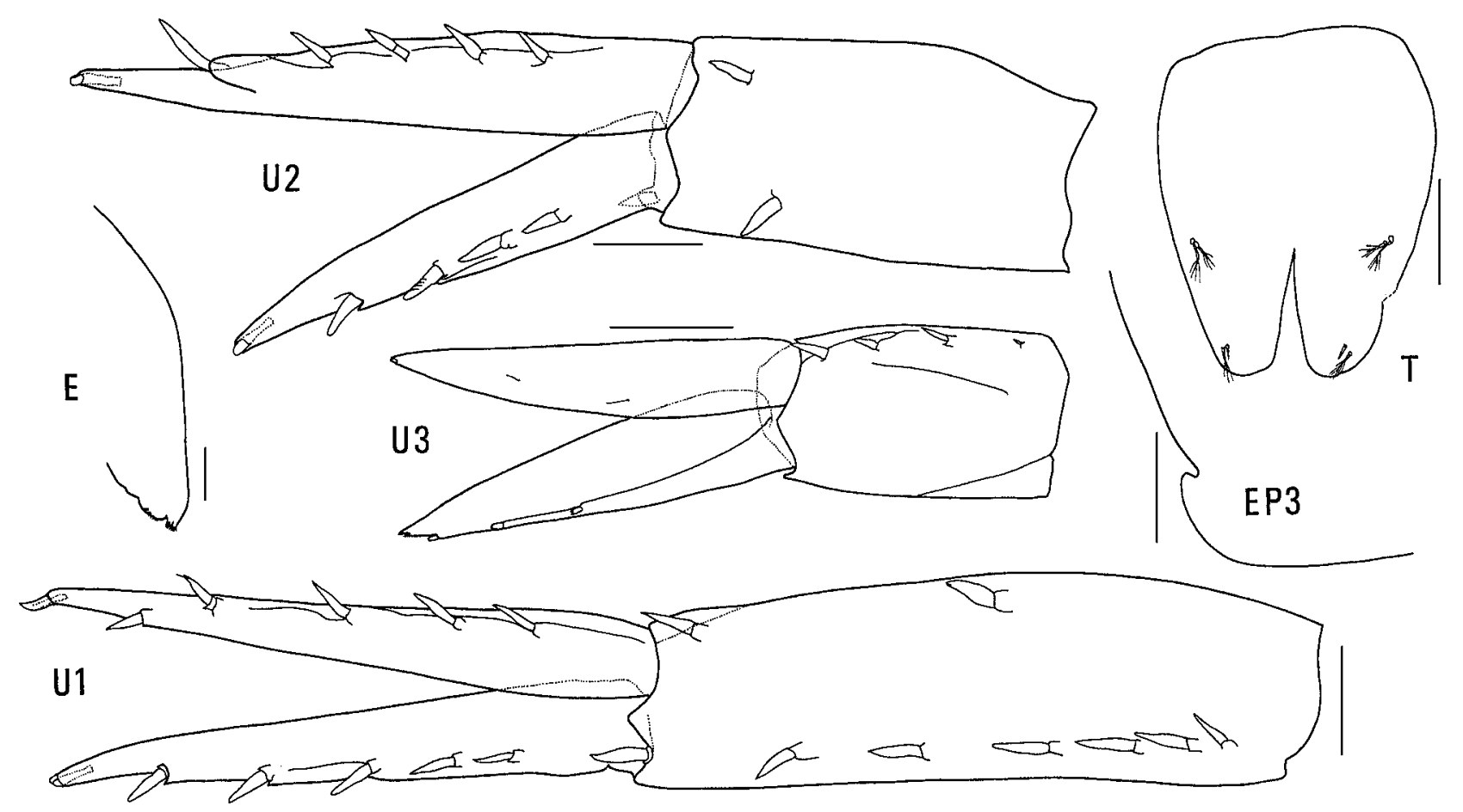

Figure 60. Bamarooka tropicalis n.sp., holotype female, 6.0 mm, AM P37074, North West Shelf, Western Australia. Scales represent $0.1 \mathrm{~mm}$.

\section{Erikus Lowry \& Stoddart}

Erikus Lowry \& Stoddart, 1987: 1303.

Diagnosis. Mouthpart bundle subconical. Mandible palp article 3 with proximal A3-seta. Pereopod 4 coxa with anterior and posterior margins subparallel. Pereopods 5-7 with distal articles elongate. Uropod 3 rami lanceolate; with plumose setae in male and female; outer ramus 2 -articulate.

Type species. Erikus dahli Lowry \& Stoddart, 1987, by original designation.

Species composition. Erikus contains one species: E. dahli Lowry \& Stoddart, 1987.

Remarks. When Lowry \& Stoddart (1987) described Erikus dahli they recorded the uropod 3 outer ramus as 1-articulate. Closer examination has shown that a small, spine-like second article is present. None of the Australian species of Amaryllis have a 2-articulate outer ramus on uropod 3 but specimens in several non-Australian literature records of "Amaryllis macrophthalma" clearly do. Material from Patagonia (Stebbing, 1888), Argentina (Alonso, 1987), Mauritius (Ledoyer, 1978), Madagascar (Ledoyer, 1979; 1986) and the Red Sea (Lyons \& Myers, 1991) all have the spine-like article 2 either illustrated or described. Stebbing (1908: South African material) and Schellenberg (1931: South American material) have recorded "Amaryllis macrophthalma" with plumose setae on male pereopods 3 and 4; Stebbing (1908: South African material), K.H. Barnard (1916: South African material), Ledoyer (1979; 1986: material from Madagascar) have recorded "Amaryllis macrophthalma" with pappose setae on uropod 3; and Ledoyer (1986: material from Madagascar) has recorded "Amaryllis macrophthalma" with A3-setae on the mandibular palp. None of these characters occur in any Australian species of

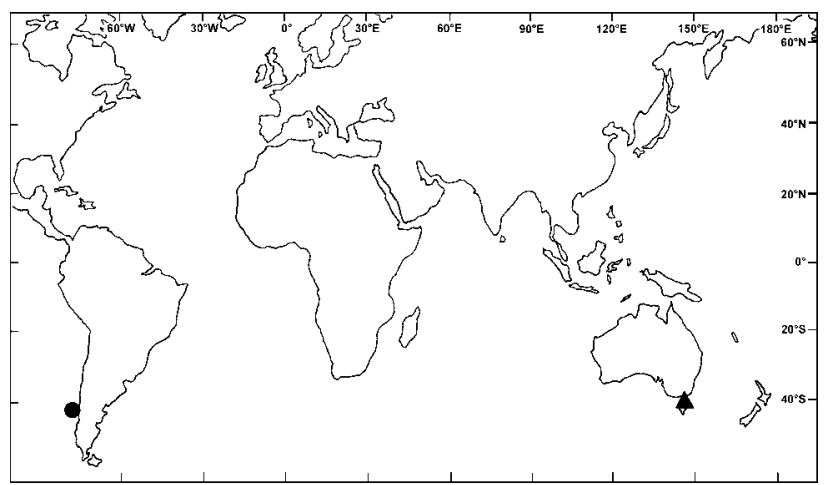

Figure 61. Distribution of genus Erikus; $\boldsymbol{\Delta}$ Distribution of genus Wonga.

Amaryllis, but all three characters occur in Erikus dahli. In our opinion none of these records belong in the genus Amaryllis; they most likely belong in the genus Erikus.

Distribution. Erikus is a shallow-water genus currently known from southern South America, but there is evidence that it also occurs in Africa (see Fig. 61)

\section{Wonga n.gen.}

Diagnosis. Mouthpart bundle conical. Mandible palp article 3 without A3-seta. Pereopod 4 coxa with anterior and posterior margins subparallel. Pereopods 5-7 with distal articles short. Uropod 3 rami clasper-like; without plumose setae in female [male not known]; outer ramus 1-articulate.

Type species. Wonga wonga n.sp.

Species composition. Wonga contains one species: Wonga wonga n.sp. 
Etymology. The genus takes its name from the Australasian Steam Navigation Company's screw steamer Wonga Wonga, introduced as a Bass Strait ferry in 1855 .

Distribution. Southeastern Australia; 140 m depth (see Fig. 61).

\section{Wonga wonga n.sp.}

Figs. 62-65

Type material. HOLOTYPE, female, $6.2 \mathrm{~mm}$, ovigerous (2 eggs), AM P36871; $100 \mathrm{~km}$ northeast of North Point, Flinders Island, eastern Bass Strait, Australia, 38 $52.6^{\prime} \mathrm{S}$ $148^{\circ} 26.5^{\prime} \mathrm{E}, 140 \mathrm{~m}$, fine sand, epibenthic sled, R. Wilson, 15 November 1981, RV Tangaroa, stn BSS 170S.

Type locality. $100 \mathrm{~km}$ northeast of North Point, Flinders Island, eastern Bass Strait, Australia, 38 $8^{\circ} 2.6^{\prime} \mathrm{S} 148^{\circ} 26.5^{\prime} \mathrm{E}$, $140 \mathrm{~m}$, depth.

Description. Based on holotype female, 6.2 mm, AM P36871. Head much deeper than long, anterior margin with notch extended into a slit; rostrum absent; eye present, elongate, reniform. Antenna 1 peduncular article 1 not ball-shaped proximally, distal margin with small medial spine; peduncular article 2 medium length; flagellum with callynophore, calceoli absent. Antenna 2 flagellum about as long as that of antenna 1, without calceoli. Mouthpart bundle conical. Epistome/upper lip almost straight (lateral view). Mandible lacinia mobilis a stemmed, distally-cusped blade; accessory setal row with intermediate setae; palp article 2 with 2 posterodistal setae, article 3 without A3-seta. Maxilliped outer plate with distal margin smooth, medial margin without notch.
Gnathopod 1 carpus subequal in length to propodus $(0.9 \times)$; propodus, posterior margin without robust setae. Gnathopod 2 palm transverse, without lateral robust setae, with 1 medial robust seta. Pereopods 3 and 4 merus and carpus without setal fringe. Pereopod 4 coxa with anterior and posterior margins subparallel, anteroventral corner rounded. Pereopods 5-7 with distal articles short, dactyls short and stocky. Pereopod 5 basis expanded posteriorly, rounded. Pereopod 7 basis subquadrate, posteroventral corner subquadrate, posteroventral margin straight.

Epimeron 3 posterior margin smooth, with notch well above rounded posteroventral corner. Uropod 1 peduncle dorsolateral margin with 5 robust setae; outer ramus without large spines between robust setae. Uropod 2 inner ramus not constricted. Uropod 3 rami clasper-like; without plumose setae; outer ramus 1 -articulate. Telson moderately cleft (about 32\%).

Etymology. The species takes its name from the Port Jackson tugboat Wonga.

Remarks. The telson is shortened and only slightly cleft in Wonga wonga, and the rami of the third uropods are modified into a clamp-like structure, conditions which might be associated with a sedentary life-style. These modifications make $W$. wonga a distinctive species, but unfortunately nothing is known of its behaviour and little more of its habitat.

Habitat. Wonga wonga is known from only one collection, on a fine sand bottom.

Distribution. Bass Strait, southeastern Australia; 140 m depth.

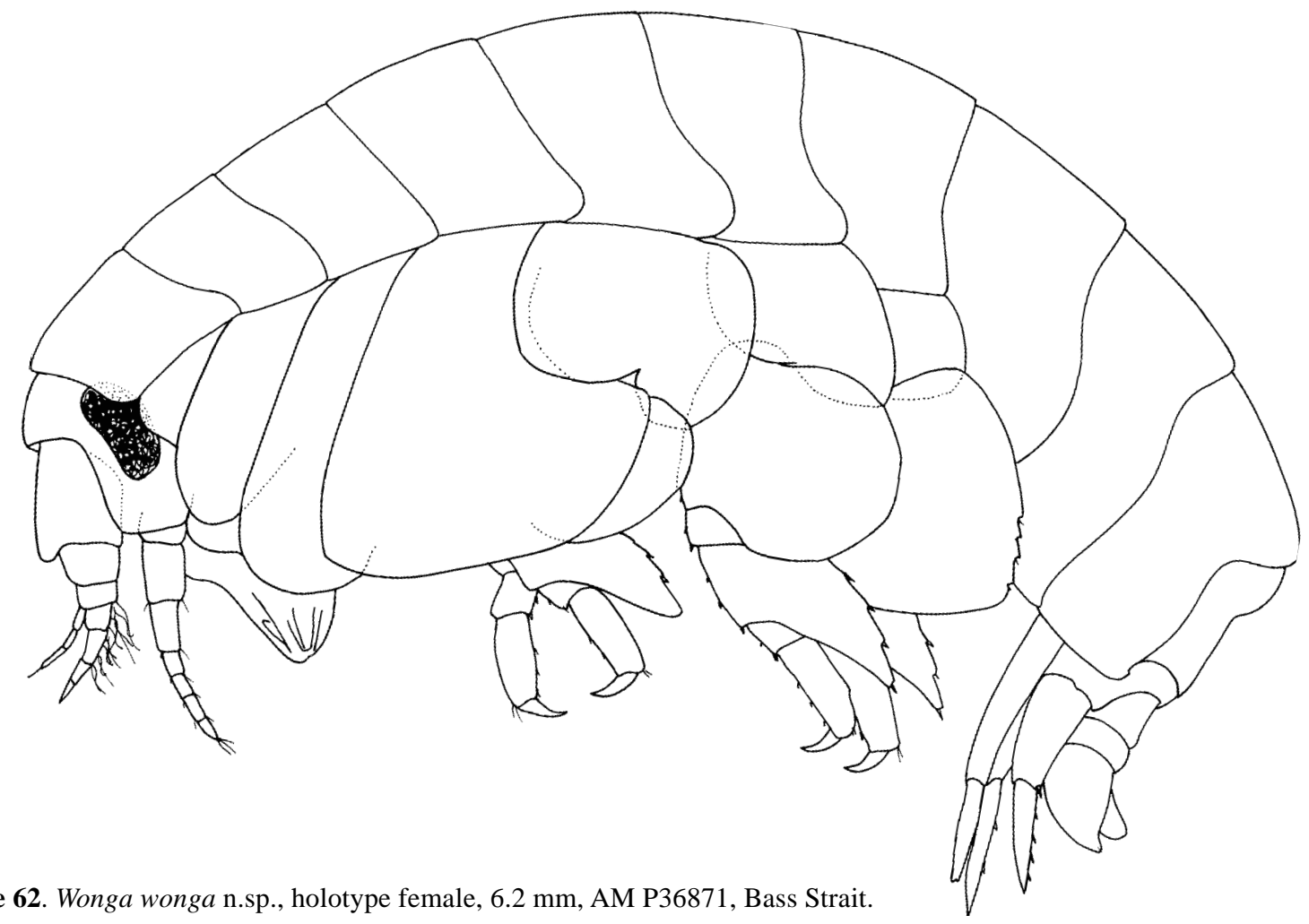

Figure 62. Wonga wonga n.sp., holotype female, 6.2 mm, AM P36871, Bass Strait. 


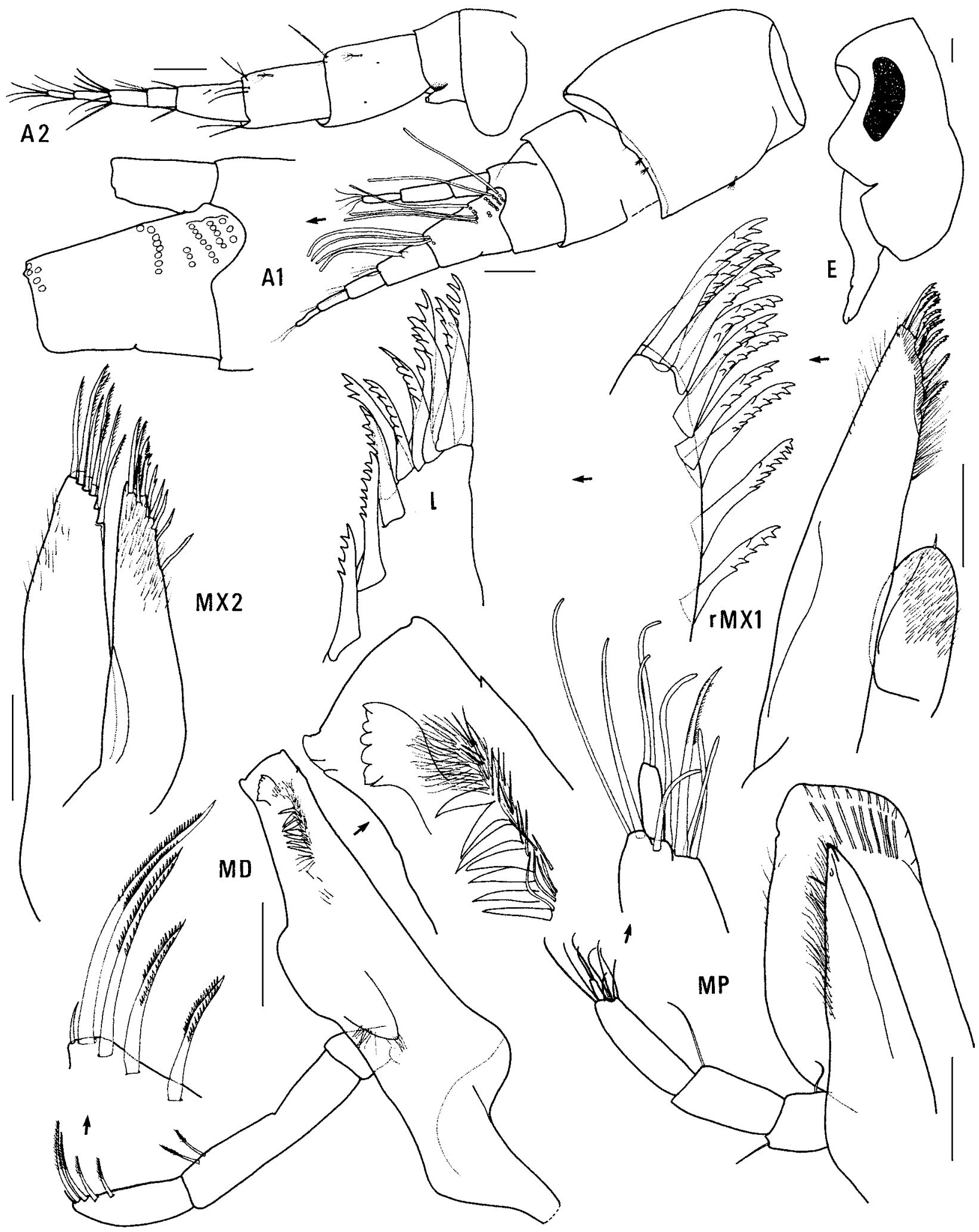

Figure 63. Wonga wonga n.sp., holotype female, $6.2 \mathrm{~mm}$, AM P36871, Bass Strait. Scales represent $0.1 \mathrm{~mm}$. 


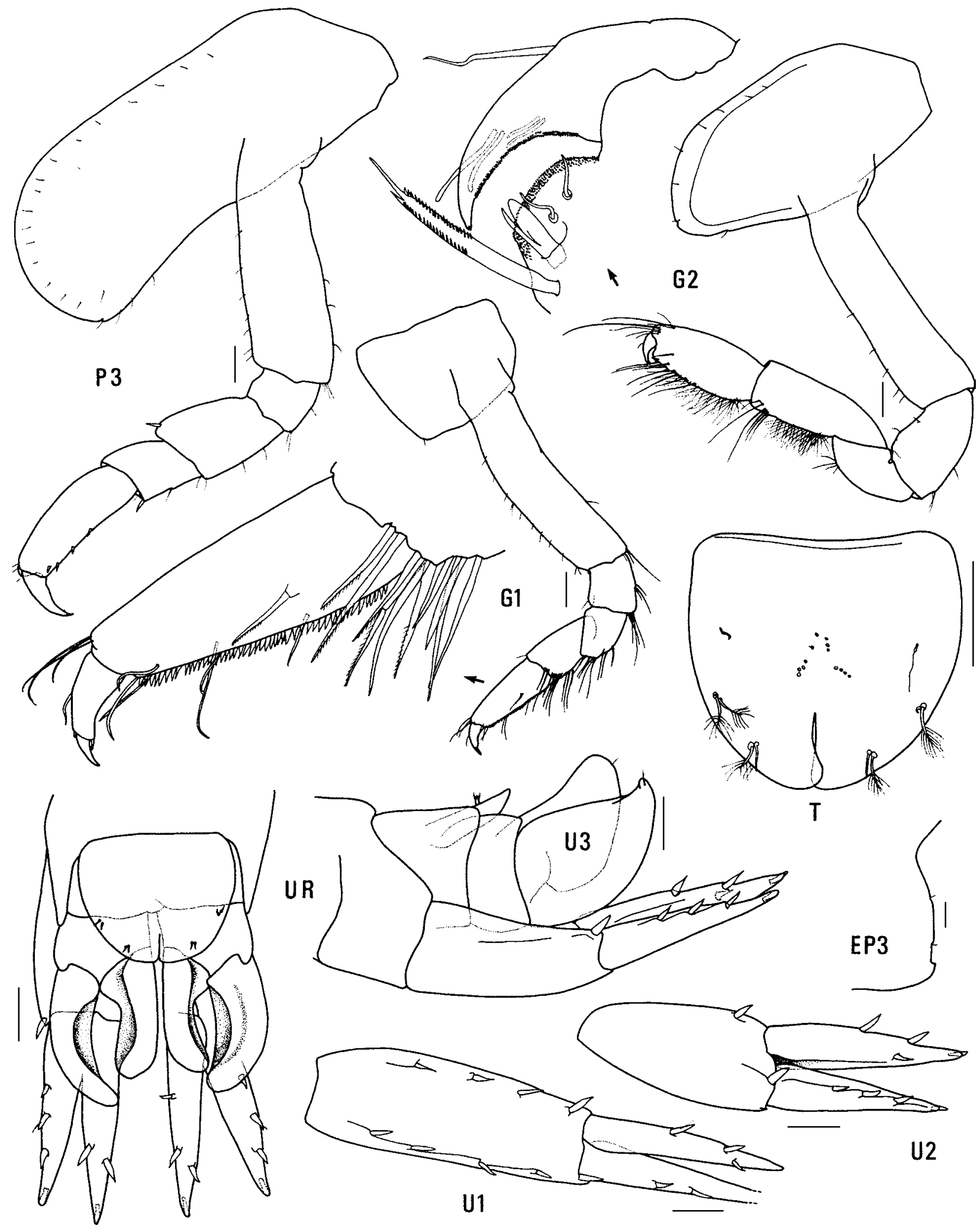

Figure 64. Wonga wonga n.sp., holotype female, $6.2 \mathrm{~mm}$, AM P36871, Bass Strait. Scales represent $0.1 \mathrm{~mm}$. 


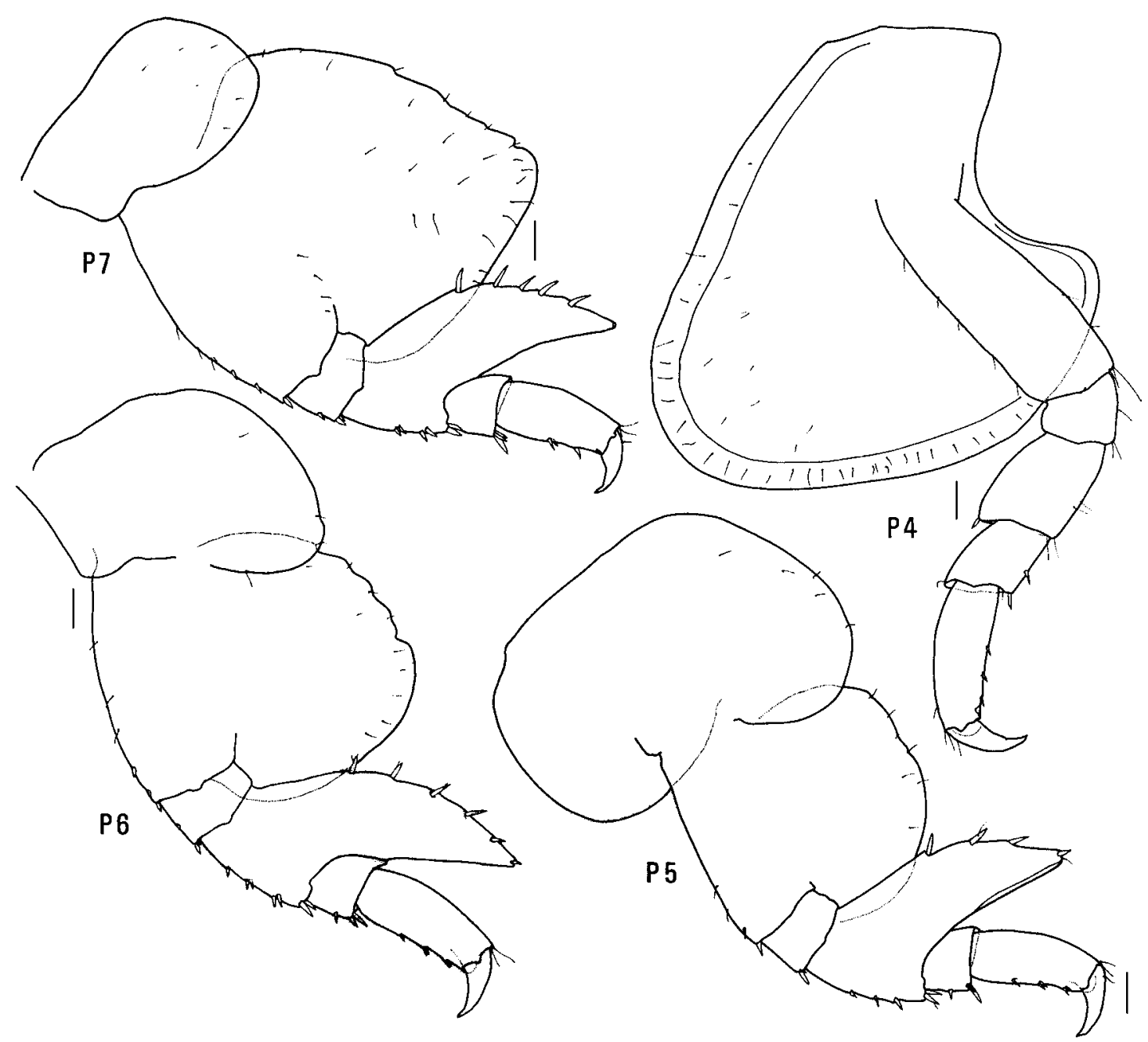

Figure 65. Wonga wonga n.sp., holotype female, $6.2 \mathrm{~mm}$, AM P36871, Bass Strait. Scales represent $0.1 \mathrm{~mm}$.

\section{Vijayiinae n.subfam.}

Diagnosis. Mouthpart bundle subquadrate. Gnathopod 1 propodus with robust setae along posterior margin. Epimeron 3 with notch immediately above posteroventral corner.

Type genus. Vijaya Walker, 1904.

Generic composition. The subfamily contains four genera: Bathyamaryllis Pirlot, 1933; Devo n.gen.; Pseudamaryllis Andres, 1981 and Vijaya Walker, 1904.

Remarks. Vijayiines are mostly a free-living deep-water group. Except for Vijaya tenuipes and one species of Pseudamaryllis, the vijayiines have been found in the deep seas of the North and South Atlantic Oceans, the Red Sea and western South Pacific Ocean.

\section{Bathyamaryllis Pirlot}

Bathyamaryllis Pirlot, 1933: 123.-K.H. Barnard, 1940: 441.-J.L. Barnard, 1969: 328.-Barnard \& Karaman, 1991: 470.

Diagnosis. Head with rostrum anteriorly rounded or truncated; eye reniform or oval. Antenna 1 peduncular article 1 ball-shaped proximally; peduncular article 2 medium length or long; callynophore absent in female, present in male. Antenna 2 flagellum about as long as that of antenna 1 in female, longer than body in male [where known]. Mandible palp article 3 with proximal A3-seta in male [where known], without proximal A3-seta in female. Pereopod 4 coxa with anterior and posterior margins subparallel or with anterior margin slightly obtuse, posterior margin straight. Uropod 3 rami with plumose setae in male [where known], without plumose setae in female; outer ramus 1-articulate or 2-articulate.

Type species. Bathyamaryllis perezii Pirlot, 1933, by original designation.

Species composition. Bathyamaryllis contains 5 species: B. haswelli (Stebbing, 1888), B. kapala n.sp., B. ouvea Lowry \& Stoddart, 1994, B. perezii Pirlot, 1933 and $B$. pulchellus (Bonnier, 1896).

Remarks. Within the vijayiine group Devo and Bathyamaryllis are probably sister taxa. Both have the unusual proximal ballshaped peduncular article 1 on antenna 1 .

The deepwater genera Bathyamaryllis and Devo are the most widespread amaryllidid genera with species in the Atlantic and the Indo-West Pacific. The Indo-West Pacific species of Bathyamaryllis (B. kapala, B. ouvea and B. perezii) all have a moderately constricted inner ramus on uropod 2 and a 2-articulate outer ramus on uropod 3; the two North Atlantic species (B. haswelli and B. pulchellus) 
have only a slightly constricted inner ramus on uropod 2 and a 1-articulate uropod 3. These differences suggest two distinct species groups within the genus.

Mature males of Bathyamaryllis species are not well known. Males have never been recorded for B. haswelli or $B$. perezii. The only male known of our new species $B$. kapala is immature. Stephensen (1923: 44) reported a male of $B$. pulchellus in which antenna 2 was "twice as long as antenna 1", but had no calceoli, and setae were present on article 2 of the mandibular palp. The $7.7 \mathrm{~mm}$ male of $B$. ouvea illustrated in Lowry \& Stoddart (1994, fig. 7), is immature. The $8 \mathrm{~mm}$ male of their fig. 10 had both antennae 2 broken; antenna 1 and the remaining articles of antenna 2 had no calceoli; it did have a well-developed callynophore on antenna 1 , brush setae on the peduncle of antenna 2 , numerous setae on articles 2 and 3 of the mandibular palp, a brush of setae on merus and carpus of pereopods 3 and 4 , and plumose setae on the rami of uropod 3 .
Distribution. Western and eastern North Atlantic Ocean, Indonesia, eastern Australia and western South Pacific Ocean; 120-1919 m depth (see Fig. 66).

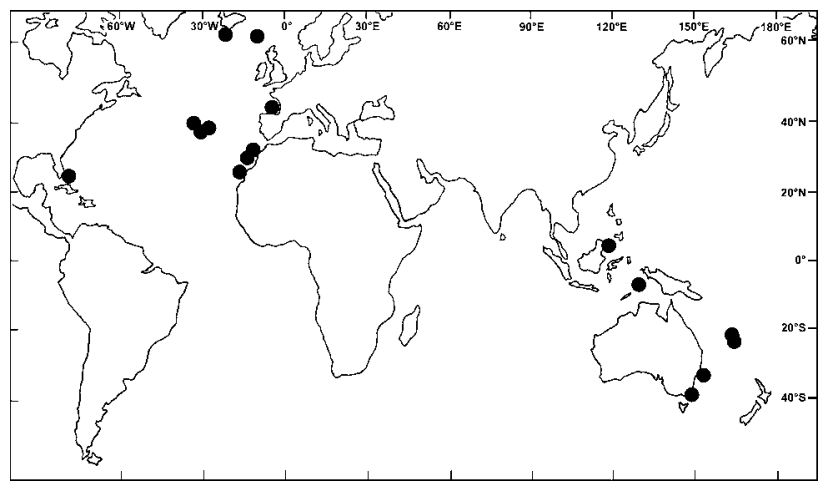

Figure 66. Distribution of genus Bathyamaryllis.

\section{Key to species of Bathyamaryllis}

1 Pereopod 4 coxa with anterior margin slightly obtuse, posterior margin straight

Pereopod 4 coxa anterior and posterior margins subparallel ............................................ 3

2 Head with rostrum anteriorly rounded. Gnathopod 1 carpus longer

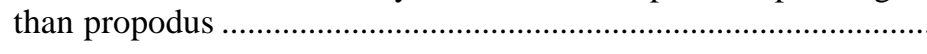

Head with rostrum anteriorly truncated. Gnathopod 1 carpus

subequal to propodus

3 Head anterior margin with a notch only. Gnathopod 1 carpus longer than propodus B. haswelli

_ Head anterior margin with a notch extended into a slit. Gnathopod 1 carpus subequal to propodus..... 4

4 Gnathopod 2 palm acute. Pereopod 4 coxa with anteroventral corner subquadrate B. pulchellus Gnathopod 2 palm transverse. Pereopod 4 coxa with anteroventral corner rounded B. perezii

\section{Bathyamaryllis kapala n.sp.}

Figs. 67-69

Type material. HoLOTYPE, female, $11.5 \mathrm{~mm}$, ovigerous (16 eggs), AM P36868, southeast of Broken Bay, New South Wales, Australia, $33^{\circ} 37^{\prime} \mathrm{S} 152^{\circ} 04^{\prime} \mathrm{E}$ to $33^{\circ} 39^{\prime} \mathrm{S} 152^{\circ} 02^{\prime} \mathrm{E}$, 897-924 m, dredge, R.T. Springthorpe, 10 December 1980, FRV Kapala, stn K80-20-09. 1 PARATYPE, immature, AM P36869, E of Broken Bay, NSW, Australia, 3331'S $152^{\circ} 08^{\prime} \mathrm{E}$ to $33^{\circ} 33^{\prime} \mathrm{S} 152^{\circ} 07^{\prime} \mathrm{E}, 914 \mathrm{~m}$, dredge, R.T. Springthorpe, 10 December 1980, FRV Kapala, stn K8020-08. 3 PARATYPES ( 2 females and 1 immature male), AM P36870, E of Broken Bay, NSW, Australia, 33 $30^{\circ} \mathrm{S}$ $152^{\circ} 09^{\prime} \mathrm{E}$ to $33^{\circ} 33^{\prime} \mathrm{S} 152^{\circ} 11^{\prime} \mathrm{E}, 922-1015 \mathrm{~m}$, beam trawl, R.T. Springthorpe, 12 February 1986, FRV Kapala, stn K8601-08. 1 PARATYPE, female, AM P58308, E of Broken Bay, NSW, Australia, $33^{\circ} 30^{\prime} \mathrm{S} 152^{\circ} 12^{\prime} \mathrm{E}$ to $33^{\circ} 33^{\prime} \mathrm{S} 152^{\circ} 09^{\prime} \mathrm{E}$, 1036-1049 m, dredge, R.T. Springthorpe, 12 February 1986, FRV Kapala, stn K86-01-10. 5 PARATYPES (1 ovigerous female, 4 juveniles), NMV J48797, $54 \mathrm{~km}$ ESE of Nowra, NSW, Australia, $34^{\circ} 52.72^{\prime} \mathrm{S} 151^{\circ} 15.04^{\prime} \mathrm{E}, 996 \mathrm{~m}$, mud, fine sand, fine shell, epibenthic sled, G.C.B. Poore et al., 22 October 1988, RV Franklin, stn SLOPE 53. 1 PARATYPE,
NMV J48796, $76 \mathrm{~km} \mathrm{~S} \mathrm{of} \mathrm{Point} \mathrm{Hicks,} \mathrm{Victoria,} \mathrm{Australia,}$ $38^{\circ} 29.33^{\prime} \mathrm{S} 149^{\circ} 19.98^{\prime} \mathrm{E}, 1840 \mathrm{~m}$, sandy mud, fine shell, epibenthic sled, G.C.B. Poore et al., 26 October 1988, RV Franklin, stn SLOPE 69.

Type locality. Southeast of Broken Bay, NSW, Australia, Tasman Sea, $33^{\circ} 37^{\prime} \mathrm{S} 152^{\circ} 04^{\prime} \mathrm{E}$ to $33^{\circ} 39^{\prime} \mathrm{S} 152^{\circ} 02^{\prime} \mathrm{E}, 896-$ $924 \mathrm{~m}$ depth.

Description. Based on holotype female, $11.5 \mathrm{~mm}$, AM P36868. Head much deeper than long, anterior margin with notch extended into a slit; rostrum present, anteriorly rounded; eye present or apparently absent, elongate, reniform. Antenna 1 peduncular article 1 ball-shaped proximally (slightly), distal margin with large medial spine; peduncular article 2 long, length $2 \times$ breadth; flagellum without callynophore, calceoli absent. Antenna 2 flagellum about as long as that of antenna 1. Mouthpart bundle subquadrate. Mandible lacinia mobilis a stemmed distolaterally cusped blade; accessory setal row with intermediate setae; palp article 2 without posterodistal setae, article 3 without A3-seta. Maxilliped outer plate with distal margin serrate (minutely), medial margin without notch. 


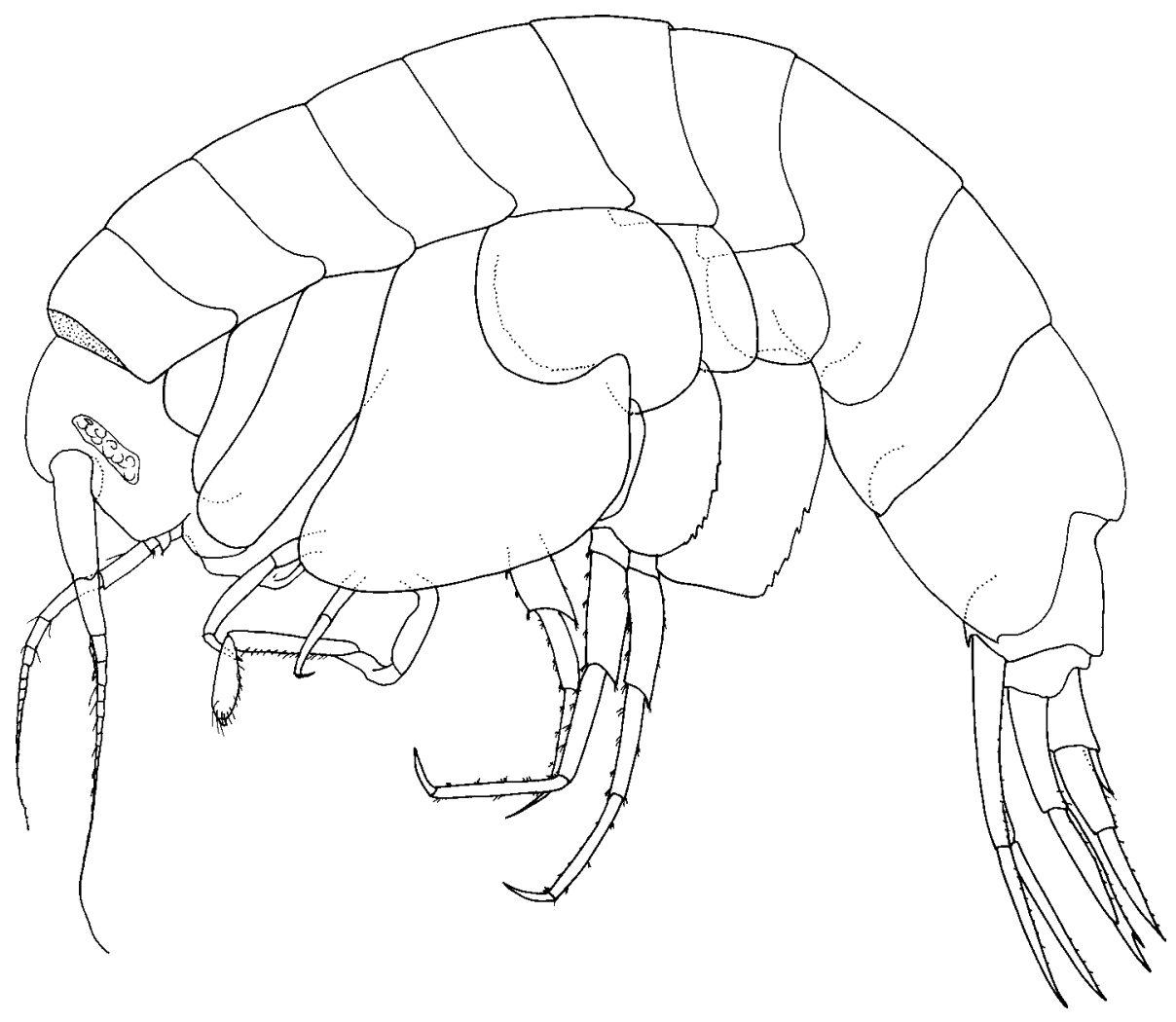

Figure 67. Bathyamaryllis kapala n.sp., holotype female, 11.5 mm, AM P36868, southeast of Broken Bay, NSW.

Gnathopod 1 carpus longer than propodus $(1.3 \times)$; propodus, posterior margin with robust setae. Gnathopod 2 palm acute, with 1 lateral robust seta, 1 medial robust seta. Pereopods 3 and 4 merus and carpus without setal fringe. Pereopod 4 coxa with anterior margin slightly obtuse, posterior margin straight, anteroventral corner rounded. Pereopods 5-7 with distal articles elongate, dactyls short and slender. Pereopod 5 basis expanded posteriorly, rounded. Pereopod 7 basis subrectangular, posteroventral corner subquadrate, posteroventral margin straight.

Epimeron 3 posterior margin smooth, with notch immediately above acute posteroventral corner. Uropod 1 peduncle dorsolateral margin with 12 robust setae; outer ramus without large spines between robust setae. Uropod 2 inner ramus moderately constricted. Uropod 3 rami lanceolate; without plumose setae; outer ramus 2-articulate. Telson moderately cleft (about 35\%).

Etymology. Named for the former New South Wales Fisheries Research Vessel, the FRV Kapala, which made many valuable collections of marine invertebrates off the coast of New South Wales.

Remarks. All three Indo-West Pacific species of Bathyamaryllis are very similar. Bathyamaryllis kapala can be distinguished from B. perezii by the shape of coxa 4 , which has a slightly obtuse anterior margin and a straight posterior margin in B. kapala, but subparallel anterior and posterior margins in $B$. perezii. It can be distinguished from $B$. ouvea by the shape of the rostrum, which is rounded in B. kapala and truncated in B. ouvea.

Habitat. Bathyamaryllis kapala has been collected from sand and mud bottoms but its habitat is generally not known.

Distribution. Southeastern Australia, Tasman Sea; 896$1840 \mathrm{~m}$ depth.

\section{Devo n.gen.}

Diagnosis. Head with rostrum cone-shaped; eye, when present, oval. Antenna 1 peduncular article 1 ball-shaped proximally; peduncular article 2 long; callynophore absent in female, present in male. Antenna 2 flagellum about as long as that of antenna 1 in female, longer than body in male. Mandible palp article 3 without proximal A3-seta. Pereopod 4 coxa with anterior margin slightly obtuse, posterior margin rounded or with anterior margin slightly obtuse, posterior margin straight. Uropod 3 rami with plumose setae in male, with or without plumose setae in female; outer ramus 1-articulate or 2-articulate.

\section{Type species. Devo grahami n.sp.}

Species composition. Devo contains four species: $D$. conocephala (K.H. Barnard, 1925), D. dubuc n.sp., D. grahami n.sp. and D. rostrata (Chevreux, 1911).

Etymology. The name Devo is an allusion to a recent American music group of the same name, whose members wore peculiar cone-shaped head coverings.

Remarks. Devo forms a distinctive group of species, distinguished from other vijayiines by their strongly flared fourth coxae and well-developed rostrums. Species in the genus Devo appear to be more adapted for a pelagic lifestyle than are other amaryllidids.

Distribution. Eastern North and South Atlantic Oceans, southwestern Indian Ocean and western South Pacific Ocean; 500-1840 m depth (see Fig. 70). 


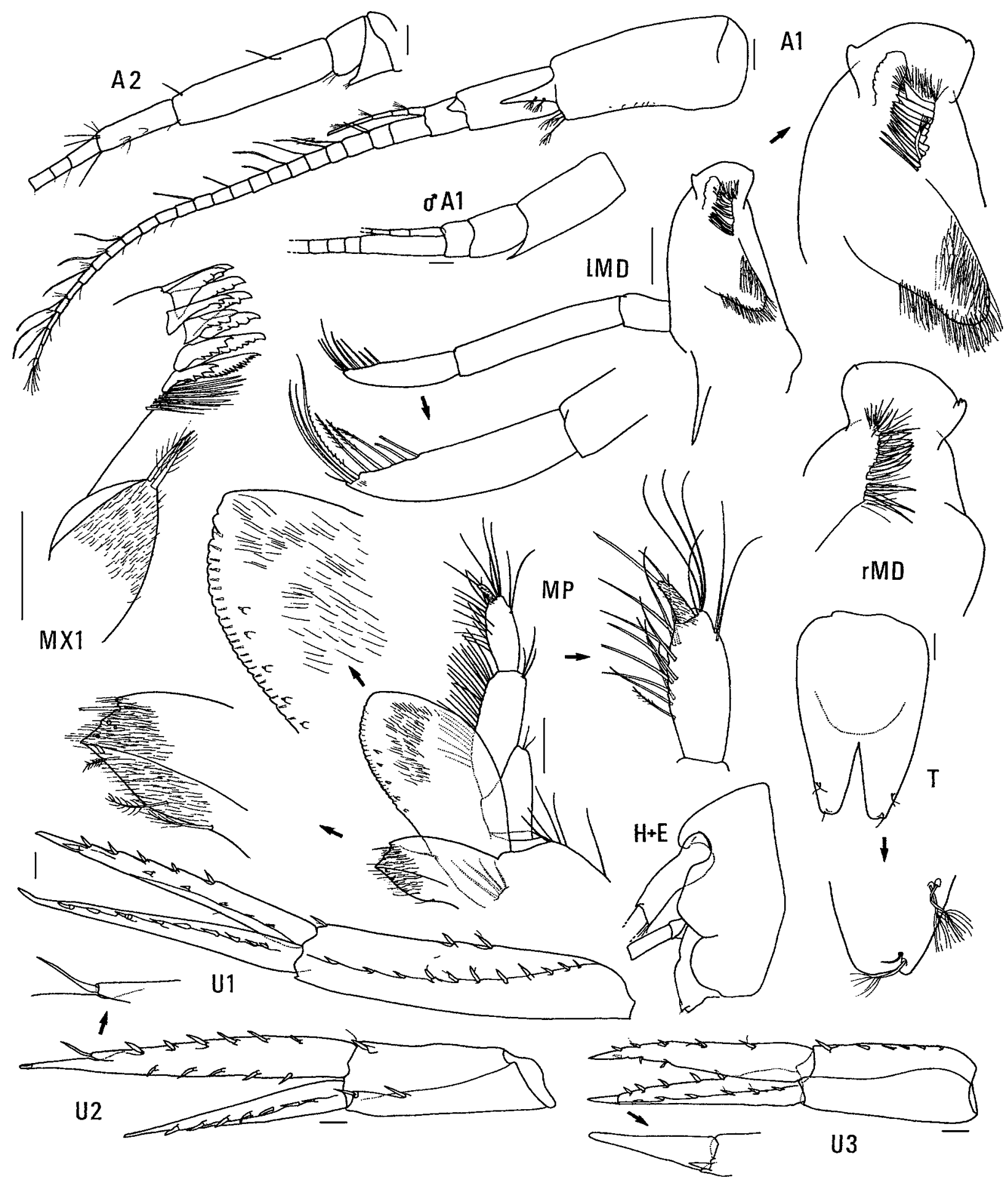

Figure 68. Bathyamaryllis kapala n.sp., holotype female, $11.5 \mathrm{~mm}$, AM P36868, southeast of Broken Bay, NSW; paratype immature male, $7.5 \mathrm{~mm}$, AM P36870, east of Broken Bay, NSW. Scales represent $0.1 \mathrm{~mm}$. 


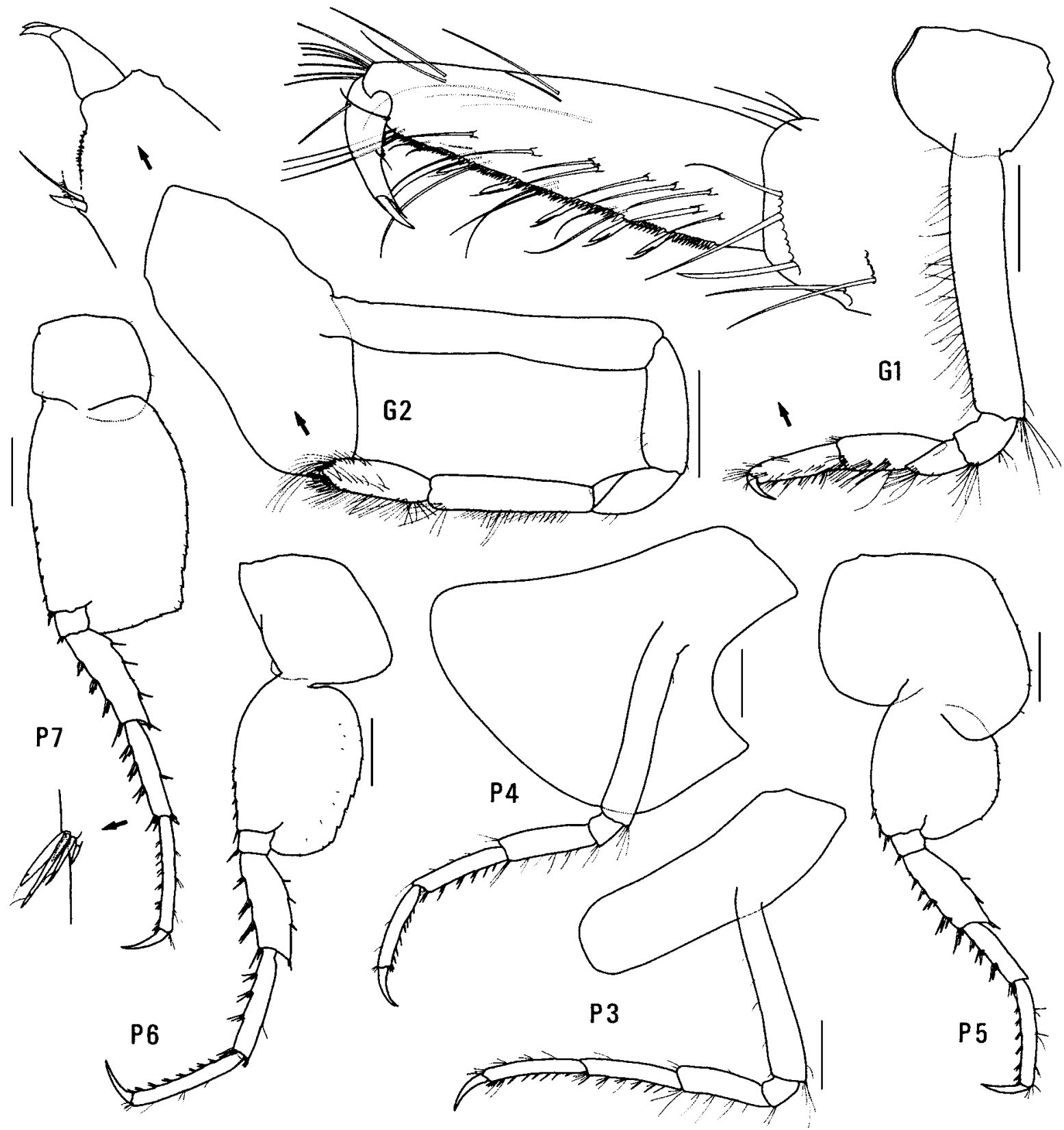

Figure 69. Bathyamaryllis kapala n.sp., holotype female, 11.5 mm, AM P36868, southeast of Broken Bay, NSW. Scales represent $0.5 \mathrm{~mm}$.

\section{Key to species of Devo}

$1 \quad$ Head much deeper than long ...... 2

Head about as deep as long......

D. rostrata

2 Head anterior margin with notch or with notch extended into a slit. Pereopod 4 coxa with anteroventral corner rounded. 3

— Head anterior margin without notch or slit. Pereopod 4 coxa with anteroventral corner subquadrate

D. conocephalus

3 Head anterior margin with notch extended into a slit. Female uropod 3 without plumose setae D. dubuc

Head anterior margin with notch only. Female uropod 3 with plumose setae 


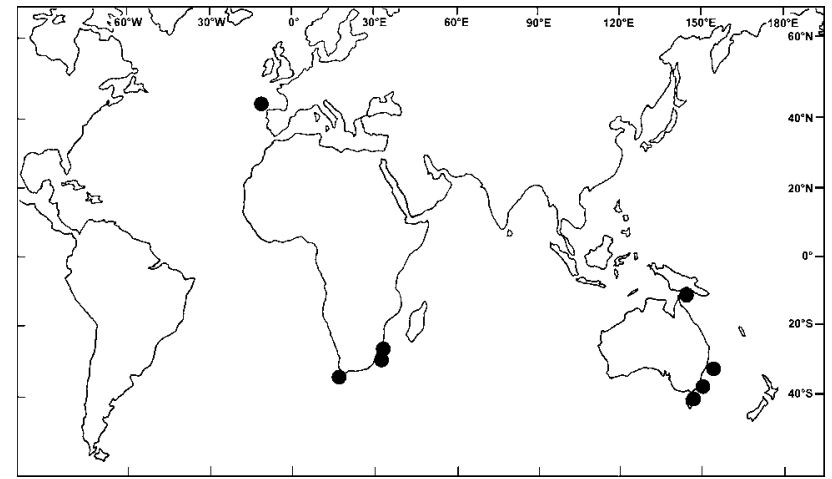

Figure 70. Distribution of genus Devo.

\section{Devo dubuc n.sp.}

Figs. 71, 72

Type material. HOLOTYPE, female, $7.2 \mathrm{~mm}$, with non-setose oostegites, NMV J48795; 1 PARATYPE, male, 7.5 mm, NMV J48794; 1 PARATYPE, male, 7.4 mm, NMV J48793; 10 PARATYPES, NMV J15795; 10 PARATYPES, AM P58963; 48 km east-north-east of Cape Tourville, Tasmania, Australia, $42^{\circ} 00.25^{\prime} \mathrm{S} 148^{\circ} 43.55^{\prime} \mathrm{E}, 1264 \mathrm{~m}$, gravel with lumps of sandy mud aggregate, epibenthic sled, G.C.B. Poore et al., 30 October 1988, RV Franklin, stn SLOPE 81.

Type locality. $48 \mathrm{~km}$ east-north-east of Cape Tourville, Tasmania, Australia, $42^{\circ} 00.25^{\prime} \mathrm{S} 148^{\circ} 43.55^{\prime} \mathrm{E}, 1264 \mathrm{~m}$ depth.

Description. Based on holotype female, $7.2 \mathrm{~mm}$, NMV $\mathrm{J} 48795$. Head much deeper than long, anterior margin with notch extended into a slit; rostrum present, cone-shaped; eye apparently absent. Antenna 1 peduncular article 1 ballshaped proximally, distal margin with well-developed medial spine; peduncular article 2 long, length $4.5 \times$ breadth; flagellum without callynophore, calceoli absent. Antenna 2 flagellum about as long as that of antenna 1, without calceoli. Mouthpart bundle subquadrate. Mandible lacinia mobilis a stemmed distolaterally cusped blade; accessory setal row with intermediate setae; palp article 2 without posterodistal setae, article 3 without A3-seta. Maxilliped outer plate with distal margin smooth, medial margin without notch.

Gnathopod 1 carpus longer than propodus $(1.2 \times)$; propodus, posterior margin with robust setae. Gnathopod 2 palm acute, without lateral robust setae, with 1 medial robust seta. Pereopods 3 and 4 merus and carpus without setal fringe. Pereopod 4 coxa with anterior margin slightly obtuse, posterior margin straight, anteroventral corner subquadrate. Pereopods 5-7 with distal articles elongate, dactyls long and slender. Pereopod 5 basis expanded posteriorly, rounded. Pereopod 7 basis subrectangular, posteroventral corner rounded, posteroventral margin straight.

Epimeron 3 posterior margin smooth, with notch immediately above acute posteroventral corner. Uropod 1 peduncle dorsolateral margin with 11 robust setae; outer ramus without large spines between robust setae. Uropod 2 inner ramus moderately constricted. Uropod 3 rami lanceolate; without plumose setae; outer ramus 2-articulate. Telson moderately cleft (about 40\%).

Male (sexually dimorphic characters). Based on paratype male, $7.5 \mathrm{~mm}$. Antenna 1 flagellum with callynophore, with calceoli. Mandible palp article 2 with 8 posterodistal setae. Pereopods 3 and 4 merus and carpus with setal fringe. Uropod 3 rami with plumose setae.

Etymology. Named for the whaling ship Dubuc, scuttled off Kangaroo Bluff, Bellrieve, in the Derwent River, Tasmania, in November 1808.

Remarks. The main difference between $D$. dubuc and $D$. grahami is the anterior margin of the head, which has a notch extended into a slit in $D$. dubuc but only a simple notch in $D$. grahami. In addition, $D$. dubuc does not have plumose setae on uropod 3 of the female.

Habitat. Devo dubuc is known from only one collection, on a coarse sediment bottom.

Distribution. Southeastern Australia, Tasman Sea; 1264 m depth.

\section{Devo grahami n.sp.}

Figs. 73-75, Plate 1c

Type material. HoLOTYPE, female, $7.7 \mathrm{~mm}$, ovigerous (8 eggs), AM P36872; 1 PARATYPE, male, 9.3 mm, AM P36873; 5 PARATYPES, AM P36874; E of Broken Bay, New South Wales, Australia, $33^{\circ} 31^{\prime} \mathrm{S} 152^{\circ} 08^{\prime} \mathrm{E}$ to $33^{\circ} 33^{\prime} \mathrm{S} 152^{\circ} 07^{\prime} \mathrm{E}, 914 \mathrm{~m}$, dredge, R.T. Springthorpe, 10 December 1980, FRV Kapala, stn K80-20-8. 5 PARATYPES, AM P56969, E of Broken Bay, NSW, Australia, $33^{\circ} 29^{\prime} \mathrm{S} 152^{\circ} 12^{\prime} \mathrm{E}$ to $33^{\circ} 27^{\prime} \mathrm{S} 152^{\circ} 14^{\prime} \mathrm{E}, 1143$ 1200 m, beam trawl, R.T. Springthorpe, 12 February 1986, FRV Kapala, stn K86-01-09. 5 PARATYPES (4 males, 1 female), AM P36875, E of Broken Bay, NSW, Australia, 3330'S $152^{\circ} 12^{\prime} \mathrm{E}$ to $33^{\circ} 33^{\prime} \mathrm{S} 152^{\circ} 09^{\prime} \mathrm{E}, 1036-1049 \mathrm{~m}$, R.T. Springthorpe, 12 February 1986, Kapala, stn K86-01-10. 1 PARATYPE, female, AM P36876, SE of Broken Bay, NSW, Australia, $33^{\circ} 41^{\prime} \mathrm{S} 152^{\circ} 01^{\prime} \mathrm{E}, 1125 \mathrm{~m}$, epibenthic sled, 11 December 1978 , FRV Kapala, stn K78-27-03. 1 PARATYPE, immature male, $7.0 \mathrm{~mm}$, AM P36877, E of Port Jackson, NSW, Australia, $33^{\circ} 48^{\prime} \mathrm{S} 151^{\circ} 52^{\prime} \mathrm{E}, 549 \mathrm{~m}$, sled/dredge, 3 December 1979, FRV Kapala, stn K79-20-02. 2 PARATYPES (1 mature male, 1 immature male), NMV J48792; 54 km ESE of Nowra, NSW, Australia, $34^{\circ} 52.72^{\prime} \mathrm{S} 151^{\circ} 15.04^{\prime} \mathrm{E}, 996 \mathrm{~m}$, mud, fine sand, fine shell, epibenthic sled, G.C.B. Poore et al., 22 October 1988, RV Franklin, stn SLOPE 53.

Additional material. Two specimens, AM P56970, E of Cape York, Australia, Coral Sea, $10^{\circ} 32.72^{\prime} \mathrm{S} 144^{\circ} 12.8^{\prime} \mathrm{E}$, epibenthic sled, $780-795$ m, P. Hutchings et al., 20 August 1988, RV Franklin, stn 06/88-03. 1 specimen + fragment, NMV J48791, S of Point Hicks, Victoria, 38 $21.90^{\prime} \mathrm{S}$ $149^{\circ} 20.00^{\prime} \mathrm{E}, 1000 \mathrm{~m}$, epibenthic sled, G.C.B. Poore et al., 23 July 1986 , RV Franklin, stn SLOPE 32. 1 specimen, NMV J48790, S of Point Hicks, Victoria, $38^{\circ} 19.10^{\prime} \mathrm{S} 149^{\circ} 14.30^{\prime} \mathrm{E}, 600 \mathrm{~m}$, coarse sand, epibenthic sled, M.F. Gomon et al., 24 July 1986, RV Franklin, stn SLOPE 39. 19 specimens, NMV J48789, $76 \mathrm{~km} \mathrm{~S}$ of Point Hicks, Victoria, 38 $29.33^{\circ} \mathrm{S}$ $149^{\circ} 19.98^{\prime} \mathrm{E}, 1840 \mathrm{~m}$, sandy mud, fine shell, epibenthic sled, G.C.B. Poore et al., 26 October 1988, RV Franklin, stn SLOPE 69. 4 specimens, NMV J48788, off Freycinet Peninsula, Tasmania, 42 ${ }^{\circ} 2.20^{\prime} \mathrm{S} 148^{\circ} 38.70^{\prime} \mathrm{E}, 800 \mathrm{~m}$, coarse shelly sand, epibenthic sled, M.F. Gomon et al., 27 July 1986, RV Franklin, stn SLOPE 45. 5 specimens, NMV J48787, off Freycinet Peninsula, Tasmania, $42^{\circ} 0.20^{\prime} \mathrm{S} 148^{\circ} 37.70^{\prime} \mathrm{E}, 720 \mathrm{~m}$, coarse shelly sand, epibenthic sled, M.F. Gomon et al., 27 July 1986, RV Franklin, stn SLOPE 46.

Type locality. East of Broken Bay, NSW, Australia, Tasman Sea, $33^{\circ} 31^{\prime} \mathrm{S} 152^{\circ} 08^{\prime} \mathrm{E}$ to $33^{\circ} 33^{\prime} \mathrm{S} 152^{\circ} 07^{\prime} \mathrm{E}, 914 \mathrm{~m}$ depth.

Description. Based on holotype female, $7.7 \mathrm{~mm}$, AM P36872. Head much deeper than long, anterior margin with notch; rostrum present, cone-shaped; eye present, elongate, 


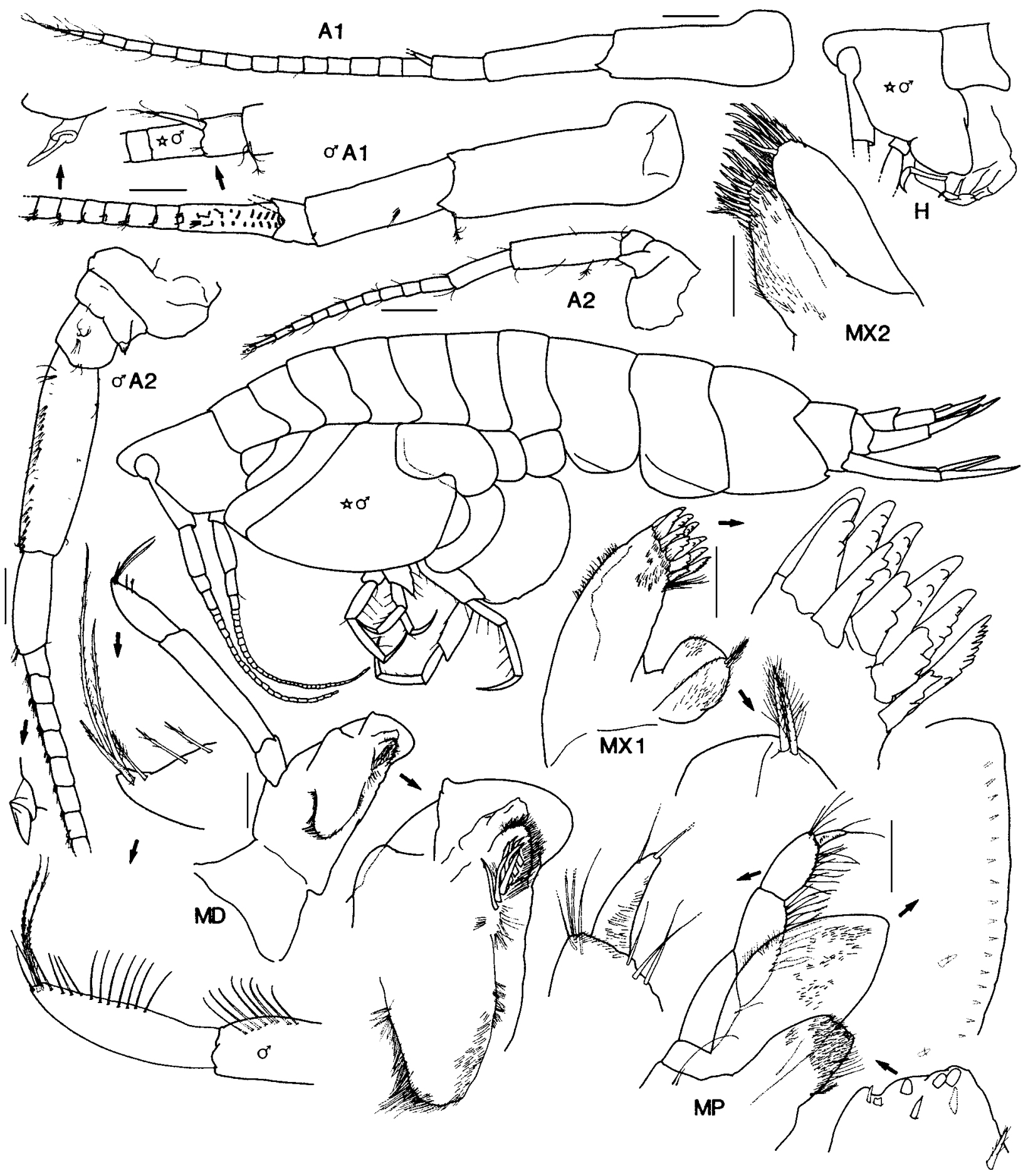

Figure 71. Devo dubuc n.sp., holotype female, $7.2 \mathrm{~mm}$, NMV J48795; paratype male, $7.5 \mathrm{~mm}$, NMV J48794; 弥 paratype immature male, $7.4 \mathrm{~mm}$, NMV J48793; off Cape Tourville, Tasmania. Scales for A1, A2 represent 0.2 $\mathrm{mm}$; remainder represent $0.1 \mathrm{~mm}$.

oval. Antenna 1 peduncular article 1 ball-shaped proximally, distal margin with well-developed medial spine; peduncular article 2 long, length $5 \times$ breadth; flagellum without callynophore, calceoli absent. Antenna 2 flagellum about as long as that of antenna 1, without calceoli. Mouthpart bundle subquadrate. Epistome/upper lip almost straight (lateral view). Mandible lacinia mobilis a stemmed distolaterally cusped blade; accessory setal row with intermediate setae; palp article 2 without posterodistal setae, article 3 without A3-seta. Maxilliped outer plate with distal margin smooth, medial margin without notch.

Gnathopod 1 carpus longer than propodus $(1.3 \times)$; propodus, posterior margin with robust setae. Gnathopod 2 palm acute, without lateral robust setae, 1 medial robust seta. Pereopods 3 and 4 merus and carpus without setal fringe. Pereopod 4 coxa with anterior margin slightly 


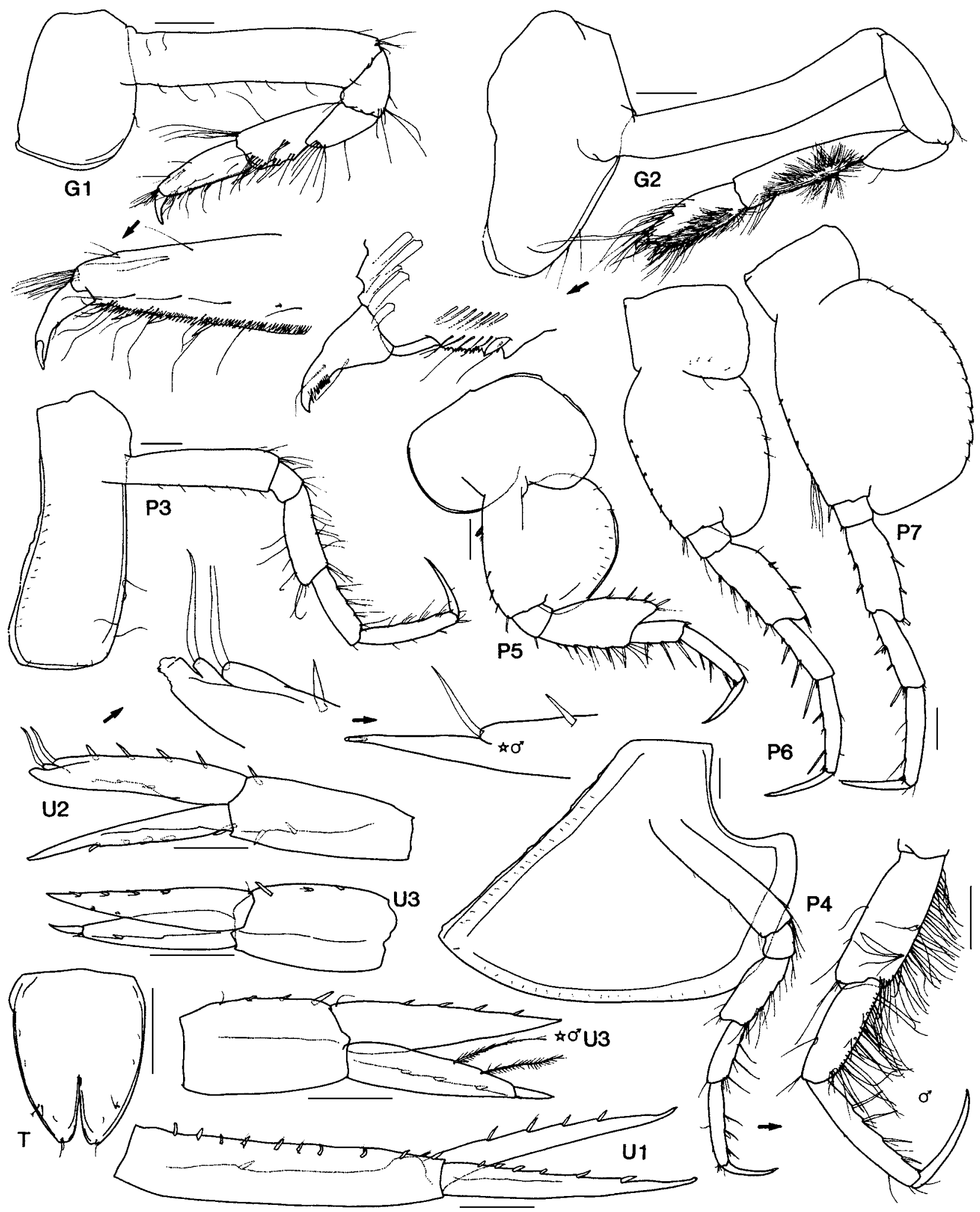

Figure 72. Devo dubuc n.sp., holotype female, $7.2 \mathrm{~mm}$, NMV J48795; paratype male, $7.5 \mathrm{~mm}$, NMV J48794; 论 paratype immature male, $7.4 \mathrm{~mm}$, NMV J48793; off Cape Tourville, Tasmania. Scales represent $0.2 \mathrm{~mm}$.

Figure 73. Devo grahami n.sp., whole animal: paratype immature male, $7.0 \mathrm{~mm}, \mathrm{AM}$ P36877; o : paratype mature male, $9.3 \mathrm{~mm}$, AM P36873; remainder: holotype female, 7.7 mm, AM P36872; east of Broken Bay, NSW. Scales represent $0.1 \mathrm{~mm}$. 


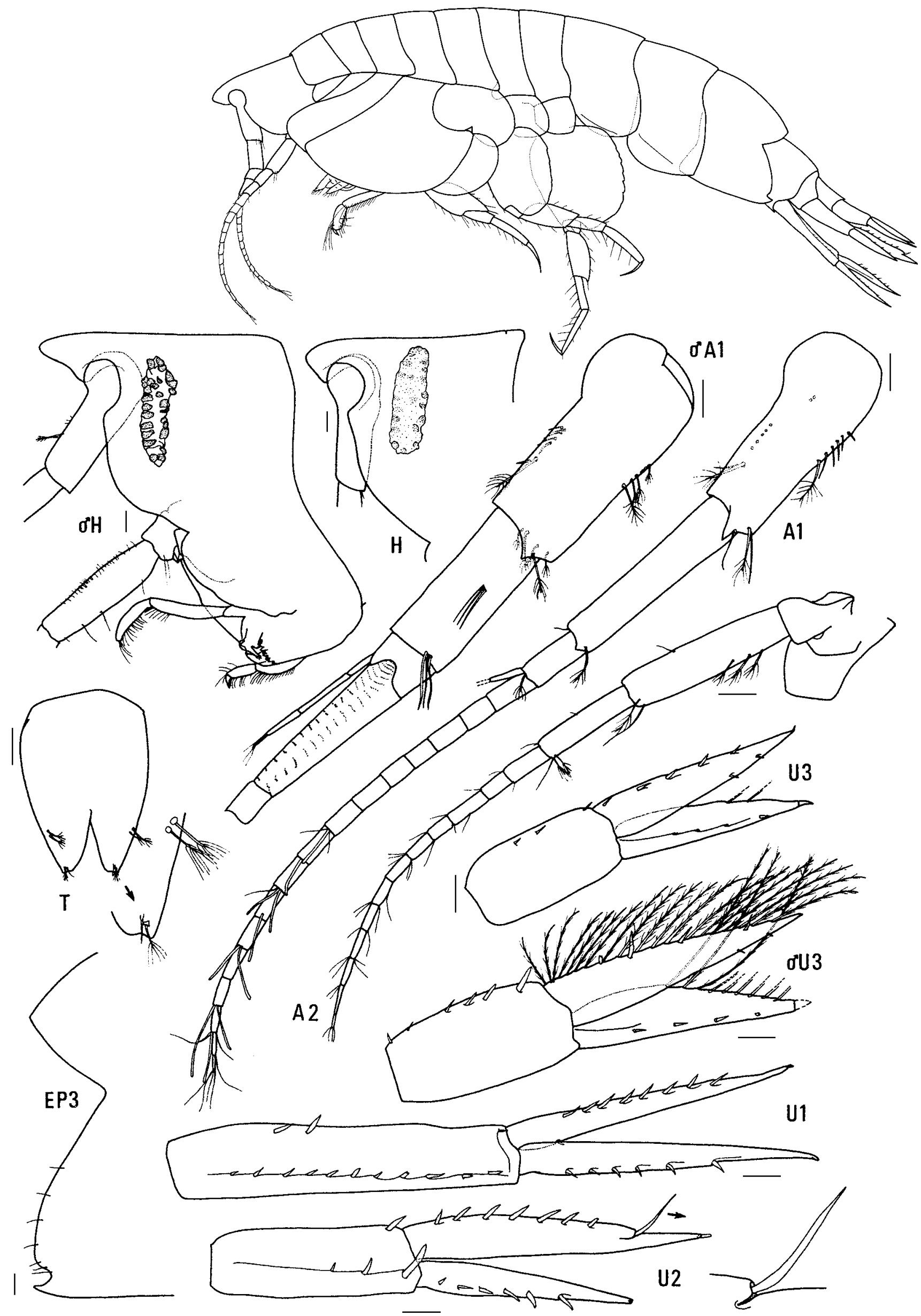




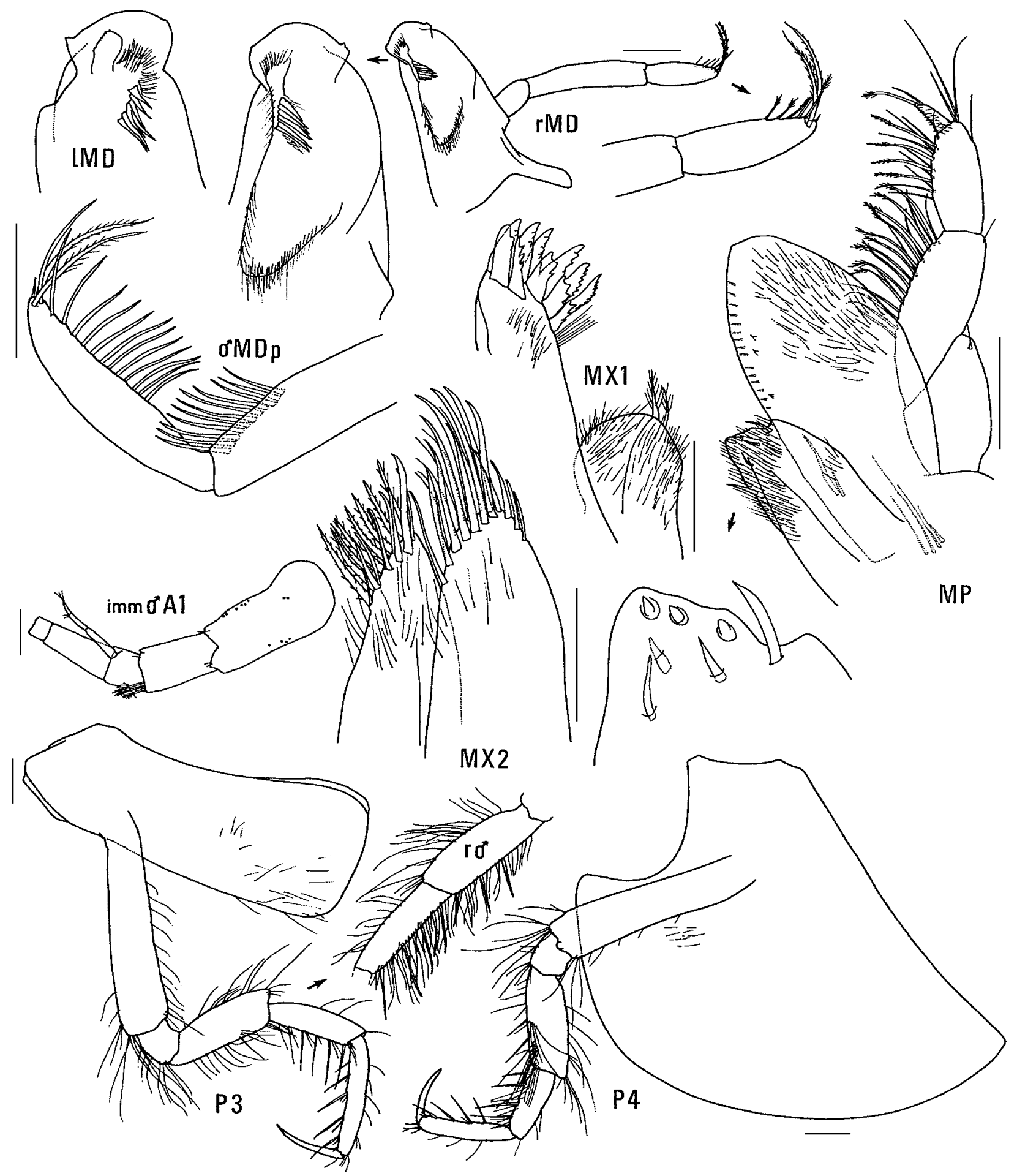

Figure 74. Devo grahami n.sp., holotype female, 7.7 mm, AM P36872; paratype mature male, 9.3 mm, AM P36873; paratype immature male, $7.0 \mathrm{~mm}$, AM P36877; east of Broken Bay, NSW. Scales for P3, 4 represent $0.2 \mathrm{~mm}$; remainder represent $0.1 \mathrm{~mm}$.

obtuse, posterior margin straight, anteroventral corner subquadrate. Pereopods 5-7 with distal articles elongate, dactyls long and slender. Pereopod 5 basis expanded posteriorly, rounded. Pereopod 7 basis subrectangular, posteroventral corner rounded, posteroventral margin curved.

Epimeron 3 posterior margin smooth, with notch immediately above acute posteroventral corner. Uropod 1 peduncle dorsolateral margin with 12 robust setae; outer ramus without large spines between robust setae. Uropod 2 inner ramus moderately constricted. Uropod 3 rami lanceolate; with a few plumose setae; outer ramus 2articulate. Telson slightly cleft (about 30\%).

Male (sexually dimorphic characters). Based on paratype male, $9.3 \mathrm{~mm}$, AM P36873. Antenna 1 flagellum with callynophore, with calceoli. Mandible palp article 2 with 11 posterodistal setae. Pereopods 3 and 4 merus and carpus 


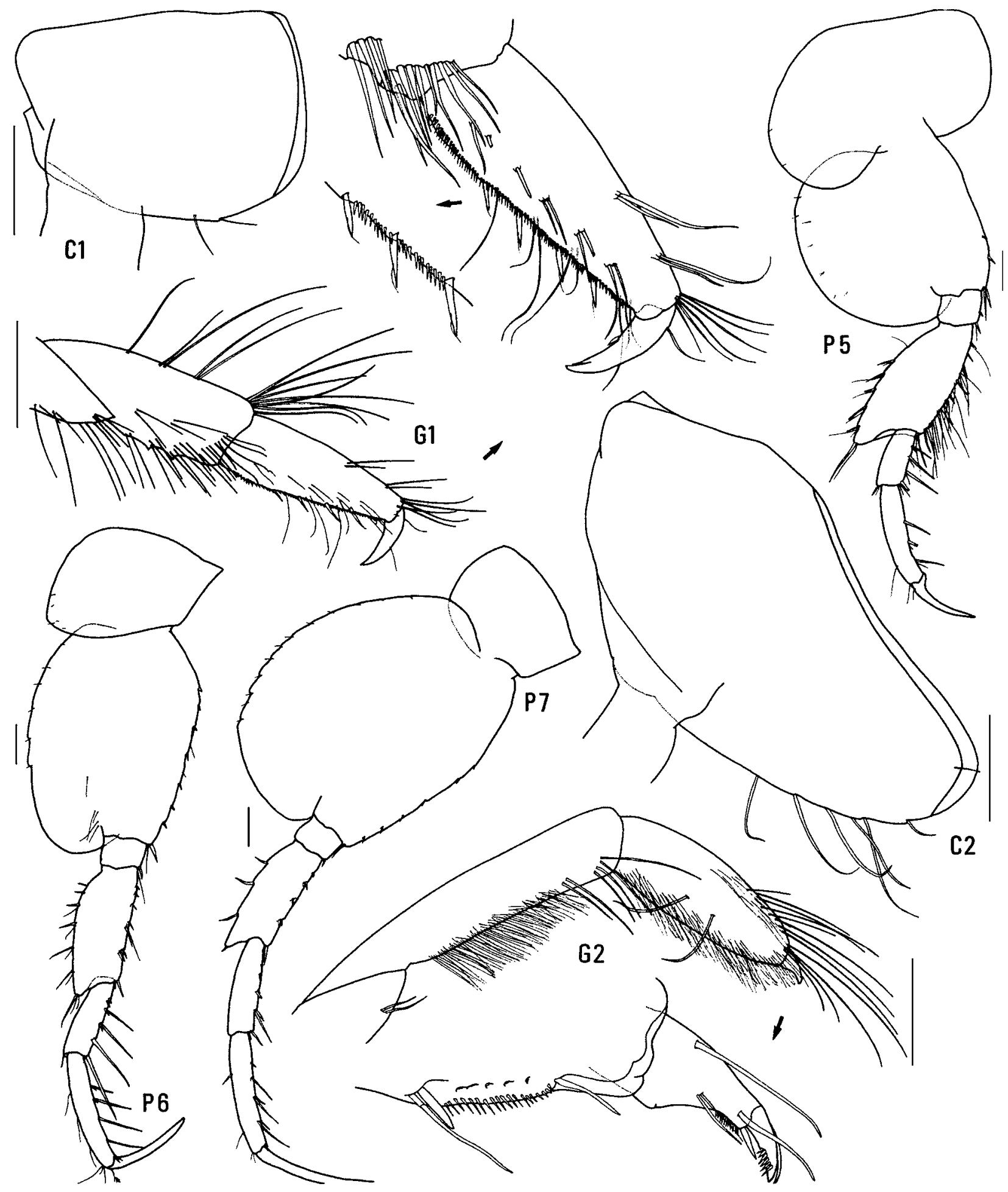

Figure 75. Devo grahami n.sp., holotype female, 7.7 mm, AM P36872, east of Broken Bay, NSW. Scales represent 0.2 mm.

with setal fringe. Uropod 3 rami with plumose setae.

Etymology. Named for Ken Graham, fisheries biologist on the FRV Kapala, who helped immensely over the years in collecting amphipods and other invertebrates during the cruises of the Kapala.

Remarks. Devo grahami is very similar to D. dubuc but is distinguished by the notch on the anterior margin of the head not extended into a slit, and by plumose setae present on uropod 3 of the female.

Habitat. Devo grahami has been collected on fine and coarse sediment bottoms.

Distribution. Southeastern Australia, Tasman Sea; 549$1840 \mathrm{~m}$ depth. 


\section{Pseudamaryllis Andres}

Pseudamaryllis Andres, 1981: 464.-Barnard \& Karaman, 1991: 521.-Lowry \& Stoddart, 1993: 98.

Amaryllis.-Ledoyer, 1986: 717 (in part).

Paravijaya Ren, 1998: 156, 162.

Diagnosis. Head with rostrum anteriorly rounded or absent; eye reniform. Antenna 1 peduncular article 1 not ball-shaped proximally; peduncular article 2 medium length; callynophore present in female and male. Antenna 2 flagellum about as long as that of antenna 1 in female and male. Mandible palp article 3 with or without proximal A3-seta. Pereopod 4 coxa with anterior and posterior margins subparallel. Uropod 3 rami without plumose setae in male and female; outer ramus 1-articulate.

Type species. Pseudamaryllis nonconstricta Andres, 1981, by original designation.

Species composition. Pseudamaryllis contains two species: P. andresi Lowry \& Stoddart, 1993 and P. nonconstricta Andres, 1981.

Remarks. Ren (1998) established the genus Paravijaya, which he compared to and distinguished from the genera Amaryllis, Pseudamaryllis and Vijaya. Paravijaya was distinguished from Pseudamaryllis by: the labrum and epistome being coalesced, peduncular article 1 of antenna 1 being longer than other articles, and inner ramus of uropod 2 with a small notch. Ren was apparently unaware of the publication by Lowry \& Stoddart (1993), which established a new species of Pseudamaryllis and slightly expanded the generic diagnosis. Paravijaya is a synonym of Pseudamaryllis and $\mathrm{Pa}$. apiculata Ren, 1998, is the same species described by Lowry \& Stoddart (1993) as Ps. andresi.

Pseudamaryllis is the only amaryllidid genus with the combination of a callynophore in the female and robust setae on the propodus of gnathopod 1.

Distribution. Western Indian Ocean, Red Sea and South China Sea; 90-1544 m depth (see Fig. 76).

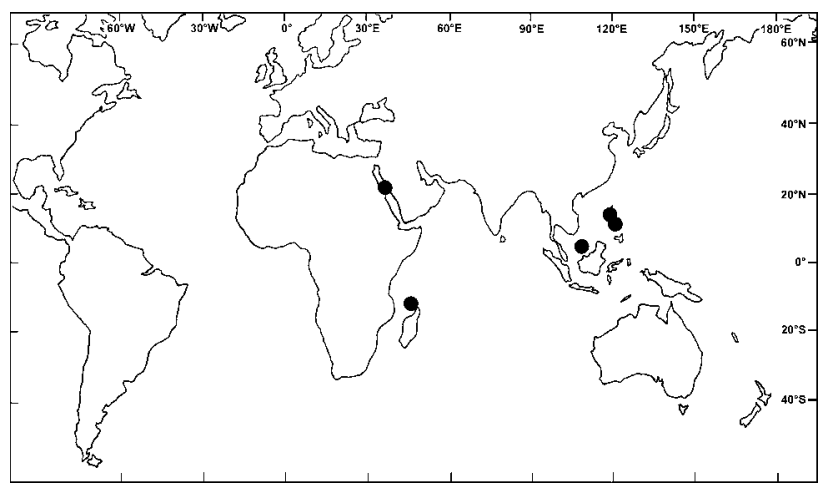

Figure 76. Distribution of genus Pseudamaryllis.

\section{Pseudamaryllis andresi Lowry \& Stoddart}

Pseudamaryllis andresi Lowry \& Stoddart, 1993: 99, figs. 2830.-Springthorpe \& Lowry, 1994: 8.

Paravijaya apiculata Ren, 1998: 157, 162, figs. 1, 2.

Distribution. Spratly Islands and Philippines, South China Sea; 90-127 m depth.

\section{Vijaya Walker}

Vijaya Walker, 1904: 241.-Stebbing, 1906: 717.-Gurjanova, 1962: 45.-J.L. Barnard, 1964: 63.-J.L. Barnard, 1969: 368.-Barnard \& Karaman, 1991: 541.-Lowry \& Stoddart, 2002.

Diagnosis. Head with rostrum anteriorly rounded; eye ventrally tapered. Antenna 1 peduncular article 1 not ballshaped proximally; peduncular article 2 medium length; callynophore absent in female, present in male. Antenna 2 flagellum about as long as that of antenna 1 in female, longer than body in male. Mandible palp article 3 with proximal A3-seta in male, without proximal A3-seta in female. Pereopod 4 coxa with anterior and posterior margins subparallel. Uropod 3 rami with plumose setae in male and female; outer ramus 1-articulate.

Type species. Vijaya tenuipes Walker, 1904, by monotypy.

Species composition. Vijaya contains one species: V. tenuipes Walker, 1904.

Remarks. When Pirlot (1933) described Bathyamaryllis, the genus Vijaya was so poorly known that he could not have realised the close relationship of his species to the species that Walker (1904) described. We (Lowry \& Stoddart, 2002) have examined Walker's material and redescribed the species based on new material from the Andaman Sea. This has shown several generic level differences between these species. Vijaya has a ventrally tapered eye and a unique acutely produced anteroventral corner on coxa 4 ; it does not have the proximally ball-shaped peduncular article 1 of antenna 1 that characterizes Bathyamaryllis and Devo. Pseudamaryllis has reniform eyes, a callynophore in the female and subparallel anterior and posterior margins on coxa 4.

Vijayiines typically live in the deep sea; Vijaya is the only member of the group that lives in shallow tropical waters.

The female of Vijaya tenuipes has oostegites on gnathopod 1 to pereopod 5; it is the only amaryllidid species known to have an oostegite on gnathopod 1 . The only other lysianassoid we know to have such an array of oostegites is Eurythenes gryllus.

Distribution. Northern Indian Ocean and Andaman Sea; shallow water to $68 \mathrm{~m}$ depth (see Fig. 77).

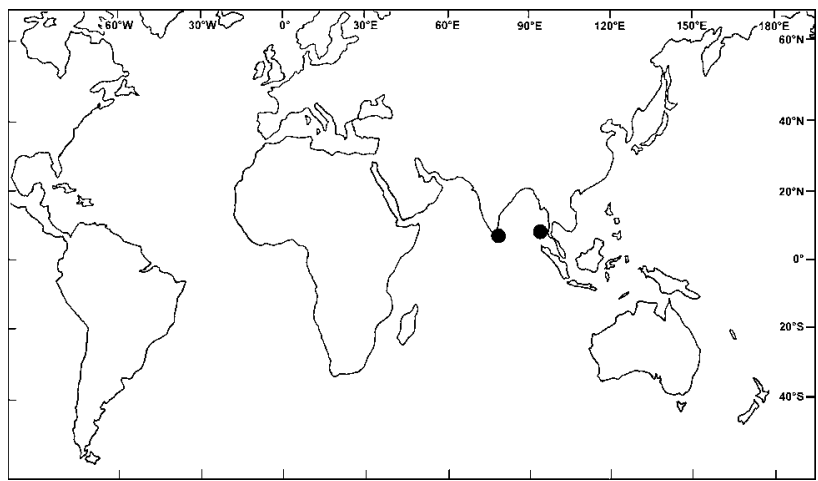

Figure 77. Distribution of genus Vijaya. 

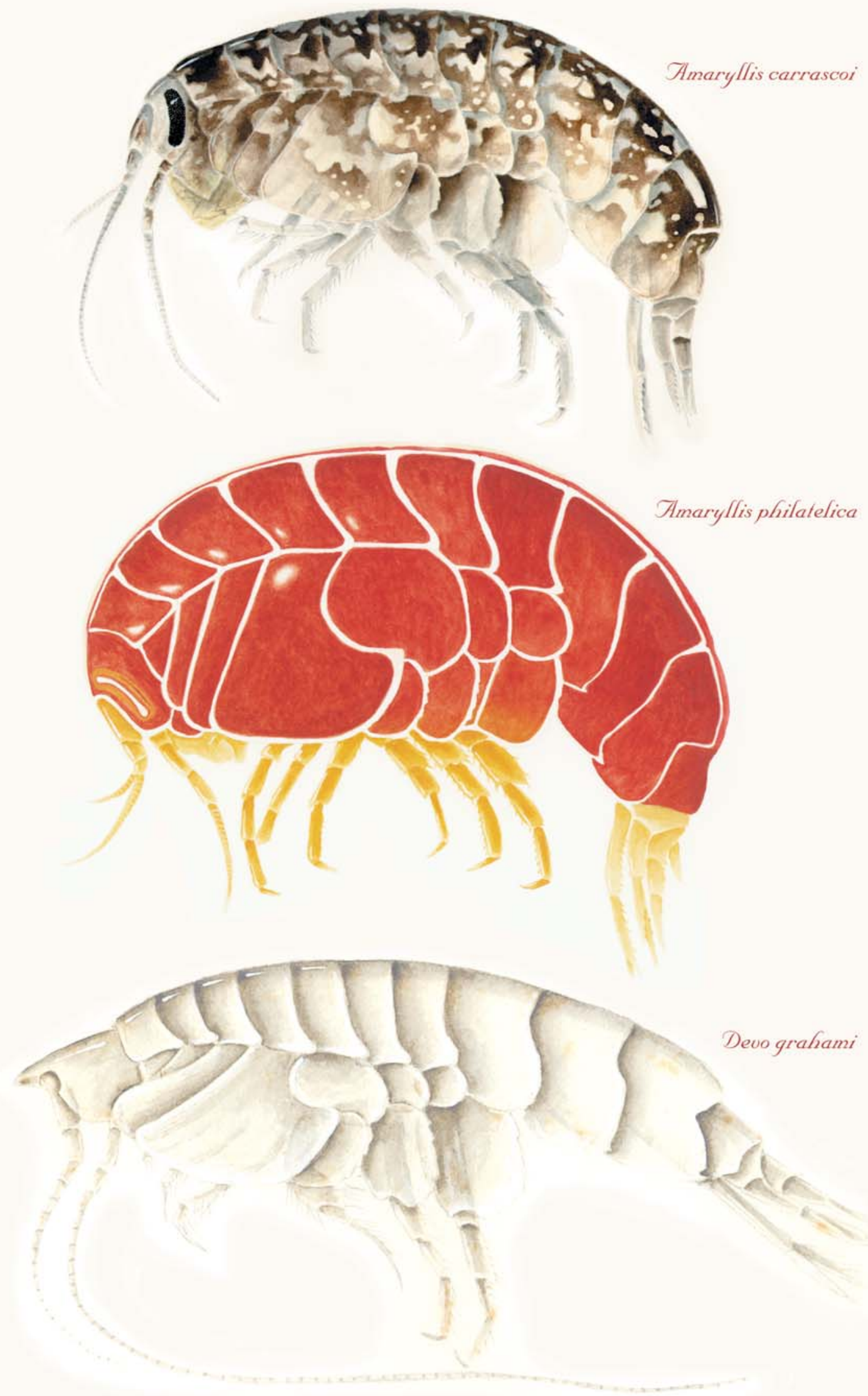

Plate 1. Watercolours by Sharne Wiedland, from photographs of living specimens, showing the diversity of colour in amaryllidids. These figures show the camouflage pattern of Amaryllis carrascoi $\mathrm{n}$.sp. ( $a$, upper), the warning colours of Amaryllis philatelica n.sp. (b, middle) and the lack of colour in the deep-sea species Devo grahami n.sp. (c, lower) 
ACKNOWLEDGMENTS. We thank Gary Poore (Museum of Victoria) and Wolfgang Zeidler (South Australian Museum) for the loan of large amounts of material; Ken Graham (NSW State Fisheries) for material collected by the FRV Kapala; Stephen Keable, Kate Dempsey and Rachael Peart for illustrations; and Roger Springthorpe for making the plates. Parts of this project were supported by grants from the Australian Research Council and the Australian Biological Resources Study.

\section{References}

Alonso, G.M., 1987. Estudios sistematicos de tres Lysianassidae (Amphipoda, Gammaridea) de la Argentina. Physis Seccion A: Oceanos y sus Organismos 45(108): 1-10.

Andres, H.G., 1981. Lysianassidae aus dem Abyssal des Roten Meeres. Bearbeitung der Köderfänge von FS "Sonne"MESEDA I (1977) (Crustacea: Amphipoda: Gammaridea). Senckenbergiana Biologica 61(5/6): 429-443.

Barnard, J.L., 1958. Index to the families, genera, and species of the gammaridean Amphipoda (Crustacea). Allan Hancock Foundation Publications, Occasional Paper 19: 1-145.

Barnard, J.L., 1964. Revision of some families, genera and species of gammaridean Amphipoda. Crustaceana 7(1): 49-74.

Barnard, J.L., 1969. The families and genera of marine gammaridean Amphipoda. United States National Museum Bulletin 271: 1-535.

Barnard, J.L., 1972. Gammaridean Amphipoda of Australia, Part I. Smithsonian Contributions to Zoology 103: 1-333.

Barnard, J.L., 1974. Gammaridean Amphipoda of Australia, Part II. Smithsonian Contributions to Zoology 139: 1-148.

Barnard, J.L., \& G.S. Karaman, 1991. The families and genera of marine gammaridean Amphipoda (except marine gammaroids). Records of the Australian Museum, Supplement 13(2): 419-866.

Barnard, K.H., 1916. Contributions to the crustacean fauna of South Africa. 5-The Amphipoda. Annals of the South African Museum 15: 105-302, pls 26-28.

Barnard, K.H., 1925. Contributions to the crustacean fauna of South Africa. No. 8. Further additions to the list of Amphipoda. Annals of the South African Museum 20: 319-380, pl. 34.

Barnard, K.H., 1932. Amphipoda. Discovery Reports 5: 1-326, pl. 1.

Barnard, K.H., 1937. Amphipoda. Scientific Reports of the John Murray Expedition 4(6): 131-201.

Barnard, K.H., 1940. Contributions to the crustacean fauna of South Africa. 12. Further additions to the Tanaidacea, Isopoda, and Amphipoda, together with keys for the identification of the hitherto recorded marine and fresh-water species. Annals of the South African Museum 32: 381-543.

Bonnier, J., 1896. Résultats Scientifiques de la Campagne du «Caudan» dans la Golfe de Gascogne-Août-Septembre 1895. Edriophthalmes. Annales de l'Universite de Lyon 26: 527-689, pls. 28-40.

Chevreux, E., 1903. Campagnes Scientifique de S.A. le Prince Albert 1er de Monaco. Note préliminaire sur les amphipodes de la famille des Lysianassidae recueillis par la Princesse-Alice dans les eaux profondes de l'Atlantique et de la Méditerranée. Bulletin de la Société Zoologique de France 28: 81-97.

Chevreux, E., 1911. Diagnoses d'amphipodes nouveaux provenant des campagnes de la Princesse-Alice dans l'Atlantique nord (suite). Bulletin de l'Institut Océanographique, Monaco 204: 1-13.

Chevreux, E., 1935. Amphipodes provenant des campagnes du Prince Albert 1er de Monaco. Résultats des Campagnes Scientifiques Accomplies sur son Yacht par Albert Ier Prince Souverain de Monaco 90: 1-214, pls 1-16.

Chilton, C., 1885. Notes on a few Australian Edriophthalmata. Proceedings of the Linnean Society of New South Wales 9(4): 1035-1044, pls 46, 47.
Chilton, C., 1906. Report of some Crustacea dredged off the coast of Auckland. Transactions of the New Zealand Institute 38: 265-268.

Chilton, C., 1912. The Amphipoda of the Scottish National Antarctic Expedition. Transactions of the Royal Society of Edinburgh 48: 455-520, pls 1, 2.

Chilton, C., 1921. Report on the Amphipoda obtained by the F.I.S. "Endeavour" in Australian seas. Biological Results of the Fishing Experiments carried on by the F.I.S. "Endeavour", 1909-14 5(2): 33-92.

Dallwitz, M.J., T.A. Paine \& E.J. Zurcher, 1993 onwards. User's Guide to the DELTA System: A General System for Processing Taxonomic Descriptions. 4th edition. http://biodiversity.uno.edu/delta/

Dallwitz, M.J., T.A. Paine \& E.J. Zurcher, 1998. Interactive keys. In Information Technology, Plant Pathology and Biodiversity, ed. P. Bridge, P. Jeffries, D.R. Morse and P.R. Scott, pp. 201212. Wallingford: CAB International.

Day, J.H., J.G. Field \& M.J. Penrith, 1970. The benthic fauna and fishes of False Bay, South Africa. Transactions of the Royal Society of South Africa 39(1): 1-108.

Debelius, H., 1999. Crustacea Guide of the World. (English Edition). Frankfurt: IKAN, $321 \mathrm{pp}$.

Della Valle, A., 1893. Gammarini del Golfo di Napoli. Fauna und Flora des Golfes von Neapel 20: 1-948, pls. 1-61.

Edgar, G.J., 1997. Australian Marine Life-The Plants and Animals of Temperate Waters. Kew: Reed Books, 544 pp.

Gonzalez, E., 1991. Actual state of gammaridean amphipoda taxonomy and catalogue of species from Chile. Hydrobiologia 223: 47-68.

Griffiths, C.L., 1973. The Amphipoda of southern Africa. Part 1. The Gammaridea and Caprellidea of southern Moçambique. Annals of the South African Museum 60(10): 265-306.

Griffiths, C.L., 1974a. The Amphipoda of southern Africa. Part 2. The Gammaridea and Caprellidea of South West Africa south of $20^{\circ} \mathrm{S}$. Annals of the South African Museum 62(6): 169-208.

Griffiths, C.L., 1974b. The Amphipoda of southern Africa. Part 3. The Gammaridea and Caprellidea of Natal. Annals of the South African Museum 62(7): 209-264.

Griffiths, C.L., 1974c. The Amphipoda of southern Africa. Part 4. The Gammaridea and Caprellidea of the Cape Province east of Cape Agulhas. Annals of the South African Museum 65(9): 251336.

Griffiths, C.L., 1975. The Amphipoda of southern Africa. Part 5. The Gammaridea and Caprellidea of the Cape Province west of Cape Agulhas. Annals of the South African Museum 67(5): 91-181.

Griffiths, C.L., 1976. Guide to the Benthic Marine Amphipods of Southern Africa. Cape Town: Trustees, South African Museum, $106 \mathrm{pp}$.

Griffiths, C.L., 1977. The South African Museum's Meiring Naude cruises. Part 6. Amphipoda. Annals of the South African Museum 74(4): 105-123.

Grindley, J.R., \& B.F. Kensley, 1966. Benthonic marine fauna obtained off the Orange River mouth by the diamond dredger Emerson-K. Cimbebasia 16: 1-14.

Guiler, E.R., 1952. A list of the Crustacea of Tasmania. Records of the Queen Victoria Museum, Launceston 3(3): 15-44.

Gurjanova, E., 1962. [Amphipods of the northern part of the Pacific Ocean (Amphipoda-Gammaridea) Part 1]. Opredeliteli po Faune SSSR Akademiya Nauk SSSR 74: 1-440 (in Russian).

Hale, H.M., 1929. The Crustaceans of South Australia. Part II. Pp. 201-381. Adelaide: H. Weir, Government Printer.

Haswell, W.A., 1879. On Australian Amphipoda. Proceedings of the Linnean Society of New South Wales 4(3): 245-279, pls. 7-12.

Haswell, W.A., 1880. Preliminary report on the Australian Amphipoda. Annals and Magazine of Natural History, Series 5, 5: 30-34.

Haswell, W.A., 1882. Catalogue of the Australian Stalk- and Sessileeyed Crustacea. Sydney: Australian Museum, 324 pp, 4 pls. 
Hutchings, P., J.T. Van Der Velde \& S.J. Keable, 1989. Baseline survey of the benthic macrofauna of Twofold Bay, N.S.W., with a discussion of the marine species introduced into the Bay. Proceedings of the Linnean Society of New South Wales 110(4): 339-367.

Hutton, F.W., 1904. Index Faunae Novae Zealandiae. London: P. Dulau and Co., 372 pp.

Karacsonyi, T., 1997. Brilliant fleas of the sea. Australian Geographic 45: 29.

Kensley, B.F., \& M.J. Penrith, 1977. Biological survey of Sandris. 1. Introduction and faunal list. Madoqua 10(3): 181-190.

Lawson, G.S., P.A. Tyler \& C.M. Young, 1993. Attraction of deepsea amphipods to macrophyte food falls. Journal of Experimental Marine Biology and Ecology 169: 33-39.

Ledoyer, M., 1978. Amphipodes gammariens (Crustacea) des biotopes cavitaires organogènes récifaux de l'île Maurice (Océan Indien). The Mauritius Institute Bulletin 8(3): 197-332.

Ledoyer, M., 1979. Les gammariens de la pente externe du Grand Récif de Tuléar (Madagascar) (Crustacea Amphipoda). Memorie del Museo Civico di Storia Naturale, Verona. 2nd Serie, Sezione Scienze della Vita 2: 1-150.

Ledoyer, M., 1986. Crustacés Amphipodes Gammariens. Familles des Haustoriidae à Vitjazianidae. Faune de Madagascar 59(2): 599-1112.

Lowry, J.K., \& S. Bullock, 1976. Catalogue of the marine gammaridean Amphipoda of the Southern Ocean. The Royal Society of New Zealand Bulletin 16: 1-187.

Lowry, J.K., \& H.E. Stoddart, 1987. A new South American genus and species in the amaryllidid group of lysianassoid Amphipoda. Journal of Natural History 21: 1303-1309.

Lowry, J.K., \& H.E. Stoddart, 1993. Crustacea Amphipoda: Lysianassoids from Philippine and Indonesian waters. In Résultats des Campagnes MUSORSTOM, ed. A. Crosnier, volume 10. Mémoires du Muséum National d'Histoire Naturelle, Paris 156: 55-109.

Lowry, J.K., \& H.E. Stoddart, 1994. Crustacea Amphipoda: Lysianassoids from the tropical western South Pacific Ocean. In Résultats des Campagnes MUSORSTOM, ed. A. Crosnier, volume 12. Mémoires du Muséum National d'Histoire Naturelle, Paris 161: 127-223.

Lowry, J.K., \& H.E. Stoddart, 2002. First records of lysianassoid amphipods (Crustacea) from the Andaman Sea. In Biodiversity of Crustacea of the Andaman Sea. Proceedings of the International Workshop on the Crustacea in the Andaman Sea, Phuket Marine Biological Center, 29 November-20 December 1998, ed. N.L. Bruce, M. Berggren \& S. Bussawarit. Phuket Marine Biological Center Special Publication 23(1): 165-188.

Lyons, J., \& A.A. Myers, 1991. Amphipoda Gammaridea from coral rubble in the Gulf of Aqaba, Red Sea: families Dexaminidae, Eusiridae, Isaeidae, Ischyroceridae, Leucothoidae, Liljeborgiidae and Lysianassidae. Journal of Natural History 25: 597-621.

Pirlot, J.M., 1933. Les amphipodes de l'expédition du Siboga. Deuxième partie: Les amphipodes gammarides, II:-Les amphipodes de la mer profonde. 1 (Lysianassidae, Stegocephalidae, Stenothoidae, Pleustidae, Lepechinellidae). Siboga-Expeditie, Monograph 33c: 114-167.

Pirlot, J.M., 1939. Amphipoda. Résultats scientifiques des croisières du navire-école Belge «Mercator». Mémoires du Musée Royal d'Histoire Naturelle de Belgique, ser. 2, 15: 47-80.

Poore, A.G.B. \& J.K. Lowry, 1997. New ampithoid amphipods from Port Jackson, New South Wales, Australia (Crustacea: Amphipoda: Ampithoidae). Invertebrate Taxonomy 11: 897-941.

Poore, G.C.B., S.F. Rainer, R.B. Spies \& E. Ward, 1975. The zoobenthos program in Port Phillip Bay, 1969-73. Fisheries and Wildlife Paper, Victoria 7: 1-78.
Ren, X., 1998. [A new genus and two new species of the family Lysianassidae (Crustacea: Amphipoda) from Nansha Islands, South China Sea]. Studies on Marine Fauna and Flora of the Nansha Islands and Neighbouring Waters 3: 156-165. (in Chinese and English)

Schellenberg, A., 1926. Amphipoda 3: Die Gammariden der Deutschen Tiefsee-Expedition. Wissenschaftliche Ergebnisse der Deutschen Tiefsee-Expedition auf dem Dampfer "Valdivia" 1898-1899 23(5): 193-243, pl. 5.

Schellenberg, A., 1931. Gammariden und Caprelliden des Magellangebietes, Südgeorgiens und der Westantarktis. Further Zoological Results of the Swedish Antarctic Expedition 19011903 2(6): 1-290, pl. 1.

Sergeev, V.N., S.M. Clarke \& S.A. Shepherd, 1988. Motile macroepifauna of the seagrasses, Amphibolis and Posidonia, and unvegetated sandy substrata in Holdfast Bay, South Australia. Transactions of the Royal Society of South Australia 112: 97-108.

Sheard, K., 1937. A catalogue of Australian Gammaridea. Transactions of the Royal Society of South Australia 61: 17-29.

Springthorpe, R.T., \& J.K. Lowry, 1994. Catalogue of crustacean type specimens in the Australian Museum: Malacostraca. Technical Reports of the Australian Museum 11: 1-134.

Stebbing, T.R.R., 1888. Report on the Amphipoda collected by H.M.S. Challenger during the years 1873-1876. Report on the Scientific Results of the Voyage of H.M.S. Challenger during the years 1873-76, Zoology, 29: 1-1737, pls. 1-210.

Stebbing, T.R.R., 1906. Amphipoda. I. Gammaridea. Das Tierreich 21: $1-806$.

Stebbing, T.R.R., 1908. South African Crustacea. (Part IV). Annals of the South African Museum 6: 1-96, pls. 1-15.

Stebbing, T.R.R., 1910a. Scientific results of the trawling expedition of H.M.C.S. "Thetis". Crustacea. Part V. Amphipoda. Memoirs of the Australian Museum 4: 565-658, pls. 47-60.

Stebbing, T.R.R., 1910b. General catalogue of South African Crustacea (Part V. of S.A. Crustacea, for the Marine Investigations in South Africa). Annals of the South African Museum 6: 281-593, pls. 15-22.

Stephensen, K., 1923. Crustacea Malacostraca, V: (Amphipoda, I). Danish Ingolf-Expedition 3(8): 1-100.

Thomson, G.M., 1902. Some recent additions to and notes on the crustacean fauna of New Zealand. Annals and Magazine of Natural History, Series 7, 10: 462-465.

Thurston, M.H., \& E. Allen, 1969. Type material of the families Lysianassidae, Stegocephalidae, Ampeliscidae and Haustoriidae (Crustacea: Amphipoda) in the collections of the British Museum (Natural History). Bulletin of the British Museum (Natural History) (Zoology) 17: 347-388.

Walker, A.O., 1904. Report on the Amphipoda collected by Professor Herdman, at Ceylon, in 1902. Ceylon Pearl Oyster Fisheries1904. Supplementary Reports 17: 229-300, pls. 1-8.

Walker, A.O., 1909. Amphipoda Gammaridea from the Indian Ocean, British East Africa, and the Red Sea. Transactions of the Linnean Society of London, Series 2, Zoology 12(4): 323344 , pls. $42-43$.

Whitelegge, T., 1889. List of the marine and fresh-water invertebrate fauna of Port Jackson and the neighbourhood. Journal and Proceedings of the Royal Society of New South Wales 23: 163-323.

Manuscript received 8 November 2000, revised 31 July 2001 and accepted 31 August 2001.

Associate Editor: G.D.F. Wilson. 
Index

Amaryllididae ................................. 131

Amaryllidinae ................................ 132

Amaryllis ......................................... 132

andresi, Pseudamaryllis .................. 210

anomala, Bamarooka ...................... 176

Bamarooka ................................... 175

Bathyamaryllis .................................. 199

bathycephala, Bamarooka ................ 179

brevicornis, Amaryllis....................... 134

carrascoi, Amaryllis ....................... 137

croca, Amaryllis ............................... 143

Devo .................................................... 201

dianae, Amaryllis ............................. 143

dinjerra, Bamarooka ....................... 182

dubuc, Devo .................................. 204

endota, Bamarooka.......................... 185

Erikus ................................................. 195

grahami, Devo ................................ 204 kamata, Amaryllis ........................... 147

kapala, Bathyamaryllis .................... 200

keablei, Amaryllis ............................ 150

kimbla, Bamarooka ........................... 189

macrophthalma, Amaryllis ............... 152

migo, Amaryllis ............................... 157

moona, Amaryllis ............................. 160

olinda, Amaryllis ................................ 163

philatelica, Amaryllis ...................... 166

Pseudamaryllis ............................... 210

quokka, Amaryllis ............................ 169

spencerensis, Amaryllis .................... 173

tropicalis, Bamarooka ..................... 192

Vijaya ............................................. 210

Vijayiinae .......................................... 199

wonga, Wonga ................................. 196

Wonga .............................................. 195 